\title{
Hydrosulfonylation of Unactivated Alkenes by Visible Light
}

\section{Photoredox Catalysis}

\author{
Juan-Juan Wang and Wei Yu*
}

State Key Laboratory of Applied Organic Chemistry, College of Chemistry and

Chemical Engineering, Lanzhou University

Lanzhou, Gansu 730000, China

Fax: +86-931-8912582; Email: yuwei@lzu.edu.cn

\section{Supporting Information}

\section{Table of Contents}

General information

Experimental procedures

Optimazation of reaction conditions S5

Deuterium experiment

Quantum yield measurement

Characterization data

References

${ }^{1} \mathrm{H}$ and ${ }^{13} \mathrm{C}$ NMR spectra 


\section{General information}

The ${ }^{1} \mathrm{H}$ and ${ }^{13} \mathrm{C}$ NMR spectra were recorded on a Bruker AVANCE III-400 MHz spectrometer or a Bruker INOVA- $600 \mathrm{MHz}$ spectrometer with $\mathrm{CDCl}_{3}$ or DMSO- $d 6$ as the solvent. In $\mathrm{CDCl}_{3}$, the chemical shifts in ${ }^{1} \mathrm{H}$ NMR spectra were determined with $\mathrm{Si}\left(\mathrm{CH}_{3}\right)_{4}$ as the internal standard $(\delta=0.00 \mathrm{ppm})$; the chemical shifts in ${ }^{13} \mathrm{C} \mathrm{NMR}$ spectra were determined based on the chemical shift of $\mathrm{CDCl}_{3}(\delta=77.00 \mathrm{ppm})$. In DMSO- $d_{6}$, the chemical shifts in ${ }^{1} \mathrm{H}$ NMR and ${ }^{13} \mathrm{C}$ NMR spectra were determined based on the chemical shift of DMSO- $d_{6}(\delta=2.50 \mathrm{ppm}$ and $\delta=39.52 \mathrm{ppm}$, respectively). The coupling constant (s) ( $J$ value) are reported in $\mathrm{Hz}$ ( $\mathrm{s}=$ singlet, $\mathrm{d}=$ doublet, $\mathrm{t}=$ triplet, $\mathrm{q}=$ quadruplet, $\mathrm{m}=$ multiplet or unresolved, $\mathrm{br}=$ broad signal). The high resolution mass spectra (HRMS) were measured on a Bruker micrOTOF QII by ESI. The Fourier transformation infrared spectra (FT-IR) were measured on a NEXUS 670 spectrometer. Melting points were measured on an XT-4 melting point apparatus and were uncorrected. A UV-visible spectrophotometer with a $\mathrm{D}_{2}$ lamp was used for the quantum yield measurement. Flash column chromatography (FCC) was carried out on silica gel (200-300 mesh). The photochemical reactions were conducted with a blue LED irradiating system provided by Shenzhen Chunda Xiguang Photoelectronics Co. Ltd. of China (Model GHX-4). Dichloromethane (DCM) was distilled from $\mathrm{P}_{2} \mathrm{O}_{5}$ before use. Other solvents were treated before use following the standard procedures. $\left.\left[\operatorname{Ir}\left(\mathrm{dF}_{(\mathrm{CF}}\right) \mathrm{ppy}\right)_{2}(\mathrm{dtbbpy})\right] \mathrm{PF}_{6}$ was prepared according to the reported procedure. ${ }^{1}$ The unsaturated amides were synthesized according to the previously described procedures. ${ }^{2,3}$ All the other materials were purchased from Sigma-Aldrich, Alfa Aesar, Acros, TCI, Adamas, Energy Chemical, Aladdin or Ark Pharm, and were used without further purification.

\section{Experimental procedures}

General procedure for the preparation of unsaturated carbamates ${ }^{2}$

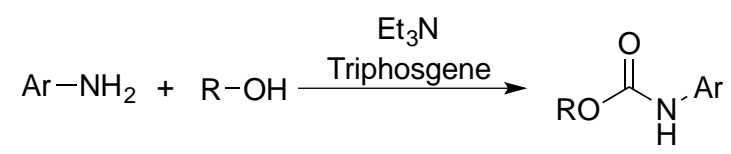

A flame-dried $250 \mathrm{~mL}$ round-bottomed flask was degassed, flushed with nitrogen, and charged with triphosgene $(1.49 \mathrm{~g}, 5.0 \mathrm{mmol})$ in THF $(10 \mathrm{~mL})$. A solution of amine $(0.465 \mathrm{~g}, 5.0 \mathrm{mmol})$ dissolved in THF (40 mL) was slowly dripped into the triphosgene solution, followed by slow addition of $1.5 \mathrm{~mL}$ of triethylamine $(1.5 \mathrm{~mL}$, $10.5 \mathrm{mmol})$. The mixture was stirred at room temperature for $2 \mathrm{~h}$. The reaction mixture was then concentrated on a rotary evaporator, and the flask containing the residual was degassed, and then charged with acetonitrile $(80 \mathrm{~mL})$, triethylamine $(1.5$ $\mathrm{mL}, 10.5 \mathrm{mmol})$ and the unsaturated alcohol $(6.0 \mathrm{mmol})$. The reaction mixture was 
stirred at $70{ }^{\circ} \mathrm{C}$ for $8 \mathrm{~h}$. After that, the mixture was concentrated on a rotary evaporator, and the residual was purified by alumina column chromatography (gradient elution from petroleum ether $(\mathrm{PE})$ to ethyl acetate $(\mathrm{EA})(\mathrm{EA} / \mathrm{PE}=1: 4, \mathrm{v} / \mathrm{v})$ to yield the carbamate product.

\section{General procedure for the preparation of the tertiary amides 3}

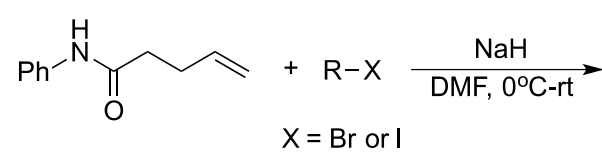<smiles>[R]N(C(=O)CCC=C)c1ccccc1</smiles>

A solution of the prepared secondary amide $(3.1 \mathrm{mmol})$ in dry DMF $(13 \mathrm{~mL})$ was added into a suspension of $\mathrm{NaH}(60 \%$ dispersion in mineral oil, $0.15 \mathrm{~g}, 3.73 \mathrm{mmol})$ in dry DMF (4 mL) at $0{ }^{\circ} \mathrm{C}$ (in an ice-water bath). After stirring the resulting solution for $15 \mathrm{~min}$, iodoethane, benzyl bromide or allyl bromide $(9.3 \mathrm{mmol})$ was added dropwise into it. The ice bath was then removed, and the reaction mixture was stirred at room temperature until TLC analysis indicated that the starting material was no longer present (3-4 h). The DMF was removed under reduced pressure, and the resulting yellow residual was treated with water $(20 \mathrm{~mL})$ and extracted with ethyl acetate $(3 \times 20$ $\mathrm{mL}$ ). The combined organic layers were dried over anhydrous sodium sulfate, and the volatile components were removed under reduced pressure. The residual was treated with silica gel column chromatography (PE and EA) to give the pure product.

\section{General procedure for the preparation of sodium aryl sulfinates ${ }^{4}$}

Sodium sulfite ( $2.50 \mathrm{~g}, 0.02 \mathrm{~mol}, 2.0$ equiv), sodium bicarbonate $(1.68 \mathrm{~g}, 0.02 \mathrm{~mol}$, 2.0 equiv) and the corresponding aryl sulfonyl chloride $(0.01 \mathrm{~mol})$ were dissolved in distilled water $(9.60 \mathrm{~mL})$. The reaction mixture was stirred at $80{ }^{\circ} \mathrm{C}$ for $4 \mathrm{~h}$. After cooling to room temperature, the mixture was washed with ethyl acetate, the aqueous layer was removed under reduced pressure at $60{ }^{\circ} \mathrm{C}$, the white solid was washed with ethanol $(3 \times 100 \mathrm{~mL})$. The combined organic layers were dried over anhydrous sodium sulfate, and ethanol was removed under reduced pressure to give the desired sodium aryl sulfinates as white crystalline powder.

\section{General procedure for the preparation of but-3-en-1-yl 1-methyl-1H-pyrrole-2-} carboxylate $^{5}$

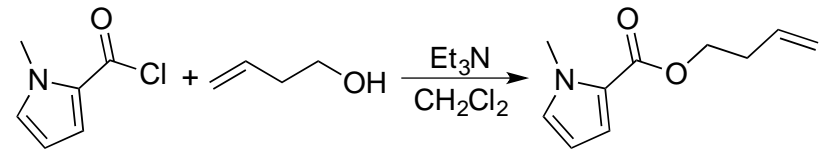

In a $100 \mathrm{~mL}$ flask equipped with a magnetic stirring bar, triethylamine $(5.0 \mathrm{~mL}$, $36.0 \mathrm{mmol}, 3.6$ equiv) was added to a solution of but-3-en-1-ol $(0.72 \mathrm{~g}, 10.0 \mathrm{mmol})$ in $40 \mathrm{~mL}$ dichloromethane. 1-Methyl-1H-pyrrole-2-carbonyl chloride (1.72 g, 12.0 mmol, 1.2 equiv) was added slowly using a syringe. The solution became cloudy and the reaction flask became warm to the touch. The reaction was stirred at room temperature under argon for $18 \mathrm{~h}$, after which it was quenched with saturated aqueous 
ammonium chloride and extracted with dichloromethane. The combined organic phases were washed with brine, dried with anhydrous sodium sulfate, and concentrated under reduced pressure. The residual was purified via column chromatography with PE and EA (v/v = 4:1) as the eluent to afford the title compound as a colorless liquid.

\section{General procedure for hydrosulfonylation of alkenes}

$$
\mathrm{R}^{1} \curvearrowright+\mathrm{R}^{2} \mathrm{SO}_{2} \mathrm{Na} \frac{\stackrel{\left[\mathrm{Ir}\left(\mathrm{dF}\left(\mathrm{CF}_{3}\right) \mathrm{ppy}\right)_{2}(\mathrm{dtbbpy})\right] \mathrm{PF}_{6}(1 \mathrm{~mol} \%)}{\mathrm{H}_{2} \mathrm{O}(10 \text { equiv), AcOH }(4.5 \text { equiv })}}{\mathrm{CH}_{2} \mathrm{Cl}_{2}(0.2 \mathrm{M}), \text { blue LEDs, } \mathrm{N}_{2}, \mathrm{rt}} \mathrm{R}^{1} \mathrm{SO}_{2} \mathrm{R}^{2}
$$

\section{Scheme S1}

A flame-dried glass tube $(20 \mathrm{~mL})$ equipped with a magnetic stirring bar and a rubber stopper was charged with the starting material $(0.5 \mathrm{mmol}), \mathrm{R}^{2} \mathrm{SO}_{2} \mathrm{Na}(0.825$

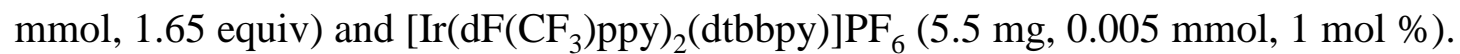
The tube was evacuated and backfilled with nitrogen (three times), and into which was added $2.5 \mathrm{~mL}$ of $\mathrm{CH}_{2} \mathrm{Cl}_{2}$ and $90 \mu \mathrm{L}$ of $\mathrm{H}_{2} \mathrm{O}$, followed by $\mathrm{AcOH}(0.135 \mathrm{~g}, 2.25$ $\mathrm{mmol})$. The tube was put at a distance of $2 \mathrm{~cm}$ from blue LED lamps (6 W, equipped with a cooling fan) and progress of the reaction was monitored periodically by TLC. Upon completion, the reaction was quenched with saturated $\mathrm{NaHCO}_{3}(3 \mathrm{~mL})$, and the aqueous phase was extracted with $\mathrm{CH}_{2} \mathrm{Cl}_{2}(3 \times 5 \mathrm{~mL})$. The combined organic phases were dried over anhydrous $\mathrm{Na}_{2} \mathrm{SO}_{4}$, and then concentrated under reduced pressure on a rotary evaporator. The residual was subjected to silica gel column chromatography (eluent: PE and EA) to afford the product.

The LED lamps consists of six beads $(6 \times 1 \mathrm{~W})$. The parameters of LED light are as follows: Peak wavelength: $456.2 \mathrm{~nm}$; main wavelength: $460.6 \mathrm{~nm}$; half-wave width: $21 \mathrm{~nm}$.

\section{Gram scale preparation of 3ae and 3ag}

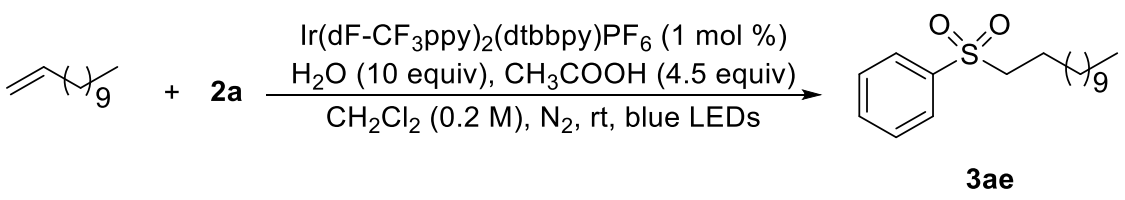

\section{Scheme S2}

A $50 \mathrm{~mL}$ flask equipped with a magnetic stirring bar and a rubber stopper was charged with dodec-1-ene $(0.887 \mathrm{~mL}, 4.0 \mathrm{mmol}), 2 \mathrm{a}(1.32 \mathrm{~g}, 6.6 \mathrm{mmol})$ and $\left[\operatorname{Ir}\left(\mathrm{dF}\left(\mathrm{CF}_{3}\right) \mathrm{ppy}\right)_{2}(\mathrm{dtbbpy})\right] \mathrm{PF}_{6}(45 \mathrm{mg}, 0.04 \mathrm{mmol}, 1 \mathrm{~mol} \%)$. The flask was evacuated and backfilled with nitrogen (three times), and into which was added $20 \mathrm{~mL}$ of DCM and $0.72 \mathrm{~mL}$ of $\mathrm{H}_{2} \mathrm{O}$, followed by $\mathrm{CH}_{3} \mathrm{COOH}(1.08 \mathrm{~g}, 18 \mathrm{mmol})$. The mixture was irradiated under stirring with a blue LED ribbon $(6 \mathrm{~W})$ for $8 \mathrm{~h}$. The reaction was then quenched with saturated $\mathrm{NaHCO}_{3}(24 \mathrm{~mL})$, and the aqueous phase was extracted with 
$\mathrm{CH}_{2} \mathrm{Cl}_{2}(3 \times 40 \mathrm{~mL})$. The combined organic phases were dried over anhydrous $\mathrm{Na}_{2} \mathrm{SO}_{4}$, and then concentrated under reduced pressure on a rotary evaporator. The residual was subjected to silica gel column chromatography (eluent: $\mathrm{EA} / \mathrm{PE}=1: 10$ ) to afford 3ae $(1.21 \mathrm{~g}, 98 \%)$.

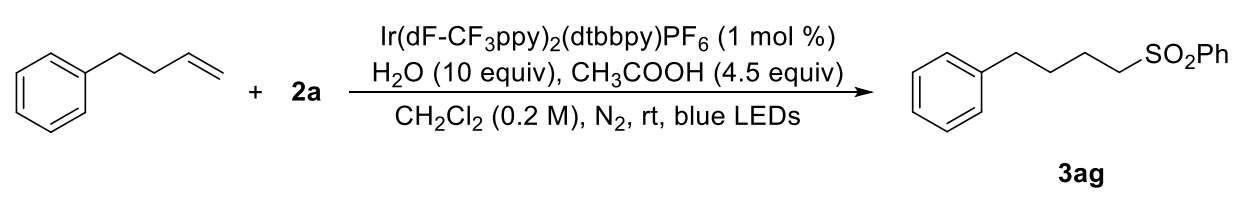

\section{Scheme S3}

A $50 \mathrm{~mL}$ flask equipped with a magnetic stirring bar and a rubber stopper was charged with but-3-en-1-ylbenzene $(0.6 \mathrm{~mL}, 4.0 \mathrm{mmol}), \mathbf{2 a}(1.32 \mathrm{~g}, 6.6 \mathrm{mmol})$ and $\left[\operatorname{Ir}\left(\mathrm{dF}\left(\mathrm{CF}_{3}\right) \mathrm{ppy}\right)_{2}(\mathrm{dtbbpy})\right] \mathrm{PF}_{6}(45 \mathrm{mg}, 0.04 \mathrm{mmol}, 1 \mathrm{~mol} \%)$. The flask was evacuated and backfilled with nitrogen (three times), and into which was added $20 \mathrm{~mL}$ of DCM and $0.72 \mathrm{~mL}$ of $\mathrm{H}_{2} \mathrm{O}$, followed by $\mathrm{CH}_{3} \mathrm{COOH}(1.08 \mathrm{~g}, 18 \mathrm{mmol})$. The mixture was irradiated under stirring with a blue LED ribbon $(6 \mathrm{~W})$ for $10 \mathrm{~h}$. The reaction was then quenched with saturated $\mathrm{NaHCO}_{3}(24 \mathrm{~mL})$, and the aqueous phase was extracted with $\mathrm{CH}_{2} \mathrm{Cl}_{2}(3 \times 40 \mathrm{~mL})$. The combined organic phases were dried over anhydrous $\mathrm{Na}_{2} \mathrm{SO}_{4}$, then concentrated under reduced pressure on a rotary evaporator, The residual was subjected to silica gel column chromatography (eluent: $\mathrm{EA} / \mathrm{PE}=1: 3$ ) to afford 3ag (1.06 g, 97\%).

\section{Optimization of reaction conditions}

Table S1. Screening of photocatalyst ${ }^{a}$

\begin{tabular}{|c|c|c|c|}
\hline & 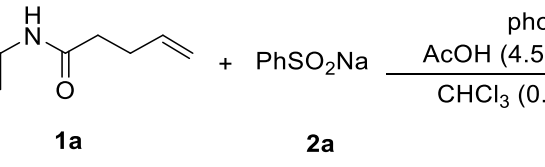 & $\begin{array}{l}2 \mathrm{~mol} \%) \\
\frac{\mathrm{H}_{2} \mathrm{O}(10 \text { equiv) }}{\text { olue LEDs, } \mathrm{N}_{2}, \mathrm{rt}}\end{array}$ & 3a \\
\hline entry & photocat & time (h) & yield of $\mathbf{3 a}(\%)^{b}$ \\
\hline 1 & {$\left[\operatorname{Ir}\left(\mathrm{dF}-\mathrm{CF}_{3} \mathrm{ppy}\right)_{2}(\mathrm{bpy})\right] \mathrm{PF}_{6}$} & 6 & 63 \\
\hline 2 & {$\left[\operatorname{Ir}(\mathrm{ppy})_{2}(\mathrm{dtbbpy})\right] \mathrm{PF}_{6}$} & 6 & 67 \\
\hline 3 & {$\left[\operatorname{Ir}(\mathrm{ppy})_{2}(\mathrm{bpy})\right] \mathrm{PF}_{6}$} & 6 & 48 \\
\hline 4 & {$\left[\operatorname{Ir}\left(\mathrm{dF}-\mathrm{CF}_{3} \mathrm{ppy}\right)_{2}\left(5,5-\mathrm{CF}_{3} \mathrm{bbpy}\right)\right] \mathrm{PF}_{6}$} & 10 & N.R. ${ }^{c}$ \\
\hline 5 & $\mathrm{Ru}(\mathrm{bpy})_{2} \mathrm{PF}_{6}$ & 10 & 66 \\
\hline 6 & Acr-Mes- $\mathrm{ClO}_{4}$ & 10 & $\mathbf{N} \cdot \mathbf{R}^{c}$ \\
\hline 7 & DCA & 10 & N.R. ${ }^{c}$ \\
\hline 8 & Rose Bengal & 10 & $\operatorname{trace}^{c}$ \\
\hline 9 & Eosin Y & 10 & N.R. ${ }^{c}$ \\
\hline
\end{tabular}

${ }^{a}$ The reactions were performed on a $0.2 \mathrm{mmol}$ scale under a nitrogen atmosphere. $0.33 \mathrm{mmol}$ of $2 \mathbf{a}$ $\left(\mathrm{PhSO}_{2} \mathrm{Na} \cdot 2 \mathrm{H}_{2} \mathrm{O}\right)$ and $2 \mathrm{~mol} \%$ of photocatalyst were used. ${ }^{b}$ Isolated yield. ${ }^{c}$ Most of 1a was recovered. DCA: 9,10-anthracenedicarbonitrile. 
Table S2. Effect of AcOH and $\mathrm{H}_{2} \mathrm{O}^{a}$

\begin{tabular}{|c|c|c|c|c|}
\hline & $\begin{array}{r}\mathrm{PhSC} \\
\mathbf{2}\end{array}$ & \multicolumn{2}{|c|}{$\begin{array}{l}{\left[\begin{array}{ll}\operatorname{lr}\left(\mathrm{dF}\left(\mathrm{CF}_{3}\right) \mathrm{pppy}_{2}(\mathrm{dtbbpy})\right] \mathrm{PF}_{6}(2 \mathrm{~mol} \%) \\
\mathrm{AcOH}(\mathrm{x} \text { equiv) }) \mathrm{H}_{2} \mathrm{O}(\mathrm{y} \text { equiv) }\end{array}\right.} \\
\underset{\mathrm{CHCl}_{3}(0.2 \mathrm{M}), \text { blue LEDs, } \mathrm{N}_{2} \text {, rt }}{\longrightarrow}\end{array}$} & $3 a$ \\
\hline entry & $\mathrm{AcOH}$ (x equiv) & $\mathrm{H}_{2} \mathrm{O}$ (y equiv) & time (h) & yield of $\mathbf{3 a}(\%)^{b}$ \\
\hline 1 & 0 & 0 & 10 & $\operatorname{trace}^{c}$ \\
\hline 2 & 4.0 & 0 & 4 & 77 \\
\hline 3 & 4.0 & 4 & 4 & 77 \\
\hline 4 & 4.0 & 10 & 4 & 94 \\
\hline 5 & 4.0 & $0.1(\mathrm{~mL})$ & 4 & 91 \\
\hline 6 & 4.0 & $0.5(\mathrm{~mL})$ & 5 & 79 \\
\hline 7 & 0 & 10 & 24 & N.R. ${ }^{c}$ \\
\hline 8 & 2.0 & 10 & 10 & 65 \\
\hline 9 & 2.5 & 10 & 10 & 70 \\
\hline 10 & 3.0 & 10 & 5 & 74 \\
\hline 11 & 3.5 & 10 & 4 & 87 \\
\hline 12 & 4.5 & 10 & 4 & 98 \\
\hline $13^{d}$ & 0 & 0 & 24 & N.R. ${ }^{c}$ \\
\hline $14^{e}$ & 0 & 0 & 24 & N.R. ${ }^{c}$ \\
\hline
\end{tabular}

${ }^{a}$ The reactions were performed on $0.2 \mathrm{mmol}$ scale under a nitrogen atmosphere. $0.33 \mathrm{mmol}$ of $\mathbf{2 a}$ $\left(\mathrm{PhSO}_{2} \mathrm{Na} \cdot 2 \mathrm{H}_{2} \mathrm{O}\right)$ and $2 \mathrm{~mol} \%$ of photocatalyst were used. ${ }^{b}$ Isolated yield. ${ }^{c}$ Most of $1 \mathbf{a}$ was recovered. ${ }^{d}$ The reaction was performed in $\mathrm{MeOH} .{ }^{e}$ The reaction was performed in EtOH.

Table S3. Effect of solvent ${ }^{a}$

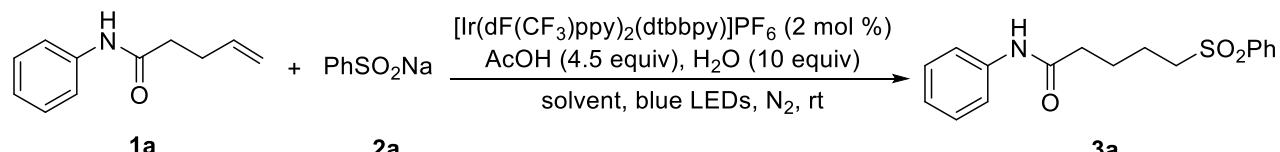

$1 \mathrm{a}$

2a

$3 a$

\begin{tabular}{cccc}
\hline entry & solvent & time $(\mathrm{h})$ & yield of $\mathbf{3 a}(\%)^{b}$ \\
\hline 1 & $\mathrm{CH}_{2} \mathrm{Cl}_{2}$ & 4 & 98 \\
2 & $\mathrm{CH}_{3} \mathrm{Cl}$ & 4 & 98 \\
3 & $\mathrm{BrCH}_{2} \mathrm{CH}_{2} \mathrm{Br}$ & 5 & 81 \\
4 & $\mathrm{EA}$ & 10 & $\operatorname{trace}^{c}$ \\
5 & $\mathrm{CH}_{3} \mathrm{COCH}$ & 10 & 14 \\
6 & $\mathrm{MeCN}$ & 10 & 11 \\
7 & $\mathrm{THF}$ & 10 & $\operatorname{trace}^{c}$ \\
8 & $\mathrm{DMF}$ & 10 & ${\mathrm{~N} . \mathrm{R}^{c}}^{c}$ \\
9 & $\mathrm{DMSO}$ & 10 & $\operatorname{trace}^{c}$ \\
10 & enthanol & 10 & $\operatorname{trace}^{c}$
\end{tabular}

${ }^{a}$ The reactions were performed on $0.2 \mathrm{mmol}$ scale. $0.33 \mathrm{mmol}$ of $2 \mathrm{a}\left(\mathrm{PhSO}_{2} \mathrm{Na} \cdot 2 \mathrm{H}_{2} \mathrm{O}\right)$ and $2 \mathrm{~mol} \%$ of photocatalyst were used. ${ }^{b}$ Isolated yield. ${ }^{c}$ Most of $\mathbf{1 a}$ was recovered. 
Table S4. Optimization of the amount of photocatalyst and $\mathrm{PhSO}_{2} \mathrm{Na}^{a}$

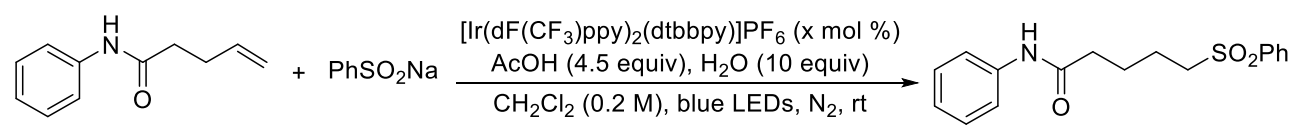

$1 \mathbf{a}$

$2 \mathbf{a}$

$3 a$

\begin{tabular}{ccccc}
\hline entry & equiv of $\mathbf{2 a}$ & $\mathrm{x}(\mathrm{mol} \%)$ & time $(\mathrm{h})$ & yield of $\mathbf{3 a}(\%)^{b}$ \\
\hline 1 & 1.31 & 2.0 & 5 & 68 \\
2 & 1.48 & 2.0 & 5 & 79 \\
3 & 1.65 & 2.0 & 4 & 98 \\
4 & 1.65 & 1.5 & 4 & 98 \\
5 & 1.65 & 1.0 & 4 & 98 \\
6 & 1.65 & 0.5 & 4 & 87 \\
7 & 1.65 & 0 & 10 & trace $^{c}$ \\
$8^{d}$ & 1.65 & 1.0 & 10 & N.R. ${ }^{c}$ \\
\hline
\end{tabular}

${ }^{a}$ The reactions were performed on $0.2 \mathrm{mmol}$ scale. ${ }^{b}$ Isolated yield. ${ }^{c}$ Most of $1 \mathbf{a}$ was recovered. ${ }^{d}$ In the dark.
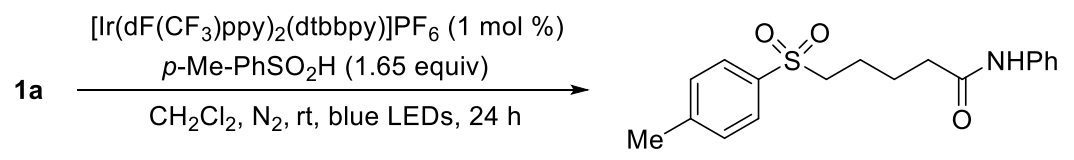

$10,39 \%$

$$
\begin{aligned}
& {\left[\operatorname{Ir}\left(\mathrm{dF}\left(\mathrm{CF}_{3}\right) \mathrm{ppy}\right)_{2} \text { (dtbbpy)]PF } 6\right. \text { (1 mol \%) }} \\
& \text { 1a } \underset{\mathrm{CH}_{2} \mathrm{Cl}_{2}, \mathrm{~N}_{2}, \mathrm{rt} \text {, blue LEDs, } 24 \mathrm{~h}}{\stackrel{p-\mathrm{Me}-\mathrm{PhSO}_{2} \mathrm{H}\left(1.65 \text { equiv), } \mathrm{H}_{2} \mathrm{O}(10 \text { equiv) }\right.}{\longrightarrow}} \quad \mathbf{1 0}, 70 \%
\end{aligned}
$$

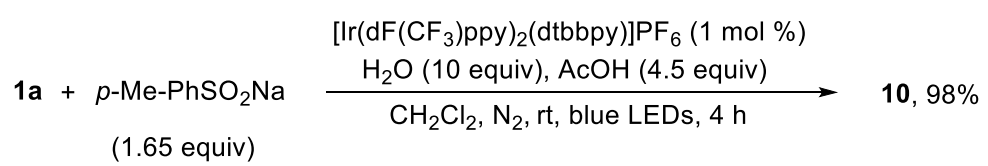

The reactions were performed on $0.2 \mathrm{mmol}$ scale in $1.0 \mathrm{~mL} \mathrm{CH}_{2} \mathrm{Cl}_{2}$.

\section{Scheme S4. Reaction of 1 a with $p-\mathrm{MePhSO}_{2} \mathrm{H}$ and $p-\mathrm{MePhSO}_{2} \mathrm{Na}$}

\section{Deuterium experiment}

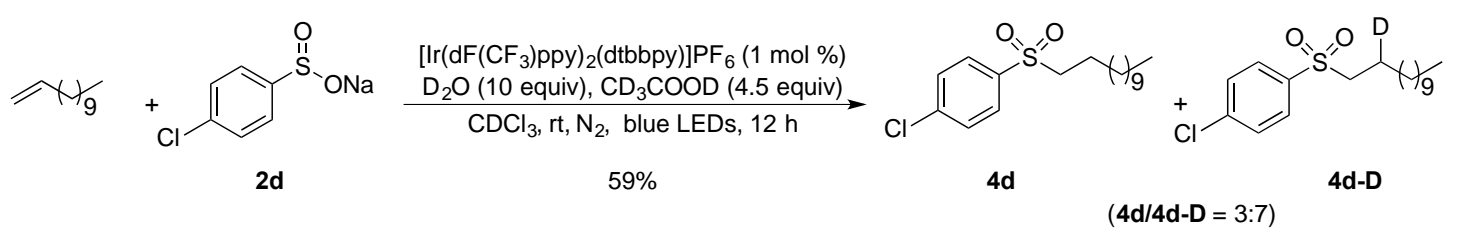

\section{Scheme S5}

A flame-dried glass tube equipped with a magnetic stirring bar and a rubber stopper was charged with dodec-1-ene $(111 \mu \mathrm{L}, 0.5 \mathrm{mmol}), 2 \mathrm{~d}(0.165 \mathrm{~g}, 0.825 \mathrm{mmol})$ and $\left[\operatorname{Ir}\left(\mathrm{dF}_{(}\left(\mathrm{CF}_{3}\right) \mathrm{ppy}\right)_{2}(\mathrm{dtbbpy})\right] \mathrm{PF}_{6}(5.6 \mathrm{mg}, 0.005 \mathrm{mmol}, 1 \mathrm{~mol} \%)$. The tube was 
evacuated and backfilled with nitrogen (three times), and into which was added 2.5 $\mathrm{mL}$ of $\mathrm{CDCl}_{3}$ and $100 \mu \mathrm{L}$ of $\mathrm{D}_{2} \mathrm{O}$, followed by $\mathrm{CD}_{3} \mathrm{COOD}(0.144 \mathrm{~g}, 2.25 \mathrm{mmol})$. The mixture was irradiated under stirring with blue LED lamps (6W) for $12 \mathrm{~h}$, then concentrated under the reduced pressure on a rotary evaporator, and the residual was subjected to silica gel column chromatography (eluent: $\mathrm{EA} / \mathrm{PE}=1: 10$ ) to afford $\mathbf{4 d}$ and 4d-D (0.102 g, 59\%).
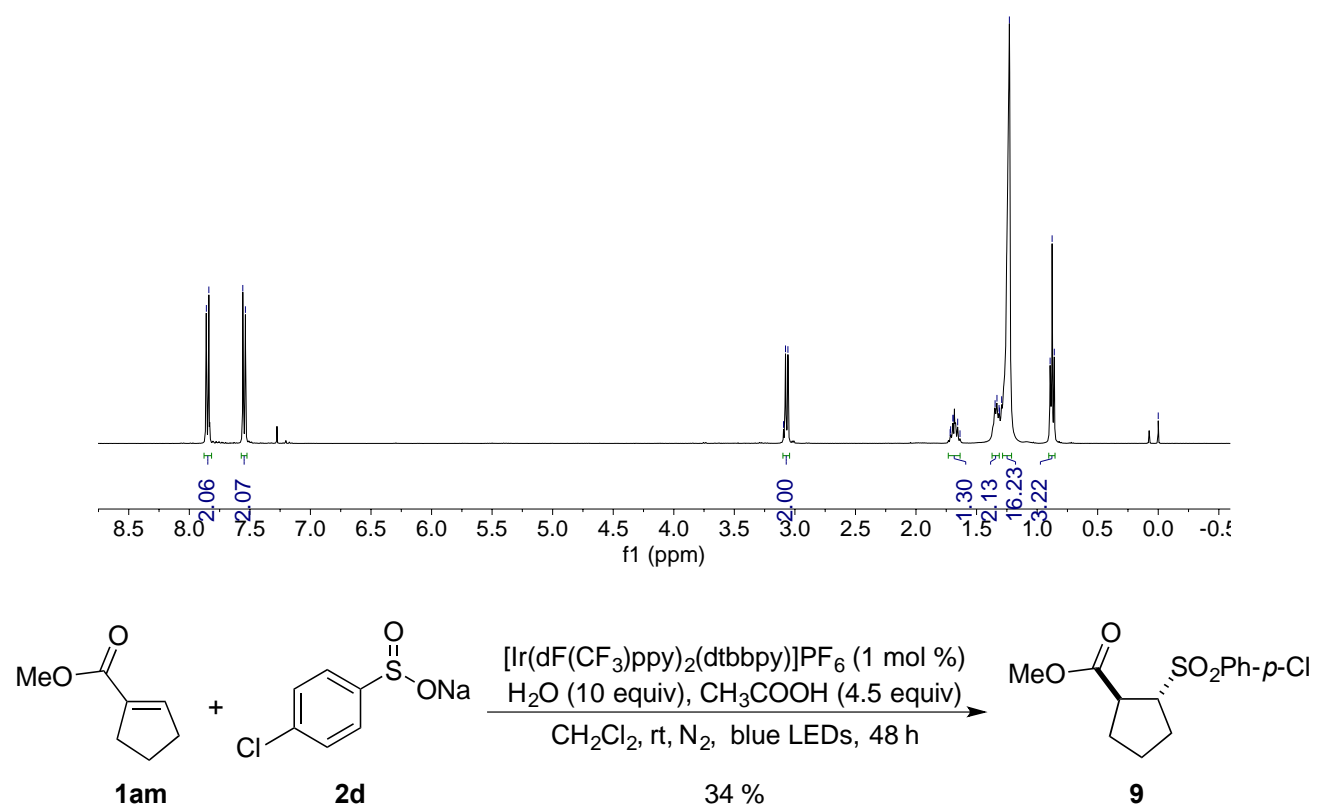

Scheme S6

A flame-dried glass tube equipped with a magnetic stirring bar and a rubber stopper was charged with 1am $(61 \mu \mathrm{L}, 0.5 \mathrm{mmol}), \mathbf{2 d}(0.165 \mathrm{~g}, 0.825 \mathrm{mmol})$ and $\left[\operatorname{Ir}\left(\mathrm{dF}\left(\mathrm{CF}_{3}\right) \mathrm{ppy}\right)_{2}(\mathrm{dtbbpy})\right] \mathrm{PF}_{6}(5.6 \mathrm{mg}, 0.005 \mathrm{mmol}, 1 \mathrm{~mol} \%)$. The tube was evacuated and backfilled with nitrogen (three times), and into which was added 2.5 $\mathrm{mL}$ of $\mathrm{CH}_{2} \mathrm{Cl}_{2}$ and $90 \mu \mathrm{L}$ of $\mathrm{H}_{2} \mathrm{O}$, followed by $\mathrm{CH}_{3} \mathrm{COOH}(0.135 \mathrm{~g}, 2.25 \mathrm{mmol})$. The mixture was irradiated under stirring with blue LED lamps $(6 \mathrm{~W})$ for $48 \mathrm{~h}$, and then concentrated under the reduced pressure on a rotary evaporator. The residual was subjected to silica gel column chromatography (eluent: $\mathrm{EA} / \mathrm{PE}=1: 3$ ) to afford 9 $(0.051 \mathrm{~g}, 34 \%)$. 

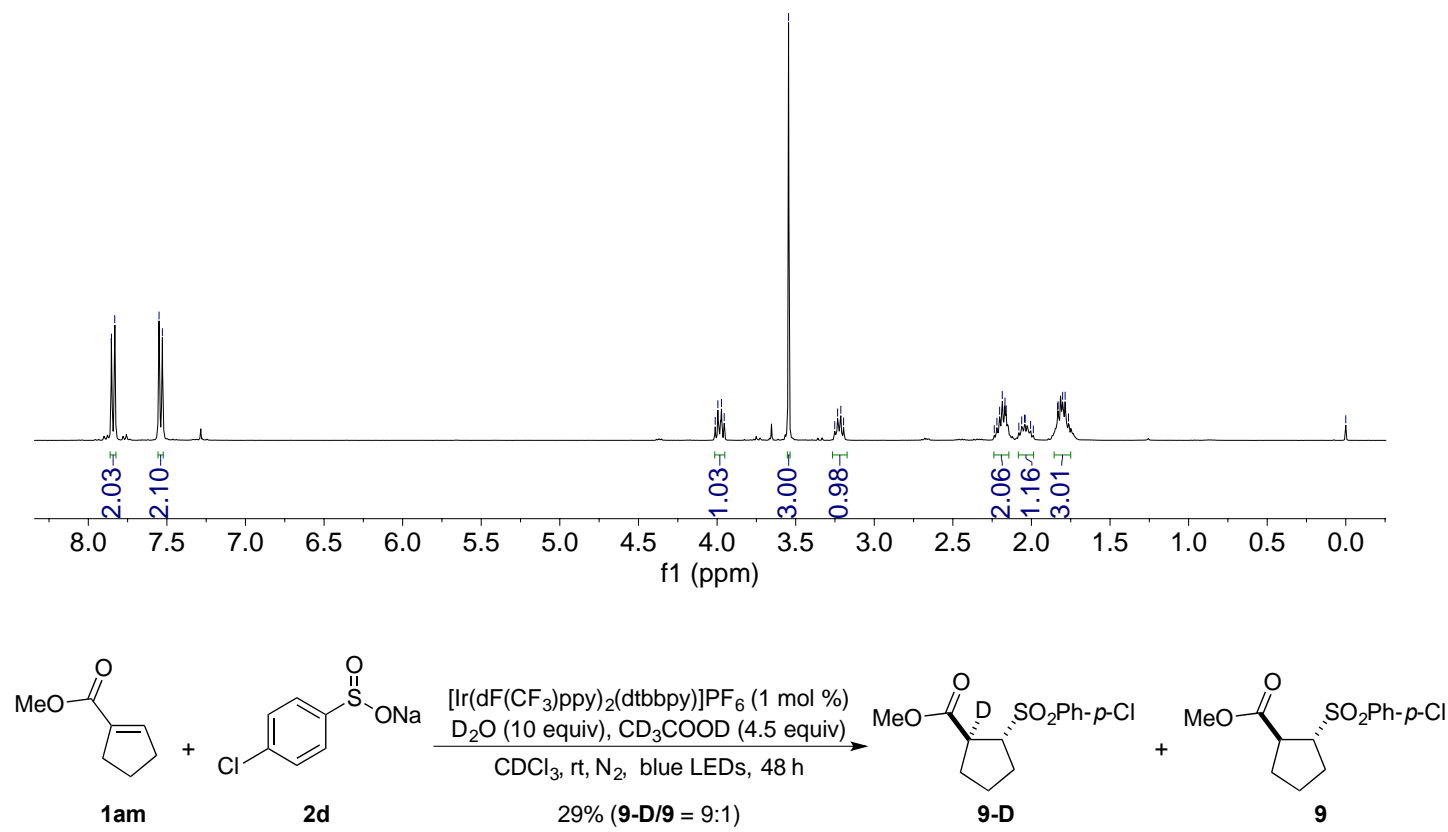

Scheme S7

A flame-dried glass tube equipped with a magnetic stirring bar and a rubber stopper was charged with 1am (61 $\mu \mathrm{L}, 0.5 \mathrm{mmol}), 2 \mathrm{2d}(0.165 \mathrm{~g}, 0.825 \mathrm{mmol})$ and $\left[\operatorname{Ir}\left(\mathrm{dF}\left(\mathrm{CF}_{3}\right) \mathrm{ppy}\right)_{2}(\mathrm{dtbbpy})\right] \mathrm{PF}_{6}(5.6 \mathrm{mg}, 0.005 \mathrm{mmol}, 1 \mathrm{~mol} \%)$. The tube was evacuated and backfilled with nitrogen (three times), and into which was added 2.5 $\mathrm{mL}$ of $\mathrm{CDCl}_{3}$ and $100 \mu \mathrm{L}$ of $\mathrm{D}_{2} \mathrm{O}$, followed by $\mathrm{CD}_{3} \mathrm{COOD}(0.144 \mathrm{~g}, 2.25 \mathrm{mmol})$. The mixture was irradiated under stirring with blue LED lamps (6 W) for $48 \mathrm{~h}$, and then concentrated under the reduced pressure on a rotary evaporator. The residual was subjected to silica gel column chromatography (eluent: $\mathrm{EA} / \mathrm{PE}=1: 3$ ) to afford 9 and 9-D $(0.043 \mathrm{~g}, 29 \%)$. 


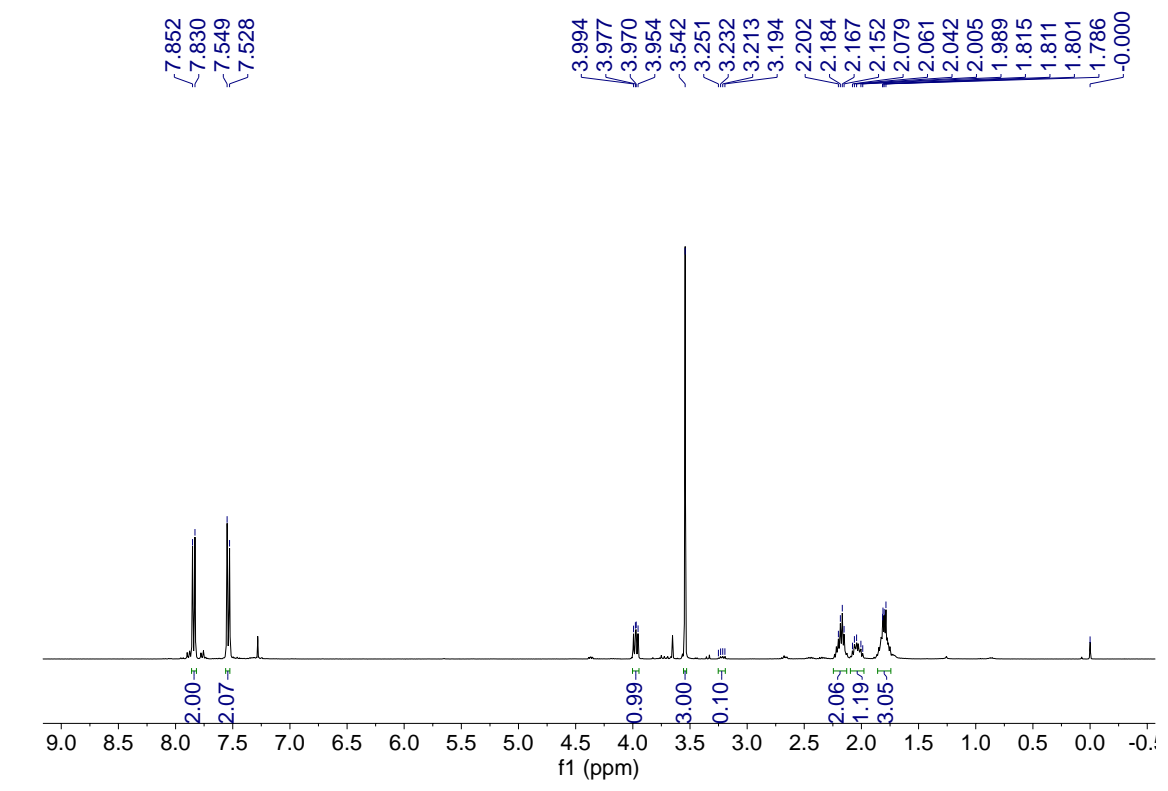

\section{Quantum yield measurement}

The quantum yield measurement was performed according to the procedures as described by Yoon, ${ }^{6}$ Ritter, ${ }^{7}$ Aleman ${ }^{8}$ and Glorious. ${ }^{9}$

\section{(1) Solution preparation}

Potassium ferrioxalate solution $(0.012 \mathrm{M}): 59.0 \mathrm{mg}$ of $\mathrm{K}_{3}\left[\mathrm{Fe}\left(\mathrm{C}_{2} \mathrm{O}_{4}\right)\right] \cdot 3 \mathrm{H}_{2} \mathrm{O}$ and $28 \mu \mathrm{L}$ of $\mathrm{H}_{2} \mathrm{SO}_{4}$ were added into a $10 \mathrm{~mL}$ brown volumetric flask and filled to the mark with ultra-pure water.

1,10-Phenanthroline solution $(0.01 \mathrm{M}): 29.0 \mathrm{mg}$ of 1,10-phenantroline monohydrate was added into a $10 \mathrm{~mL}$ brown volumetric flask and filled to the mark with ultra-pure water.

NaOAc and HOAc buffer solution: $1.235 \mathrm{~g}$ of $\mathrm{NaOAc}$ and $250 \mu \mathrm{L}$ of $\mathrm{H}_{2} \mathrm{SO}_{4}$ were added into a $25 \mathrm{~mL}$ volumetric flask and filled to the mark with ultra-pure water.

$\left[\operatorname{Ir}\left(d F\left(C F_{3}\right) p p y\right)_{2}(d t b b p y)\right] P F_{6} \quad$ in $\quad D C M \quad(0.0001 M): \quad 5.6 \quad \mathrm{mg}$ of

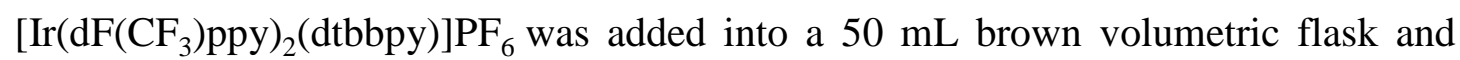
filled to the mark with dry DCM.

\section{All solutions were prepared and stored in the dark.}

\section{(2) Determination of the light intensity at $455 \mathrm{~nm}$}

$2.0 \mathrm{~mL}$ of $0.012 \mathrm{M}$ potassium ferrioxalate solution was added into the reaction vial, and irradiated with blue LED lamps $(6 \mathrm{~W})$ for 90 second. After that, $0.1 \mathrm{~mL}$ of this solution was taken as an aliquot. To each aliquot, $2.0 \mathrm{~mL}$ of the buffer solution and $0.5 \mathrm{~mL}$ of the 1,10-phenanthroline solution were added with a syringe, and the mixture was stirred in the dark for $1 \mathrm{~h}$. The mixture was then diluted in a $10 \mathrm{~mL}$ brown volumetric flask with ultra-pure water. The absorbance of the resulting solution in a quartz cuvette $(1 \times 1 \mathrm{~cm})$ at $510 \mathrm{~nm}$ was measured with a UV-Vis 
spectrometer. A non-irradiated sample was also prepared in the same manner otherwise, and the absorbance at $510 \mathrm{~nm}$ was measured.

The amount of ferrous ion formed was calculated as following:

$\mathrm{mol}$ of $\mathrm{Fe}^{2+}=\frac{\mathrm{V}_{1} \times \mathrm{V}_{3} \times \mathrm{A}}{\mathrm{V}_{2} \times \mathrm{i} \times \mathrm{e}}=\frac{0.002 \mathrm{~L} \times 0.010 \mathrm{~L} \times 1.0034}{0.0001 \mathrm{~L} \times 1.0 \mathrm{~cm} \times 11,100 \mathrm{~L} / \mathrm{mol} / \mathrm{cm}}=1.8 \times 10^{-5} \mathrm{~mol}$

where $\mathrm{V}_{1}$ is the irradiated volume $(0.002 \mathrm{~L}), \mathrm{V}_{2}$ is the irradiated volume $(0.0001 \mathrm{~L})$, $\mathrm{V}_{3}$ is the irradiated volume $(0.010 \mathrm{~L}), \Delta \mathrm{A}$ is the difference in absorbance at $510 \mathrm{~nm}$ between the irradiated and non-irradiated samples, 1 is the path length $(1.00 \mathrm{~cm})$, and $\varepsilon$ is the molar absorptivity at $510 \mathrm{~nm}(11,100 \mathrm{~L} / \mathrm{mol} \cdot \mathrm{cm})$.

$$
\text { photon flux }=\frac{\mathrm{mol} \text { of } \mathrm{Fe}^{2+}}{\varnothing \times \mathrm{t} \times \mathrm{f}}=\frac{1.8 \times 10^{-5} \mathrm{~mol}}{1.12 \times 90 \mathrm{~s} \times 0.3715}=4.8 \times 10^{-7} \text { einstein } / \mathrm{s}
$$

where $\Phi$ is the quantum yield for the ferrioxalate actinometer (approximated as 1.12, which was reported for a $0.01 \mathrm{M}$ solution at $\lambda=458 \mathrm{~nm})^{10}, \mathrm{t}$ is the time $(90.0 \mathrm{~s})$, and $\mathrm{f}$ is the fraction of light absorbed at $455 \mathrm{~nm}$ (as shown in Figure S1). The fraction of light absorbed was determined by the following equation:

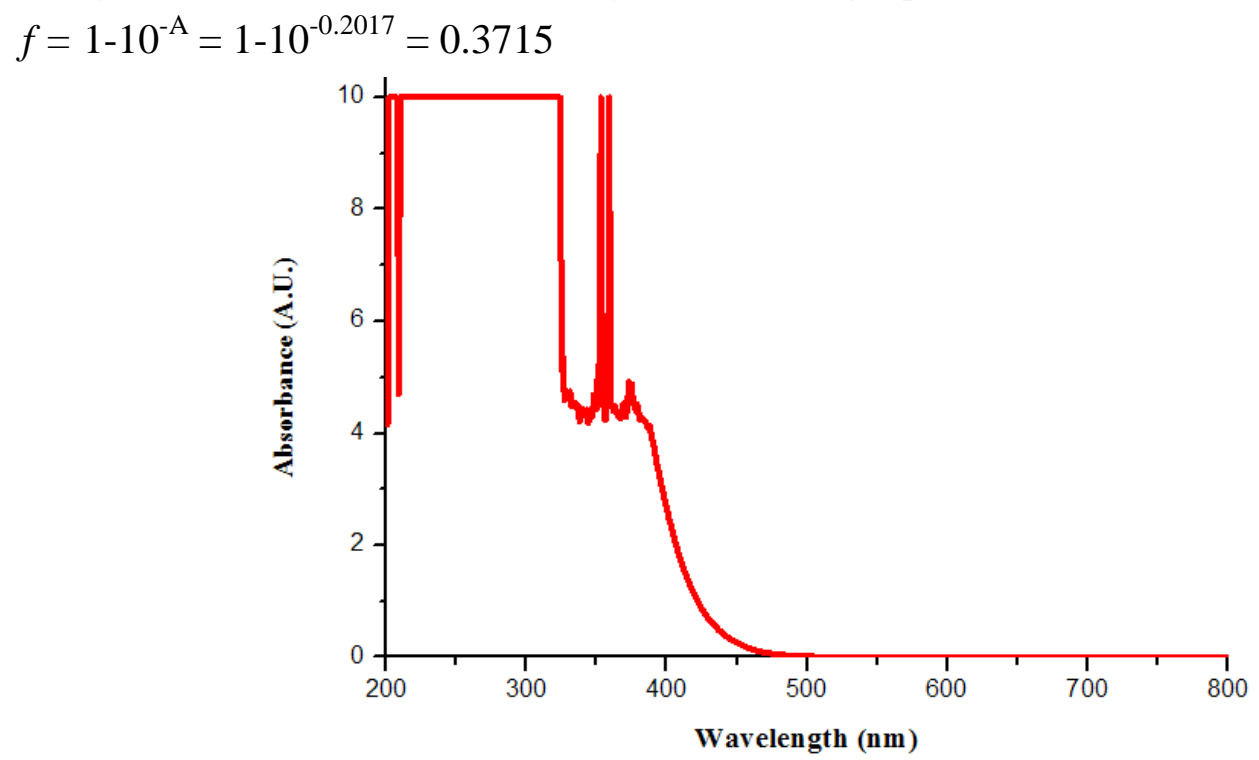

Figure S1. Absorbance of the ferrioxalate actinometer solution.

\section{(3) Determination of reaction quantum yield at $455 \mathrm{~nm}$}

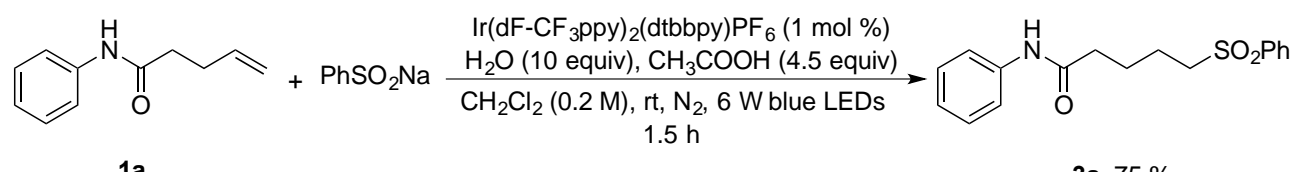

$1 a$

3a, $75 \%$

\section{Scheme S8}

A flame-dried glass tube equipped with a magnetic stir bar and a rubber stopper was charged with $1 \mathrm{a}(0.035 \mathrm{~g}, 0.2 \mathrm{mmol}), \mathrm{PhSO}_{2} \mathrm{Na} \cdot 2 \mathrm{H}_{2} \mathrm{O}(0.066 \mathrm{~g}, 0.33 \mathrm{mmol})$ and $\left[\operatorname{Ir}\left(\mathrm{dF}\left(\mathrm{CF}_{3}\right) \mathrm{ppy}\right)_{2}(\mathrm{dtbbpy})\right] \mathrm{PF}_{6}(2.2 \mathrm{mg}, 0.002 \mathrm{mmol}, 1 \mathrm{~mol} \%)$. The tube was 
evacuated and backfilled with nitrogen (three times), and into which was added 1.0 $\mathrm{mL}$ of DCM and $36 \mu \mathrm{L}$ of $\mathrm{H}_{2} \mathrm{O}$, followed by $\mathrm{CH}_{3} \mathrm{COOH}(0.054 \mathrm{~g}, 0.9 \mathrm{mmol})$. The vial was put at $2.0 \mathrm{~cm}$ away from blue LED lamps $(6 \mathrm{~W})$ and stirred under irradiation at room temperature for 5400 second. After irradiation, the mixture was passed through a short plug of silica gel. The yield of the product 3a was determined to be $75 \%$ (corresponding to $1.5 \times 10^{-4} \mathrm{~mol}$ ) by ${ }^{1} \mathrm{H}$ NMR based on a 1,3,5-trimethaxybenzene internal standard. A $1 \times 10^{-4} \mathrm{M}$ solution of $\left[\operatorname{Ir}\left(\mathrm{dF}\left(\mathrm{CF}_{3}\right) \mathrm{ppy}\right)_{2}(\mathrm{dtbbpy})\right] \mathrm{PF}_{6}$ in $\mathrm{DCM}$ was prepared, and the absorbance of the solution at $455 \mathrm{~nm}$ was measured $(\mathrm{A}=0.0405)$. The fraction of light absorbed at $455 \mathrm{~nm}$ (as shown in Figure S2) was calculated as described above $(f=0.089)$.

The quantum yield was calculated as follows:

$$
\varnothing=\frac{\text { mol of product } 3 \mathrm{a}}{\text { photo flux } \times \mathrm{t} \times \mathrm{f}}=\frac{15 \times 10^{-5} \mathrm{~mol}}{4.8 \times 10^{-7} \text { einstein } / \mathrm{s} \times 5400 \mathrm{~s} \times 0.089}=0.65
$$

Where the mol of product 3a was $1.5 \times 10^{-4} \mathrm{~mol}$, photo flux is determined by the ferrioxalate actinometer $\left(4.8 \times 10^{-7}\right.$ einstein $\left.\cdot \mathrm{s}^{-1}\right), \mathrm{t}$ is the irradiated time $(5400 \mathrm{~s})$, and $\mathrm{f}$ is the fraction of light absorbed by $\left[\operatorname{Ir}\left(\mathrm{dF}\left(\mathrm{CF}_{3}\right) \text { ppy }\right)_{2}(\mathrm{dtbbpy})\right] \mathrm{PF}_{6}$ at $455 \mathrm{~nm}(0.0405)$.

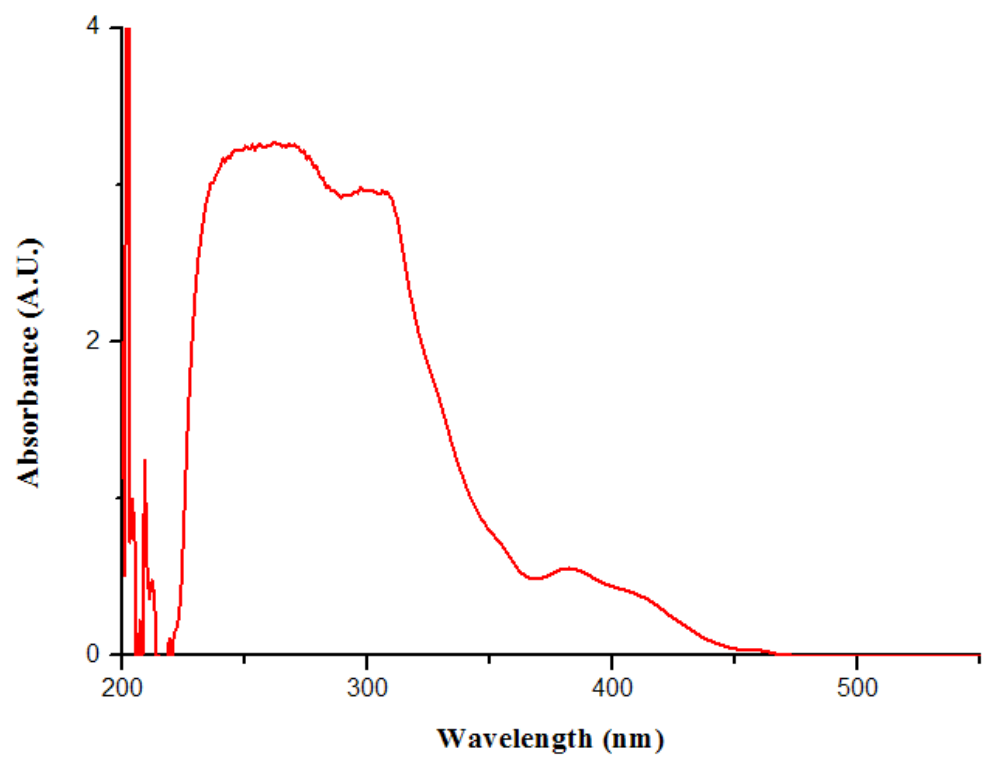

Figure S2. Absorbance of a $1.0 \times 10^{-4} \mathrm{M}$ solution of $\left[\operatorname{Ir}\left(\mathrm{dF}\left(\mathrm{CF}_{3}\right) \mathrm{ppy}\right)_{2}(\mathrm{dtbbpy})\right] \mathrm{PF}_{6}$ in $\mathrm{DCM}$.

\section{Characterization data}<smiles>C=CCCC(=O)N(CC)c1ccccc1</smiles>

$N$-Ethyl- $N$-phenylpent-4-enamide (1j) 
Colorless oil (3.1 mmol scale, FCC eluent: EA/PE $=1: 3,0.43 \mathrm{~g}$, yield: $68 \%), \mathrm{R}_{\mathrm{f}}=$ $0.47(\mathrm{EA} / \mathrm{PE}=1: 3) ;{ }^{1} \mathrm{H} \mathrm{NMR}\left(400 \mathrm{MHz}, \mathrm{CDCl}_{3}\right) \delta 7.43(\mathrm{t}, J=7.2 \mathrm{~Hz}, 2 \mathrm{H}), 7.35(\mathrm{t}, J$ $=7.2 \mathrm{~Hz}, 1 \mathrm{H}), 7.17-7.15(\mathrm{~m}, 2 \mathrm{H}), 5.77-5.67(\mathrm{~m}, 1 \mathrm{H}), 4.95-4.88(\mathrm{~m}, 2 \mathrm{H}), 3.76(\mathrm{q}, J=$ $7.2 \mathrm{~Hz}, 2 \mathrm{H}), 2.35-2.30(\mathrm{~m}, 2 \mathrm{H}), 2.11(\mathrm{t}, J=7.2 \mathrm{~Hz}, 2 \mathrm{H}), 1.11(\mathrm{t}, J=7.2 \mathrm{~Hz}, 3 \mathrm{H}) ;{ }^{13} \mathrm{C}$ NMR (100 MHz, $\left.\mathrm{CDCl}_{3}\right) \delta 171.4,142.1,137.3,129.4,128.2,127.6,114.7,43.7,33.6$, 29.2, 12.9; HRMS (ESI): Calcd for $\mathrm{C}_{13} \mathrm{H}_{18} \mathrm{NO}$ [M+H]: 204.1383, found: 204.1381.

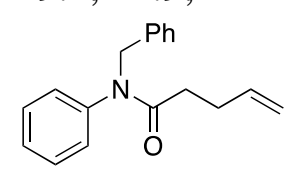

\section{$N$-Benzyl- $N$-phenylpent-4-enamide (1k)}

Colorless oil (3.1 mmol scale, FCC eluent: $\mathrm{EA} / \mathrm{PE}=1: 3,0.62 \mathrm{~g}$, yield: $75 \%$ ), $\mathrm{R}_{\mathrm{f}}=$ $0.47(\mathrm{EA} / \mathrm{PE}=1: 3) ;{ }^{1} \mathrm{H}$ NMR $\left(400 \mathrm{MHz}, \mathrm{CDCl}_{3}\right) \delta 7.33-7.27(\mathrm{~m}, 3 \mathrm{H}), 7.25-7.21(\mathrm{~m}$, $3 \mathrm{H}), 7.20-7.18(\mathrm{~m}, 2 \mathrm{H}), 6.97-6.95(\mathrm{~m}, 2 \mathrm{H}), 5.79-5.69(\mathrm{~m}, 1 \mathrm{H}), 4.97-4.89(\mathrm{~m}, 4 \mathrm{H})$, 2.37 (q, J = 7.6 Hz, 2H), 2.18-2.14 (m, 2H); ${ }^{13} \mathrm{C} \mathrm{NMR}\left(100 \mathrm{MHz}, \mathrm{CDCl}_{3}\right) \delta 171.9$, 142.2, 137.4, 137.3, 129.4, 128.7, 128.3, 128.2, 127.8, 127.2, 114.9, 52.8, 33.5, 29.3;

HRMS (ESI): Calcd for $\mathrm{C}_{18} \mathrm{H}_{20} \mathrm{NO}[\mathrm{M}+\mathrm{H}]: 266.1539$, found: 266.1539 .

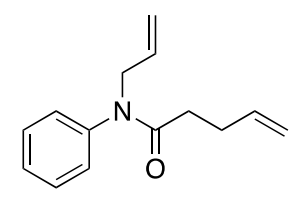

\section{$N$-Allyl- $N$-phenylpent-4-enamide (11)}

Colorless oil (3.1 mmol scale, FCC eluent: $\mathrm{EA} / \mathrm{PE}=1: 5,0.48 \mathrm{~g}$, yield: $72 \%), \mathrm{R}_{\mathrm{f}}=$ $0.55(\mathrm{EA} / \mathrm{PE}=1: 3) ;{ }^{1} \mathrm{H}$ NMR $\left(400 \mathrm{MHz}, \mathrm{CDCl}_{3}\right) \delta 7.40(\mathrm{t}, \mathrm{J}=7.6 \mathrm{~Hz}, 2 \mathrm{H}), 7.33(\mathrm{t}, \mathrm{J}$ $=7.2 \mathrm{~Hz}, 1 \mathrm{H}), 7.16(\mathrm{~d}, \mathrm{~J}=7.6 \mathrm{~Hz}, 2 \mathrm{H}), 5.91-5.82(\mathrm{~m}, 1 \mathrm{H}), 5.78-5.68(\mathrm{~m}, 1 \mathrm{H}), 5.11-$ $5.04(\mathrm{~m}, 2 \mathrm{H}), 4.96-4.89(\mathrm{~m}, 2 \mathrm{H}), 4.30(\mathrm{~d}, \mathrm{~J}=6.4 \mathrm{~Hz}, 2 \mathrm{H}), 2.34(\mathrm{q}, \mathrm{J}=7.2 \mathrm{~Hz}, 2 \mathrm{H})$, $2.15(\mathrm{t}, \mathrm{J}=7.2 \mathrm{~Hz}, 2 \mathrm{H}) ;{ }^{13} \mathrm{C} \mathrm{NMR}\left(100 \mathrm{MHz}, \mathrm{CDCl}_{3}\right) \delta 171.6,142.2,137.2,133.0$, 129.4, 128.1, 127.7, 117.5, 114.8, 52.0, 33.5, 29.2; HRMS (ESI): Calcd for $\mathrm{C}_{14} \mathrm{H}_{18} \mathrm{NO}[\mathrm{M}+\mathrm{H}]: 216.1383$, found: 216.1372 .<smiles>C=CCCOC(=O)c1cccn1C</smiles>

\section{But-3-en-1-yl 1-methyl-1H-pyrrole-2-carboxylate (1v)}

Colorless oil (10 mmol scale, FCC eluent: EA/PE = 1:10, $1.56 \mathrm{~g}$, yield: $87 \%$ ), $\mathrm{R}_{\mathrm{f}}=$ $0.68(\mathrm{EA} / \mathrm{PE}=1: 3) ;{ }^{1} \mathrm{H} \mathrm{NMR}\left(400 \mathrm{MHz}, \mathrm{CDCl}_{3}\right) \delta 6.94(\mathrm{dd}, \mathrm{J}=4.0,2.0 \mathrm{~Hz}, 1 \mathrm{H})$, $6.75(\mathrm{t}, \mathrm{J}=2.4 \mathrm{~Hz}, 1 \mathrm{H}), 6.09(\mathrm{dd}, \mathrm{J}=4.0,2.4 \mathrm{~Hz}, 1 \mathrm{H}), 5.90-5.79(\mathrm{~m}, 1 \mathrm{H}), 5.16-5.07$ $(\mathrm{m}, 2 \mathrm{H}), 4.26(\mathrm{t}, \mathrm{J}=6.8 \mathrm{~Hz}, 2 \mathrm{H}), 3.90(\mathrm{~s}, 3 \mathrm{H}), 2.46(\mathrm{q}, \mathrm{J}=6.8 \mathrm{~Hz}, 2 \mathrm{H}) ;{ }^{13} \mathrm{C} \mathrm{NMR}$ $\left(100 \mathrm{MHz}, \mathrm{CDCl}_{3}\right) \delta 161.1,134.1,129.3,122.4,117.7,117.0,107.7,62.7,36.7,33.2$; HRMS (ESI): Calcd for $\mathrm{C}_{10} \mathrm{H}_{13} \mathrm{NO}_{2} \mathrm{Na}$ [M+Na]: 202.0838, found: 202.0835 . 


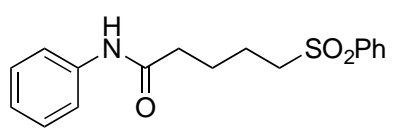

\section{$N$-Phenyl-5-(phenylsulfonyl)pentanamide (3a)}

White solid (Reaction time: $4 \mathrm{~h}$, FCC eluent: EA/PE = 1:1, $155 \mathrm{mg}$, yield: 98\%), mp: $115-117^{\circ} \mathrm{C} ; v 1303,1146,898,757 \mathrm{~cm}^{-1} ;{ }^{1} \mathrm{H}$ NMR $\left(400 \mathrm{MHz}, \mathrm{CDCl}_{3}\right) \delta 8.16(\mathrm{~s}, 1 \mathrm{H})$, $7.86(\mathrm{~d}, \mathrm{~J}=7.6 \mathrm{~Hz}, 2 \mathrm{H}), 7.62(\mathrm{t}, \mathrm{J}=7.6 \mathrm{~Hz}, 1 \mathrm{H}), 7.54-7.47(\mathrm{~m}, 4 \mathrm{H}), 7.24(\mathrm{t}, \mathrm{J}=8.0$ $\mathrm{Hz}, 2 \mathrm{H}), 7.05(\mathrm{t}, \mathrm{J}=7.6 \mathrm{~Hz}, 1 \mathrm{H}), 3.09(\mathrm{t}, \mathrm{J}=7.2 \mathrm{~Hz}, 2 \mathrm{H}), 2.31(\mathrm{~d}, \mathrm{~J}=6.8 \mathrm{~Hz}, 2 \mathrm{H})$, 1.76-1.75 (m, 4H); ${ }^{13} \mathrm{C}$ NMR (100 MHz, $\left.\mathrm{CDCl}_{3}\right) \delta 170.7,138.7,137.9,133.7,129.3$, 128.7, 127.7, 124.0, 119.9, 55.6, 36.3, 23.9, 22.0; HRMS (ESI): Calcd for $\mathrm{C}_{17} \mathrm{H}_{20} \mathrm{NO}_{3} \mathrm{~S}[\mathrm{M}+\mathrm{H}]: 318.1158$, found: 318.1158 .

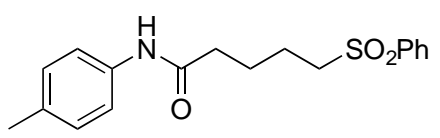

\section{5-(Phenylsulfonyl)- $N$-( $p$-tolyl)pentanamide (3b)}

White solid (Reaction time: $6 \mathrm{~h}$, FCC eluent: EA/PE $=1: 2,155 \mathrm{mg}$, yield: 94\%), mp: $128-130{ }^{\circ} \mathrm{C}$; IR (KBr) v 1297, 1244, 818, $732 \mathrm{~cm}^{-1} ;{ }^{1} \mathrm{H}$ NMR (400 MHz, $\left.\mathrm{CDCl}_{3}\right) \delta$ 8.09 (s, 1H), 7.86 (d, J = 7.6 Hz, 2H), 7.62 (t, J = 7.6 Hz, 1H), 7.52 (t, J = 7.6 Hz, 2H), $7.35(\mathrm{~d}, \mathrm{~J}=8.0 \mathrm{~Hz}, 2 \mathrm{H}), 7.04(\mathrm{~d}, \mathrm{~J}=8.4 \mathrm{~Hz}, 2 \mathrm{H}), 3.11-3.07(\mathrm{~m}, 2 \mathrm{H}), 2.31-2.27(\mathrm{~m}$, 5H), 1.75-1.74 (m, 4H); ${ }^{13} \mathrm{C}$ NMR $\left(100 \mathrm{MHz}, \mathrm{CDCl}_{3}\right) \delta 170.5,138.7,135.3,133.7$, 133.6, 129.2, 129.1, 127.7, 120.0, 55.6, 36.2, 23.9, 22.0, 20.7; HRMS (ESI): Calcd for $\mathrm{C}_{18} \mathrm{H}_{22} \mathrm{NO}_{3} \mathrm{~S}[\mathrm{M}+\mathrm{H}]: 332.1215$, found: 332.1217 .

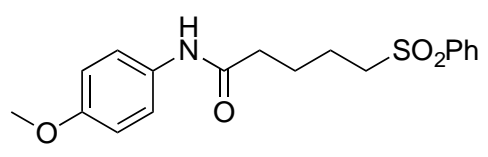

\section{$N$-(4-Methoxyphenyl)-5-(phenylsulfonyl)pentanamide (3c)}

White solid (Reaction time: $6 \mathrm{~h}$, FCC eluent: EA/PE $=1: 2,160 \mathrm{mg}$, yield: 92\%), mp: $111-113{ }^{\circ} \mathrm{C}$; IR (KBr) v 1297, 1244, 831, $748 \mathrm{~cm}^{-1}$; ${ }^{1} \mathrm{H}$ NMR (400 MHz, $\left.\mathrm{CDCl}_{3}\right) \delta$ $7.88(\mathrm{~d}, J=7.2 \mathrm{~Hz}, 2 \mathrm{H}), 7.75(\mathrm{~s}, 1 \mathrm{H}), 7.64(\mathrm{t}, J=7.2 \mathrm{~Hz}, 1 \mathrm{H}), 7.54(\mathrm{t}, J=7.6 \mathrm{~Hz}, 2 \mathrm{H})$, $7.37(\mathrm{~d}, J=8.8 \mathrm{~Hz}, 2 \mathrm{H}), 6.81(\mathrm{~d}, J=8.8 \mathrm{~Hz}, 2 \mathrm{H}), 3.76(\mathrm{~s}, 3 \mathrm{H}), 3.11(\mathrm{~d}, J=6.0 \mathrm{~Hz}$, 2H), 2.33-2.29 (m, 2H), 1.79-1.76 (m, 4H); $\left.{ }^{13} \mathrm{C} \mathrm{NMR} \mathrm{(100} \mathrm{MHz,} \mathrm{CDCl}_{3}\right) \delta 170.3$, 156.3, 138.9, 133.8, 131.0, 129.3, 127.9, 121.8, 114.0, 55.8, 55.4, 36.3, 24.0, 22.1; HRMS (ESI): Calcd for $\mathrm{C}_{18} \mathrm{H}_{22} \mathrm{NO}_{4} \mathrm{~S}$ [M+H] 348.1264, found: 348.1266 .

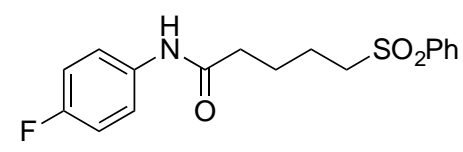

\section{$N$-(4-Fluorophenyl)-5-(phenylsulfonyl)pentanamide (3d)}

White solid (Reaction time: 6 h, FCC eluent: EA/PE = 1:2, $161 \mathrm{mg}$, yield: 96\%), mp: $136-138{ }^{\circ} \mathrm{C}$; IR $(\mathrm{KBr}) \vee 1296,1243,835,732 \mathrm{~cm}^{-1} ;{ }^{1} \mathrm{H}$ NMR $\left(400 \mathrm{MHz}, \mathrm{CDCl}_{3}\right) \delta$ $8.17(\mathrm{~s}, 1 \mathrm{H}), 7.88-7.86(\mathrm{~m}, 2 \mathrm{H}), 7.64(\mathrm{~d}, \mathrm{~J}=7.6 \mathrm{~Hz}, 1 \mathrm{H}), 7.54(\mathrm{t}, \mathrm{J}=7.6 \mathrm{~Hz}, 2 \mathrm{H})$, 
$7.43(\mathrm{dd}, \mathrm{J}=8.8,4.8 \mathrm{~Hz}, 2 \mathrm{H}), 6.93(\mathrm{t}, \mathrm{J}=8.4 \mathrm{~Hz}, 2 \mathrm{H}), 3.12(\mathrm{~d}, \mathrm{~J}=7.6 \mathrm{~Hz}, 2 \mathrm{H}), 2.33$ $(\mathrm{t}, \mathrm{J}=6.8 \mathrm{~Hz}, 2 \mathrm{H}), 1.78-1.76(\mathrm{~m}, 4 \mathrm{H}) ;{ }^{13} \mathrm{C} \mathrm{NMR}\left(100 \mathrm{MHz}, \mathrm{CDCl}_{3}\right) \delta 170.6,159.08$ $(\mathrm{d}, \mathrm{J}=242.0 \mathrm{~Hz}), 138.7,134.0(\mathrm{~d}, \mathrm{~J}=3.0 \mathrm{~Hz}), 133.8,129.3,127.7,121.70(\mathrm{~d}, \mathrm{~J}=8.0$ $\mathrm{Hz}), 115.29(\mathrm{~d}, \mathrm{~J}=22.0 \mathrm{~Hz}), 55.7,36.1,23.9$, 22.0; HRMS (ESI): Calcd for $\mathrm{C}_{17} \mathrm{H}_{19} \mathrm{FNO}_{3} \mathrm{~S}[\mathrm{M}+\mathrm{H}]:$ 336.1064, found: 336.1063 .<smiles>O=C(CCCC[SeH](=O)(O)c1ccccc1)Nc1ccc(Br)cc1</smiles>

\section{$\mathrm{N}$-(4-Bromophenyl)-5-(phenylsulfonyl)pentanamide (3e)}

White solid (Reaction time: $6 \mathrm{~h}$, FCC eluent: EA/PE = 1:2, $192 \mathrm{mg}$, yield: 97\%), mp: 137-139 ${ }^{\circ} \mathrm{C}$; IR (KBr) v 3325, 1769, 1664, 1244, 1148, 813, $737 \mathrm{~cm}^{-1} ;{ }^{1} \mathrm{H}$ NMR (400 $\left.\mathrm{MHz} \mathrm{CDCl}_{3}\right) \delta 8.08(\mathrm{~s}, 1 \mathrm{H}), 7.88-7.86(\mathrm{~m}, 2 \mathrm{H}), 7.65(\mathrm{t}, \mathrm{J}=7.2 \mathrm{~Hz}, 1 \mathrm{H}), 7.55(\mathrm{t}, \mathrm{J}=$ $7.6 \mathrm{~Hz}, 2 \mathrm{H}), 7.37$ (q, J = 8.8 Hz, 4H), 3.14-3.11 (m, 2H), $2.34(\mathrm{t}, \mathrm{J}=6.8 \mathrm{~Hz}, 2 \mathrm{H})$, $1.78(\mathrm{p}, \mathrm{J}=3.6 \mathrm{~Hz}, 4 \mathrm{H}) ;{ }^{13} \mathrm{C} \mathrm{NMR}\left(100 \mathrm{MHz}, \mathrm{CDCl}_{3}\right) \delta 170.6,138.7,137.0,133.9$, 131.7, 129.4, 127.8, 127.7, 121.4, 116.6, 55.7, 36.3, 23.8, 22.0; HRMS (ESI): Calcd for $\mathrm{C}_{17} \mathrm{H}_{19} \mathrm{NBrO}_{3} \mathrm{~S}[\mathrm{M}+\mathrm{H}]:$ 396.0264, found: 396.0269 .<smiles>N#Cc1ccc(NC(=O)CCCCS(=O)(=O)c2ccccc2)cc1</smiles>

\section{$N$-(4-Cyanophenyl)-5-(phenylsulfonyl)pentanamide (3f)}

White solid (Reaction time: $8 \mathrm{~h}$, FCC eluent: EA/PE = 1:1, $140 \mathrm{mg}$, yield: 82\%), mp: 158-159 ${ }^{\circ} \mathrm{C}$; IR (KBr) v 2225, 1297, 1249, 842, $722 \mathrm{~cm}^{-1} ;{ }^{1} \mathrm{H}$ NMR (400 MHz, DMSO) $\delta 10.30(\mathrm{~s}, 1 \mathrm{H}), 7.90(\mathrm{~d}, J=8.0 \mathrm{~Hz}, 2 \mathrm{H}), 7.75-7.72(\mathrm{~m}, 5 \mathrm{H}), 7.67-7.63(\mathrm{~m}$, $2 \mathrm{H}), 3.36(\mathrm{t}, J=7.2 \mathrm{~Hz}, 2 \mathrm{H}), 2.37-2.36(\mathrm{~m}, 2 \mathrm{H}), 1.67-1.61(\mathrm{~m}, 4 \mathrm{H}) ;{ }^{13} \mathrm{C} \mathrm{NMR}(100$ MHz, DMSO) $\delta 171.5,143.3,139.0,133.7,133.2$, 129.4, 127.6, 119.1, 119.0, 104.7, 54.3, 35.7, 23.3, 21.8; HRMS (ESI): Calcd for $\mathrm{C}_{18} \mathrm{H}_{19} \mathrm{~N}_{2} \mathrm{O}_{3} \mathrm{~S}$ [M+H]: 343.1111, found: 343.1114 .<smiles>Cc1ccccc1NC(=O)C(C)CCCS(=O)(=O)c1ccccc1</smiles>

\section{2-Methyl-5-(phenylsulfonyl)- $\boldsymbol{N}$-(o-tolyl)pentanamide (3g)}

White solid (Reaction time: $8 \mathrm{~h}$, FCC eluent: EA/PE = 1:2, $107 \mathrm{mg}$, yield: 62\%), mp: 144-145 ${ }^{\circ} \mathrm{C}$; IR (KBr) v 1244, 1142, 844, $748 \mathrm{~cm}^{-1} ;{ }^{1} \mathrm{H}$ NMR (400 MHz, $\left.\mathrm{CDCl}_{3}\right) \delta$ $7.86(\mathrm{~d}, J=8.0 \mathrm{~Hz}, 2 \mathrm{H}), 7.62(\mathrm{q}, J=7.2 \mathrm{~Hz}, 2 \mathrm{H}), 7.53(\mathrm{t}, J=7.6 \mathrm{~Hz}, 2 \mathrm{H}), 7.29(\mathrm{~s}$, $1 \mathrm{H}), 7.16-7.14(\mathrm{~m}, 2 \mathrm{H}), 7.06(\mathrm{t}, J=7.2 \mathrm{~Hz}, 1 \mathrm{H}), 3.10(\mathrm{t}, J=7.2 \mathrm{~Hz}, 2 \mathrm{H}), 2.40(\mathrm{p}, J=$ $6.8 \mathrm{~Hz}, 1 \mathrm{H}), 2.20(\mathrm{~s}, 3 \mathrm{H}), 1.83-1.74(\mathrm{~m}, 3 \mathrm{H}), 1.56-150(\mathrm{~m}, 1 \mathrm{H}), 1.21(\mathrm{~d}, J=6.8 \mathrm{~Hz}$, $3 \mathrm{H}) ;{ }^{13} \mathrm{C} \mathrm{NMR}\left(100 \mathrm{MHz}, \mathrm{CDCl}_{3}\right) \delta 174.0,138.9,135.4,133.7,130.4,129.9,129.3$, 127.9, 126.5, 125.4, 123.7, 55.8, 41.4, 32.5, 20.6, 18.0, 17.8; HRMS (ESI): Calcd for $\mathrm{C}_{19} \mathrm{H}_{24} \mathrm{NO}_{3} \mathrm{~S}[\mathrm{M}+\mathrm{H}]: 346.1471$, found: 346.1471 . 
$\mathrm{N}_{\mathrm{O}}^{\mathrm{N}} \mathrm{MSO}_{2} \mathrm{Ph}$

2,2-Dimethyl-5-(phenylsulfonyl)- $\boldsymbol{N}$-(o-tolyl)pentanamide (3h)

White solid (Reaction time: $8 \mathrm{~h}$, FCC eluent: EA/PE = 1:3, $161 \mathrm{mg}$, yield: $90 \%$ ), 92-94 ${ }^{\circ} \mathrm{C}$; IR ( $\left.\mathrm{KBr}\right) \vee 1303,1246,749 \mathrm{~cm}^{-1}$; ${ }^{1} \mathrm{H}$ NMR (400 MHz, $\left.\mathrm{CDCl}_{3}\right) \delta 7.81(\mathrm{~d}, J$ $=8.0 \mathrm{~Hz}, 2 \mathrm{H}), 7.60(\mathrm{t}, J=7.2 \mathrm{~Hz}, 1 \mathrm{H}), 7.55(\mathrm{~d}, J=6.8 \mathrm{~Hz}, 1 \mathrm{H}), 7.49(\mathrm{t}, J=7.6 \mathrm{~Hz}$, $2 \mathrm{H}), 7.39(\mathrm{~s}, 1 \mathrm{H}), 7.14(\mathrm{t}, J=6.6 \mathrm{~Hz}, 2 \mathrm{H}), 7.05(\mathrm{t}, J=7.4 \mathrm{~Hz}, 1 \mathrm{H}), 3.04-3.01(\mathrm{~m}, 2 \mathrm{H})$, $2.16(\mathrm{~s}, 3 \mathrm{H}), 1.73-1.67(\mathrm{~m}, 4 \mathrm{H}), 1.24(\mathrm{~s}, 6 \mathrm{H}) ;{ }^{13} \mathrm{C} \mathrm{NMR}\left(100 \mathrm{MHz}, \mathrm{CDCl}_{3}\right) \delta 175.0$, 138.8, 135.4, 133.5, 130.2, 130.0, 129.1, 127.7, 126.4, 125.2, 123.6, 56.0, 42.7, 39.1, 25.2, 18.3, 17.5; HRMS (ESI): Calcd for $\mathrm{C}_{20} \mathrm{H}_{26} \mathrm{NO}_{3} \mathrm{~S}[\mathrm{M}+\mathrm{H}]$ : 360.1628, found: 360.1627 .<smiles>Cc1ccccc1NC(=O)CC(C)(C)CCOc1ccccc1</smiles>

\section{3,3-Dimethyl-5-(phenylsulfonyl)- $N$-(o-tolyl)pentanamide (3i)}

Colorless oil (Reaction time: 8 h, FCC eluent: EA/PE $=1: 3,177 \mathrm{mg}$, yield: $99 \%$ ), $\mathrm{R}_{\mathrm{f}}$ $=0.30(\mathrm{EA} / \mathrm{PE}=1: 5) ; \mathrm{IR}(\mathrm{KBr}) \vee 1299,1249,781,750 \mathrm{~cm}^{-1} ;{ }^{1} \mathrm{H}$ NMR $(400 \mathrm{MHz}$, $\left.\mathrm{CDCl}_{3}\right) \delta 7.81(\mathrm{~d}, J=8.0 \mathrm{~Hz}, 2 \mathrm{H}), 7.61(\mathrm{t}, J=7.6 \mathrm{~Hz}, 1 \mathrm{H}), 7.53-7.49(\mathrm{~m}, 3 \mathrm{H}), 7.44$ $(\mathrm{d}, J=7.6 \mathrm{~Hz}, 1 \mathrm{H}), 7.10(\mathrm{t}, J=7.6 \mathrm{~Hz}, 2 \mathrm{H}), 7.05(\mathrm{t}, J=7.2 \mathrm{~Hz}, 1 \mathrm{H}), 3.19-3.15$ (m, $2 \mathrm{H}), 2.14(\mathrm{~s}, 5 \mathrm{H}), 1.73-1.67(\mathrm{~m}, 2 \mathrm{H}), 1.24(\mathrm{~s}, 6 \mathrm{H}) ;{ }^{13} \mathrm{C} \mathrm{NMR}\left(100 \mathrm{MHz}, \mathrm{CDCl}_{3}\right) \delta$ 169.2, 138.7, 135.2, 133.6, 130.5, 130.3, 129.1, 127.8, 126.2, 125.4, 124.2, 52.2, 47.8, 34.0, 32.9, 27.0, 17.9; HRMS (ESI): Calcd for $\mathrm{C}_{20} \mathrm{H}_{26} \mathrm{NO}_{3} \mathrm{~S}[\mathrm{M}+\mathrm{H}]: 360.1628$, found: 360.1627 .<smiles>CCN(C(=O)CCCC[SeH]Oc1ccccc1)c1ccccc1</smiles>

\section{$N$-Ethyl- $N$-phenyl-5-(phenylsulfonyl)pentanamide (3j)}

Colorless oil (Reaction time: $6 \mathrm{~h}$, eluent: FCC EA/PE = 1:1, $90 \mathrm{mg}$, yield: $52 \%$ ), $\mathrm{R}_{\mathrm{f}}=$ $0.27(\mathrm{EA} / \mathrm{PE}=1: 2)$; IR (KBr) $v 1299,1252,842,770 \mathrm{~cm}^{-1} ;{ }^{1} \mathrm{H}$ NMR (400 MHz, $\left.\mathrm{CDCl}_{3}\right) \delta 7.86(\mathrm{~d}, J=7.2 \mathrm{~Hz}, 2 \mathrm{H}), 7.65(\mathrm{t}, J=7.6 \mathrm{~Hz}, 1 \mathrm{H}), 7.55(\mathrm{t}, J=7.6 \mathrm{~Hz}, 2 \mathrm{H})$, 7.42 (t, $J=7.6 \mathrm{~Hz}, 2 \mathrm{H}), 7.38-7.34(\mathrm{~m}, 1 \mathrm{H}), 7.11(\mathrm{~d}, J=7.6 \mathrm{~Hz}, 2 \mathrm{H}), 3.71$ (q, $J=7.2$ $\mathrm{Hz}, 2 \mathrm{H}), 3.03-2.98(\mathrm{~m}, 2 \mathrm{H}), 2.00-1.97(\mathrm{~m}, 2 \mathrm{H}), 1.65-1.61(\mathrm{~m}, 4 \mathrm{H}), 1.08(\mathrm{t}, J=7.2$ $\mathrm{Hz}, 3 \mathrm{H}) ;{ }^{13} \mathrm{C} \mathrm{NMR}\left(100 \mathrm{MHz}, \mathrm{CDCl}_{3}\right) \delta 171.2,142.1,139.0,133.5,129.7,129.1$, 128.2, 127.8, 127.9, 55.9, 43.9, 33.5, 23.9, 22.1, 12.9; HRMS (ESI): Calcd for $\mathrm{C}_{19} \mathrm{H}_{24} \mathrm{NO}_{3} \mathrm{~S}[\mathrm{M}+\mathrm{H}]: 346.1471$, found: 346.1479 .<smiles>O=C(CCCC[SH](=O)(O)c1ccccc1)N(Cc1ccccc1)c1ccccc1</smiles>

$N$-Benzyl- $N$-phenyl-5-(phenylsulfonyl)pentanamide (3k) 
Colorless oil (Reaction time: 10 h, FCC eluent: EA/PE $=1: 1,140 \mathrm{mg}$, yield: $69 \%$ ), $\mathrm{R}_{\mathrm{f}}$ $=0.27(\mathrm{EA} / \mathrm{PE}=1: 2) ; \mathrm{IR}(\mathrm{KBr}) \vee 1303,1245,745 \mathrm{~cm}^{-1} ;{ }^{1} \mathrm{H} \mathrm{NMR}\left(400 \mathrm{MHz}, \mathrm{CDCl}_{3}\right)$ $\delta 7.86(\mathrm{~d}, \mathrm{~J}=7.6 \mathrm{~Hz}, 2 \mathrm{H}), 7.63(\mathrm{t}, \mathrm{J}=7.6 \mathrm{~Hz}, 1 \mathrm{H}), 7.53(\mathrm{t}, \mathrm{J}=7.6 \mathrm{~Hz}, 2 \mathrm{H}), 7.30$ (d, J $=5.2 \mathrm{~Hz}, 3 \mathrm{H}), 7.24-7.23(\mathrm{~m}, 3 \mathrm{H}), 7.16-7.14(\mathrm{~m}, 2 \mathrm{H}), 6.92(\mathrm{~d}, J=7.2 \mathrm{~Hz}, 2 \mathrm{H}), 4.84$ (s, 2H), 3.02-3.00 (m, 2H), 2.06-2.03 (m, 2H), 1.68-1.63 (m, 4H); ${ }^{13} \mathrm{C}$ NMR (100 $\left.\mathrm{MHz}, \mathrm{CDCl}_{3}\right) \delta 171.6,141.9,138.9,137.2,133.5,129.5,129.1,128.6,128.2,128.1$, 127.9, 127.8, 127.2, 55.8, 52.8, 33.4, 23.9, 22.1; HRMS (ESI): Calcd for $\mathrm{C}_{24} \mathrm{H}_{26} \mathrm{NO}_{3} \mathrm{~S}$ [M+H]: 406.1628, found: 406.1625 .

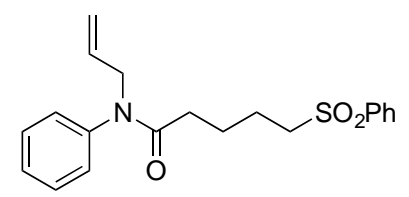

\section{$N$-Allyl- $N$-phenyl-5-(phenylsulfonyl)pentanamide (3l)}

Colorless oil (Reaction time: 24 h, FCC eluent: EA/PE $=1: 1,60 \mathrm{mg}$, yield: $34 \%$ ), $\mathrm{R}_{\mathrm{f}}$ $=0.17(\mathrm{EA} / \mathrm{PE}=1: 2) ; \mathrm{IR}(\mathrm{KBr}) \vee 1303,1244,831,749 \mathrm{~cm}^{-1} ;{ }^{1} \mathrm{H}$ NMR $(400 \mathrm{MHz}$, $\left.\mathrm{CDCl}_{3}\right) \delta 7.87(\mathrm{~d}, \mathrm{~J}=7.6 \mathrm{~Hz}, 2 \mathrm{H}), 7.65(\mathrm{t}, \mathrm{J}=7.6 \mathrm{~Hz}, 1 \mathrm{H}), 7.55(\mathrm{t}, \mathrm{J}=7.2 \mathrm{~Hz}, 2 \mathrm{H})$, $7.40(\mathrm{t}, \mathrm{J}=7.6 \mathrm{~Hz}, 2 \mathrm{H}), 7.36-7.33(\mathrm{~m}, 1 \mathrm{H}), 7.11(\mathrm{~d}, \mathrm{~J}=7.6 \mathrm{~Hz}, 2 \mathrm{H}), 5.87-5.77$ (m, $1 \mathrm{H}), 5.10-5.02(\mathrm{~m}, 2 \mathrm{H}), 4.25(\mathrm{~d}, J=6.0 \mathrm{~Hz}, 2 \mathrm{H}), 3.03-2.99(\mathrm{~m}, 2 \mathrm{H}), 2.05-2.02(\mathrm{~m}$, 2H), 1.65-1.63 (m, 4H); ${ }^{13} \mathrm{C}$ NMR (100 MHz, $\left.\mathrm{CDCl}_{3}\right) \delta 171.4,142.2,139.0,133.6$, 132.9, 129.6, 129.2, 128.2, 128.0, 127.9, 117.8, 55.9, 52.1, 33.5, 23.9, 22.2; HRMS (ESI): Calcd for $\mathrm{C}_{20} \mathrm{H}_{24} \mathrm{NO}_{3} \mathrm{~S}[\mathrm{M}+\mathrm{H}]: 358.1471$, found 358.1479.

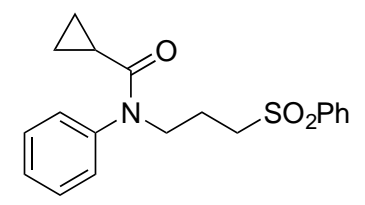

\section{$N$-Phenyl- $N$-(3-(phenylsulfonyl)propyl)cyclopropanecarboxamide (3m)}

Colorless oil (Reaction time: 9 h, FCC eluent: EA/PE = 1:1, $141 \mathrm{mg}$, yield: $82 \%$ ), $\mathrm{R}_{\mathrm{f}}$ $=0.27(\mathrm{EA} / \mathrm{PE}=1: 2) ; \mathrm{IR}(\mathrm{KBr}) \vee 1245,1141,700 \mathrm{~cm}^{-1} ;{ }^{1} \mathrm{H} \mathrm{NMR}\left(400 \mathrm{MHz}, \mathrm{CDCl}_{3}\right)$ $\delta 7.85(\mathrm{~d}, J=6.4 \mathrm{~Hz}, 2 \mathrm{H}), 7.67-7.62(\mathrm{~m}, 1 \mathrm{H}), 7.56-7.53(\mathrm{~m}, 2 \mathrm{H}), 7.43-7.39(\mathrm{~m}, 2 \mathrm{H})$, 7.36-7.32 (m, 1H), 7.17 (d, $J=7.2 \mathrm{~Hz}, 2 \mathrm{H}), 3.80(\mathrm{~d}, J=5.6 \mathrm{~Hz}, 2 \mathrm{H}), 3.17-3.13(\mathrm{~m}$, $2 \mathrm{H}), 1.94-1.88(\mathrm{~m}, 2 \mathrm{H}), 1.28-1.23(\mathrm{~m}, 1 \mathrm{H}), 0.94(\mathrm{t}, J=4.0 \mathrm{~Hz}, 2 \mathrm{H}), 0.60-0.57(\mathrm{~m}$, $2 \mathrm{H}) ;{ }^{13} \mathrm{C} \mathrm{NMR}\left(100 \mathrm{MHz}, \mathrm{CDCl}_{3}\right) \delta 173.5,141.7,138.6,133.5,129.6,129.1,128.0$, 127.8, 127.7, 53.6, 47.0, 21.2, 12.4, 8.4; HRMS (ESI): Calcd for $\mathrm{C}_{19} \mathrm{H}_{22} \mathrm{NO}_{3} \mathrm{~S}[\mathrm{M}+\mathrm{H}]$ : 344.1315, found: 344.1322 .

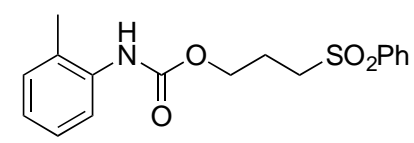

\section{3-(Phenylsulfonyl)propyl $o$-tolylcarbamate (3n)}

White solid (Reaction time: 10 h, FCC eluent: EA/PE = 1:3, $127 \mathrm{mg}$, yield: 76\%), mp: 97-99 ${ }^{\circ} \mathrm{C}$; IR ( $\left.\mathrm{KBr}\right)$ v 1230, 1145, $755 \mathrm{~cm}^{-1}$; ${ }^{1} \mathrm{H}$ NMR (400 MHz, $\left.\mathrm{CDCl}_{3}\right) \delta 7.91-7.89$ 
(m, 2H), $7.65(\mathrm{t}, J=7.6 \mathrm{~Hz}, 2 \mathrm{H}), 7.56(\mathrm{~d}, J=7.6 \mathrm{~Hz}, 2 \mathrm{H}), 7.18-7.13(\mathrm{~m}, 2 \mathrm{H}), 7.04-$ $7.00(\mathrm{~m}, 1 \mathrm{H}), 6.54(\mathrm{~s}, 1 \mathrm{H}), 4.21-4.18(\mathrm{~m}, 2 \mathrm{H}), 3.23-3.19(\mathrm{~m}, 2 \mathrm{H}), 2.22(\mathrm{~s}, 3 \mathrm{H}), 2.12-$ $2.05(\mathrm{~m}, 2 \mathrm{H}) ;{ }^{13} \mathrm{C} \mathrm{NMR}\left(100 \mathrm{MHz}, \mathrm{CDCl}_{3}\right) \delta 153.4,138.6,135.4,133.8,130.3,129.3$, 127.9, 126.6, 124.4, 62.6, 53.0, 22.6, 17.5; HRMS (ESI): Calcd for $\mathrm{C}_{17} \mathrm{H}_{19} \mathrm{NO}_{4} \mathrm{SNa}$ [M+Na]: 356.0927, found: 356.0935.

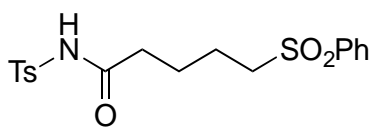

\section{5-(Phenylsulfonyl)- $N$-tosylpentanamide (3o)}

White solid (Reaction time: 4.5 h, FCC eluent: EA/PE = 1:1, $151 \mathrm{mg}$, yield: 76\%), mp: 161-163 ${ }^{\circ} \mathrm{C}$; IR $(\mathrm{KBr}) \vee 1286,1246,1144,846,751 \mathrm{~cm}^{-1} ;{ }^{1} \mathrm{H}$ NMR (400 MHz, DMSO) $\delta 12.01(\mathrm{~s}, 1 \mathrm{H}), 7.88(\mathrm{~d}, J=8.0 \mathrm{~Hz}, 2 \mathrm{H}), 7.81(\mathrm{~d}, J=7.6 \mathrm{~Hz}, 2 \mathrm{H}), 7.76(\mathrm{t}, J=$ $7.6 \mathrm{~Hz}, 1 \mathrm{H}), 7.66$ (t, $J=7.6 \mathrm{~Hz}, 2 \mathrm{H}), 7.42(\mathrm{~d}, J=8.0 \mathrm{~Hz}, 2 \mathrm{H}), 3.28(\mathrm{~d}, J=7.2 \mathrm{~Hz}$, 2H), $2.40(\mathrm{~s}, 3 \mathrm{H}), 2.22(\mathrm{~d}, J=6.4 \mathrm{~Hz}, 2 \mathrm{H}), 1.48(\mathrm{~d}, J=5.6 \mathrm{~Hz}, 4 \mathrm{H}) ;{ }^{13} \mathrm{C}$ NMR $(100$ MHz, DMSO) $\delta 171.1,144.2,139.0,136.6,133.8,129.5,129.4,127.6,127.5,54.2$, 34.7, 22.5, 21.6, 21.1; HRMS (ESI): Calcd for $\mathrm{C}_{18} \mathrm{H}_{22} \mathrm{NO}_{5} \mathrm{~S}_{2}[\mathrm{M}+\mathrm{H}]: 396.0934$, found: 396.0934 .

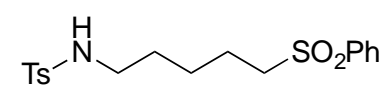

\section{4-Methyl- $N$-(5-(phenylsulfonyl)pentyl)benzenesulfonamide (3p)}

White solid (Reaction time: 4.5 h, FCC eluent: EA/PE = 1:1, $166 \mathrm{mg}$, yield: 87\%), mp: $100-101{ }^{\circ} \mathrm{C}$; IR $(\mathrm{KBr}) \vee 1305,1244,1155,816,729 \mathrm{~cm}^{-1} ;{ }^{1} \mathrm{H}$ NMR $(400 \mathrm{MHz}$, $\left.\mathrm{CDCl}_{3}\right) \delta 7.87(\mathrm{~d}, J=7.6 \mathrm{~Hz}, 2 \mathrm{H}), 7.72(\mathrm{~d}, J=8.0 \mathrm{~Hz}, 2 \mathrm{H}), 7.65(\mathrm{t}, J=7.6 \mathrm{~Hz}, 1 \mathrm{H})$, $7.56(\mathrm{t}, J=7.6 \mathrm{~Hz}, 2 \mathrm{H}), 7.28(\mathrm{~d}, J=8.0 \mathrm{~Hz}, 2 \mathrm{H}), 5.20-5.17(\mathrm{~m}, 1 \mathrm{H}), 3.04-3.00(\mathrm{~m}$, $2 \mathrm{H}), 2.85(\mathrm{q}, J=6.4 \mathrm{~Hz}, 2 \mathrm{H}), 2.41(\mathrm{~s}, 3 \mathrm{H}), 1.67-1.60(\mathrm{~m}, 2 \mathrm{H}), 1.46-1.38(\mathrm{~m}, 2 \mathrm{H})$, 1.37-1.31 (m, 2H); ${ }^{13} \mathrm{C}$ NMR $\left(100 \mathrm{MHz}, \mathrm{CDCl}_{3}\right) \delta 143.2,138.8,136.7,133.6,129.6$, 129.2, 127.8, 126.9, 55.7, 42.5, 28.8, 25.0, 22.0, 21.4; HRMS (ESI): Calcd for $\mathrm{C}_{18} \mathrm{H}_{24} \mathrm{NO}_{4} \mathrm{~S}_{2}[\mathrm{M}+\mathrm{H}]:$ 382.1141, found: 382.1142 .

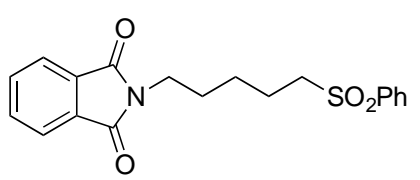

\section{2-(5-(Phenylsulfonyl)pentyl)isoindoline-1,3-dione (3q)}

White solid (Reaction time: $6 \mathrm{~h}$, FCC eluent: EA/PE $=1: 2,143 \mathrm{mg}$, yield: 80\%), mp: $118-120{ }^{\circ} \mathrm{C}$; IR (KBr) v 1243, 1148, $748 \mathrm{~cm}^{-1} ;{ }^{1} \mathrm{H}$ NMR $\left(400 \mathrm{MHz}, \mathrm{CDCl}_{3}\right) \delta 7.90(\mathrm{~d}$, $J=7.6 \mathrm{~Hz}, 2 \mathrm{H}), 7.84-7.80(\mathrm{~m}, 2 \mathrm{H}), 7.72-7.70(\mathrm{~m}, 2 \mathrm{H}), 7.65(\mathrm{t}, J=7.6 \mathrm{~Hz}, 1 \mathrm{H}), 7.56$ $(\mathrm{t}, J=7.6 \mathrm{~Hz}, 2 \mathrm{H}), 3.64(\mathrm{t}, J=7.2 \mathrm{~Hz}, 2 \mathrm{H}), 3.11-3.07(\mathrm{~m}, 2 \mathrm{H}), 1.76(\mathrm{t}, J=8.0 \mathrm{~Hz}$, $2 \mathrm{H}), 1.70-1.62(\mathrm{~m}, 2 \mathrm{H}), 1.42(\mathrm{p}, J=8.0 \mathrm{~Hz}, 2 \mathrm{H}) ;{ }^{13} \mathrm{C} \mathrm{NMR}\left(100 \mathrm{MHz}, \mathrm{CDCl}_{3}\right) \delta$ 168.1, 138.9, 133.8, 133.5, 131.8, 129.1, 127.8, 123.0, 55.8, 37.3, 27.9, 25.4, 22.2; HRMS (ESI): Calcd for $\mathrm{C}_{19} \mathrm{H}_{20} \mathrm{NO}_{4} \mathrm{~S}[\mathrm{M}+\mathrm{H}]$ : 358.1108 , found: 358.1108 . 


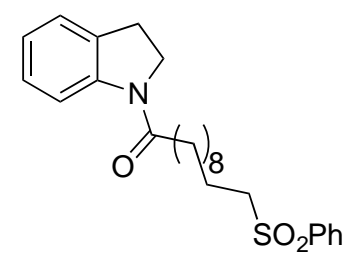

\section{1-(Indolin-1-yl)-11-(phenylsulfonyl)undecan-1-one (3r)}

White solid (Reaction time: 24 h, FCC eluent: EA/PE = 1:1, $190 \mathrm{mg}$, yield: 89\%), mp: 241-243 ${ }^{\circ} \mathrm{C}$; IR (KBr) v 1306, 1146, $754 \mathrm{~cm}^{-1}$; ${ }^{1} \mathrm{H}$ NMR $\left(400 \mathrm{MHz}, \mathrm{CDCl}_{3}\right) \delta 8.23(\mathrm{~d}$, $J=8.0 \mathrm{~Hz}, 1 \mathrm{H}), 7.90-7.88(\mathrm{~m}, 2 \mathrm{H}), 7.65-7.62(\mathrm{~m}, 1 \mathrm{H}), 7.55(\mathrm{t}, J=8.0 \mathrm{~Hz}, 2 \mathrm{H}), 7.15$ $(\mathrm{d}, J=8.0 \mathrm{~Hz}, 2 \mathrm{H}), 6.97(\mathrm{t}, J=7.6 \mathrm{~Hz}, 1 \mathrm{H}), 4.00-3.96(\mathrm{~m}, 2 \mathrm{H}), 3.14(\mathrm{t}, J=8.4 \mathrm{~Hz}$, 2H), 3.08-3.04 (m, 2H), $2.36(\mathrm{t}, J=7.6 \mathrm{~Hz}, 2 \mathrm{H}), 1.72-1.65(\mathrm{~m}, 4 \mathrm{H}), 1.35-1.23(\mathrm{~m}$, $14 \mathrm{H}) ;{ }^{13} \mathrm{C}$ NMR (100 MHz, $\left.\mathrm{CDCl}_{3}\right) \delta 171.2,142.9,138.9,133.4,130.9,129.0,127.7$, 127.1, 124.3, 123.1, 116.6, 56.0, 47.7, 35.6, 29.2, 29.1, 29.0, 28.9, 28.7, 27.9, 27.7, 24.2, 22.3; HRMS (ESI): Calcd for $\mathrm{C}_{25} \mathrm{H}_{34} \mathrm{NO}_{3} \mathrm{~S}$ [M+H]: 428.2254, found: 428.2262 .<smiles>O=C(OCCCCOc1ccccc1)c1ccco1</smiles>

\section{4-(Phenylsulfonyl)butyl furan-2-carboxylate (3s)}

Colorless oil (Reaction time: $12 \mathrm{~h}$, FCC eluent: EA/PE $=1: 2,129 \mathrm{mg}$, yield: $84 \%$ ), $\mathrm{R}_{\mathrm{f}}$ $=0.33(\mathrm{EA} / \mathrm{PE}=1: 2), \mathrm{IR}(\mathrm{KBr}) \vee 1299,1234,1180,1145,1121,763 \mathrm{~cm}^{-1} ;{ }^{1} \mathrm{H}$ NMR (400 MHz, CDCl3) $\delta 7.91(\mathrm{~d}, \mathrm{~J}=7.2 \mathrm{~Hz}, 2 \mathrm{H}), 7.67-7.64(\mathrm{~m}, 1 \mathrm{H}), 7.60-7.56(\mathrm{~m}, 3 \mathrm{H})$, $7.13(\mathrm{t}, J=3.2 \mathrm{~Hz}, 1 \mathrm{H}), 6.52-6.50(\mathrm{~m}, 1 \mathrm{H}), 4.27(\mathrm{~d}, J=5.2 \mathrm{~Hz}, 2 \mathrm{H}), 3.19$ (t, $J=5.6$ $\mathrm{Hz}, 2 \mathrm{H}), 1.87-1.84(\mathrm{~m}, 4 \mathrm{H}) ;{ }^{13} \mathrm{C} \mathrm{NMR}\left(100 \mathrm{MHz}, \mathrm{CDCl}_{3}\right) \delta 158.3,146.2,144.2$, 138.7, 133.5, 129.1, 127.8, 117.9, 111.7, 63.5, 55.4, 27.0, 19.4; HRMS (ESI): Calcd for $\mathrm{C}_{15} \mathrm{H}_{16} \mathrm{NaO}_{5}[\mathrm{M}+\mathrm{Na}]$ : 331.0611, found: 331.0618 .

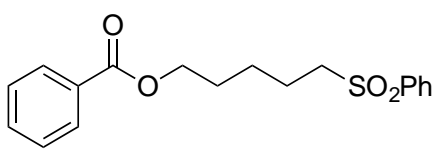

\section{5-(Phenylsulfonyl)pentyl benzoate (3t)}

Colorless oil (Reaction time: $12 \mathrm{~h}$, FCC eluent: EA/PE = 1:2, $144 \mathrm{mg}$, yield: 87\%), $\mathrm{R}_{\mathrm{f}}$ $=0.43(\mathrm{EA} / \mathrm{PE}=1: 2), \mathrm{IR}(\mathrm{KBr}) \vee 1277,1146,1116,714 \mathrm{~cm}^{-1} ;{ }^{1} \mathrm{H}$ NMR $(400 \mathrm{MHz}$, $\left.\mathrm{CDCl}_{3}\right) \delta 8.01(\mathrm{~d}, \mathrm{~J}=7.2 \mathrm{~Hz}, 2 \mathrm{H}), 7.90(\mathrm{~d}, \mathrm{~J}=7.6 \mathrm{~Hz}, 2 \mathrm{H}), 7.64-7.61(\mathrm{~m}, 1 \mathrm{H}), 7.56-$ $7.53(\mathrm{~m}, 3 \mathrm{H}), 7.45-7.42(\mathrm{~m}, 2 \mathrm{H}), 4.29-4.27$ (m, 2H), 3.14-3.10 (m, 2H), 1.80-1.74 $(\mathrm{m}, 4 \mathrm{H}), 1.53(\mathrm{t}, J=7.2 \mathrm{~Hz}, 2 \mathrm{H}) ;{ }^{13} \mathrm{C} \mathrm{NMR}\left(100 \mathrm{MHz}, \mathrm{CDCl}_{3}\right) \delta 166.3,138.9,133.5$, 132.8, 130.0, 129.3, 129.1, 128.2, 127.8, 64.2, 55.9, 28.0, 24.7, 22.3; HRMS (ESI): Calcd for $\mathrm{C}_{18} \mathrm{H}_{20} \mathrm{NaO}_{4} \mathrm{~S}$ [M+Na]: 355.0975, found: 335.0983 .

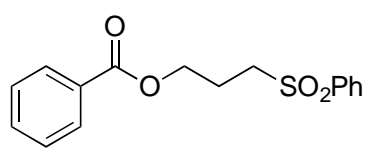

\section{3-(Phenylsulfonyl)propyl benzoate (3u)}


White solid (Reaction time: 10 h, FCC eluent: EA/PE = 1:2, $148 \mathrm{mg}$, yield: 97\%), mp: 77-79 ${ }^{\circ} \mathrm{C}$; IR (KBr) v 1274, 1144, $713 \mathrm{~cm}^{-1} ;{ }^{1} \mathrm{H}$ NMR (400 MHz, $\left.\mathrm{CDCl}_{3}\right) \delta 8.00-7.93$ $(\mathrm{m}, 4 \mathrm{H}), 7.67(\mathrm{t}, J=7.6 \mathrm{~Hz}, 1 \mathrm{H}), 7.60-7.58(\mathrm{~m}, 3 \mathrm{H}), 7.44(\mathrm{t}, J=7.6 \mathrm{~Hz}, 2 \mathrm{H}), 4.38(\mathrm{t}, J$ $=6.5 \mathrm{~Hz}, 2 \mathrm{H}), 3.29-3.25(\mathrm{~m}, 2 \mathrm{H}), 2.25-2.18(\mathrm{~m}, 2 \mathrm{H}) ;{ }^{13} \mathrm{C} \mathrm{NMR}\left(100 \mathrm{MHz}, \mathrm{CDCl}_{3}\right) \delta$ 166.1, 138.8, 133.9, 133.2, 129.6, 129.5, 129.4, 128.4, 128.0, 62.5, 53.2, 22.6; HRMS (ESI): Calcd for $\mathrm{C}_{16} \mathrm{H}_{16} \mathrm{NaO}_{4} \mathrm{~S}$ [M+Na]: 327.0662, found: 327.0667 .<smiles>Cn1cccc1C(=O)OCCCC[SeH](=O)(O)c1ccccc1</smiles>

\section{4-(Phenylsulfonyl)butyl 1-methyl-1H-pyrrole-2-carboxylate (3v)}

Colorless oil (Reaction time: 26 h, FCC eluent: EA/PE = 1:2, $155 \mathrm{mg}$, yield: 97\%), $\mathrm{R}_{\mathrm{f}}$ $=0.40(\mathrm{EA} / \mathrm{PE}=1: 2), \mathrm{IR}(\mathrm{KBr}) \vee 1245,1145,744 \mathrm{~cm}^{-1} ;{ }^{1} \mathrm{H} \mathrm{NMR}(400 \mathrm{MHz}, \mathrm{CDCl} 3)$ $\delta$ 7.91-7.89 (m, 2H), $7.65(\mathrm{t}, \mathrm{J}=7.2 \mathrm{~Hz}, 1 \mathrm{H}), 7.55(\mathrm{t}, \mathrm{J}=7.6 \mathrm{~Hz}, 2 \mathrm{H}), 6.85-6.83(\mathrm{~m}$, $1 \mathrm{H}), 6.78(\mathrm{t}, \mathrm{J}=2.4 \mathrm{~Hz}, 1 \mathrm{H}), 6.09(\mathrm{dd}, \mathrm{J}=4.0,2.5 \mathrm{~Hz}, 1 \mathrm{H}), 4.19(\mathrm{~d}, J=6.0 \mathrm{~Hz}, 2 \mathrm{H})$, $3.88(\mathrm{~s}, 3 \mathrm{H}), 3.18-3.15(\mathrm{~m}, 2 \mathrm{H}), 1.87-1.80(\mathrm{~m}, 4 \mathrm{H}) ;{ }^{13} \mathrm{C} \mathrm{NMR}\left(100 \mathrm{MHz}, \mathrm{CDCl}_{3}\right) \delta$ 160.9, 138.9, 133.6, 129.6, 129.2, 127.9, 122.0, 117.8, 107.8, 62.4, 55.6, 36.6, 27.3, 19.6; HRMS (ESI): Calcd for $\mathrm{C}_{16} \mathrm{H}_{19} \mathrm{NaNO}_{4} \mathrm{~S}$ [M+Na]: 344.0927, found: 344.0934 .

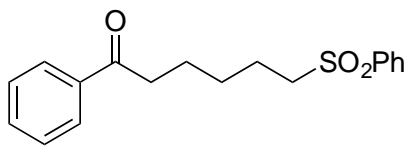

\section{1-Phenyl-6-(phenylsulfonyl)hexan-1-one (3w)}

White solid (Reaction time: $10 \mathrm{~h}$, FCC eluent: EA/PE = 1:3, $135 \mathrm{mg}$, yield: 85\%), mp: $116-117{ }^{\circ} \mathrm{C}$; IR (KBr) v 1301, 1244, 1145, $758 \mathrm{~cm}^{-1} ;{ }^{1} \mathrm{H}$ NMR (400 MHz, $\left.\mathrm{CDCl}_{3}\right) \delta$ $7.93-7.89$ (m, 4H), 7.65 (t, $J=7.2 \mathrm{~Hz}, 1 \mathrm{H}), 7.58-7.53(\mathrm{~m}, 3 \mathrm{H}), 7.45$ (t, $J=7.2 \mathrm{~Hz}$, 2H), 3.13-3.09 (m, 2H), $2.94(\mathrm{t}, J=7.2 \mathrm{~Hz}, 2 \mathrm{H}), 1.81-1.68(\mathrm{~m}, 4 \mathrm{H}), 1.46$ (p, $J=7.6$ $\mathrm{Hz}, 2 \mathrm{H}) ;{ }^{13} \mathrm{C}$ NMR $\left(100 \mathrm{MHz}, \mathrm{CDCl}_{3}\right) \delta 199.6,136.8,133.6,133.0,129.2,128.5$, 127.9, 127.8, 56.0, 37.8, 27.8, 23.3, 22.5; HRMS (ESI): Calcd for $\mathrm{C}_{18} \mathrm{H}_{21} \mathrm{O}_{3} \mathrm{~S}[\mathrm{M}+\mathrm{H}]$ : 317.1206, found: 317.1205 .<smiles>[3H]OCCCCCCS(=O)(=O)c1ccccc1</smiles>

\section{5-(Phenylsulfonyl)pentyl 4-methylbenzenesulfonate (3x)}

Colorless oil (Reaction time: $8 \mathrm{~h}$, FCC eluent: EA/PE = 1:1, $122 \mathrm{mg}$, yield: $64 \%$ ), $\mathrm{R}_{\mathrm{f}}$ $=0.33(\mathrm{EA} / \mathrm{PE}=1: 2), \mathrm{IR}(\mathrm{KBr}) v 1303,1244,1176,1146,816,749 \mathrm{~cm}^{-1} ;{ }^{1} \mathrm{H}$ NMR $\left(400 \mathrm{MHz}, \mathrm{CDCl}_{3}\right) \delta$ 7.89-7.87 (m, 2H), $7.75(\mathrm{~d}, J=8.4 \mathrm{~Hz}, 2 \mathrm{H}), 7.68-7.64(\mathrm{~m}, 1 \mathrm{H})$, $7.57(\mathrm{t}, J=7.2 \mathrm{~Hz}, 2 \mathrm{H}), 7.34(\mathrm{~d}, J=8.0 \mathrm{~Hz}, 2 \mathrm{H}), 3.98(\mathrm{t}, J=6.0 \mathrm{~Hz}, 2 \mathrm{H}), 3.06-3.02$ $(\mathrm{m}, 2 \mathrm{H}), 2.44(\mathrm{~s}, 3 \mathrm{H}), 1.72-1.60(\mathrm{~m}, 4 \mathrm{H}), 1.44-1.37(\mathrm{~m}, 2 \mathrm{H}) ;{ }^{13} \mathrm{C}$ NMR $(100 \mathrm{MHz}$, $\left.\mathrm{CDCl}_{3}\right) \delta 144.8,138.8,133.6,132.7,129.8,129.2,127.8,127.6,69.7,55.7,28.2,24.1$, 21.9, 21.5; HRMS (ESI): Calcd. for $\mathrm{C}_{18} \mathrm{H}_{22} \mathrm{O}_{5} \mathrm{~S}_{2} \mathrm{Na}$ [M+Na]: 405.0801, found: 405.0808 . 
$\mathrm{NOSO}_{2} \mathrm{Ph}$

\section{((4-Phenoxybutyl)sulfonyl)benzene (3y)}

White solid (Reaction time: 10 h, FCC eluent: EA/PE = 1:5, $122 \mathrm{mg}$, yield: 84\%), mp: $103-105{ }^{\circ} \mathrm{C}$; IR $(\mathrm{KBr}) \vee 1305,1243,1144,754 \mathrm{~cm}^{-1} ;{ }^{1} \mathrm{H}$ NMR $\left(400 \mathrm{MHz}, \mathrm{CDCl}_{3}\right) \delta$ 7.91-7.89 (m, 2H), $7.64(\mathrm{t}, J=7.6 \mathrm{~Hz}, 1 \mathrm{H}), 7.57-7.53(\mathrm{~m}, 2 \mathrm{H}), 7.27-7.23(\mathrm{~m}, 2 \mathrm{H})$, $6.92(\mathrm{t}, J=7.2 \mathrm{~Hz}, 1 \mathrm{H}), 6.82(\mathrm{~d}, J=8.0 \mathrm{~Hz}, 2 \mathrm{H}), 3.93-3.90(\mathrm{~m}, 2 \mathrm{H}), 3.19-3.15(\mathrm{~m}$, 2H), 1.94-1.83 (m, 4H); ${ }^{13} \mathrm{C}$ NMR (100 MHz, $\left.\mathrm{CDCl}_{3}\right) \delta 158.5,138.9,133.6,129.4$, 129.3, 129.2, 127.9, 120.7, 114.3, 66.6, 55.8, 27.7, 19.8; HRMS (ESI): Calcd for $\mathrm{C}_{16} \mathrm{H}_{19} \mathrm{O}_{3} \mathrm{~S}[\mathrm{M}+\mathrm{H}]: 291.1049$, found: 291.1052 .<smiles>O=C(O)CCCCS(=O)(=O)c1ccccc1</smiles>

\section{5-(Phenylsulfonyl)pentanoic acid (3z)}

Colorless oil (Reaction time: 14 h, FCC eluent: EA/PE = 1:1, $106 \mathrm{mg}$, yield: $88 \%$ ), $\mathrm{R}_{\mathrm{f}}$ $=0.2(\mathrm{EA} / \mathrm{PE}=1: 1), \mathrm{IR}(\mathrm{KBr}) \vee 1290,1243,1147,732 \mathrm{~cm}^{-1} ;{ }^{1} \mathrm{H}$ NMR $(400 \mathrm{MHz}$, $\left.\mathrm{CDCl}_{3}\right) \delta 11.03(\mathrm{~s}, 1 \mathrm{H}), 7.92-7.90(\mathrm{~m}, 2 \mathrm{H}), 7.67(\mathrm{t}, J=7.6 \mathrm{~Hz}, 1 \mathrm{H}), 7.58(\mathrm{t}, J=7.6$ $\mathrm{Hz}, 2 \mathrm{H}), 3.14-3.10(\mathrm{~m}, 2 \mathrm{H}), 2.35(\mathrm{t}, J=7.2 \mathrm{~Hz}, 2 \mathrm{H}), 1.83-1.68(\mathrm{~m}, 4 \mathrm{H}) ;{ }^{13} \mathrm{C} \mathrm{NMR}$ $\left(100 \mathrm{MHz}, \mathrm{CDCl}_{3}\right) \delta 178.6,138.9,133.7,129.3,128.0,55.7,33.2,23.1,22.0$; HRMS (ESI): Calcd for $\mathrm{C}_{11} \mathrm{H}_{14} \mathrm{O}_{4} \mathrm{SNa}$ [M+Na]: 265.0505, found: 265.0506 .<smiles>O=C(O)CCCCCCSc1ccccc1</smiles>

\section{7-(Phenylsulfonyl)heptanoic acid (3aa)}

White solid (Reaction time: 14 h, FCC eluent: EA/PE = 1:1, $134 \mathrm{mg}$, yield: 99\%), mp: 117-118 ${ }^{\circ} \mathrm{C}$; IR (KBr) v 1243, 1145, 798, $746 \mathrm{~cm}^{-1} ;{ }^{1} \mathrm{H}$ NMR (400 MHz, $\left.\mathrm{CDCl}_{3}\right) \delta$ 10.88 (s, 1H), 7.92-7. 90 (m, 2H), $7.67(\mathrm{t}, J=7.2 \mathrm{~Hz}, 1 \mathrm{H}), 7.58(\mathrm{t}, J=7.6 \mathrm{~Hz}, 2 \mathrm{H})$, $3.12-3.08(\mathrm{~m}, 2 \mathrm{H}), 2.32(\mathrm{t}, J=7.2 \mathrm{~Hz}, 2 \mathrm{H}), 1.76-1.68(\mathrm{~m}, 2 \mathrm{H}), 1.59$ (p, $J=7.2 \mathrm{~Hz}$, 2H), 1.43-1.30 (m, 4H); ${ }^{13} \mathrm{C}$ NMR (100 MHz, $\left.\mathrm{CDCl}_{3}\right) \delta 179.5,138.9,133.6,129.2$, 127.9, 56.0, 33.62, 28.2, 27.7, 24.0, 22.3; HRMS (ESI): Calcd for $\mathrm{C}_{13} \mathrm{H}_{18} \mathrm{O}_{4} \mathrm{SNa}$ [M+Na]: 293.0818, found: 293.0817.<smiles>O=S(=O)(CCCCCCO)c1ccccc1</smiles>

\section{6-(Phenylsulfonyl)hexan-1-ol (3ab)}

Colorless oil (Reaction time: 20 h, FCC eluent: EA/PE $=1: 1,83 \mathrm{mg}$, yield: $69 \%$ ), $\mathrm{R}_{\mathrm{f}}$ $=0.4(\mathrm{EA} / \mathrm{PE}=1: 1), \mathrm{IR}(\mathrm{KBr}) \vee 1302,1244,1147,745 \mathrm{~cm}^{-1} ;{ }^{1} \mathrm{H}$ NMR $(400 \mathrm{MHz}$, $\left.\mathrm{CDCl}_{3}\right) \delta 7.90(\mathrm{~d}, J=7.2 \mathrm{~Hz}, 2 \mathrm{H}), 7.66(\mathrm{t}, J=7.2 \mathrm{~Hz}, 1 \mathrm{H}), 7.57(\mathrm{t}, J=7.2 \mathrm{~Hz}, 2 \mathrm{H})$, 3.59 (t, $J=6.4 \mathrm{~Hz}, 2 \mathrm{H}), 3.11-3.08(\mathrm{~m}, 2 \mathrm{H}), 2.09(\mathrm{~s}, 1 \mathrm{H}), 1.76-1.70(\mathrm{~m}, 2 \mathrm{H}), 1.51$ (p, $J=6.8 \mathrm{~Hz}, 2 \mathrm{H}), 1.42-1.31(\mathrm{~m}, 4 \mathrm{H}) ;{ }^{13} \mathrm{C} \mathrm{NMR}\left(100 \mathrm{MHz}, \mathrm{CDCl}_{3}\right) \delta 139.0,133.6$, 129.2, 127.9, 62.3, 56.0, 32.1, 27.9, 25.1, 22.5; HRMS (ESI): Calcd for $\mathrm{C}_{12} \mathrm{H}_{19} \mathrm{O}_{3} \mathrm{~S}$ [M+H]: 243.1049, found: 243.1051 .

$\mathrm{PhO}_{2} \mathrm{~S} \sim \mathrm{Br}_{\mathrm{Br}}$ 
((5-Bromopentyl)sulfonyl)benzene (3ac)

Colorless oil (Reaction time: 24 h, FCC eluent: EA/PE = 1:2, $141 \mathrm{mg}$, yield: $97 \%$ ), $\mathrm{R}_{\mathrm{f}}$ $=0.67(\mathrm{EA} / \mathrm{PE}=1: 1), \mathrm{IR}(\mathrm{KBr}) \vee 1304,1237,1147,891,790 \mathrm{~cm}^{-1} ;{ }^{1} \mathrm{H}$ NMR $(400$ $\left.\mathrm{MHz} \mathrm{CDCl}_{3}\right) \delta 7.90(\mathrm{~d}, \mathrm{~J}=8.0 \mathrm{~Hz}, 2 \mathrm{H}), 7.67(\mathrm{t}, J=7.2 \mathrm{~Hz}, 1 \mathrm{H}), 7.58(\mathrm{t}, J=7.6 \mathrm{~Hz}$, $2 \mathrm{H}), 3.37-3.34(\mathrm{~m}, 2 \mathrm{H}), 3.13-3.09(\mathrm{~m}, 2 \mathrm{H}), 1.82(\mathrm{q}, J=7.2 \mathrm{~Hz}, 2 \mathrm{H}), 1.73(\mathrm{q}, J=8.0$ $\mathrm{Hz}, 2 \mathrm{H}), 1.56-1.48(\mathrm{~m}, 2 \mathrm{H}) ;{ }^{13} \mathrm{C}$ NMR $\left(100 \mathrm{MHz}, \mathrm{CDCl}_{3}\right) \delta 138.8,133.5,129.1$, 127.7, 55.7, 32.9, 31.8, 26.5, 21.7; HRMS (ESI): Calcd for $\mathrm{C}_{11} \mathrm{H}_{16} \mathrm{O}_{2} \mathrm{BrS}[\mathrm{M}+\mathrm{H}]$ : 291.0049, found: 291.0055 .

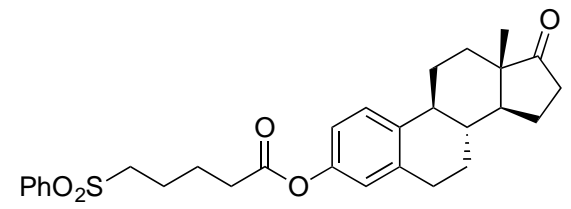

$(8 R, 9 S, 13 S, 14 S)-13-M e t h y l-17-0 x 0-7,8,9,11,12,13,14,15,16,17-d e c a h y d r o-6 H$-cycl openta[a]phenanthren-3-yl 5-(phenylsulfonyl)pentanoate (3ad)

White solid (Reaction time: 24 h, FCC eluent: EA/PE = 1:1, $240 \mathrm{mg}$, yield: 97\%), mp: $151-153{ }^{\circ} \mathrm{C}$; IR (KBr) v 1305, 1146, $720 \mathrm{~cm}^{-1}$; ${ }^{1} \mathrm{H}$ NMR $\left(400 \mathrm{MHz}, \mathrm{CDCl}_{3}\right) \delta 7.92-$ $7.90(\mathrm{~m}, 2 \mathrm{H}), 7.66(\mathrm{t}, J=7.6 \mathrm{~Hz}, 1 \mathrm{H}), 7.56(\mathrm{t}, J=7.6 \mathrm{~Hz}, 2 \mathrm{H}), 7.29-7.25(\mathrm{~m}, 1 \mathrm{H})$, 6.81-6.77 (m, 2H), $3.15(\mathrm{t}, J=7.2 \mathrm{~Hz}, 2 \mathrm{H}), 2.90-2.87(\mathrm{~m}, 2 \mathrm{H}), 2.54(\mathrm{t}, J=6.4 \mathrm{~Hz}$, $2 \mathrm{H}), 2.50-2.45(\mathrm{~m}, 1 \mathrm{H}), 2.40-2.37(\mathrm{~m}, 1 \mathrm{H}), 2.28-2.23(\mathrm{~m}, 4 \mathrm{H}), 2.17-1.93(\mathrm{~m}, 4 \mathrm{H})$, $1.84-1.82(\mathrm{~m}, 4 \mathrm{H}), 1.64-1.43(\mathrm{~m}, 6 \mathrm{H}), 0.89(\mathrm{~s}, 3 \mathrm{H}) ;{ }^{13} \mathrm{C} \mathrm{NMR}\left(100 \mathrm{MHz}, \mathrm{CDCl}_{3}\right) \delta$ 171.3, 148.2, 138.8, 137.8, 137.2, 133.5, 129.1, 127.8, 126.1, 121.2, 118.4, 55.5, 50.1, 47.6, 43.8, 37.7, 35.6, 33.4, 31.3, 29.1, 26.0, 25.5, 23.3, 22.0, 21.3, 13.6; HRMS (ESI): Calcd for $\mathrm{C}_{29} \mathrm{H}_{35} \mathrm{O}_{4} \mathrm{~S}[\mathrm{M}+\mathrm{H}]:$ 495.2200, found: 495.2208 .<smiles>CC(C)CCS(=O)(=O)c1ccccc1</smiles>

\section{(Dodecylsulfonyl)benzene (3ae)}

Colorless oil (Reaction time: 6 h, FCC eluent: EA/PE $=1: 5,152 \mathrm{mg}$, yield: $98 \%$ ), $\mathrm{R}_{\mathrm{f}}$ $=0.6(\mathrm{EA} / \mathrm{PE}=1: 2), \mathrm{IR}(\mathrm{KBr}) \vee 3065,2854,1306,1243,790 \mathrm{~cm}^{-1} ;{ }^{1} \mathrm{H}$ NMR $(400$ $\left.\mathrm{MHz} \mathrm{CDCl}_{3}\right) \delta 7.91(\mathrm{~d}, \mathrm{~J}=7.6 \mathrm{~Hz}, 2 \mathrm{H}), 7.67(\mathrm{t}, \mathrm{J}=7.6 \mathrm{~Hz}, 1 \mathrm{H}), 7.56(\mathrm{t}, \mathrm{J}=7.6 \mathrm{~Hz}$, 2H), 3.10-3.06 (m, 2H), 1.74-1.67 (m, 2H), 1.36-1.32 (m, 2H), 1.29-1.23 (m, 16H), $0.88(\mathrm{t}, J=7.2 \mathrm{~Hz}, 3 \mathrm{H}) ;{ }^{13} \mathrm{C} \mathrm{NMR}\left(100 \mathrm{MHz}, \mathrm{CDCl}_{3}\right) \delta 139.2,133.5,129.1,127.9$, 56.2, 31.8, 29.5, 29.4, 29.3, 29.2, 29.1, 28.9, 28.1, 22.6, 22.5, 14.0; HRMS (ESI): Calcd for $\mathrm{C}_{18} \mathrm{H}_{30} \mathrm{O}_{2} \mathrm{SNa}$ [M+Na]: 333.1859, found: 333.1855 .

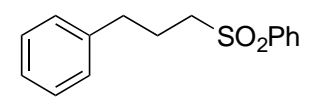

\section{((3-Phenylpropyl)sulfonyl)benzene (3af)}

White solid (Reaction time: $10 \mathrm{~h}$, FCC eluent: EA/PE = 1:3, $90 \mathrm{mg}$, yield: 69\%), mp: 93-95 ${ }^{\circ} \mathrm{C}$; IR (KBr) v 1305, 1248, 1148, 780, $747 \mathrm{~cm}^{-1} ;{ }^{1} \mathrm{H}$ NMR $\left(400 \mathrm{MHz}, \mathrm{CDCl}_{3}\right)$ $\delta 7.88-7.86(\mathrm{~m}, 2 \mathrm{H}), 7.63(\mathrm{t}, J=7.6 \mathrm{~Hz}, 1 \mathrm{H}), 7.54(\mathrm{t}, J=7.6 \mathrm{~Hz}, 2 \mathrm{H}), 7.27-7.24(\mathrm{~m}$, 
2H), $7.18(\mathrm{t}, J=7.2 \mathrm{~Hz}, 1 \mathrm{H}), 7.09(\mathrm{~d}, J=7.2 \mathrm{~Hz}, 2 \mathrm{H}), 3.08-3.05(\mathrm{~m}, 2 \mathrm{H}), 2.69(\mathrm{t}, J=$ $7.6 \mathrm{~Hz}, 2 \mathrm{H}), 2.06-2.02(\mathrm{~m}, 2 \mathrm{H}) ;{ }^{13} \mathrm{C} \mathrm{NMR}\left(100 \mathrm{MHz}, \mathrm{CDCl}_{3}\right) \delta 139.8,139.1,133.6$, 129.2, 128.5, 128.3, 127.9, 126.3, 55.3, 34.0, 24.1; HRMS (ESI): Calcd for $\mathrm{C}_{15} \mathrm{H}_{16} \mathrm{O}_{2} \mathrm{SNa}[\mathrm{M}+\mathrm{Na}]: 283.0763$, found: 283.0761.<smiles>O=S(=O)(CCCCc1ccccc1)c1ccccc1</smiles>

\section{((4-Phenylbutyl)sulfonyl)benzene (3ag)}

White solid (Reaction time: 10 h, FCC eluent: EA/PE = 1:3, $131 \mathrm{mg}$, yield: 96\%), mp: 63-65 ${ }^{\circ} \mathrm{C}$; IR (KBr) v 1304, 1244, 1148, $744 \mathrm{~cm}^{-1} ;{ }^{1} \mathrm{H}$ NMR $\left(400 \mathrm{MHz}, \mathrm{CDCl}_{3}\right) \delta$ $7.89-7.86(\mathrm{~m}, 2 \mathrm{H}), 7.64(\mathrm{t}, J=7.6 \mathrm{~Hz}, 1 \mathrm{H}), 7.54(\mathrm{t}, J=8.0 \mathrm{~Hz}, 2 \mathrm{H}), 7.24(\mathrm{t}, J=7.2$ $\mathrm{Hz}, 2 \mathrm{H}), 7.16$ (d, $J=7.2 \mathrm{~Hz}, 1 \mathrm{H}), 7.09$ (d, $J=7.2 \mathrm{~Hz}, 2 \mathrm{H}), 3.11-3.07$ (m, 2H), 2.59$2.56(\mathrm{~m}, 2 \mathrm{H}), 1.77-1.66(\mathrm{~m}, 4 \mathrm{H}) ;{ }^{13} \mathrm{C} \mathrm{NMR}\left(100 \mathrm{MHz}, \mathrm{CDCl}_{3}\right) \delta 141.1,139.1,133.6$, 129.2, 128.3, 128.2, 127.9, 125.9, 56.0, 35.2, 29.9, 22.2; HRMS (ESI): Calcd for $\mathrm{C}_{16} \mathrm{H}_{18} \mathrm{O}_{2} \mathrm{SNa}[\mathrm{M}+\mathrm{Na}]: 297.0920$, found: 297.0916.

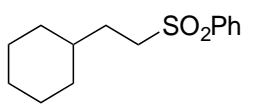

\section{((2-Cyclohexylethyl)sulfonyl)benzene (3ah)}

Colorless oil (Reaction time: 10 h, FCC eluent: EA/PE = 1:3, $124 \mathrm{mg}$, yield: $98 \%$ ), $\mathrm{R}_{\mathrm{f}}$ $=0.43(\mathrm{EA} / \mathrm{PE}=1: 2), \mathrm{IR}(\mathrm{KBr}) \vee 3064,2852,1299,1246,1153,844,736 \mathrm{~cm}^{-1} ;{ }^{1} \mathrm{H}$ NMR (400 MHz, $\left.\mathrm{CDCl}_{3}\right) \delta 7.92-7.90(\mathrm{~m}, 2 \mathrm{H}), 7.66(\mathrm{t}, \mathrm{J}=7.2 \mathrm{~Hz}, 1 \mathrm{H}), 7.57(\mathrm{t}, \mathrm{J}=$ $7.2 \mathrm{~Hz}, 2 \mathrm{H}), 3.10(\mathrm{t}, \mathrm{J}=8.4 \mathrm{~Hz}, 2 \mathrm{H}), 1.68-1.61(\mathrm{~m}, 7 \mathrm{H}), 1.16-1.13(\mathrm{~m}, 4 \mathrm{H}), 0.88$ $0.84(\mathrm{~m}, 2 \mathrm{H}) ;{ }^{13} \mathrm{C}$ NMR $\left(100 \mathrm{MHz}, \mathrm{CDCl}_{3}\right) \delta 139.1,133.5,129.1,127.9,54.2,36.4$, 32.6, 29.5, 26.1, 25.8; HRMS (ESI): Calcd for $\mathrm{C}_{14} \mathrm{H}_{21} \mathrm{O}_{2} \mathrm{~S}$ [M+H]: 253.1257, found: 253.1257 .<smiles>CC(CCCC(=O)Nc1ccccc1)Oc1ccccc1</smiles>

\section{$N$-Phenyl-5-(phenylsulfonyl)hexanamide (3ai-1)}

Colorless oil (Reaction time: 10 h, FCC eluent: EA/PE $=1: 2,140 \mathrm{mg}$, yield: $85 \%$ ), $\mathrm{R}_{\mathrm{f}}$ $=0.5(\mathrm{EA} / \mathrm{PE}=1: 1), \mathrm{IR}(\mathrm{KBr}) \vee 1302,1246,1143,847,733 \mathrm{~cm}^{-1} ;{ }^{1} \mathrm{H}$ NMR $(400$ $\left.\mathrm{MHz}, \mathrm{CDCl}_{3}\right) \delta 8.05(\mathrm{~s}, 1 \mathrm{H}), 7.85-7.73(\mathrm{~m}, 2 \mathrm{H}), 7.62(\mathrm{t}, J=7.2 \mathrm{~Hz}, 1 \mathrm{H}), 7.54-7.49$ (m, 4H), 7.28-7.24 (m, 2H), $7.06(\mathrm{t}, J=7.2 \mathrm{~Hz}, 1 \mathrm{H}), 3.09-3.05(\mathrm{~m}, 1 \mathrm{H}), 2.37-2.32$ (m, 2H), 2.06-2.00 (m, 1H), 1.93-1.85 (m, 1H), 1.72-1.64 (m, 1H), 1.55-1.47 (m, $1 \mathrm{H}), 1.23(\mathrm{~d}, J=7.2 \mathrm{~Hz}, 3 \mathrm{H}) ;{ }^{13} \mathrm{C} \mathrm{NMR}\left(100 \mathrm{MHz}, \mathrm{CDCl}_{3}\right) \delta 170.7,138.0,136.8$, 133.7, 129.1, 128.8, 124.0, 119.9, 59.7, 36.7, 28.4, 22.4, 13.4; HRMS (ESI): Calcd for $\mathrm{C}_{18} \mathrm{H}_{22} \mathrm{NO}_{3} \mathrm{~S}[\mathrm{M}+\mathrm{H}]: 332.1315$, found: 332.1313 .<smiles>CCC(CCC(=O)Nc1ccccc1)S(=O)(=O)O</smiles> 


\section{$N$-Phenyl-4-(phenylsulfonyl)hexanamide (3ai-2)}

Colorless oil (Reaction time: 10 h, FCC eluent: EA/PE = 1:2, $22 \mathrm{mg}$, yield: 13\%), $\mathrm{R}_{\mathrm{f}}$ $=0.3(\mathrm{EA} / \mathrm{PE}=1: 2)$; IR $(\mathrm{KBr}) \vee 1301,1247,1143,756 \mathrm{~cm}^{-1} ;{ }^{1} \mathrm{H}$ NMR $(400 \mathrm{MHz}$, $\left.\mathrm{CDCl}_{3}\right) \delta 7.89-7.86(\mathrm{~m}, 2 \mathrm{H}), 7.66(\mathrm{t}, J=7.2 \mathrm{~Hz}, 2 \mathrm{H}), 7.58-7.52(\mathrm{~m}, 4 \mathrm{H}), 7.32(\mathrm{t}, J=$ $7.6 \mathrm{~Hz}, 2 \mathrm{H}), 7.11(\mathrm{t}, J=7.6 \mathrm{~Hz}, 1 \mathrm{H}), 3.08-3.01(\mathrm{~m}, 1 \mathrm{H}), 2.86-2.79(\mathrm{~m}, 1 \mathrm{H}), 2.66-$ $2.61(\mathrm{~m}, 1 \mathrm{H}), 2.29-2.13(\mathrm{~m}, 2 \mathrm{H}), 1.80-1.78(\mathrm{~m}, 1 \mathrm{H}), 1.54-1.45(\mathrm{~m}, 1 \mathrm{H}), 0.96(\mathrm{t}, J=$ $7.2 \mathrm{~Hz}, 3 \mathrm{H}) ;{ }^{13} \mathrm{C} \mathrm{NMR}\left(100 \mathrm{MHz}, \mathrm{CDCl}_{3}\right) \delta 170.2,137.8,137.6,133.8,129.2,129.0$, 128.7, 124.3, 119.8, 64.7, 34.3, 22.9, 22.0, 11.0; HRMS (ESI): Calcd for $\mathrm{C}_{18} \mathrm{H}_{21} \mathrm{NO}_{3} \mathrm{SNa}[\mathrm{M}+\mathrm{Na}]: 354.1134$, found: 354.1136 .

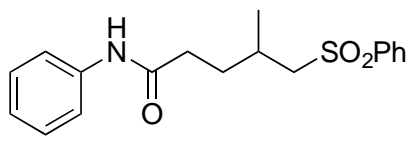

\section{4-Methyl- $N$-phenyl-5-(phenylsulfonyl)pentanamide (3aj)}

White solid (Reaction time: 14 h, FCC eluent: EA/PE = 1:2, $160 \mathrm{mg}$, yield: 97\%), $126-128{ }^{\circ} \mathrm{C}$; IR (KBr) v 1298, 1249, 841, $752 \mathrm{~cm}^{-1} ;{ }^{1} \mathrm{H}$ NMR (400 MHz, $\left.\mathrm{CDCl}_{3}\right) \delta$ $8.33(\mathrm{~s}, 1 \mathrm{H}), 7.87-7.85(\mathrm{~m}, 2 \mathrm{H}), 7.59(\mathrm{t}, J=7.2 \mathrm{~Hz}, 1 \mathrm{H}), 7.53-7.47(\mathrm{~m}, 4 \mathrm{H}), 7.24(\mathrm{t}, J$ $=8.0 \mathrm{~Hz}, 2 \mathrm{H}), 7.05(\mathrm{t}, J=7.2 \mathrm{~Hz}, 1 \mathrm{H}), 3.14-3.09(\mathrm{~m}, 1 \mathrm{H}), 2.97-2.92(\mathrm{~m}, 1 \mathrm{H}), 2.40$ $2.32(\mathrm{~m}, 2 \mathrm{H}), 2.16-2.10(\mathrm{~m}, 1 \mathrm{H}), 1.87-1.79(\mathrm{~m}, 1 \mathrm{H}), 1.75-1.67(\mathrm{~m}, 1 \mathrm{H}), 1.02(\mathrm{~d}, J=$ $6.8 \mathrm{~Hz}, 3 \mathrm{H}) ;{ }^{13} \mathrm{C} \mathrm{NMR}\left(100 \mathrm{MHz}, \mathrm{CDCl}_{3}\right) \delta 171.0,139.4,138.0,133.6,129.2,128.6$, 127.5, 123.9, 119.8, 61.7, 34.2, 32.0, 28.0, 19.8; HRMS (ESI): Calcd for $\mathrm{C}_{18} \mathrm{H}_{22} \mathrm{NO}_{3} \mathrm{~S}$ $[\mathrm{M}+\mathrm{H}]: 332.1315$, found: 332.1315 .

$\mathrm{PhO}_{2} \mathrm{~S}-$

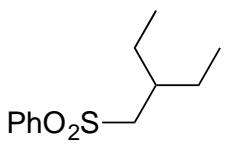

\section{((2-Ethylbutyl)sulfonyl)benzene (3ak) ${ }^{11}$}

White solid (Reaction time: $8 \mathrm{~h}$, FCC eluent: EA/PE = 1:10, $76 \mathrm{mg}$, yield: 67\%), mp: $70-72{ }^{\circ} \mathrm{C}$; IR $(\mathrm{KBr})$ v 1290, 1178, 1146, 1126, 883, $748 \mathrm{~cm}^{-1} ;{ }^{1} \mathrm{H}$ NMR (400 MHz, $\left.\mathrm{CDCl}_{3}\right) \delta 7.92(\mathrm{~d}, J=7.2 \mathrm{~Hz}, 2 \mathrm{H}), 7.65(\mathrm{~d}, J=7.2 \mathrm{~Hz}, 1 \mathrm{H}), 7.58-7.55(\mathrm{~m}, 2 \mathrm{H}), 3.02$ $(\mathrm{d}, J=6.0 \mathrm{~Hz}, 2 \mathrm{H}), 1.92-1.86(\mathrm{~m}, 1 \mathrm{H}), 1.49-1.44(\mathrm{~m}, 4 \mathrm{H}), 0.81(\mathrm{t}, J=7.6 \mathrm{~Hz}, 6 \mathrm{H})$; ${ }^{13} \mathrm{C}$ NMR $\left(100 \mathrm{MHz}, \mathrm{CDCl}_{3}\right) \delta 140.0,133.4,129.1,127.7,59.4,35.62,25.1,10.1$.<smiles>CC(C)C(C)(C)S(=O)(=O)c1ccccc1</smiles>

\section{((2,3-Dimethylbutan-2-yl)sulfonyl)benzene (3al) ${ }^{12}$}

Colorless oil (Reaction time: $8 \mathrm{~h}, \mathrm{FCC}$ eluent: EA/PE $=1: 10,110 \mathrm{mg}$, yield: $97 \%$ ), $\mathrm{R}_{\mathrm{f}}$ $=0.59(\mathrm{EA} / \mathrm{PE}=1: 5), \mathrm{IR}(\mathrm{KBr}) \vee 1305,1149,748 \mathrm{~cm}^{-1} ;{ }^{1} \mathrm{H} \mathrm{NMR}\left(400 \mathrm{MHz}, \mathrm{CDCl}_{3}\right)$ $\delta 7.89-7.86(\mathrm{~m}, 2 \mathrm{H}), 7.64(\mathrm{t}, J=7.6 \mathrm{~Hz}, 1 \mathrm{H}), 7.55(\mathrm{t}, J=7.6 \mathrm{~Hz}, 2 \mathrm{H}), 2.21(\mathrm{p}, J=6.8$ $\mathrm{Hz}, 1 \mathrm{H}), 1.24(\mathrm{~s}, 6 \mathrm{H}), 1.08(\mathrm{~d}, J=6.8 \mathrm{~Hz}, 6 \mathrm{H}) ;{ }^{13} \mathrm{C} \mathrm{NMR}\left(100 \mathrm{MHz}, \mathrm{CDCl}_{3}\right) \delta 136.7$, $133.3,130.3,128.6,66.4,31.5,19.4,18.9$. 
$\mathrm{MeO}-\mathrm{SO}^{\mathrm{SO}_{2} \mathrm{Ph}}$

Methyl (1S,2R)-2-(phenylsulfonyl)cyclopentane-1-carboxylate (3am)

White solid (Reaction time: 29 h, FCC eluent: EA/PE = 1:3, $66 \mathrm{mg}$, yield: 49\%), mp: 96-98 ${ }^{\circ} \mathrm{C}$; IR (KBr) v 3229, 2950, 1298, 1245, 1135, 841, $752 \mathrm{~cm}^{-1} ;{ }^{1} \mathrm{H}$ NMR (400 $\left.\mathrm{MHz} \mathrm{CDCl}_{3}\right) \delta 7.92-7.89(\mathrm{~m}, 2 \mathrm{H}), 7.65(\mathrm{t}, J=7.2 \mathrm{~Hz}, 1 \mathrm{H}), 7.58-7.54(\mathrm{~m}, 2 \mathrm{H}), 4.03-$ $3.97(\mathrm{~m}, 1 \mathrm{H}), 3.51(\mathrm{~s}, 3 \mathrm{H}), 3.28-3.22(\mathrm{~m}, 1 \mathrm{H}), 2.23-2.18(\mathrm{~m}, 2 \mathrm{H}), 2.05-2.03(\mathrm{~m}, 1 \mathrm{H})$, $1.82-1.79(\mathrm{~m}, 3 \mathrm{H}),{ }^{13} \mathrm{C} \mathrm{NMR}\left(100 \mathrm{MHz}, \mathrm{CDCl}_{3}\right) \delta 173.9,138.3,133.7,129.1,128.6$, 66.2, 52.2, 45.2, 32.0, 27.3, 25.7; HRMS (ESI): Calcd for $\mathrm{C}_{13} \mathrm{H}_{17} \mathrm{O}_{4} \mathrm{~S}[\mathrm{M}+\mathrm{H}]$ : 269.0842, found: 269.0843 .

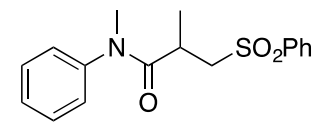

\section{N,2-Dimethyl-N-phenyl-3-(phenylsulfonyl)propanamide (3an)}

White solid (Reaction time: $26 \mathrm{~h}$, FCC eluent: EA/PE = 1:1, $79 \mathrm{mg}$, yield: 50\%), mp: 98-100 ${ }^{\circ} \mathrm{C}$; IR (KBr) v 3463, 1307, 1146, 877, $746 \mathrm{~cm}^{-1} ;{ }^{1} \mathrm{H}$ NMR (400 MHz, $\mathrm{CDCl}_{3}$ ), $\delta 7.83(\mathrm{~d}, J=7.2 \mathrm{~Hz}, 2 \mathrm{H}), 7.63(\mathrm{t}, J=7.2 \mathrm{~Hz}, 1 \mathrm{H}), 7.51(\mathrm{t}, J=7.2 \mathrm{~Hz}, 2 \mathrm{H}), 7.45(\mathrm{t}, J$ $=7.2 \mathrm{~Hz}, 2 \mathrm{H}), 7.38(\mathrm{t}, J=7.2 \mathrm{~Hz}, 1 \mathrm{H}), 7.30(\mathrm{~d}, J=7.6 \mathrm{~Hz}, 2 \mathrm{H}), 3.88(\mathrm{dd}, J=14.0$, $8.8 \mathrm{~Hz}, 1 \mathrm{H}), 3.25$ (s, 3H), 3.11-3.07 (m, 1H), 2.92 (dd, $J=14.0,3.6 \mathrm{~Hz}, 1 \mathrm{H}), 1.11$ (d, $J=7.2 \mathrm{~Hz}, 3 \mathrm{H}) ;{ }^{13} \mathrm{C} \mathrm{NMR}\left(100 \mathrm{MHz}, \mathrm{CDCl}_{3}\right) \delta 173.1,143.2,139.6,133.5,129.8$, 129.1, 128.1, 127.7, 127.4, 59.3, 37.7, 31.3, 18.6; HRMS (ESI): Calcd for $\mathrm{C}_{17} \mathrm{H}_{20} \mathrm{NO}_{3} \mathrm{~S}[\mathrm{M}+\mathrm{H}]: 318.1158$, found: 318.1146 .<smiles>CC(=O)OCCS(=O)(=O)c1ccccc1</smiles>

\section{2-(Phenylsulfonyl)ethyl acetate (3ap)}

Colorless oil (Reaction time: 12 h, FCC eluent: EA/PE = 1:2, $84 \mathrm{mg}$, yield: $74 \%$ ), $\mathrm{R}_{\mathrm{f}}$ $=0.39(\mathrm{EA} / \mathrm{PE}=1: 2) ; \mathrm{IR}(\mathrm{KBr}) \vee 3538,1734,1234,809,767 \mathrm{~cm}^{-1} ;{ }^{1} \mathrm{H}$ NMR $(400$ $\left.\mathrm{MHz}, \mathrm{CDCl}_{3}\right) \delta 7.93(\mathrm{~d}, J=7.6 \mathrm{~Hz}, 2 \mathrm{H}), 7.68(\mathrm{~d}, J=7.6 \mathrm{~Hz}, 1 \mathrm{H}), 7.59$ (t, $J=7.6 \mathrm{~Hz}$, $2 \mathrm{H}), 4.41(\mathrm{t}, J=6.0 \mathrm{~Hz}, 2 \mathrm{H}), 3.47(\mathrm{t}, J=6.0 \mathrm{~Hz}, 2 \mathrm{H}), 1.82(\mathrm{~s}, 3 \mathrm{H}) ;{ }^{13} \mathrm{C}$ NMR $(100$ $\left.\mathrm{MHz}, \mathrm{CDCl}_{3}\right) \delta 170.1,139.4,133.9,129.3,128.0,57.6,54.9,20.3$; HRMS (ESI): Calcd for $\mathrm{C}_{10} \mathrm{H}_{12} \mathrm{O}_{4} \mathrm{SNa}$ [M+Na]: 251.0349, found: 251.0349 .

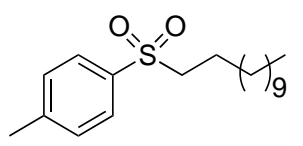

\section{1-(Dodecylsulfonyl)-4-methylbenzene (4a)}

White solid (Reaction time: $6 \mathrm{~h}$, FCC eluent: EA/PE $=1: 2,160 \mathrm{mg}$, yield: 99\%), mp: 67-69 ${ }^{\circ} \mathrm{C}$; IR ( $\left.\mathrm{KBr}\right) \vee 1285,1245,1145,820,762 \mathrm{~cm}^{-1} ;{ }^{1} \mathrm{H}$ NMR $\left(400 \mathrm{MHz}, \mathrm{CDCl}_{3}\right)$ $\delta 7.78(\mathrm{~d}, J=8.4 \mathrm{~Hz}, 2 \mathrm{H}), 7.35(\mathrm{~d}, J=7.2 \mathrm{~Hz}, 2 \mathrm{H}), 3.08-3.04(\mathrm{~m}, 2 \mathrm{H}), 2.45(\mathrm{~s}, 3 \mathrm{H})$, $1.73-1.65$ (m, 2H), 1.33 (d, $J=7.6 \mathrm{~Hz}, 2 \mathrm{H}), 1.29-1.22$ (m, 16H), 0.89-0.86 (m, 3H); 
${ }^{13} \mathrm{C} \mathrm{NMR}\left(100 \mathrm{MHz}, \mathrm{CDCl}_{3}\right) \delta 144.4,136.2,129.7,128.0,56.3,31.8,29.5,29.4,29.3$, 29.2, 29.1, 28.9, 28.2, 22.6, 22.5, 21.5, 14.0; HRMS (ESI): Calcd for $\mathrm{C}_{19} \mathrm{H}_{33} \mathrm{O}_{2} \mathrm{~S}$ $[\mathrm{M}+\mathrm{H}]: 325.2196$, found: 325.2198 .<smiles>COc1ccc(S(=O)(=O)CCC(C)(C)C)cc1</smiles>

\section{1-(Dodecylsulfonyl)-4-methoxybenzene (4b)}

White solid (Reaction time: 7 h, FCC eluent: EA/PE = 1:2, $78 \mathrm{mg}$, yield: 46\%), 61-63 ${ }^{\circ} \mathrm{C}$; IR (KBr) v 3439, 1292, 1146, 1026, 836, $763 \mathrm{~cm}^{-1} ;{ }^{1} \mathrm{H}$ NMR (400 MHz, $\left.\mathrm{CDCl}_{3}\right) \delta$ $7.83(\mathrm{~d}, J=8.8 \mathrm{~Hz}, 2 \mathrm{H}), 7.02(\mathrm{~d}, J=8.8 \mathrm{~Hz}, 2 \mathrm{H}), 3.89$ (s, 3H), 3.07-3.03 (m, 2H), $1.69-1.66(\mathrm{~m}, 2 \mathrm{H}), 1.36-1.22(\mathrm{~m}, 18 \mathrm{H}), 0.88(\mathrm{t}, J=6.4 \mathrm{~Hz}, 3 \mathrm{H}) ;{ }^{13} \mathrm{C}$ NMR $(100 \mathrm{MHz}$, $\left.\mathrm{CDCl}_{3}\right) \delta 163.6,130.7,130.1,114.3,56.6,55.6,31.8,29.6,29.5,29.4,29.3,29.2$, 29.0, 28.2, 22.8, 22.6, 14.1. HRMS (ESI): Calcd for $\mathrm{C}_{19} \mathrm{H}_{32} \mathrm{NaO}_{3} \mathrm{~S}$ [M+Na]: 363.1960 , found: 363.1960 .<smiles>CC(C)(C)CCS(=O)(=O)c1ccc(F)cc1</smiles>

\section{1-(Dodecylsulfonyl)-4-fluorobenzene (4c)}

White solid (Reaction time: $5 \mathrm{~h}$, FCC eluent: EA/PE = 1:2, $163 \mathrm{mg}$, yield: 99\%), 58-60 ${ }^{\circ} \mathrm{C}$; IR (KBr) v 1284, 1242, 1149, 843, $772 \mathrm{~cm}^{-1} ;{ }^{1} \mathrm{H}$ NMR (400 MHz, $\left.\mathrm{CDCl}_{3}\right)$ $\delta 7.93(\mathrm{dd}, J=8.8,4.8 \mathrm{~Hz}, 2 \mathrm{H}), 7.25(\mathrm{t}, J=8.4 \mathrm{~Hz}, 2 \mathrm{H}), 3.10-3.06(\mathrm{~m}, 2 \mathrm{H}), 1.72-$ $1.66(\mathrm{~m}, 2 \mathrm{H}), 1.37-1.33(\mathrm{~m}, 2 \mathrm{H}), 1.24-1.23(\mathrm{~m}, 16 \mathrm{H}), 0.88(\mathrm{t}, J=6.4 \mathrm{~Hz}, 3 \mathrm{H}) ;{ }^{13} \mathrm{C}$ NMR (100 MHz, $\left.\mathrm{CDCl}_{3}\right) \delta 165.7(\mathrm{~d}, J=254.0 \mathrm{~Hz}), 135.3(\mathrm{~d}, J=3.0 \mathrm{~Hz}), 130.86$ (d, $J=9.0 \mathrm{~Hz}), 116.48(\mathrm{~d}, J=23.0 \mathrm{~Hz}), 56.4,31.8,29.5,29.4,29.3,29.2,29.1,28.9,28.2$, 22.6, 22.5, 14.0; HRMS (ESI): Calcd for $\mathrm{C}_{18} \mathrm{H}_{30} \mathrm{FO}_{2} \mathrm{~S}[\mathrm{M}+\mathrm{H}]$ : 329.1945, found: 329.1947.<smiles>CC(C)CCS(=O)(=O)c1ccc(Cl)cc1</smiles>

\section{1-Chloro-4-(dodecylsulfonyl)benzene (4d)}

White solid (Reaction time: 12 h, FCC eluent: EA/PE = 1:2, $170 \mathrm{mg}$, yield: 99\%), mp: 146-148 ${ }^{\circ} \mathrm{C}$; IR (KBr) v 1275, 1152, $831 \mathrm{~cm}^{-1} ;{ }^{1} \mathrm{H}$ NMR (400 MHz, $\left.\mathrm{CDCl}_{3}\right) \delta 7.85(\mathrm{~d}$, $J=8.4 \mathrm{~Hz}, 2 \mathrm{H}), 7.54(\mathrm{~d}, J=8.2 \mathrm{~Hz}, 2 \mathrm{H}), 3.10-3.06(\mathrm{~m}, 2 \mathrm{H}), 1.73-1.66(\mathrm{~m}, 2 \mathrm{H})$, $1.37-1.33(\mathrm{~m}, 2 \mathrm{H}), 1.25-1.23(\mathrm{~m}, 16 \mathrm{H}), 0.87(\mathrm{t}, J=6.4 \mathrm{~Hz}, 3 \mathrm{H}) ;{ }^{13} \mathrm{C}$ NMR $(100 \mathrm{MHz}$, $\left.\mathrm{CDCl}_{3}\right) \delta 140.2$, 137.6, 129.5, 129.4, 56.2, 31.8, 29.5, 29.4, 29.3, 29.2, 29.1, 28.8, 28.1, 22.5, 14.0; HRMS (ESI): Calcd for $\mathrm{C}_{18} \mathrm{H}_{30} \mathrm{ClO}_{2} \mathrm{~S}[\mathrm{M}+\mathrm{H}]$ : 345.1650, found: 345.1656 .<smiles>CC(C)CCS(=O)(=O)c1ccc(Br)cc1</smiles> 


\section{1-Bromo-4-(dodecylsulfonyl)benzene (4e)}

White solid (Reaction time: 12 h, FCC eluent: EA/PE = 1:2, $187 \mathrm{mg}$, yield: 96\%), mp: 59-61 ${ }^{\circ} \mathrm{C}$; IR ( $\left.\mathrm{KBr}\right) \vee 1271,1243,1151,828,775 \mathrm{~cm}^{-1} ;{ }^{1} \mathrm{H}$ NMR $\left(400 \mathrm{MHz}, \mathrm{CDCl}_{3}\right)$ $\delta 7.77(\mathrm{~d}, J=8.4 \mathrm{~Hz}, 2 \mathrm{H}), 7.71(\mathrm{~d}, J=8.4 \mathrm{~Hz}, 2 \mathrm{H}), 3.07(\mathrm{t}, J=8.0 \mathrm{~Hz}, 2 \mathrm{H}), 1.73-$ $1.65(\mathrm{~m}, 2 \mathrm{H}), 1.37-1.23(\mathrm{~m}, 18 \mathrm{H}), 0.87(\mathrm{t}, J=6.4 \mathrm{~Hz}, 3 \mathrm{H}) ;{ }^{13} \mathrm{C} \mathrm{NMR}(100 \mathrm{MHz}$, $\left.\mathrm{CDCl}_{3}\right) \delta 138.2,132.5,129.5,128.8,56.2,31.8,29.5,29.4,29.3,29.2,29.1,28.9$, 28.1, 22.6, 22.5, 14.0; HRMS (ESI): Calcd for $\mathrm{C}_{18} \mathrm{H}_{30} \mathrm{BrO}_{2} \mathrm{~S}$ [M+H]: 389.1144, found: 389.1151 .<smiles>N#Cc1ccc(S(=O)(=O)CCCCC(=O)Nc2ccccc2)cc1</smiles>

\section{5-((4-Cyanophenyl)sulfonyl)- $N$-phenylpentanamide (4f)}

White solid (Reaction time: $12 \mathrm{~h}$, FCC eluent: EA/PE = 1:1, $96 \mathrm{mg}$, yield: 56\%), mp: $159-161{ }^{\circ} \mathrm{C}$; IR (KBr) v 1275, 1183, 1145, 850, $749 \mathrm{~cm}^{-1} ;{ }^{1} \mathrm{H}$ NMR (400 MHz, DMSO) $\delta 9.84(\mathrm{~s}, 1 \mathrm{H}), 8.13-8.07(\mathrm{~m}, 4 \mathrm{H}), 7.54(\mathrm{~d}, J=7.6 \mathrm{~Hz}, 2 \mathrm{H}), 7.29$ (t, $J=7.6$ $\mathrm{Hz}, 2 \mathrm{H}), 7.03$ (t, $J=7.2 \mathrm{~Hz}, 1 \mathrm{H}), 3.48(\mathrm{t}, J=7.6 \mathrm{~Hz}, 2 \mathrm{H}), 2.30$ (t, $J=6.8 \mathrm{~Hz}, 2 \mathrm{H})$, $1.67-1.56(\mathrm{~m}, 4 \mathrm{H}) ;{ }^{13} \mathrm{C}$ NMR (100 MHz, DMSO) $\delta 170.6,143.0,139.2,133.5,128.7$, 128.5, 123.1, 119.0, 117.6, 116.2, 53.8, 35.5, 23.6, 21.7; HRMS (ESI): Calcd for $\mathrm{C}_{18} \mathrm{H}_{19} \mathrm{~N}_{2} \mathrm{O}_{3} \mathrm{~S}[\mathrm{M}+\mathrm{H}]: 343.1111$, found: 343.1107 .<smiles></smiles>

\section{(Dodecylsulfonyl)cyclopropane (4j)}

White solid (Reaction time: $8 \mathrm{~h}$, FCC eluent: EA/PE = 1:3, $100 \mathrm{mg}$, yield: 73\%), mp: 47-49 ${ }^{\circ} \mathrm{C}$; IR (KBr) v 1244, 1130, 1151, $697 \mathrm{~cm}^{-1} ;{ }^{1} \mathrm{H}$ NMR $\left(400 \mathrm{MHz}, \mathrm{CDCl}_{3}\right) \delta$ 3.05-3.01 (m, 2H), 2.41-2.35 (m, 1H), 1.92-1.83 (m, 2H), 1.48-1.41 (m, 2H), 1.30$1.21(\mathrm{~m}, 18 \mathrm{H}), 1.06-1.01(\mathrm{~m}, 2 \mathrm{H}), 0.88(\mathrm{t}, J=6.4 \mathrm{~Hz}, 3 \mathrm{H}) ;{ }^{13} \mathrm{C}$ NMR $(100 \mathrm{MHz}$, $\left.\mathrm{CDCl}_{3}\right) \delta 53.9,31.8,29.5,29.4,29.2,29.1,29.0,28.4,22.6,22.1,14.0,4.4$; HRMS (ESI): Calcd for $\mathrm{C}_{15} \mathrm{H}_{30} \mathrm{NaO}_{2} \mathrm{~S}$ [M+Na]: 297.1860, found: 297.1859 .

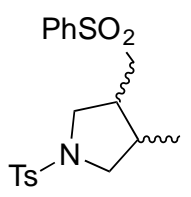

\section{Phenyl- $\lambda^{3}$-sulfanone compound} with

\section{3-(( $\lambda^{1}$-oxidanyl)methyl)-4-methyl-1-tosylpyrrolidine (7)}

White solid (Reaction time: $5 \mathrm{~h}$, FCC eluent: EA/PE $=1: 2,95 \mathrm{mg}$, yield: 48\%), mp: 141-143 ${ }^{\circ} \mathrm{C}$; IR (KBr) v 1305, 1244, 1161, 817, $747 \mathrm{~cm}^{-1} ;{ }^{1} \mathrm{H}$ NMR (400 MHz, $\left.\mathrm{CDCl}_{3}\right) \delta 7.86-7.84(\mathrm{~m}, 2 \mathrm{H}), 7.71-7.66(\mathrm{~m}, 3 \mathrm{H}), 7.57(\mathrm{t}, J=7.6 \mathrm{~Hz}, 2 \mathrm{H}), 7.34(\mathrm{~d}, J=$ $8.0 \mathrm{~Hz}, 2 \mathrm{H}), 3.66-3.49(\mathrm{~m}, 1 \mathrm{H}), 3.34-3.30(\mathrm{~m}, 1 \mathrm{H}), 3.19-3.15(\mathrm{~m}, 1 \mathrm{H}), 3.09-2.88(\mathrm{~m}$, 2H), 2.86-2.69 (m, 1H), 2.55-2.47 (m, 1H), 2.44 (s, 3H), 2.35-2.28 (m, 1H), 0.89- 
$0.74(\mathrm{~m}, 3 \mathrm{H}) ;{ }^{13} \mathrm{C} \mathrm{NMR}\left(100 \mathrm{MHz}, \mathrm{CDCl}_{3}\right) \delta 143.5,139.0,134.0,133.5,129.7,129.4$, 127.7, 127.4, 127.3, 58.2, 54.8, 54.0, 53.5, 52.5, 50.3, 39.8, 38.5, 35.9, 35.4, 21.4, 15.7, 13.2; HRMS (ESI): Calcd for $\mathrm{C}_{19} \mathrm{H}_{24} \mathrm{NO}_{4} \mathrm{~S}_{2}[\mathrm{M}+\mathrm{H}]$ : 394.1141, found: 394.1138.<smiles>O=S(=O)(C/C=C/CO)c1ccccc1</smiles>

(E)-4-(Phenylsulfonyl)but-2-en-1-ol (8) ${ }^{13}$

Colorless oil (Reaction time: 8 h, FCC eluent: EA/PE $=1: 1,55 \mathrm{mg}$, yield: $52 \%$ ), $\mathrm{R}_{\mathrm{f}}=$ $0.42(\mathrm{EA} / \mathrm{PE}=1: 1), \mathrm{IR}(\mathrm{KBr}) \vee 3420,1241,733,689 \mathrm{~cm}^{-1} ;{ }^{1} \mathrm{H}$ NMR $(400 \mathrm{MHz}$, $\left.\mathrm{CDCl}_{3}\right) \delta 7.87(\mathrm{~d}, J=7.6 \mathrm{~Hz}, 2 \mathrm{H}), 7.65(\mathrm{t}, J=7.6 \mathrm{~Hz}, 1 \mathrm{H}), 7.56(\mathrm{t}, J=7.6 \mathrm{~Hz}, 2 \mathrm{H})$, $6.02(\mathrm{~d}, J=5.5 \mathrm{~Hz}, 0.08 \mathrm{H}), 5.74-5.71(\mathrm{~m}, 1.83 \mathrm{H}), 5.46(\mathrm{~d}, J=9.2 \mathrm{~Hz}, 0.09 \mathrm{H}), 4.08$ $(\mathrm{d}, J=4.4 \mathrm{~Hz}, 1.80 \mathrm{H}), 4.02(\mathrm{~d}, J=5.2 \mathrm{~Hz}, 0.2 \mathrm{H}), 3.98(\mathrm{~d}, J=8.0 \mathrm{~Hz}, 0.19 \mathrm{H}), 3.81$ $(\mathrm{d}, J=6.8 \mathrm{~Hz}, 1.84 \mathrm{H}), 2.65(\mathrm{~s}, 1 \mathrm{H}) ;{ }^{13} \mathrm{C} \mathrm{NMR}\left(100 \mathrm{MHz}, \mathrm{CDCl}_{3}\right) \delta 140.0,138.5$, 138.2, 133.8, 129.2, 129.1, 128.2, 116.2, 62.2, 62.1, 59.5, 58.3, 54.9; HRMS (ESI): Calcd for $\mathrm{C}_{10} \mathrm{H}_{12} \mathrm{O}_{3} \mathrm{SNa}$ [M+Na]: 235.0399, found: 235.0405 .

$\mathrm{MeO} \mathrm{SO}_{2} \mathrm{Ph}-p-\mathrm{Cl}$

\section{Methyl (1S,2R)-2-((4-chlorophenyl)sulfonyl)cyclopentane-1-carboxylate (9)}

Colorless oil (Reaction time: 48 h, FCC eluent: EA/PE = 1:3, $51 \mathrm{mg}$, yield: $34 \%$ ), $\mathrm{R}_{\mathrm{f}}$ $=0.40(\mathrm{EA} / \mathrm{PE}=1: 3), \mathrm{IR}(\mathrm{KBr}) \vee 2875,1735,1229,830,756 \mathrm{~cm}^{-1} ;{ }^{1} \mathrm{H}$ NMR $(400$ $\left.\mathrm{MHz} \mathrm{CDCl}_{3}\right) \delta 7.84(\mathrm{~d}, J=8.0 \mathrm{~Hz}, 2 \mathrm{H}), 7.54(\mathrm{~d}, J=8.4 \mathrm{~Hz}, 2 \mathrm{H}), 4.01-3.96(\mathrm{~m}, 1 \mathrm{H})$, $3.54(\mathrm{~s}, 1 \mathrm{H}), 3.25-3.20(\mathrm{~m}, 1 \mathrm{H}), 2.24-2.16(\mathrm{~m}, 2 \mathrm{H}), 2.08-1.99(\mathrm{~m}, 1 \mathrm{H}), 1.83-1.76(\mathrm{~m}$, $3 \mathrm{H}) ;{ }^{13} \mathrm{C} \mathrm{NMR}\left(100 \mathrm{MHz}, \mathrm{CDCl}_{3}\right) \delta 173.7,140.4,136.7,130.0,129.4,66.2,52.2$, 45.1, 31.9, 27.2, 25.7; HRMS (ESI): Calcd for $\mathrm{C}_{13} \mathrm{H}_{16} \mathrm{ClO}_{4} \mathrm{~S}$ [M+H]: 303.0452, found: 303.0451 .

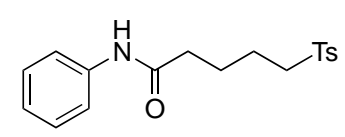

\section{$N$-Phenyl-5-tosylpentanamide (10)}

White solid (0.2 mmol scale, Reaction time: $4 \mathrm{~h}, \mathrm{FCC}$ eluent: $\mathrm{EA} / \mathrm{PE}=1: 1,65 \mathrm{mg}$, yield: 98\%), mp: $118-119^{\circ} \mathrm{C}$; IR (KBr) v 2092, 1641, $664 \mathrm{~cm}^{-1}$; ${ }^{1} \mathrm{H}$ NMR (400 MHz, $\left.\mathrm{CDCl}_{3}\right) \delta 7.81(\mathrm{~s}, 1 \mathrm{H}), 7.75(\mathrm{~d}, J=8.4 \mathrm{~Hz}, 2 \mathrm{H}), 7.49(\mathrm{~d}, J=8.0 \mathrm{~Hz}, 2 \mathrm{H}), 7.32(\mathrm{~d}, J=$ $8.0 \mathrm{~Hz}, 2 \mathrm{H}), 7.27(\mathrm{t}, J=8.0 \mathrm{~Hz}, 2 \mathrm{H}), 7.07(\mathrm{t}, J=7.6 \mathrm{~Hz}, 1 \mathrm{H}), 3.10(\mathrm{t}, J=7.2 \mathrm{~Hz}, 2 \mathrm{H})$, $2.42(\mathrm{~s}, 3 \mathrm{H}), 2.34(\mathrm{t}, J=6.8 \mathrm{~Hz}, 2 \mathrm{H}), 1.79-1.77(\mathrm{~m}, 4 \mathrm{H}) ;{ }^{13} \mathrm{C} \mathrm{NMR}\left(100 \mathrm{MHz}, \mathrm{CDCl}_{3}\right)$ $\delta 170.5,144.8,137.9,135.9,129.9,128.8,127.9,124.1,119.8,55.9,36.5,24.0,22.2$, 21.6; HRMS (ESI): Calcd for $\mathrm{C}_{18} \mathrm{H}_{22} \mathrm{NO}_{3} \mathrm{~S}[\mathrm{M}+\mathrm{H}]: 332.1315$, found: 332.1313 . 


\section{References}

1. Lowry, M. S.; Goldsmith, J. I.; Slinker, J. D.; Rohl, R.; Pascal, R. A.; Malliaras, G. G.; Bernhard, S. Chem. Mater. 2005, 17, 5712-5719.

2. (a) Miller, D. C.; Choi, G. J.; Orbe, H. S.; Knowles, R. R. J. Am. Chem. Soc. 2015, 137, 13492-13495. (b) Wang, J.-J.; Yu, W. Chem. Eur. J. 2019, 25, 3510-3514.

3. Fustero, S.; Sánchez-Roselló, M.; Jiménez, D.; Sanz-Cervera, J. F.; Pozo, C.; Aceña, J. L. J. Org. Chem. 2006, 71, 2706-2714.

4. Meyer, A. U.; Jäger, S.; Hari, D.; P.; König, B. Adv. Synth. Catal. 2015, 357, 2050-2054.

5. Schleicher, K. D.; Jamison, T. F. Org. Lett. 2007, 9, 875-878.

6. Cismesia, M. A.; Yoon, T. P. Chem. Sci. 2015, 6, 5426-5434.

7. Ham,W. S.; Hillenbrand, J.; Jacq, J.; Genicot, C.; Ritter, T. Angew. Chem. Int. Ed. 2019, $58,532-536$.

8. Luis-Barrera, J.; LainaMartin, V.; Rigotti, T.; Peccati, F.; Solans-Monfort, X.; Sodupe, M.; Mas-Balleste, R.; Liras, M.; Aleman, J. Angew. Chem., Int. Ed. 2017, $56,7826-7830$.

9. Klauck, F. J. R.; James, M. J.; Glorius, F. Angew. Chem. Int. Ed. 2017, 56, 12336-12339.

10. (a) Kuhn, H. J.; Braslavsky, S. E.; Schmidt, R. Pure Appl. Chem. 2004, 76, 2105-2146. (b) Monalti, M.; Credi, A.; Prodi, L.; Gandolfi, M. T. Chemical Actinometry. Handbook of Photochemistry, 3rd Ed. 2006, 601-604.

11. Pohmakotr, M.; Ieawsuwan, W.; Tuchinda, P.; Kongsaeree, P.; Prabpai, S.; Reutrakul, V. Org. Lett. 2004, 4547-4550.

12. Heß, T. Raabe, G.; Gais, H.-J. Eur. J. Org. Chem. 2014, 7134-7147.

13. Sheldrake, H. M.; Wallace, T. W. Tetrahedron Lett. 2007, 48, 4407-4411. 


\section{${ }^{1} \mathrm{H}$ and ${ }^{13} \mathrm{C}$ NMR spectra}

\section{$N$-Ethyl- $N$-phenylpent-4-enamide (1j)}

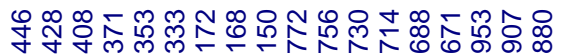

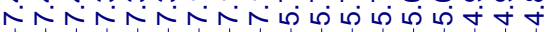

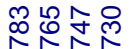
गं

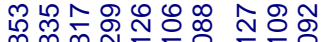<smiles>C=CCCC(=O)N(CC)c1ccccc1</smiles>

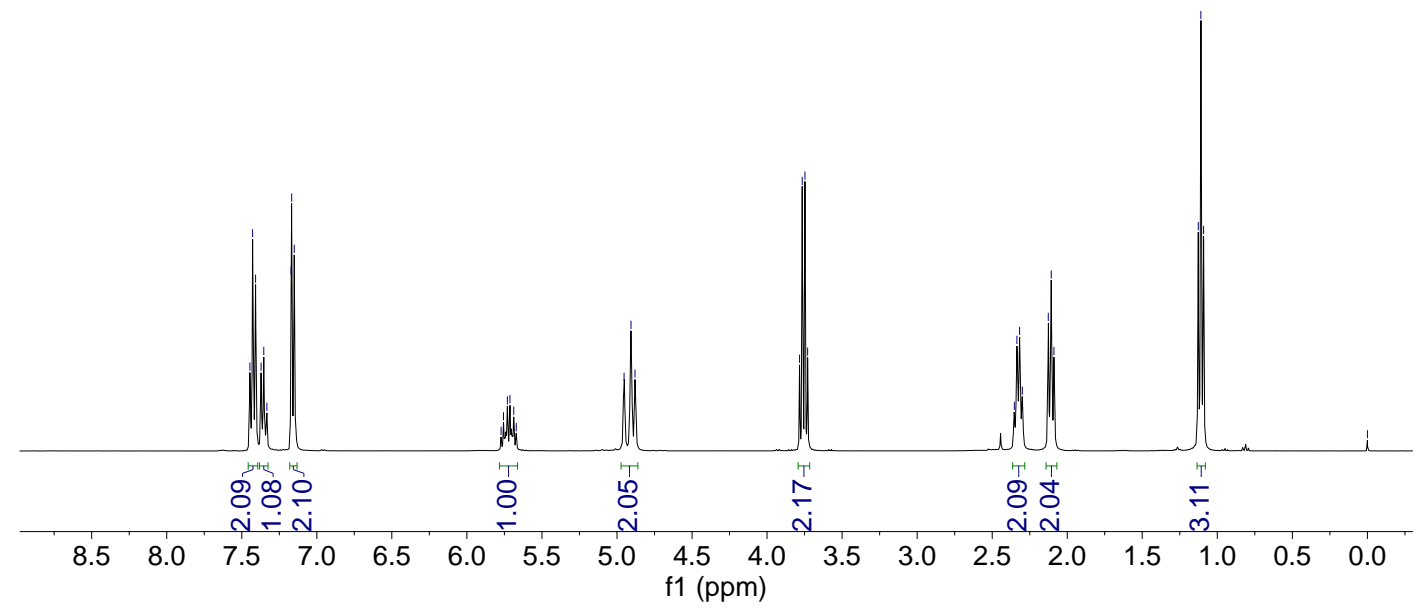

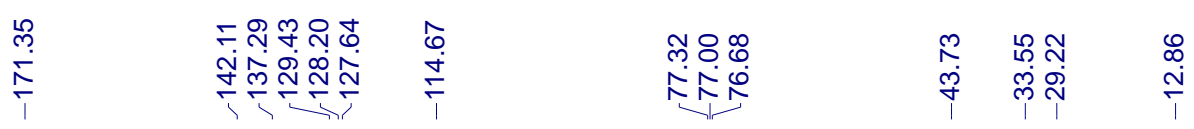

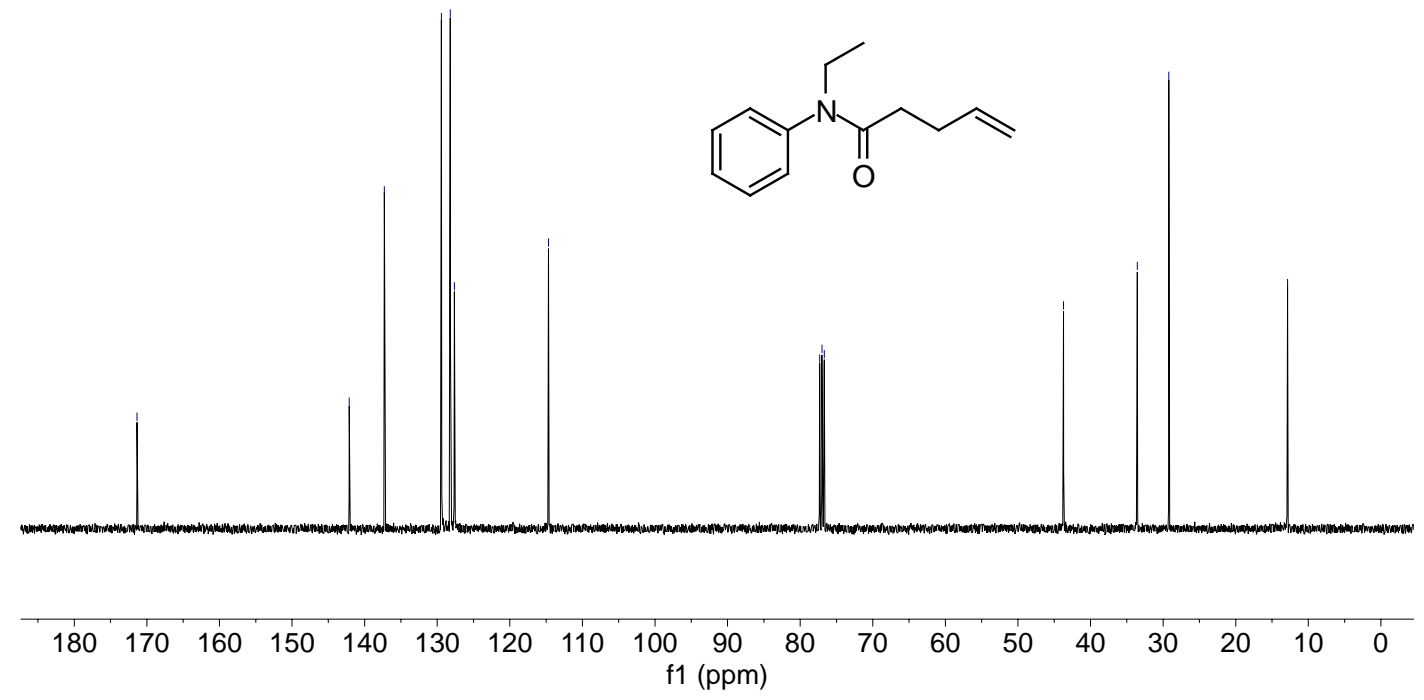




\section{$N$-Benzyl- $N$-phenylpent-4-enamide (1k)}

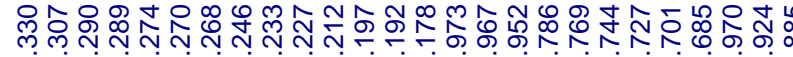

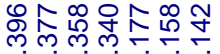

ヘึ N

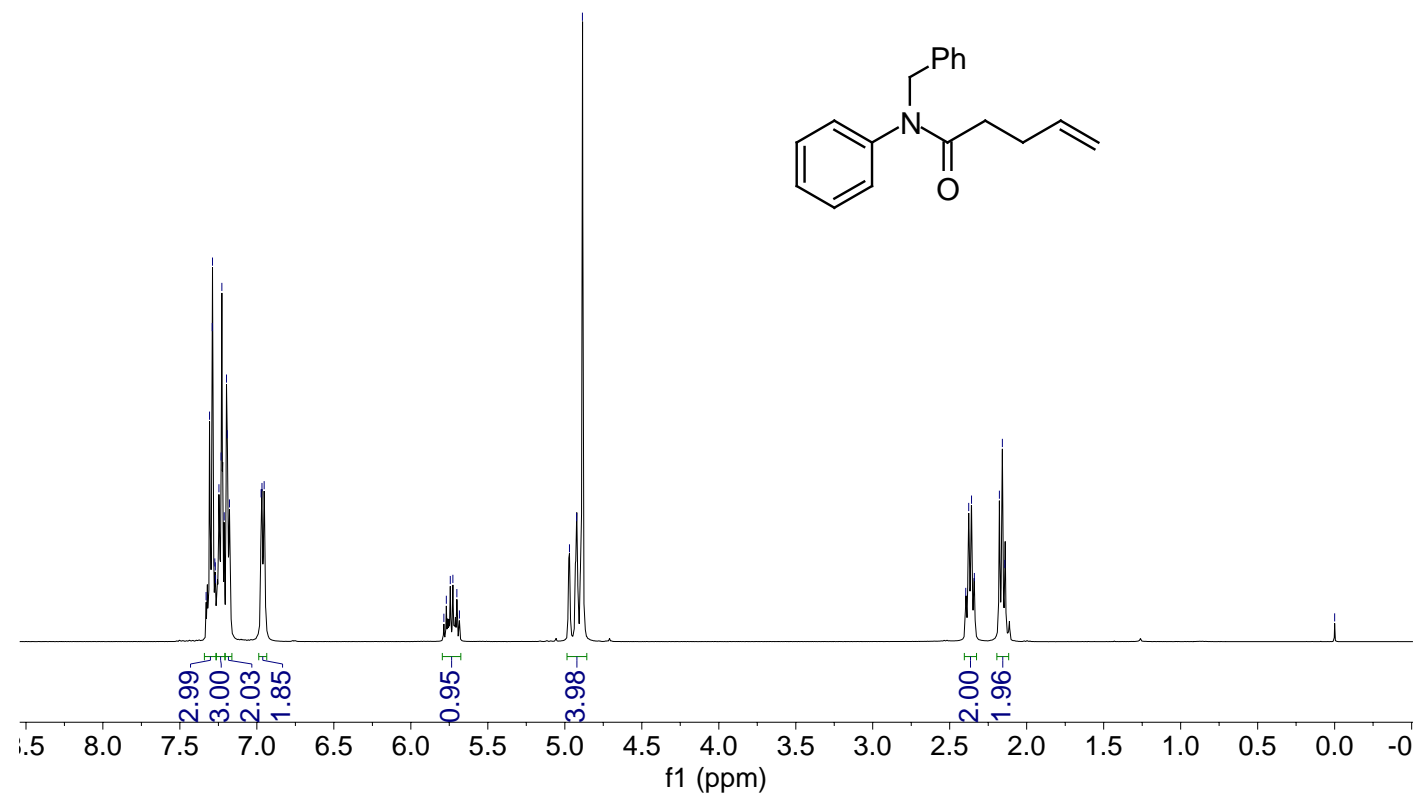

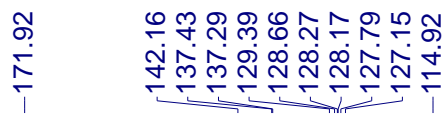

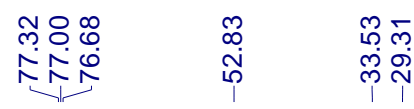

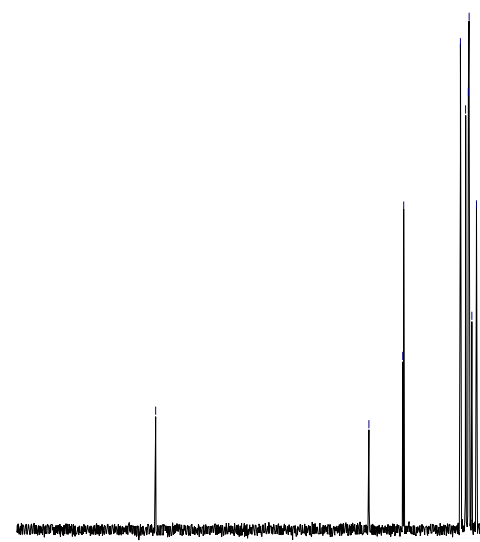<smiles>C=CCCC(=O)N(Cc1ccccc1)c1ccccc1</smiles>

$\begin{array}{lllllllllll}90 & 180 & 170 & 160 & 150 & 140 & 130 & 120 & 110 & 100 & 90\end{array}$

$\begin{array}{lllllllll}80 & 70 & 60 & 50 & 40 & 30 & 20 & 10 & 0\end{array}$

f1 (ppm) 


\section{$N$-Allyl- $N$-phenylpent-4-enamide (1I)}

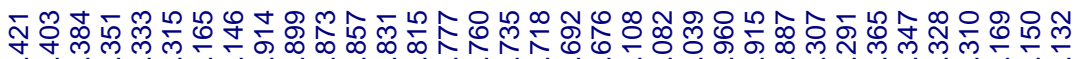

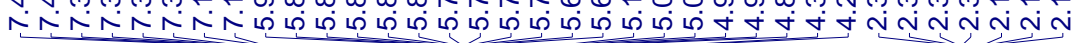<smiles>C=CCCC(=O)N(CC=C)c1ccccc1</smiles>

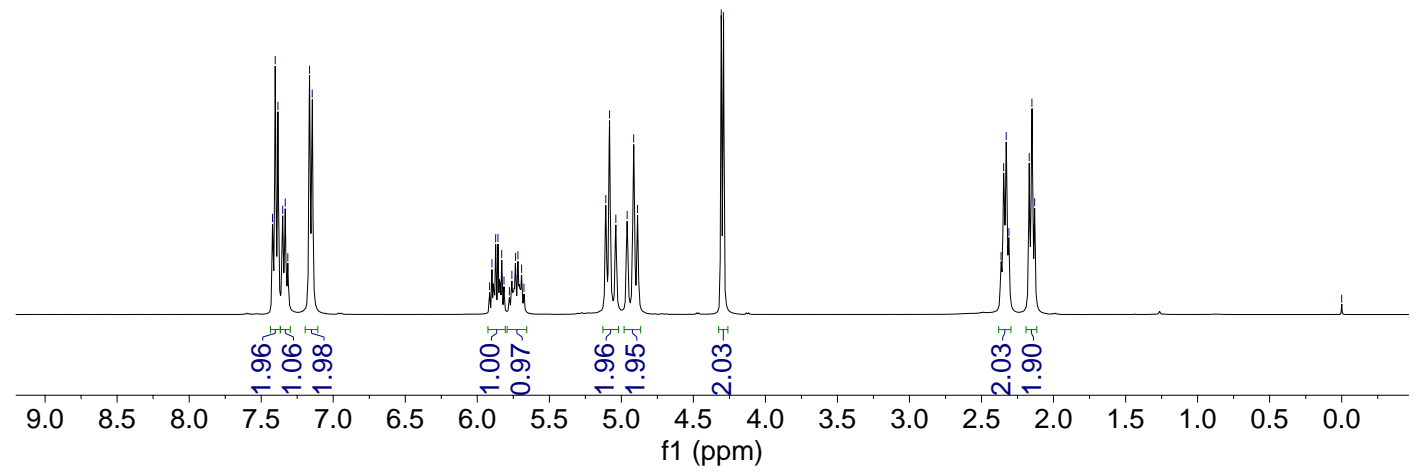

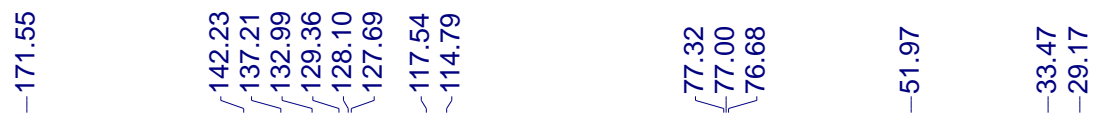<smiles>C=CCCC(=O)N(CC=C)c1ccccc1</smiles>

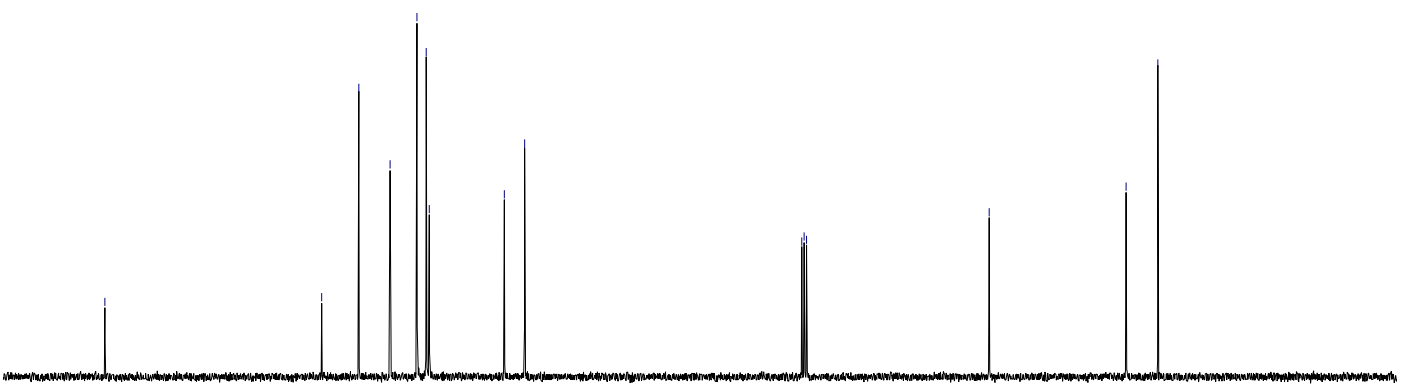

$\begin{array}{lllllllllllllllllll}180 & 170 & 160 & 150 & 140 & 130 & 120 & 110 & 100 \begin{array}{c}90 \\ \mathrm{f} 1(\mathrm{ppm})\end{array} & 80 & 70 & 60 & 50 & 40 & 30 & 20 & 10 & 0\end{array}$ 
But-3-en-1-yl 1-methyl-1H-pyrrole-2-carboxylate (1v)

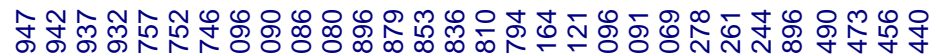

טف

$\stackrel{8}{8}$

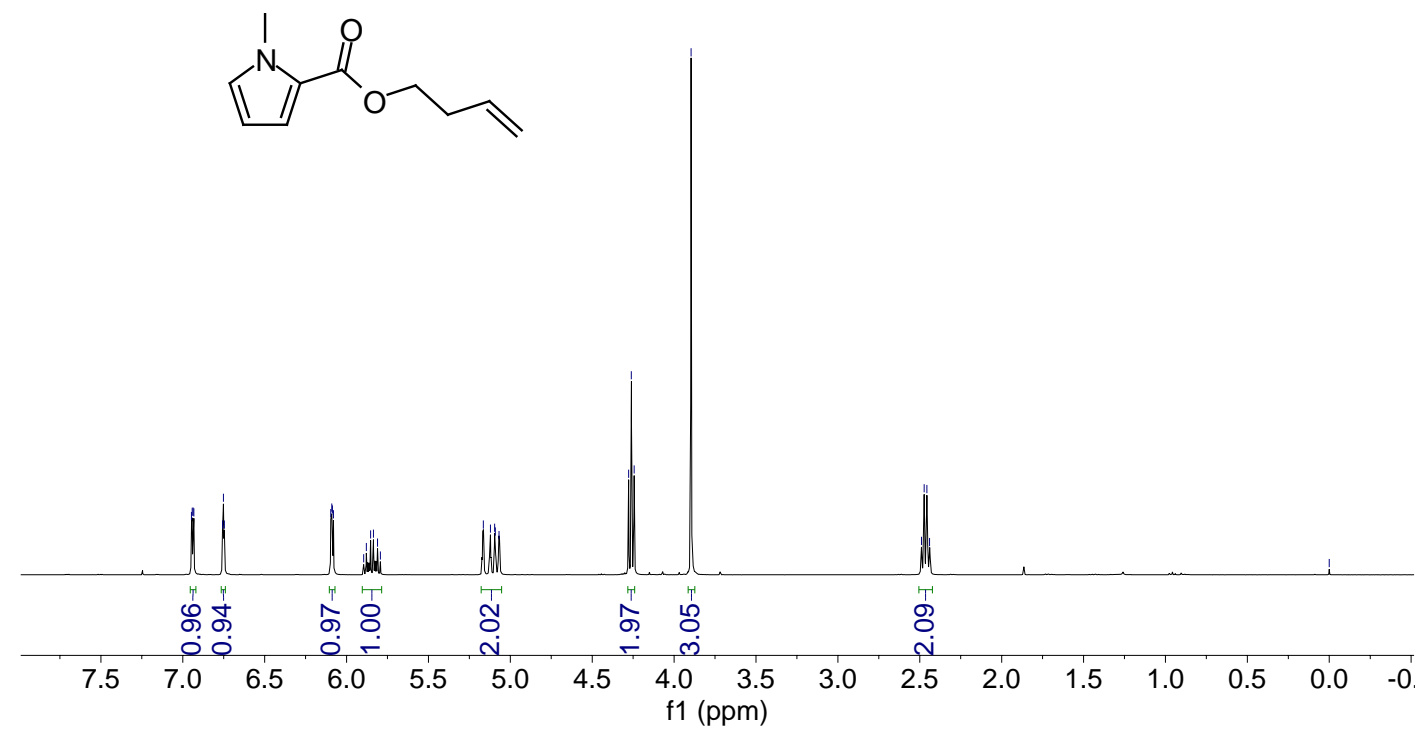

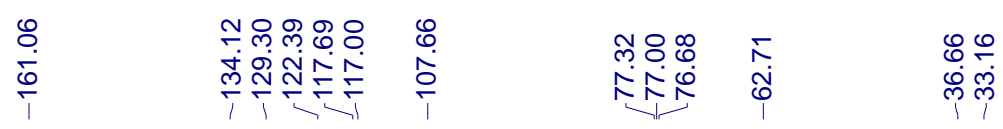<smiles>C=CCCOC(=O)c1cccn1C</smiles>

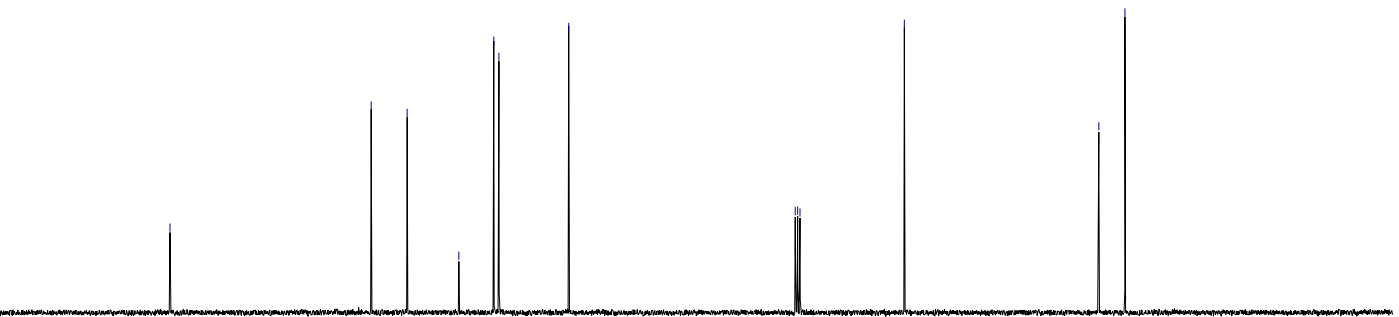

$\begin{array}{lllllllllllllllllll}180 & 170 & 160 & 150 & 140 & 130 & 120 & 110 & 100 & \begin{array}{c}90 \\ \mathrm{f} 1(\mathrm{ppm})\end{array} & 80 & 70 & 60 & 50 & 40 & 30 & 20 & 10 & 0\end{array}$ 

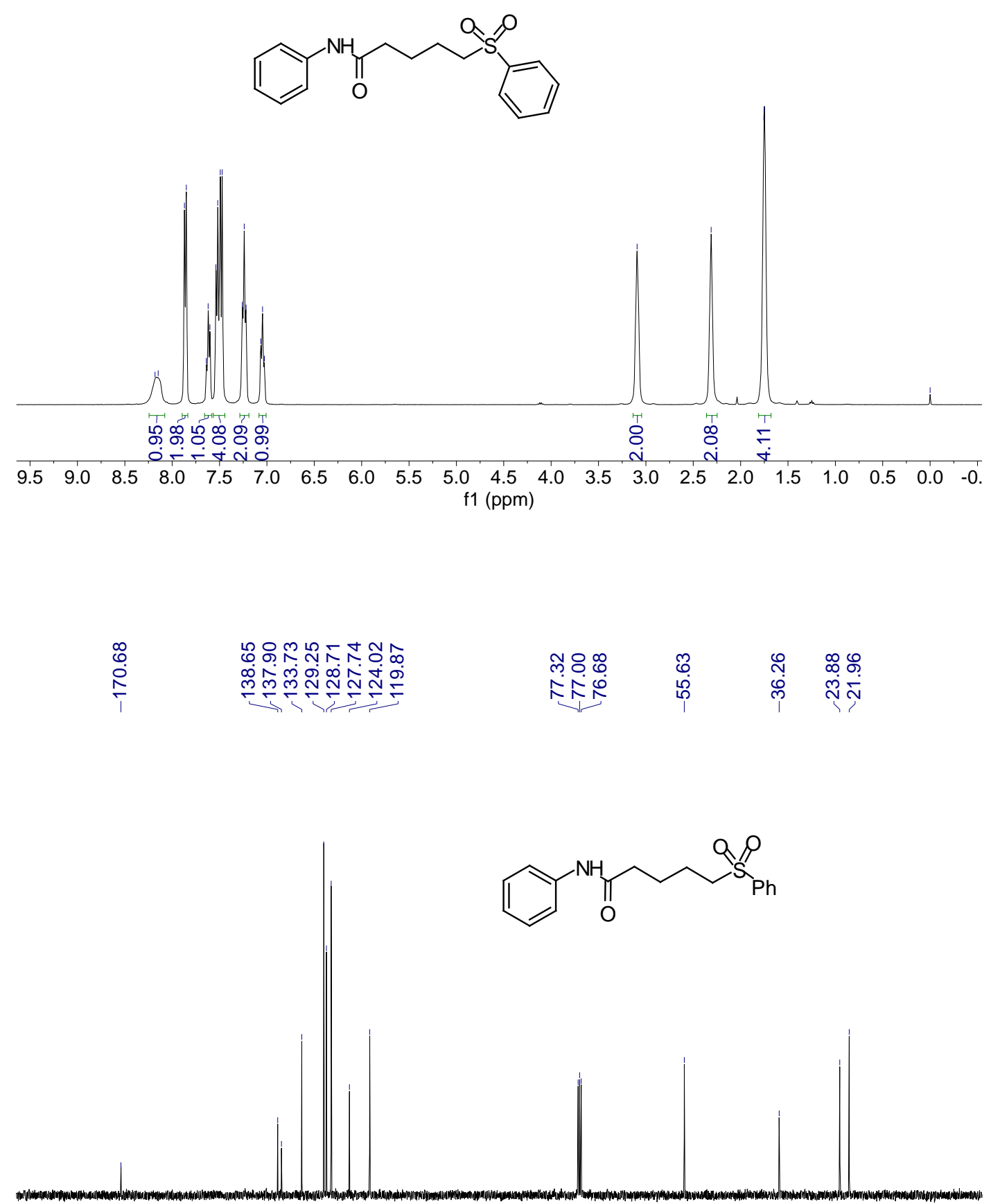

\begin{tabular}{|lllllllllllllllllll}
190 & 180 & 170 & 160 & 150 & 140 & 130 & 120 & 110 & $\begin{array}{c}100 \\
\mathrm{f} 1(\mathrm{ppm})\end{array}$ & $\begin{array}{c}90 \\
(\mathrm{ppm}\end{array}$ & 70 & 60 & 50 & 40 & 30 & 20 & 10 & 0
\end{tabular} 


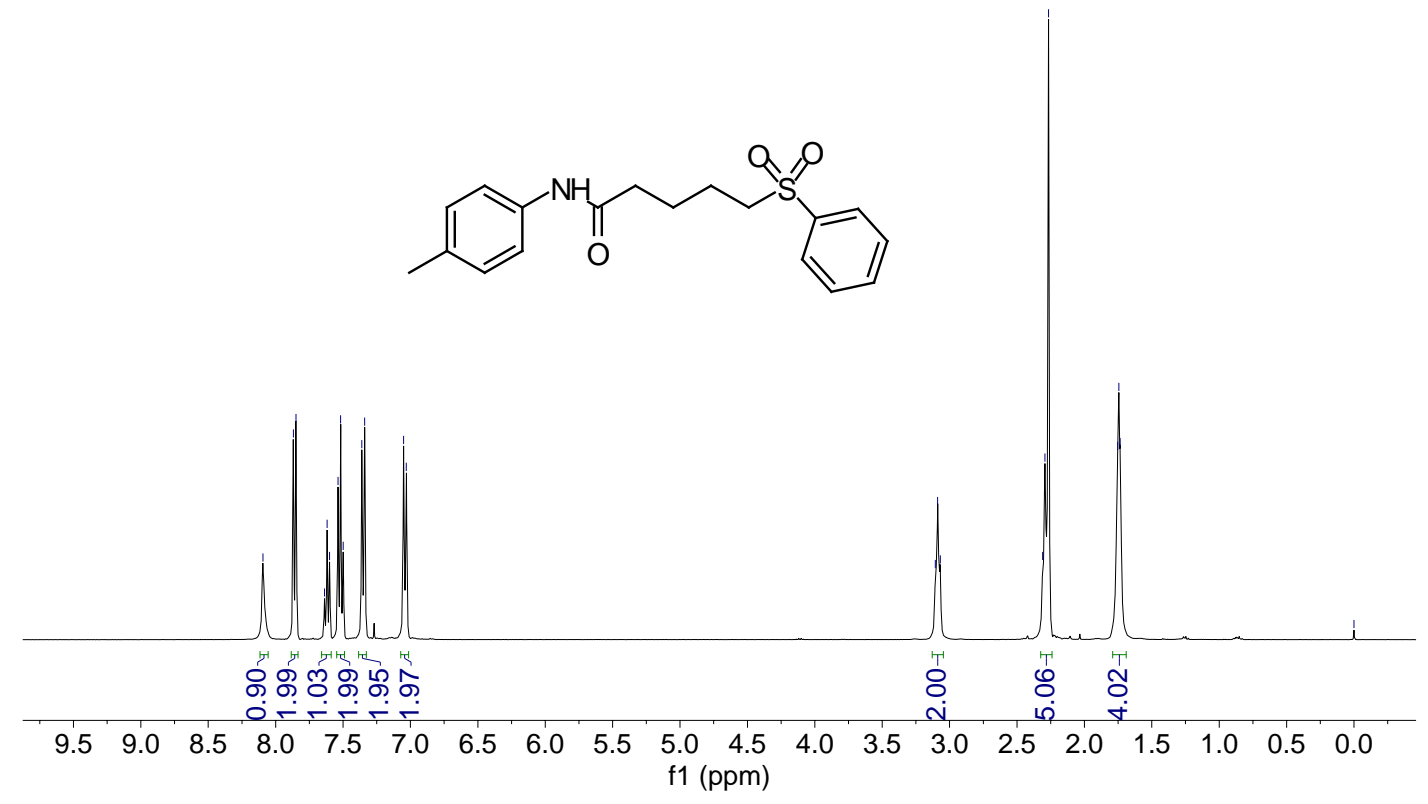

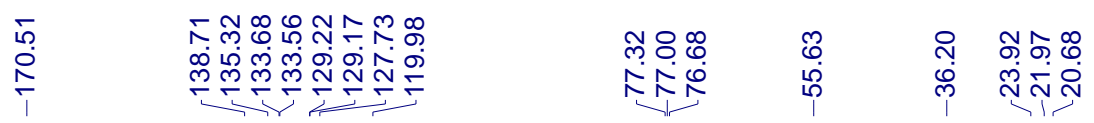

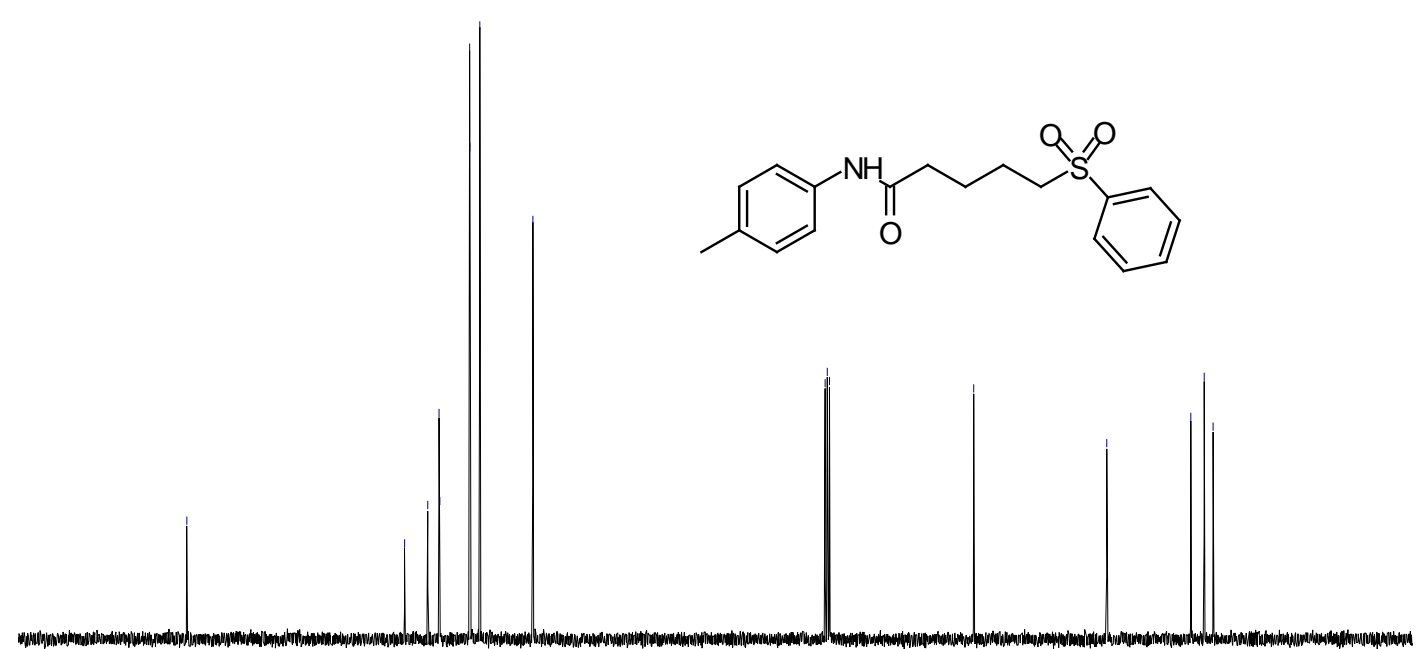


$N$-(4-Methoxyphenyl)-5-(phenylsulfonyl)pentanamide (3c)

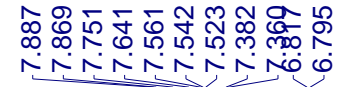

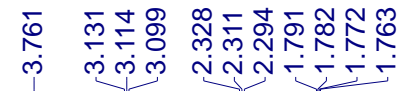

$\stackrel{8}{8}$

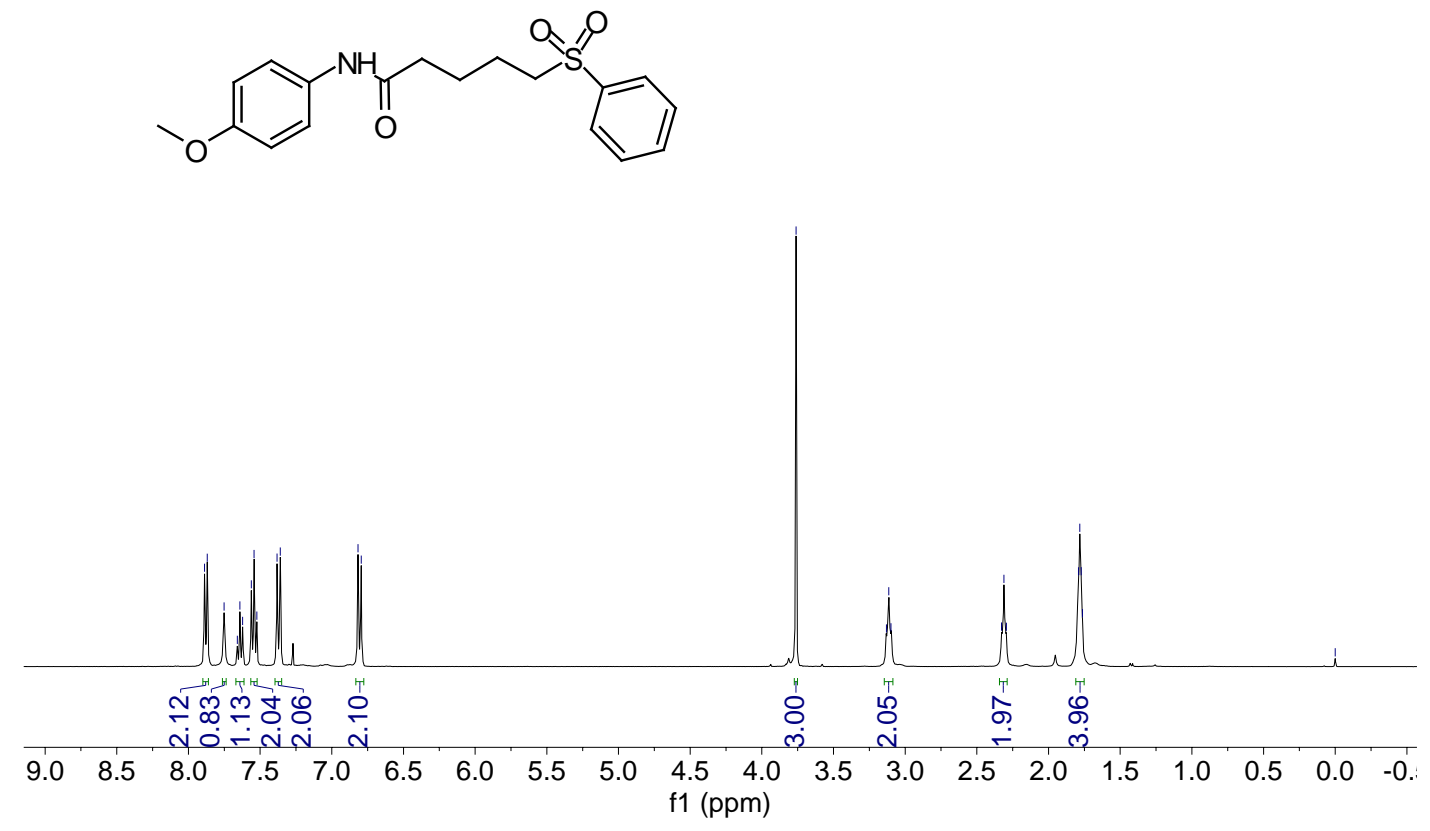

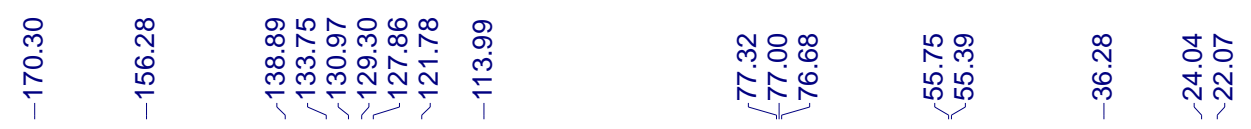

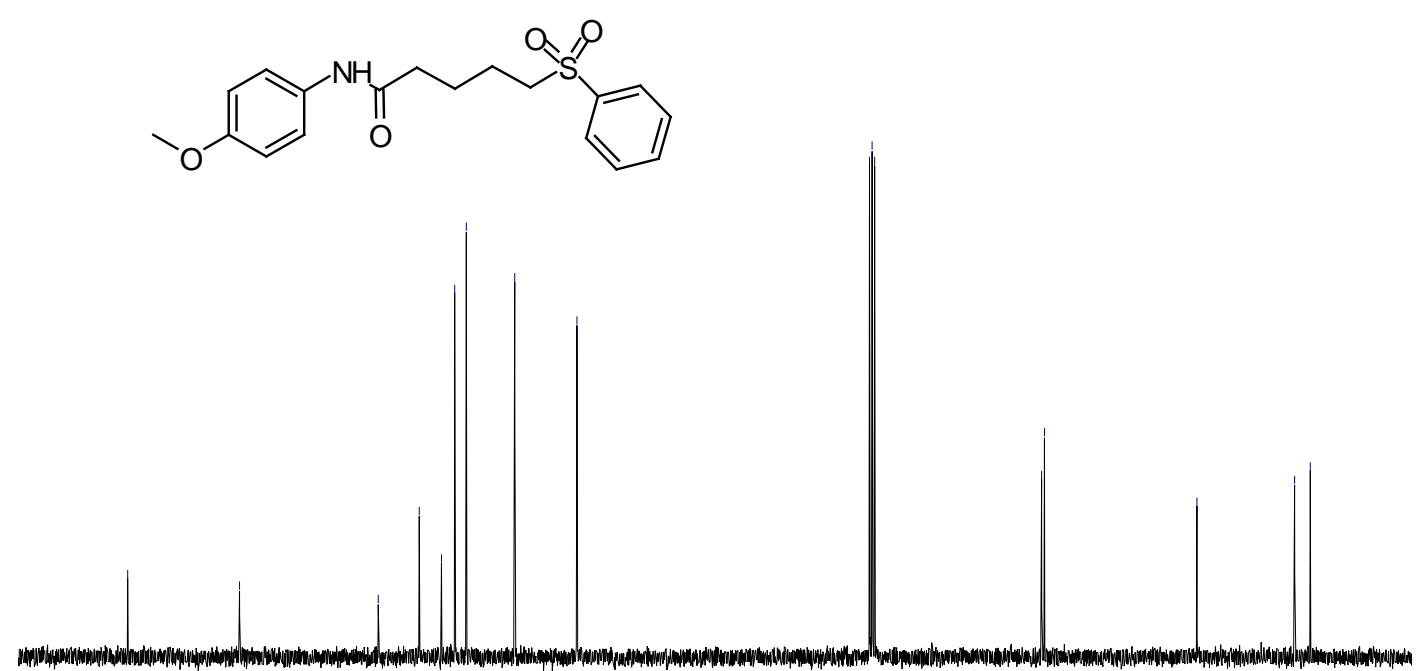

$\begin{array}{llllllllllllllllll}180 & 170 & 160 & 150 & 140 & 130 & 120 & 110 & \begin{array}{c}100 \\ \mathrm{f} 1(\mathrm{ppm})\end{array} & 80 & 70 & 60 & 50 & 40 & 30 & 20 & 11\end{array}$ 


\section{$N$-(4-Fluorophenyl)-5-(phenylsulfonyl)pentanamide (3d)}

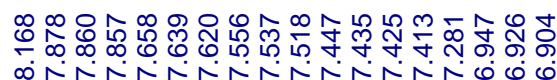

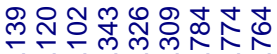

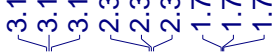

ஜ<smiles>O=C(CCCCS(=O)(=O)c1ccccc1)Nc1ccc(F)cc1</smiles>

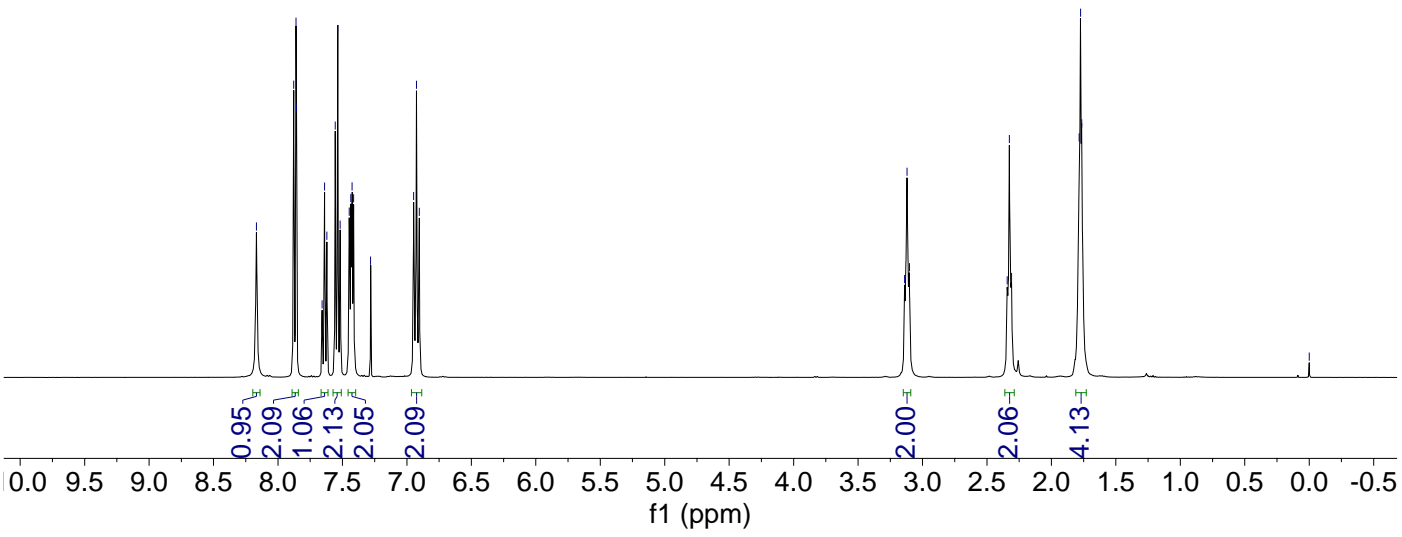

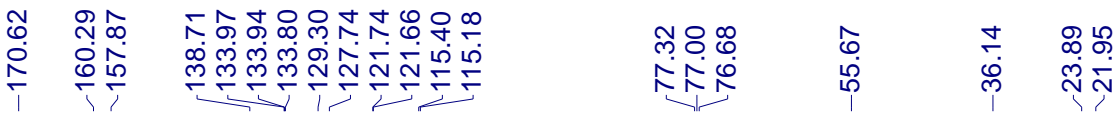<smiles>O=C(CCCCS(=O)(=O)c1ccccc1)Nc1ccc(F)cc1</smiles>

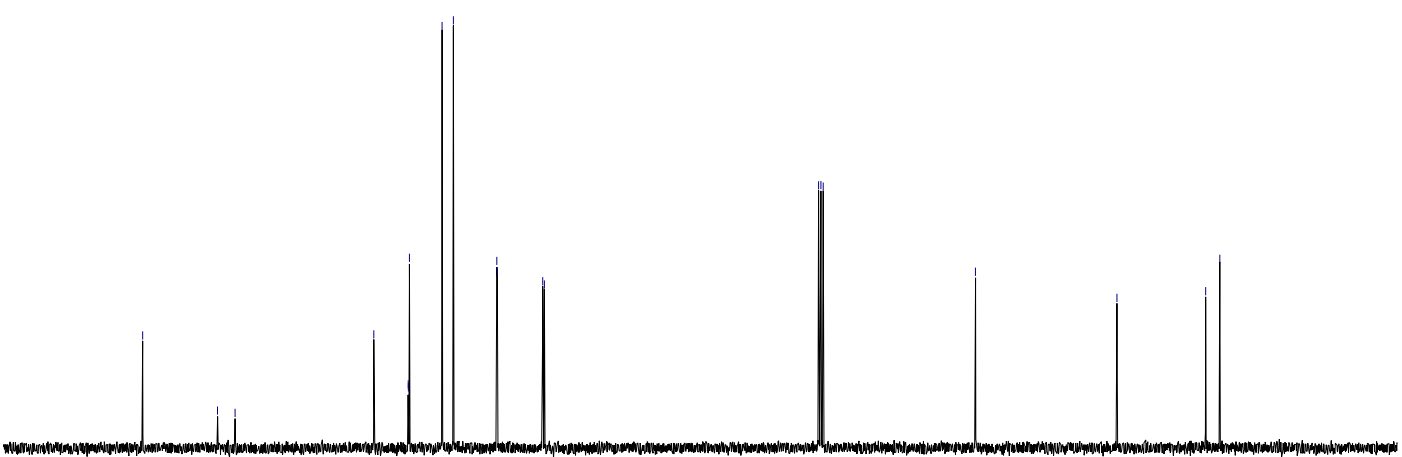

$\begin{array}{llllllllllllllllllllllll}180 & 170 & 160 & 150 & 140 & 130 & 120 & 110 & 100 & 90 & 80 & 70 & 60 & 50 & 40 & 30 & 20 & 10 & 0\end{array}$ 


\section{$N$-(4-Bromophenyl)-5-(phenylsulfonyl)pentanamide (3e)}

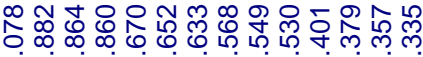

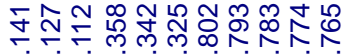

ம்

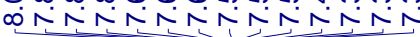<smiles>O=C(CCCCS(=O)(=O)c1ccccc1)Nc1ccc(Br)cc1</smiles>

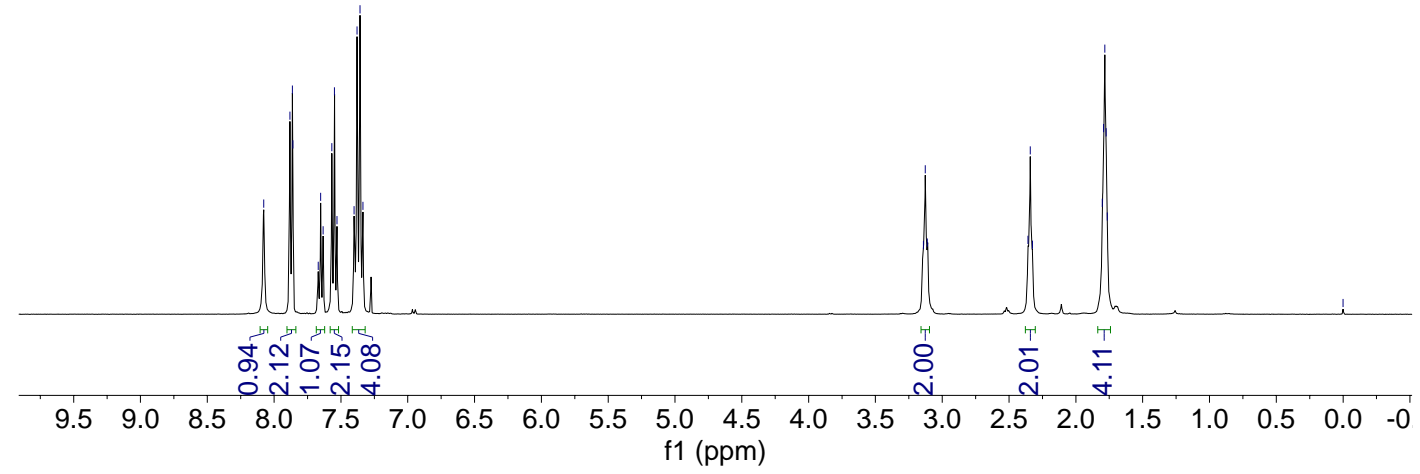

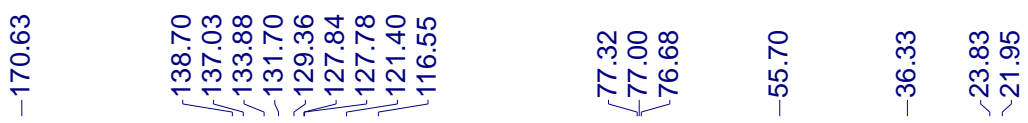

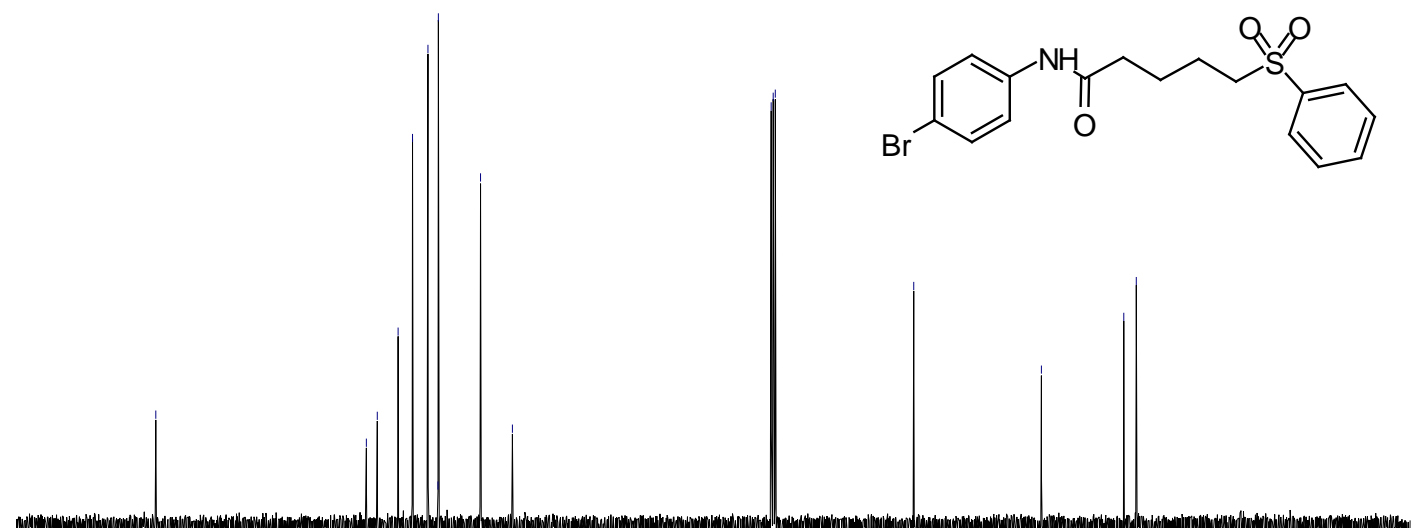

$\begin{array}{llllllllllllllllllllll}90 & 180 & 170 & 160 & 150 & 140 & 130 & 120 & 110 & 100 & 90 & 80 & 70 & 60 & 50 & 40 & 30 & 20 & 10 & 0 & -10\end{array}$ f1 (ppm) 
2-Methyl-5-(phenylsulfonyl)- $N$-(o-tolyl)pentanamide (3g)

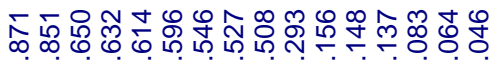

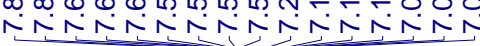

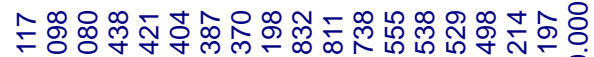

लं<smiles>Cc1ccccc1NC(=O)C(C)CCCS(=O)(=O)c1ccccc1</smiles>

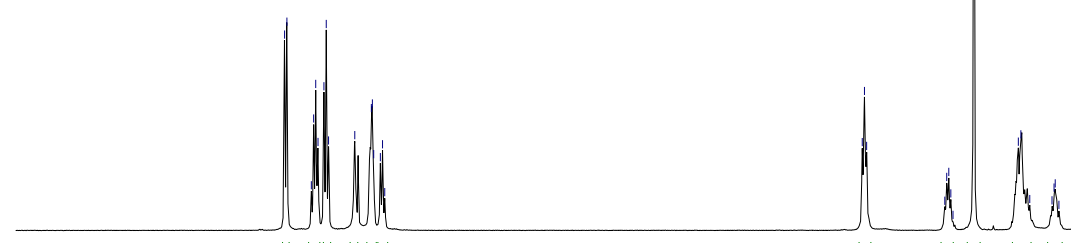

कृष्ठक

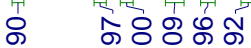

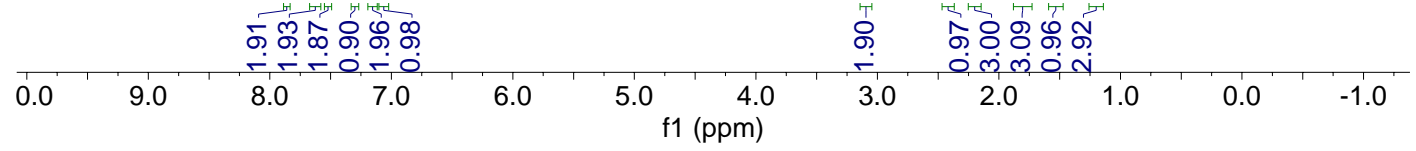

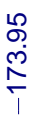<smiles>Cc1ccccc1NC(=O)C(C)CCCS(=O)(=O)c1ccccc1</smiles>

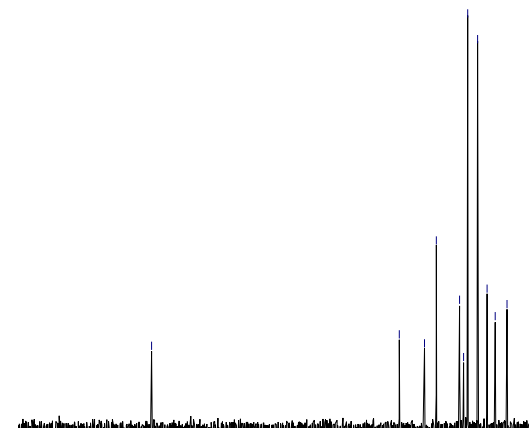

$\begin{array}{lllllllllll}190 & 180 & 170 & 160 & 150 & 140 & 130 & 120 & 110 & 100 & 90\end{array}$

$\begin{array}{lll}80 & 70 \quad 60\end{array}$

$\begin{array}{lll}50 & 40\end{array}$ $20 \quad 10 \quad 0$ 
2,2-Dimethyl-5-(phenylsulfonyl)- $N$-(o-tolyl)pentanamide (3h)

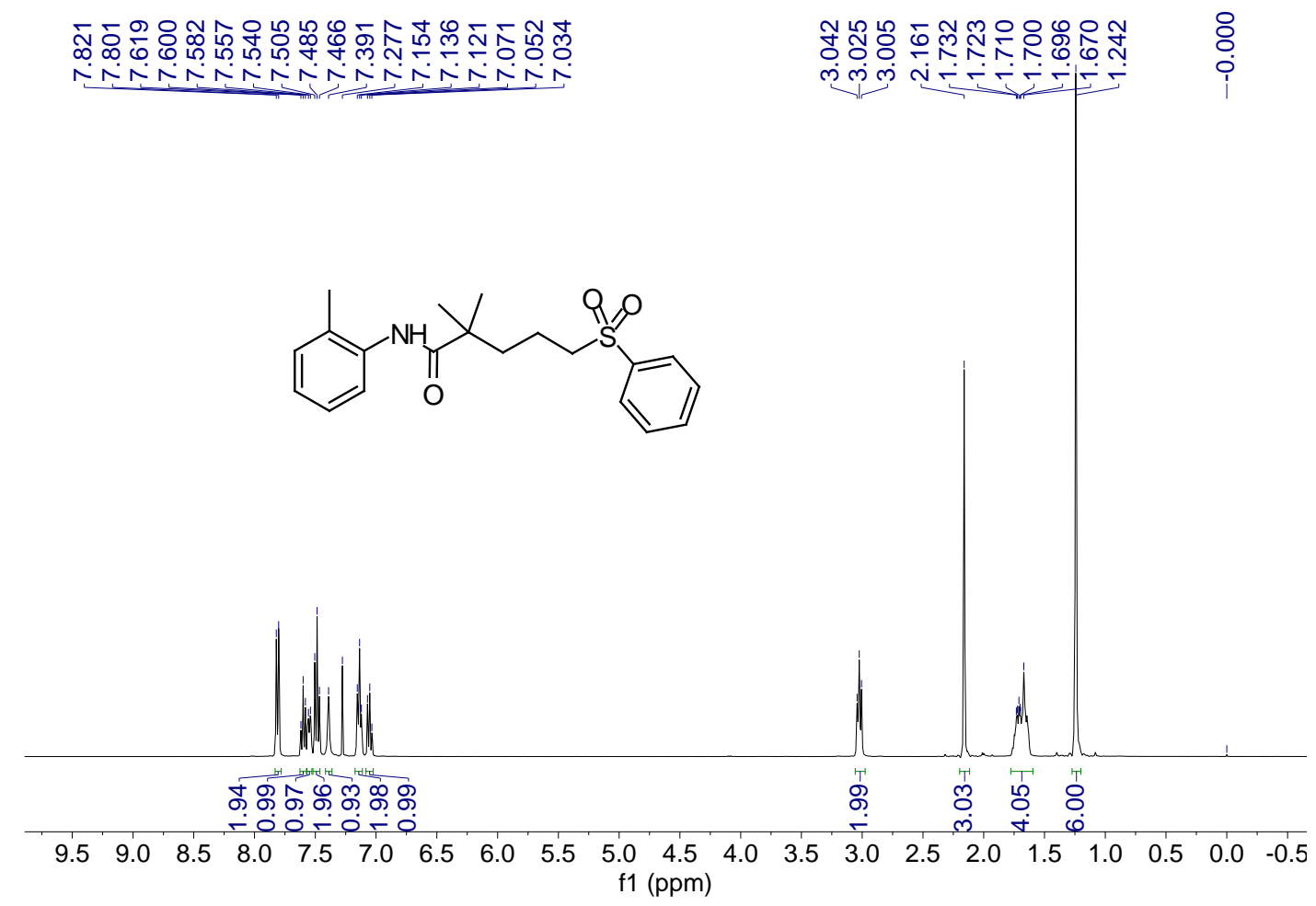

\begin{tabular}{|c|c|c|c|}
\hline 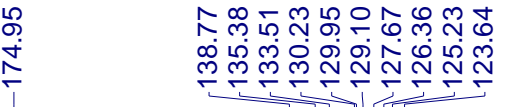 & 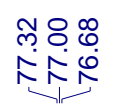 & $\begin{array}{l}\text { Oొ } \\
\text { م. }\end{array}$ & 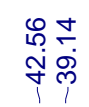 \\
\hline
\end{tabular}

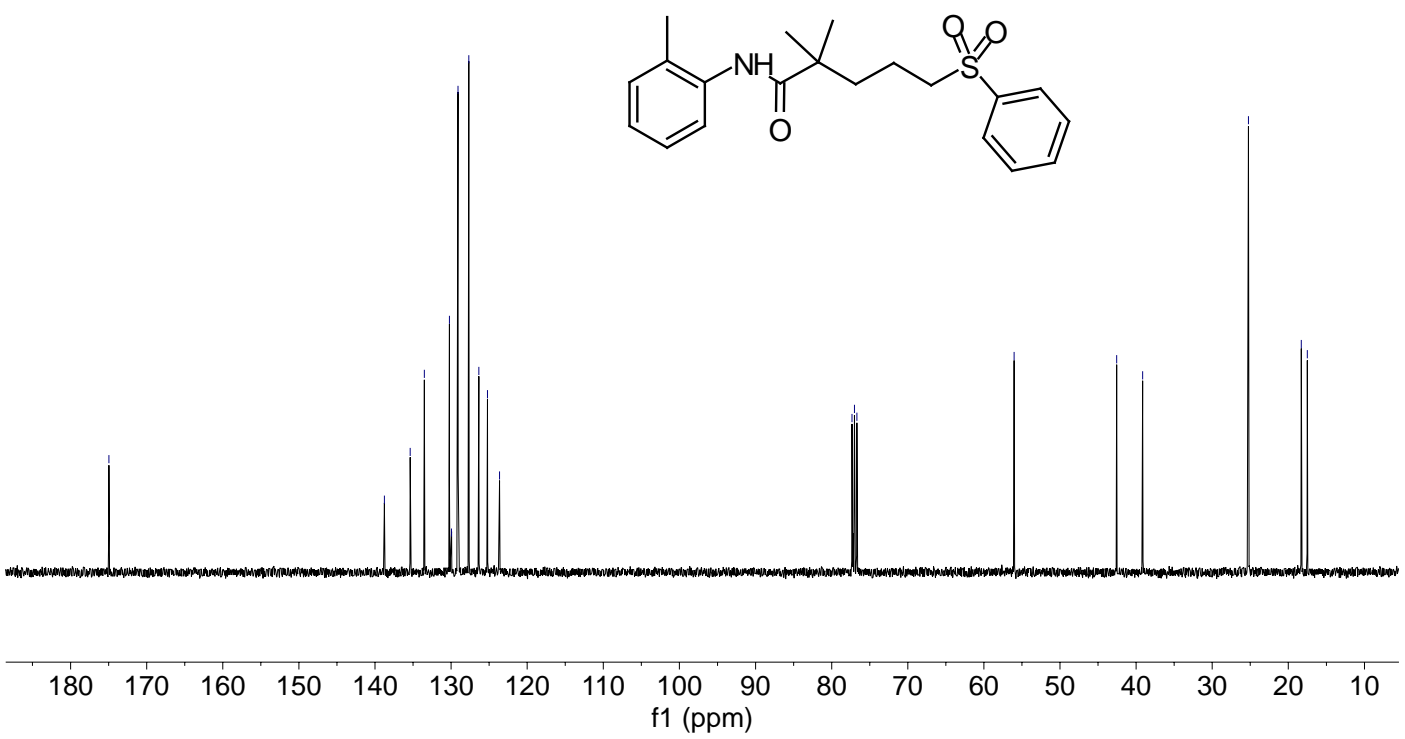


3,3-Dimethyl-5-(phenylsulfonyl)- $N$-(o-tolyl)pentanamide (3i)

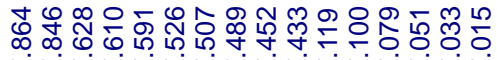

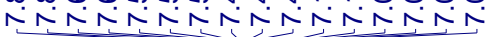

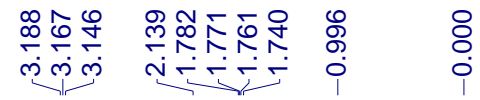<smiles>Cc1ccccc1NC(=O)CC(C)(C)CCS(=O)(=O)c1ccccc1</smiles>
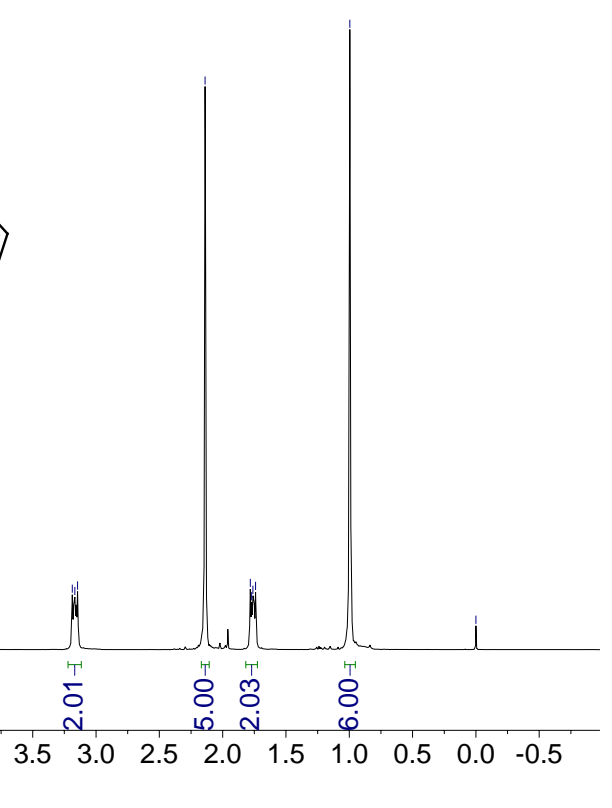

\begin{tabular}{|c|c|c|}
\hline 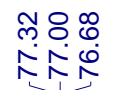 & $\begin{array}{l}\text { の } \\
\text { तิ } \\
\text { ஸे }\end{array}$ & 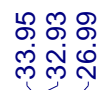 \\
\hline
\end{tabular}

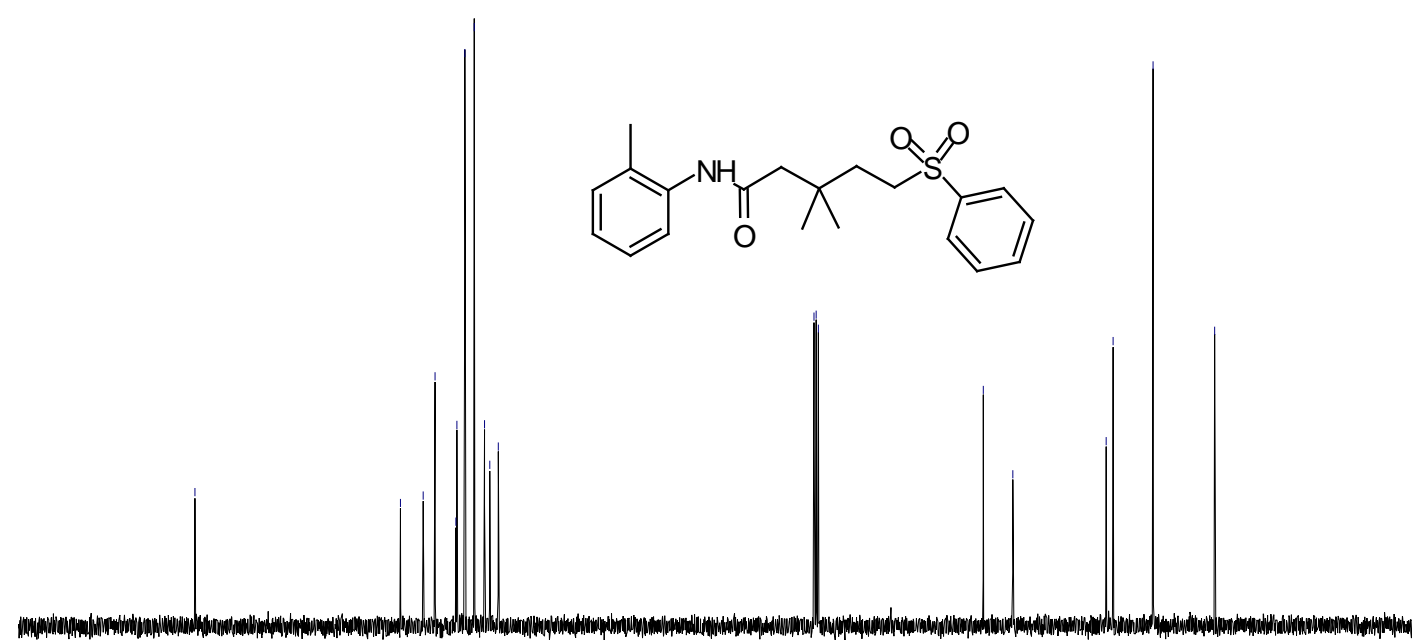

$\begin{array}{lllllllllllllllllllll}190 & 180 & 170 & 160 & 150 & 140 & 130 & 120 & 110 & 100 & 90 & 80 & 70 & 60 & 50 & 40 & 30 & 20 & 10 & 0 & -11\end{array}$ 


\section{$N$-Ethyl- $N$-phenyl-5-(phenylsulfonyl)pentanamide (3j)}

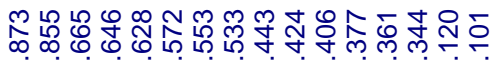

NiñNoñ

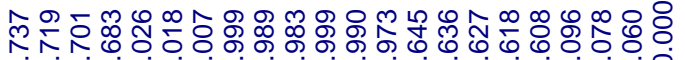
लें ले लें लं लं

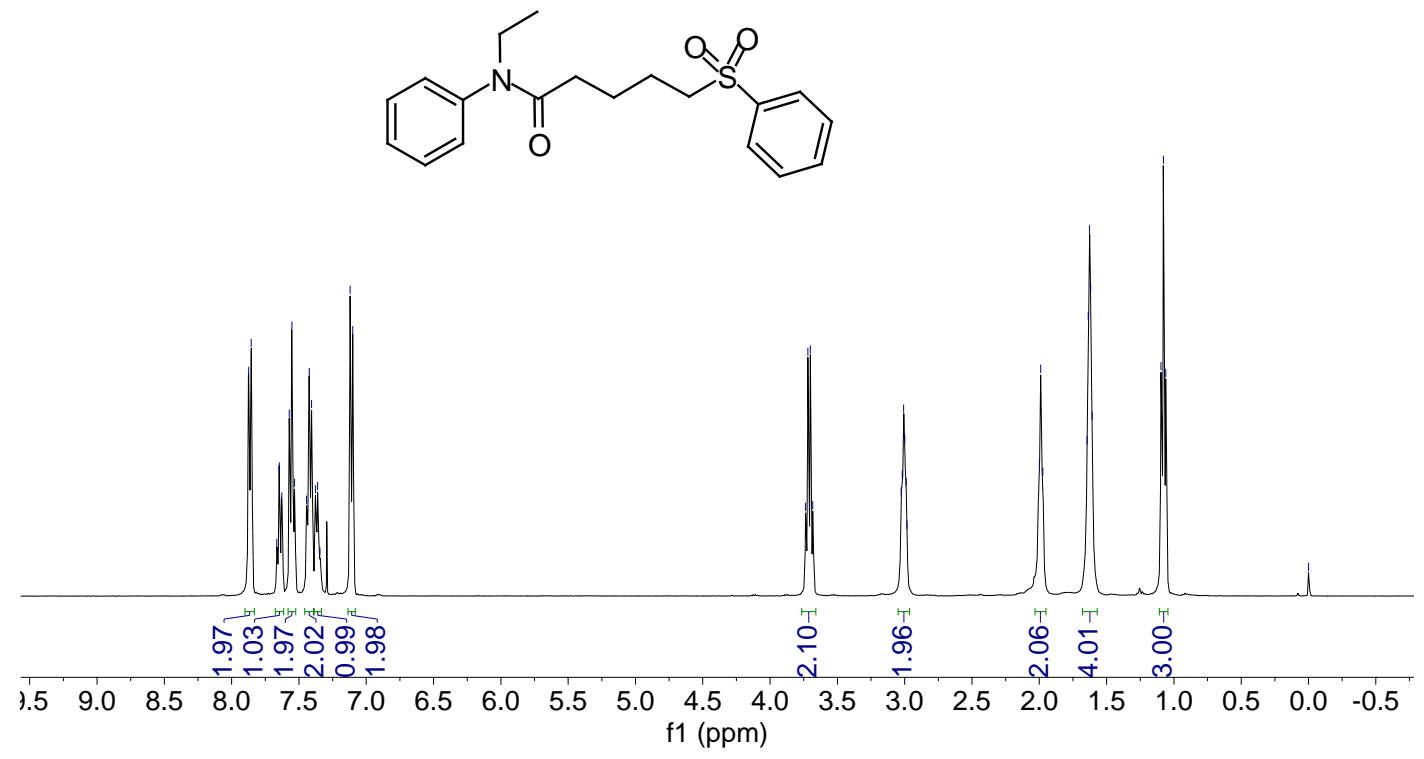

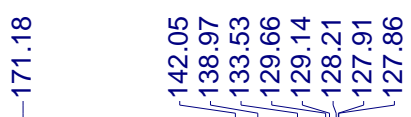

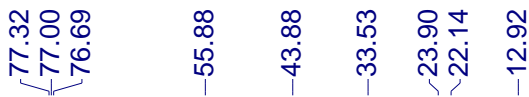

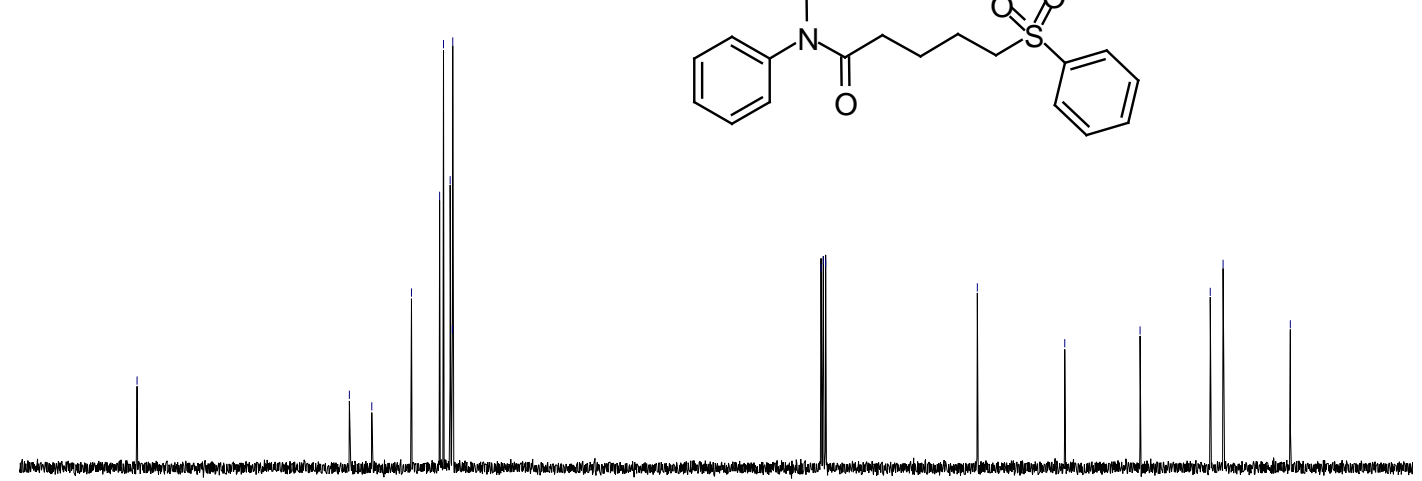

$\begin{array}{llllllllllllllllllll}180 & 170 & 160 & 150 & 140 & 130 & 120 & 110 & 100 & \begin{array}{c}90 \\ \mathrm{f} 1(\mathrm{ppm})\end{array} & 80 & 70 & 60 & 50 & 40 & 30 & 20 & 10 & 0\end{array}$ 


\section{$N$-Benzyl- $N$-phenyl-5-(phenylsulfonyl)pentanamide (3k)}

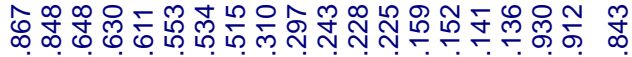

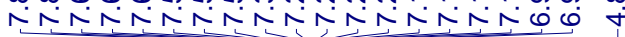
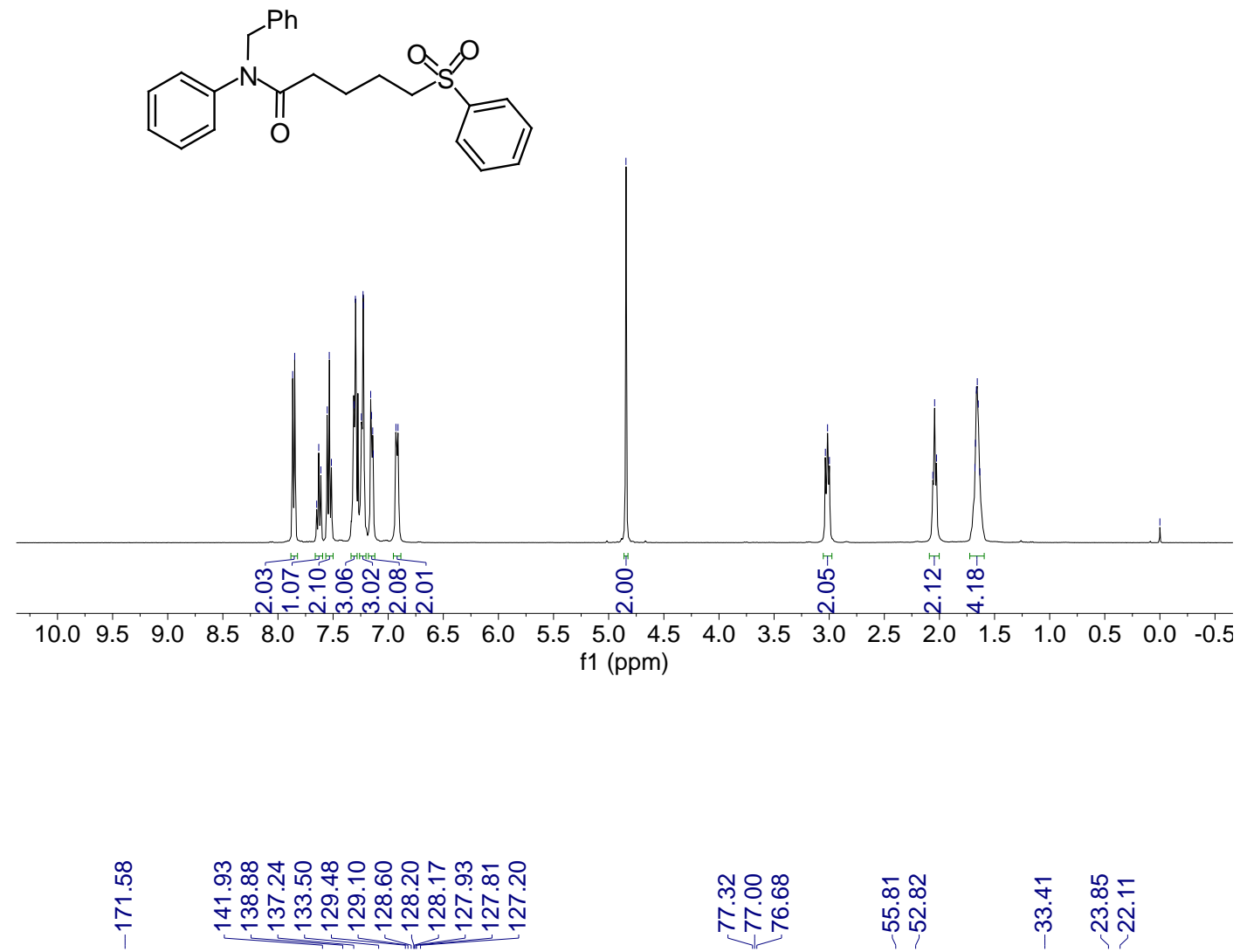

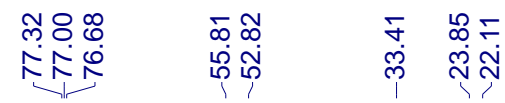

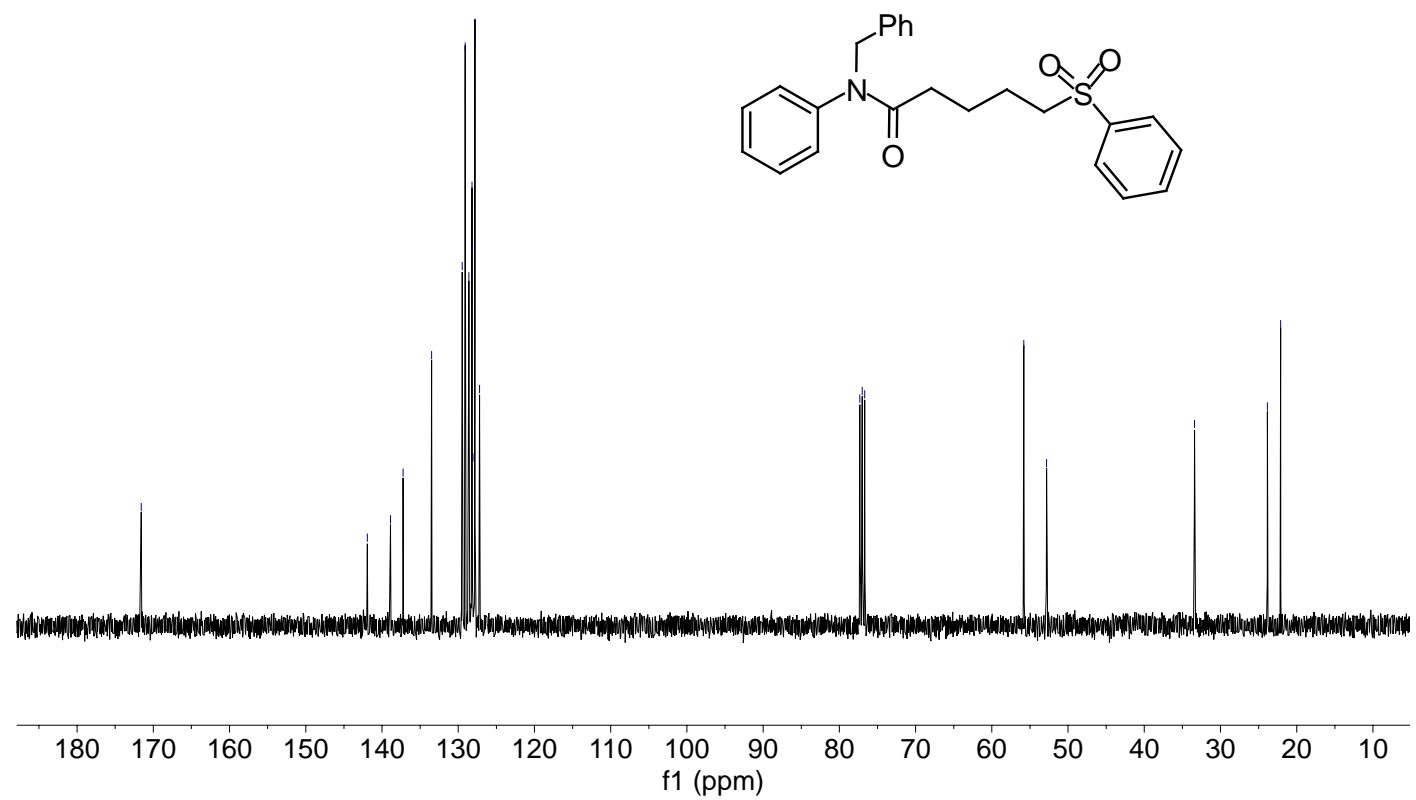




\section{$N$-allyl- $N$-phenyl-5-(phenylsulfonyl)pentanamide (3l)}

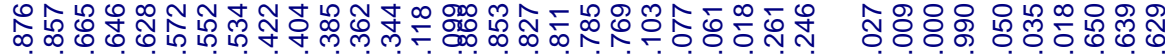

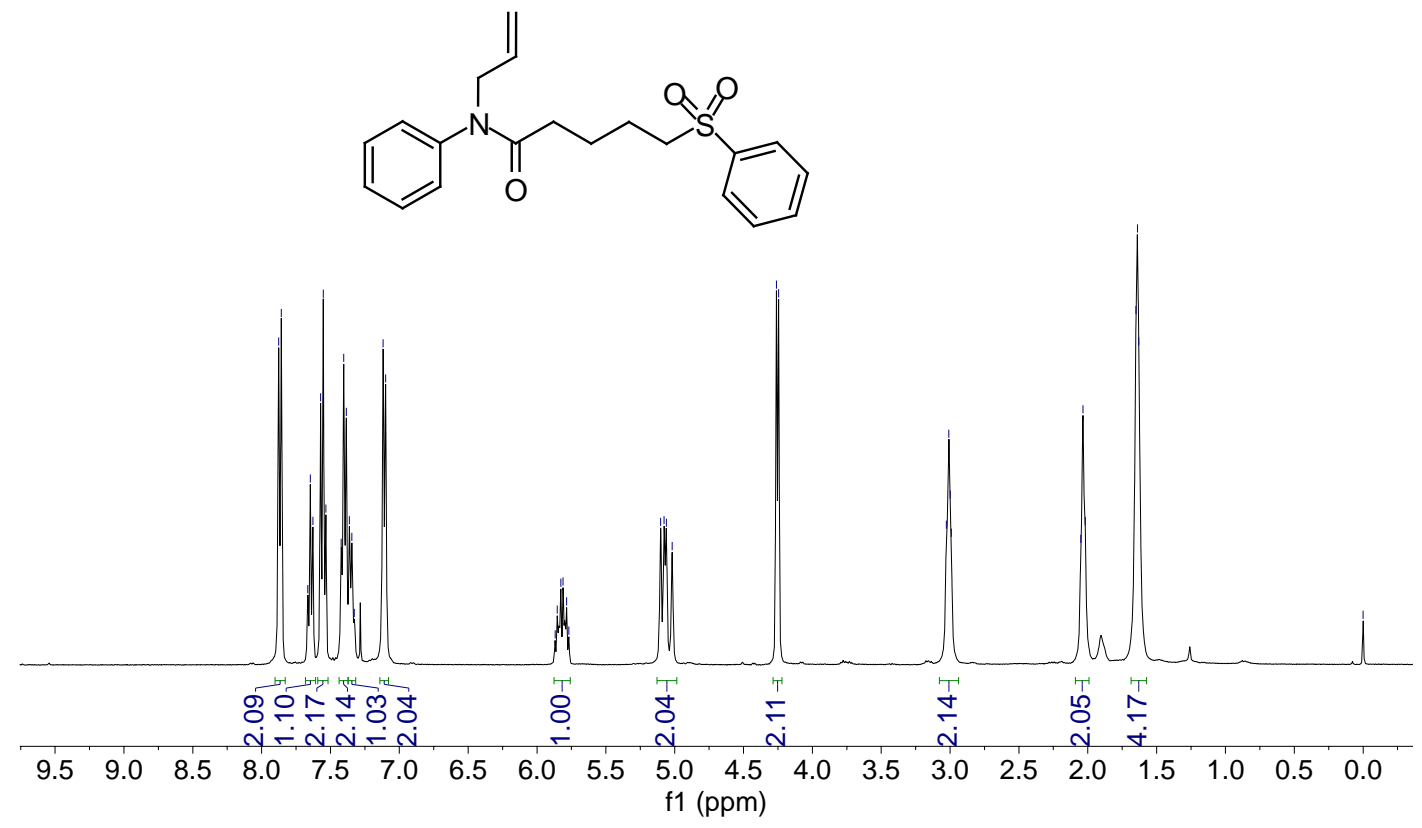<smiles>C=CCN(C(=O)CCCCS(=O)(=O)c1ccccc1)c1ccccc1</smiles> 
$N$-Phenyl- $N$-(3-(phenylsulfonyl)propyl)cyclopropanecarboxamide (3m)

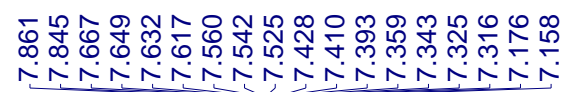

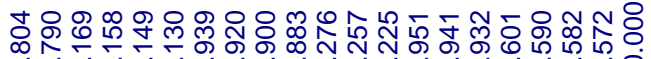

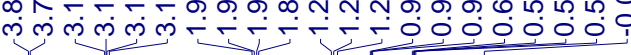<smiles>O=C(C1CC1)N(CCCS(=O)(=O)c1ccccc1)c1ccccc1</smiles>

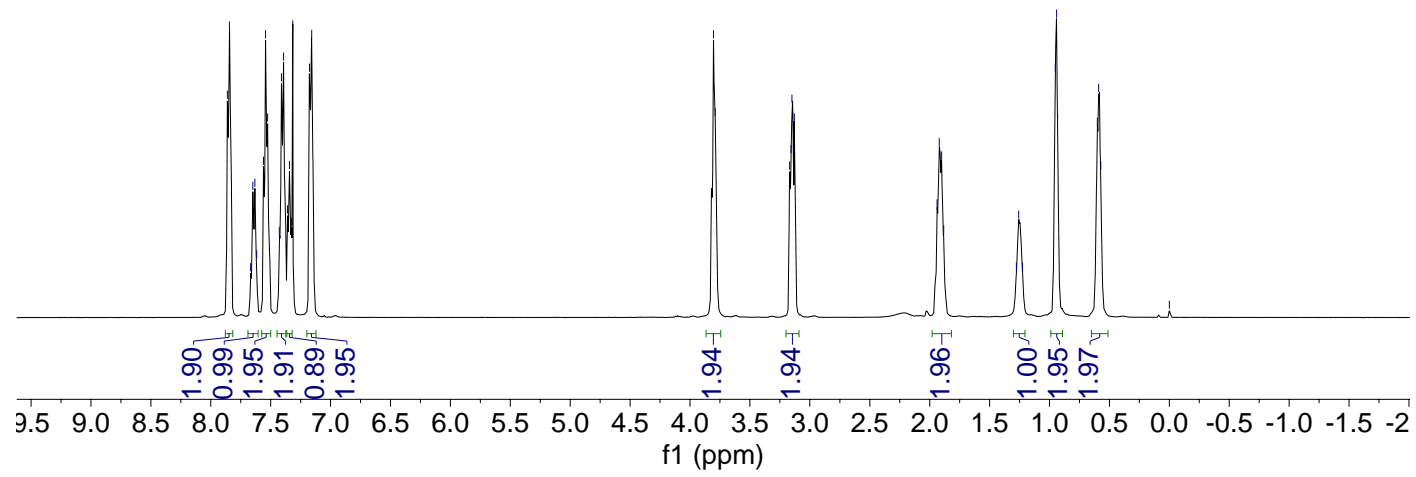

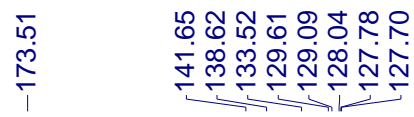

लํㅇㅇㅛ

रํํㅇ

$\bar{\sigma}$

กิ่

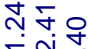

둥

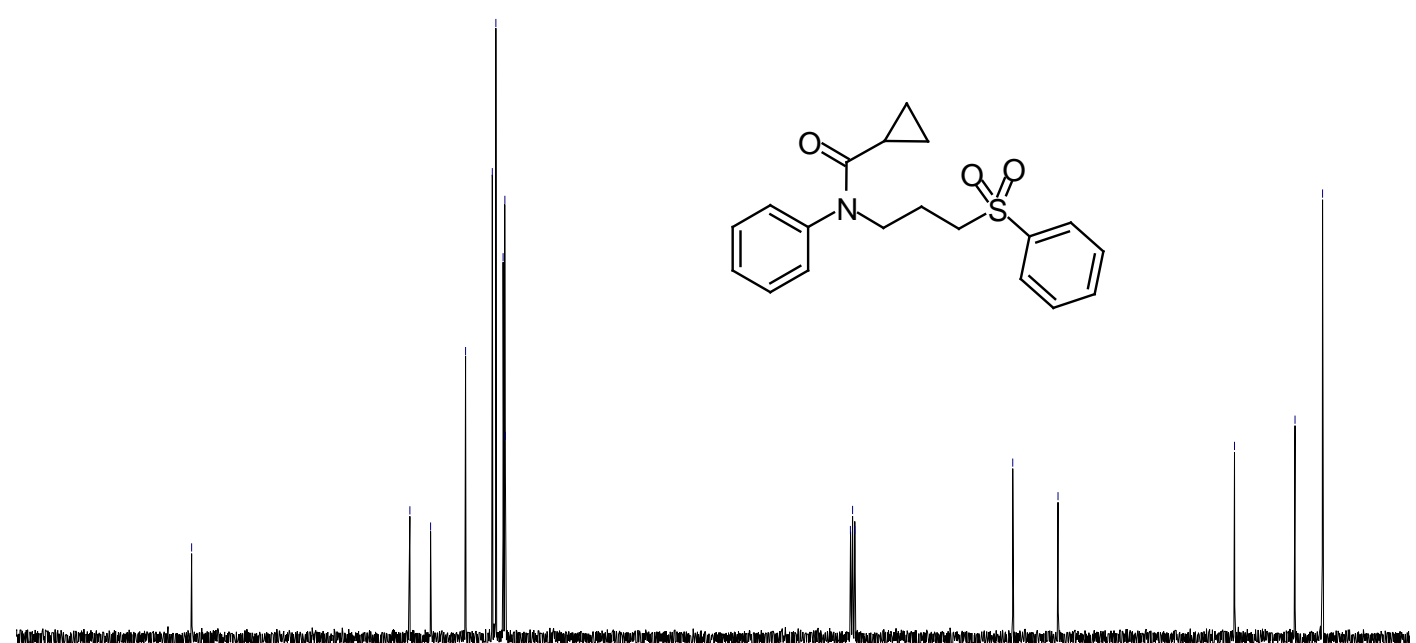

$\begin{array}{llllllllllllllllllll}190 & 180 & 170 & 160 & 150 & 140 & 130 & 120 & 110 & 100 & 90 & 80 & 70 & 60 & 50 & 40 & 30 & 20 & 10 & 0\end{array}$ f1 (ppm) 


\section{3-(Phenylsulfonyl)propyl $o$-tolylcarbamate (3n)}

때

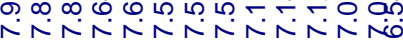

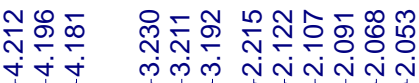

\&<smiles>Cc1ccccc1NC(=O)OCCCS(=O)(=O)c1ccccc1</smiles>

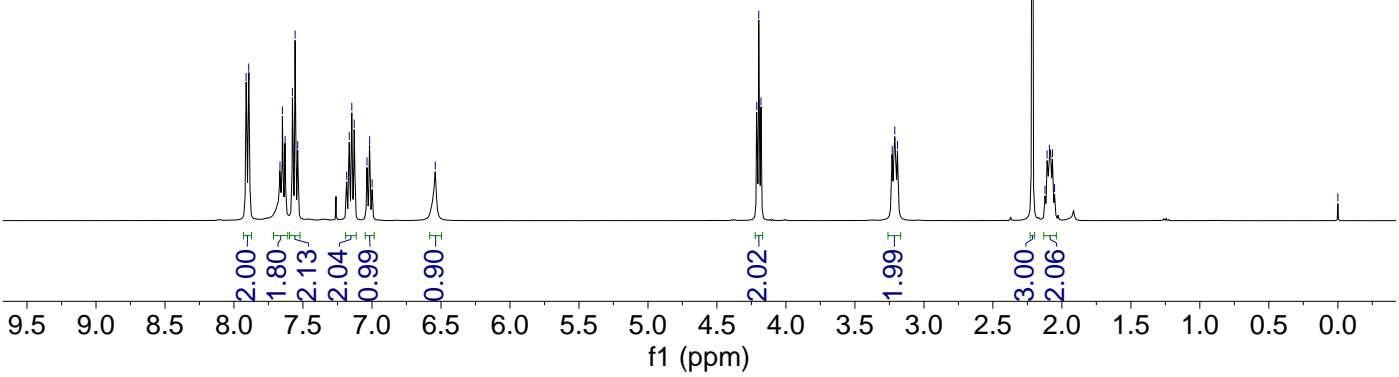

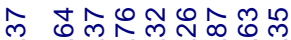

लिं

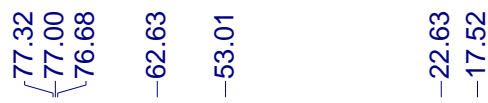

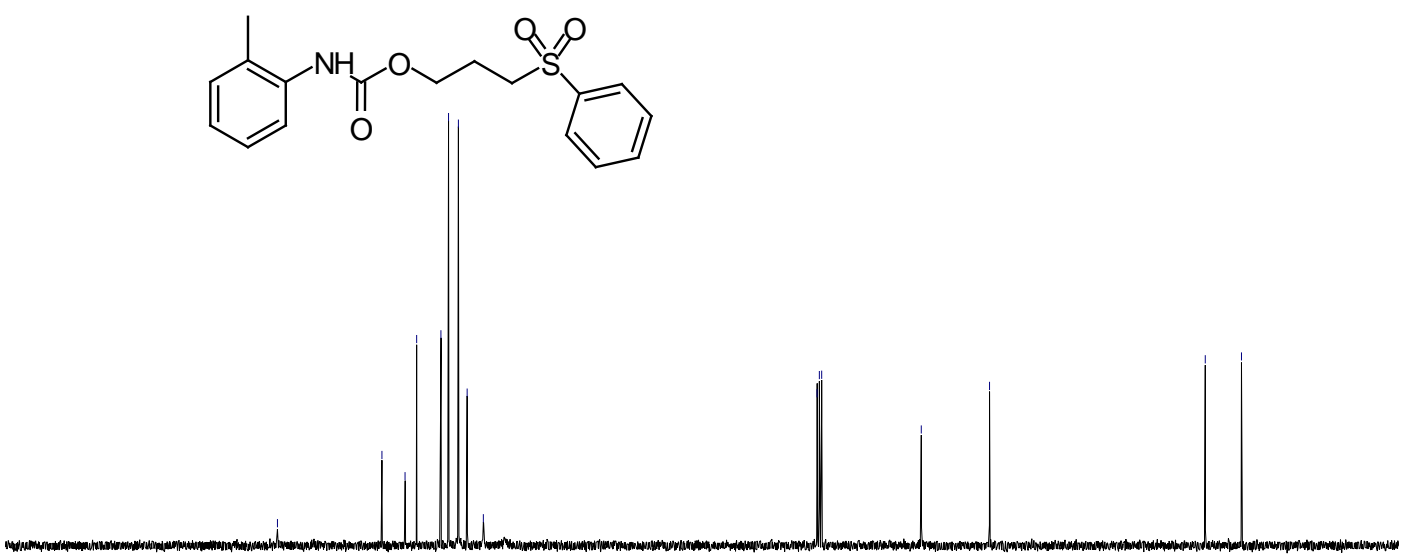

$\begin{array}{lllllllllll}90 & 180 & 170 & 160 & 150 & 140 & 130 & 120 & 110 & 100 & 90\end{array}$

$\begin{array}{lllllllll}80 & 70 & 60 & 50 & 40 & 30 & 20 & 10 & 0\end{array}$

(ppm) 


\section{5-(Phenylsulfonyl)- $N$-tosylpentanamide (3o)}
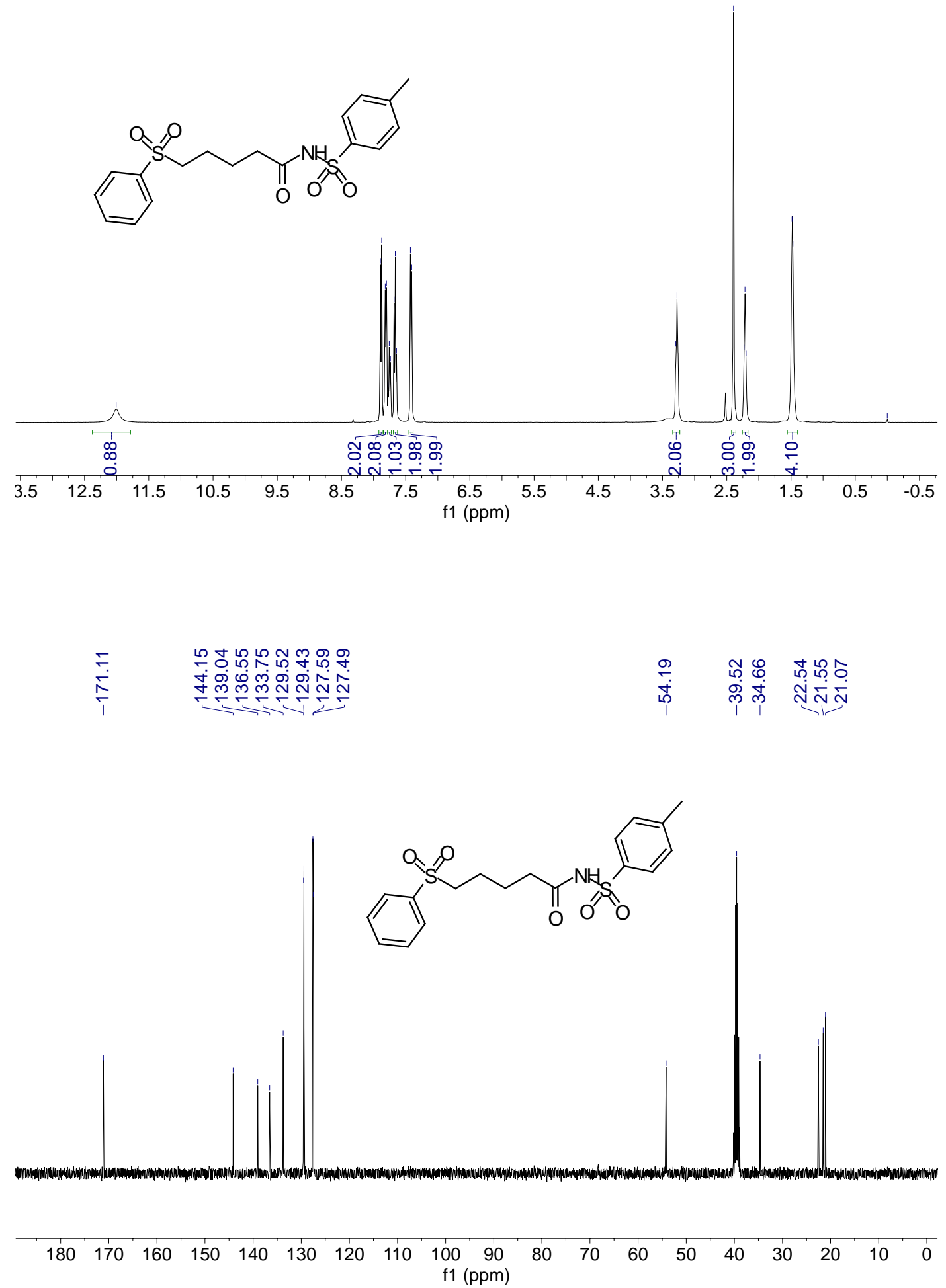
4-Methyl- $N$-(5-(phenylsulfonyl)pentyl)benzenesulfonamide (3p)

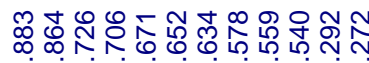

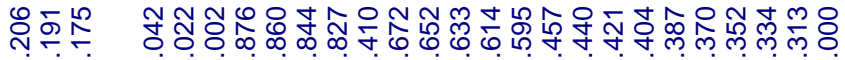
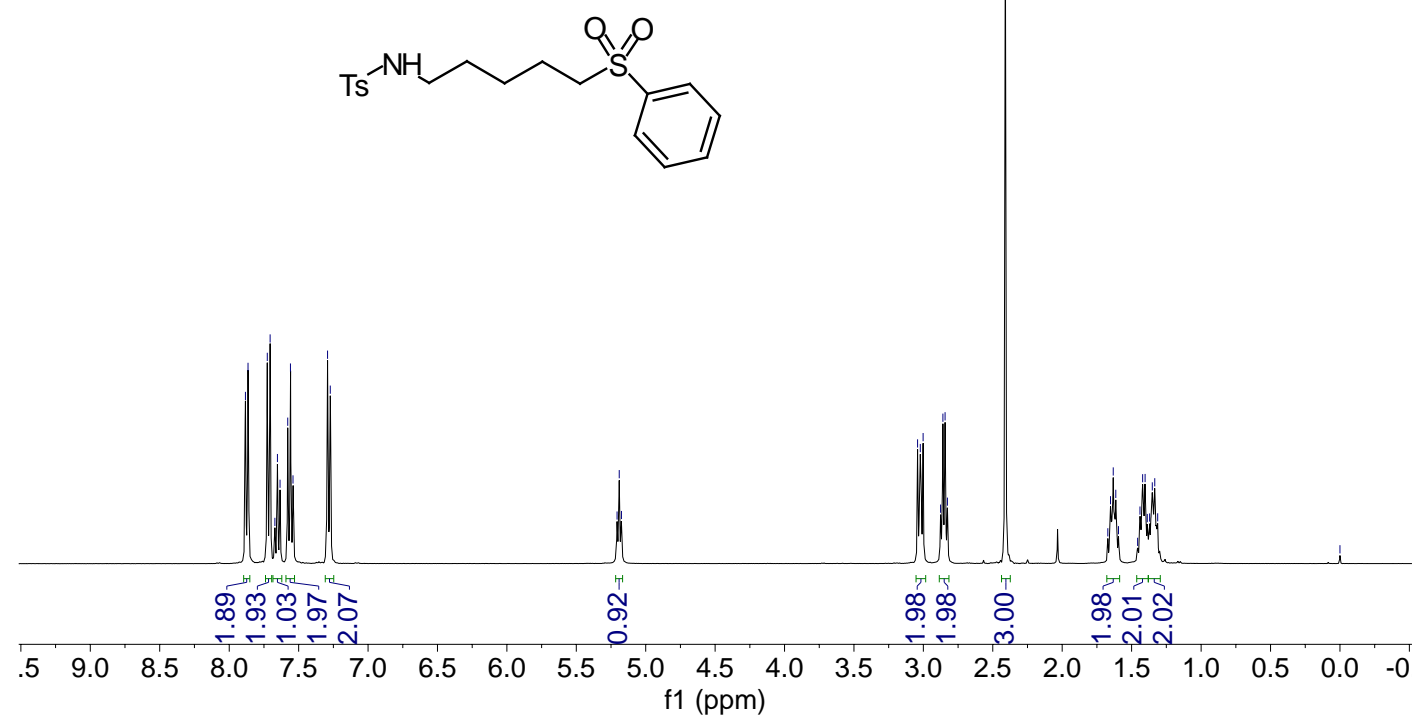

赵

守品

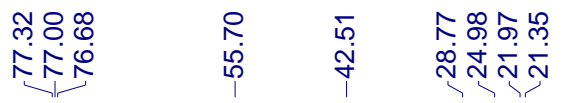

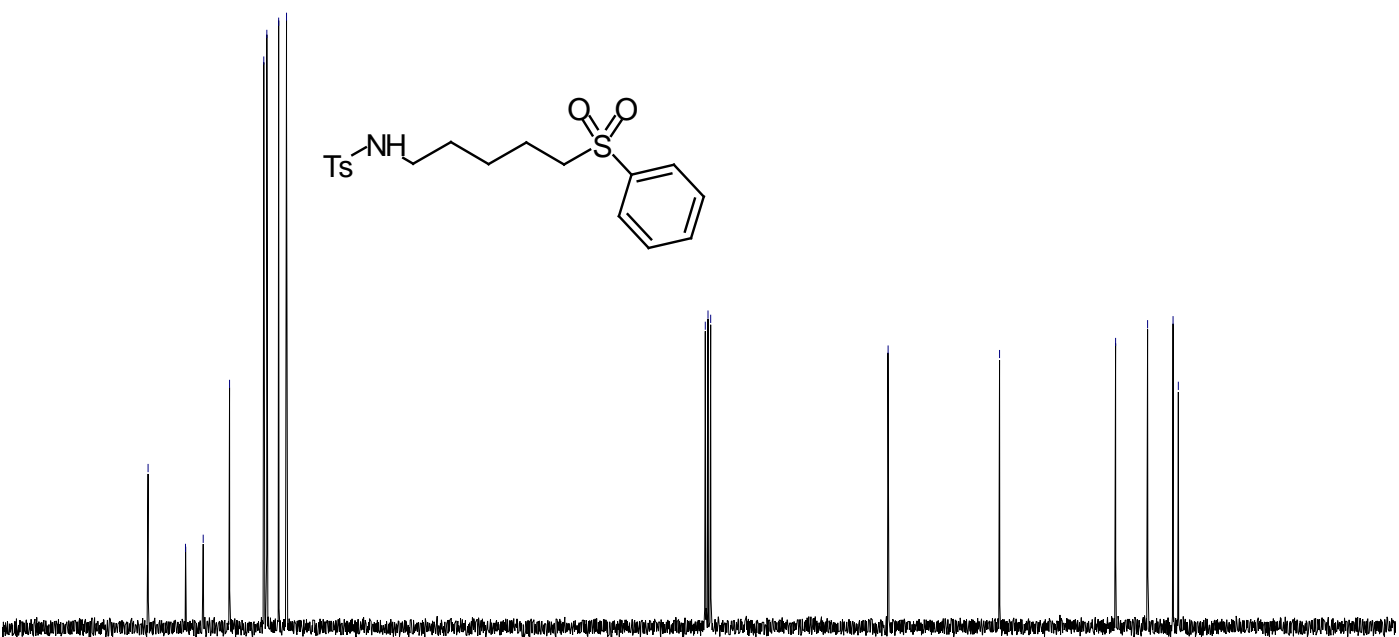

$\begin{array}{lllllllllllllllll}60 & 150 & 140 & 130 & 120 & 110 & 100 & 90 & \begin{array}{c}80 \\ \mathrm{f} 1(\mathrm{ppm})\end{array} & 70 & 60 & 50 & 40 & 30 & 20 & 10 & 0\end{array}$ 


\section{2-(5-(Phenylsulfonyl)pentyl)isoindoline-1,3-dione (3q)}

유잉 Niñ

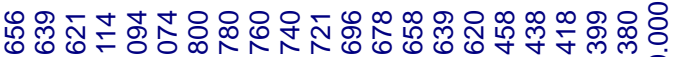

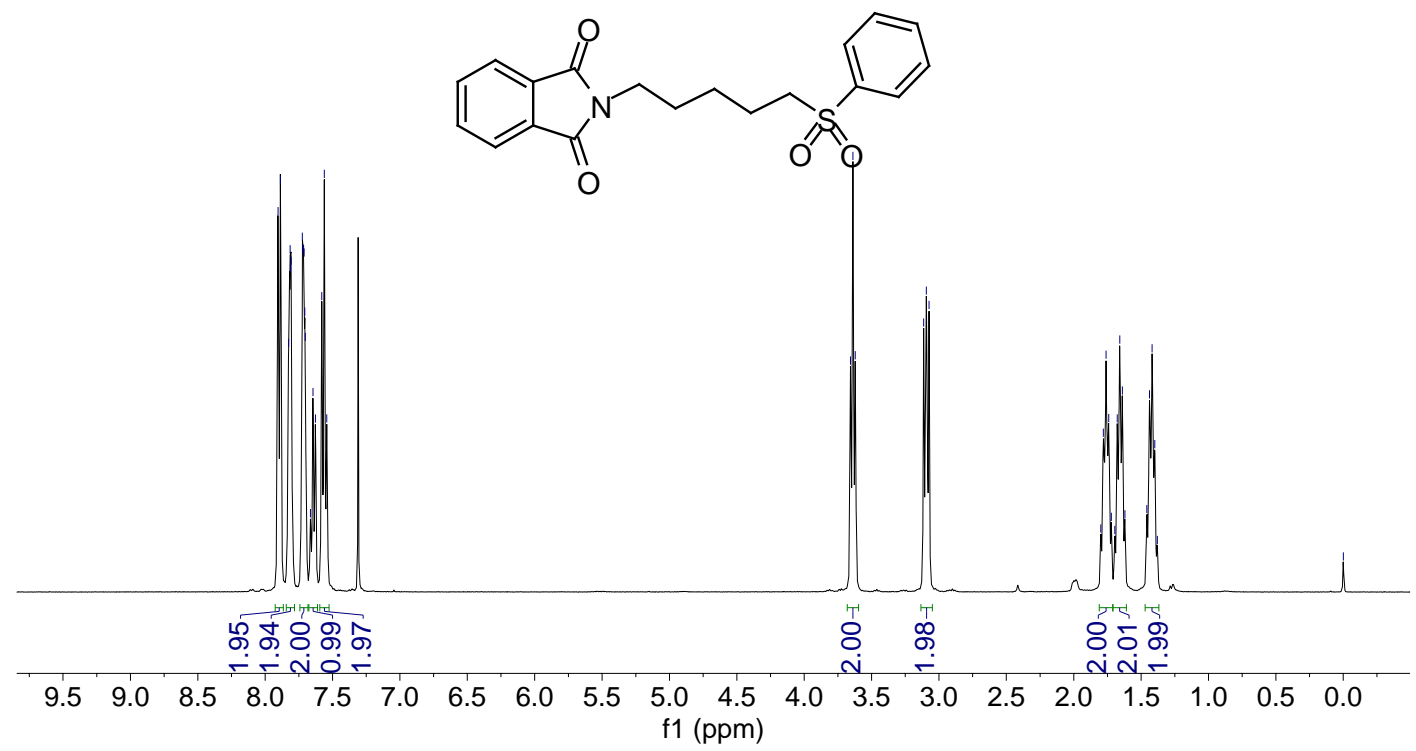

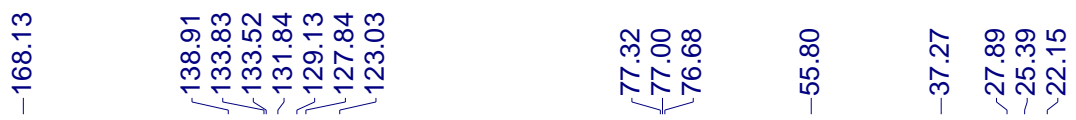

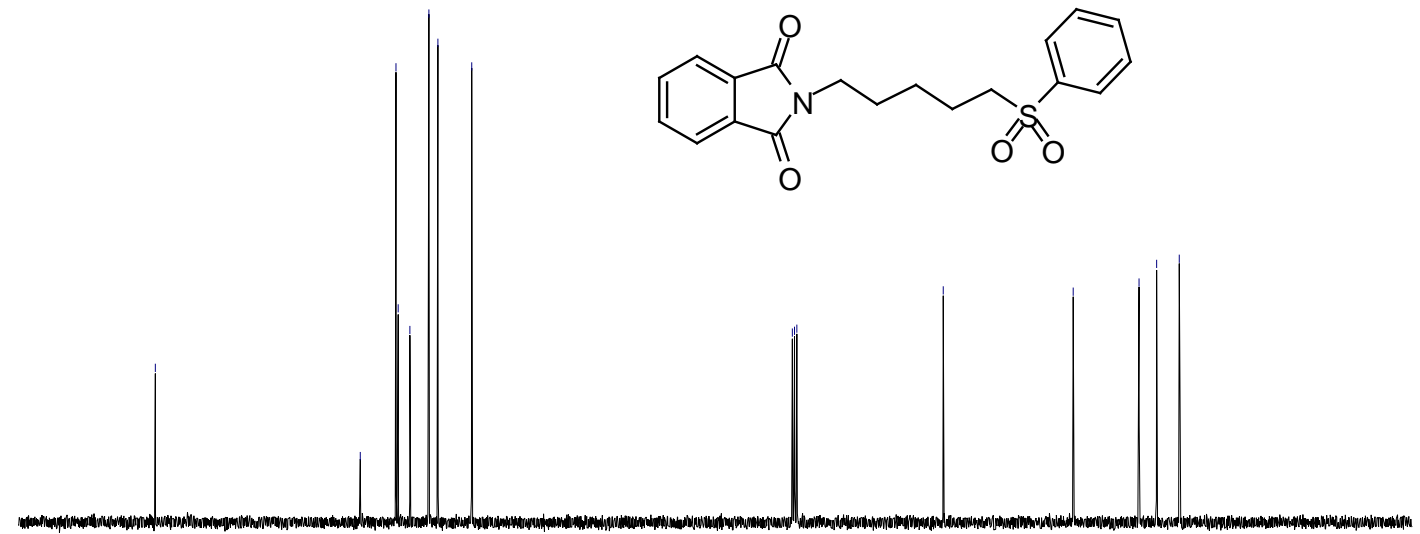

$\begin{array}{llllllllllllllllllll}180 & 170 & 160 & 150 & 140 & 130 & 120 & 110 & 100 & 90 & 80 & 70 & 60 & 50 & 40 & 30 & 20 & 10 & 0 & -1\end{array}$ f1 (ppm) 


\section{1-(Indolin-1-yl)-11-(phenylsulfonyl)undecan-1-one (3r)}

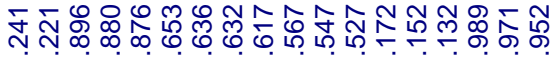
on
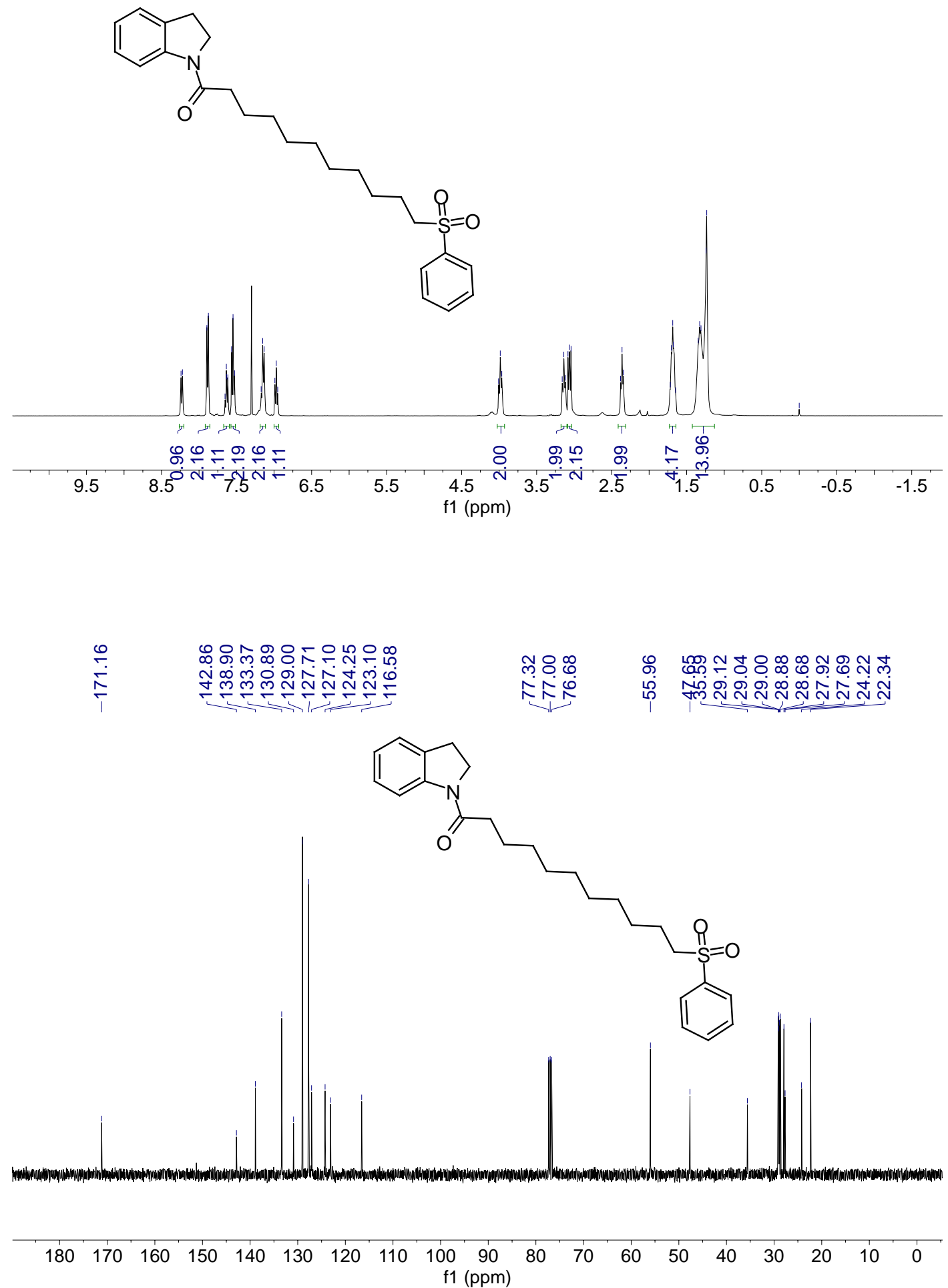


\section{4-(Phenylsulfonyl)butyl furan-2-carboxylate (3s)}

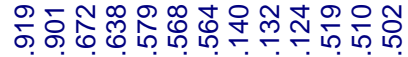

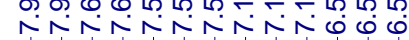

品芦

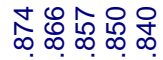
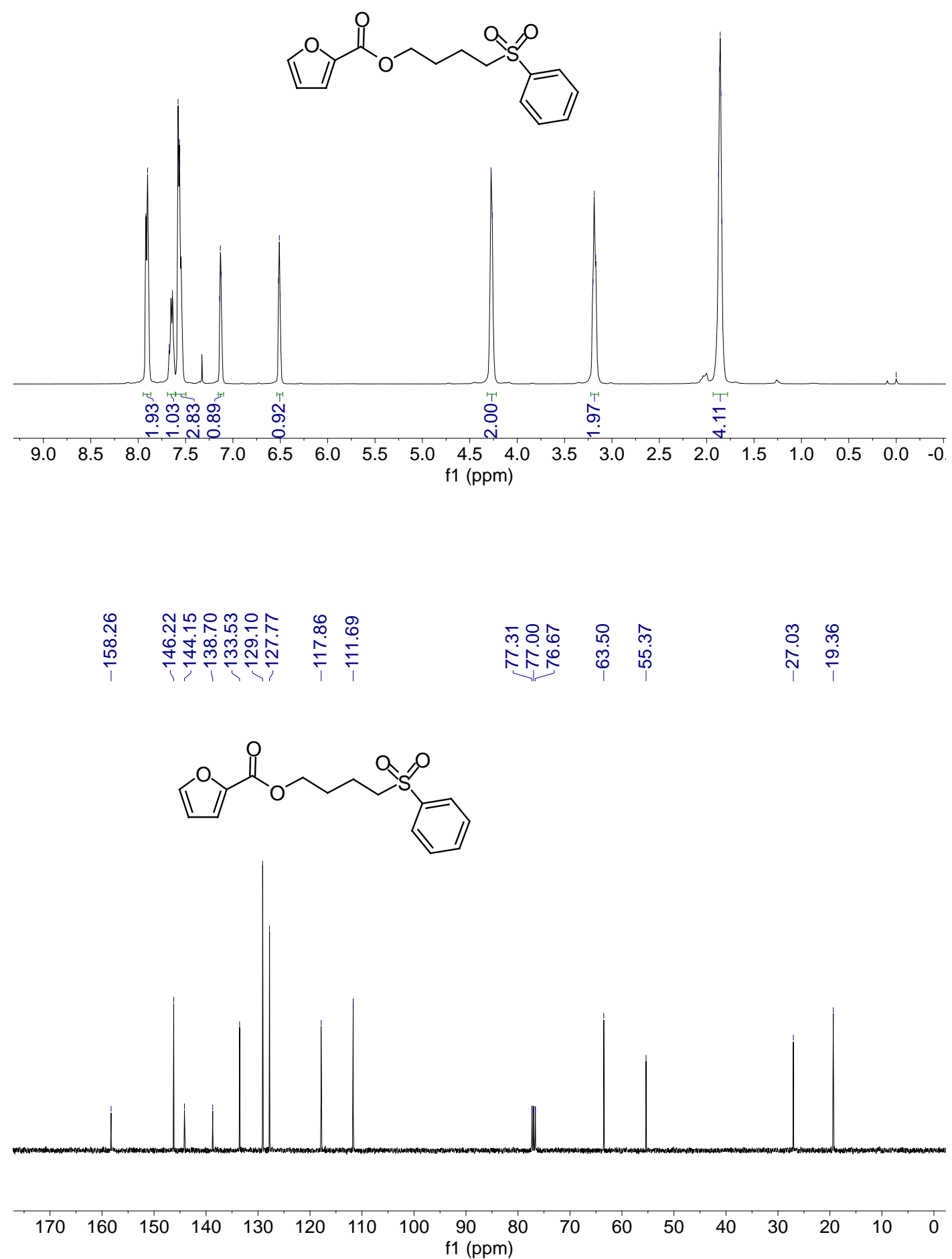


\section{5-(Phenylsulfonyl)pentyl benzoate (3t)}

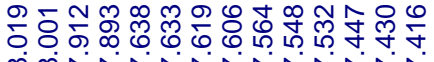

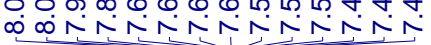

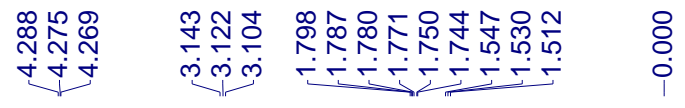<smiles>O=C(OCCCCCS(=O)(=O)c1ccccc1)c1ccccc1</smiles>

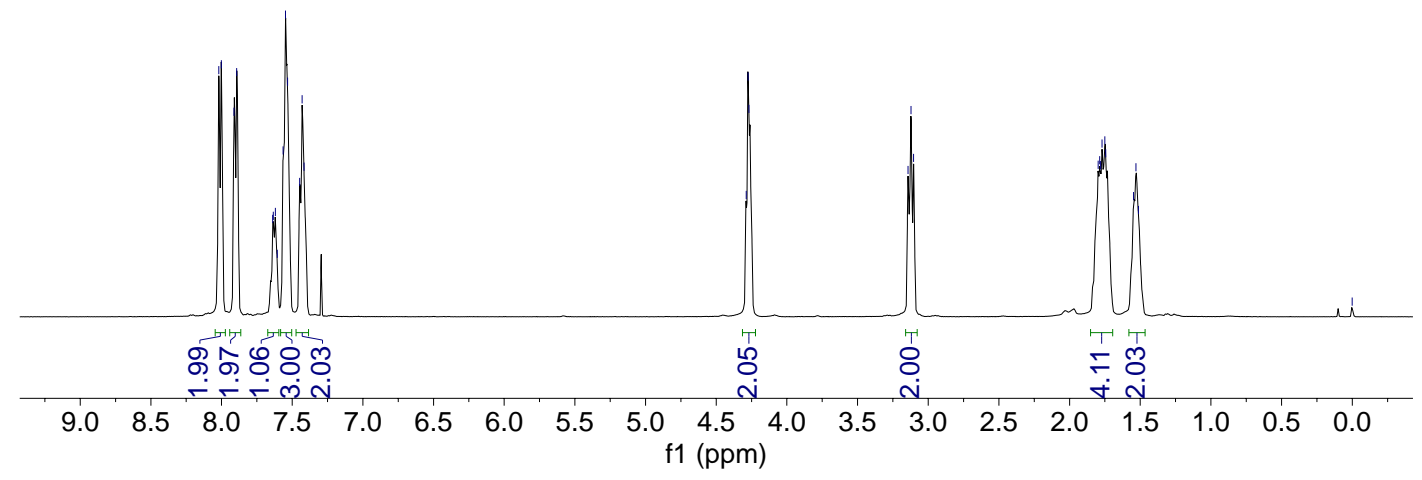

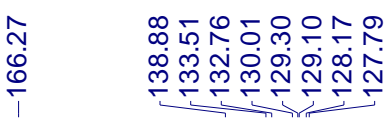

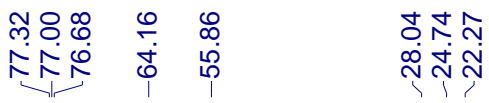

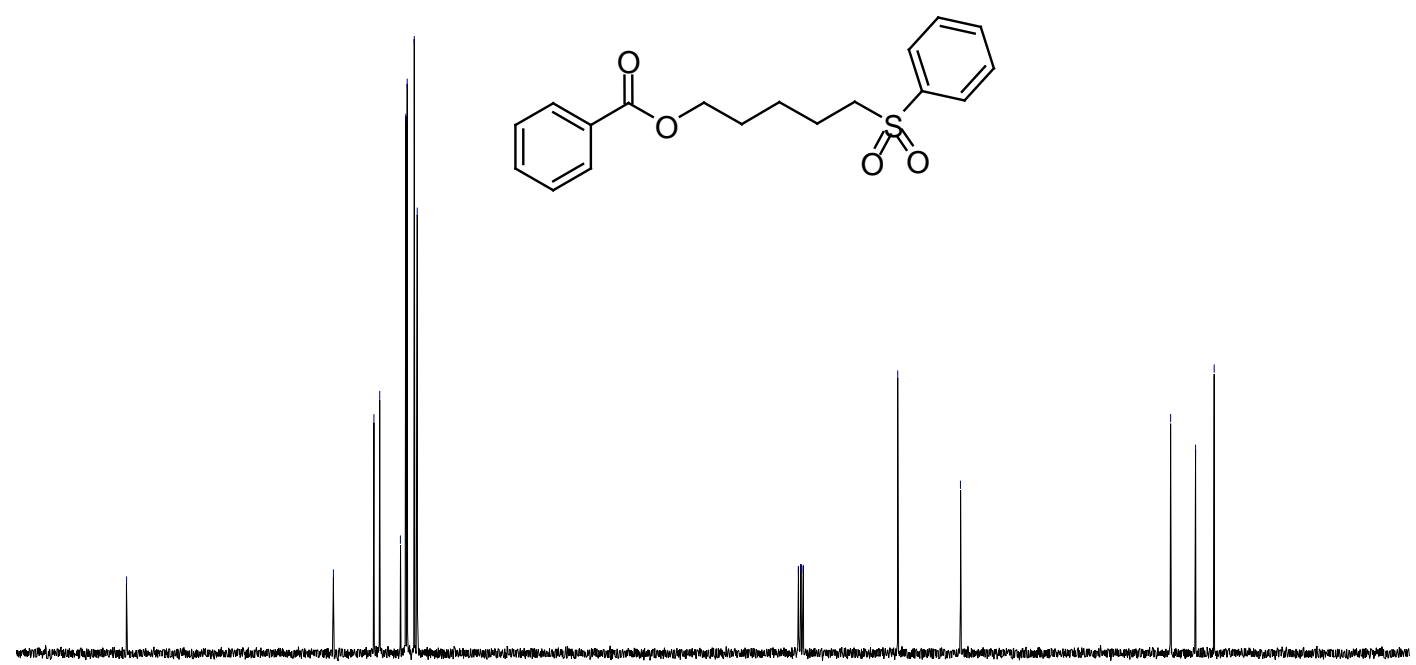

$\begin{array}{lllllllllllllllllllll}80 & 170 & 160 & 150 & 140 & 130 & 120 & 110 & 100 & \begin{array}{c}90 \\ \mathrm{f} 1(\mathrm{ppm})\end{array} & 80 & 70 & 60 & 50 & 40 & 30 & 20 & 10 & 0\end{array}$ 


\section{3-(Phenylsulfonyl)propyl benzoate (3u)}

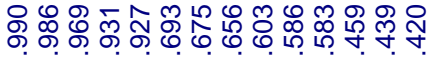

NNNNNNNNNNNN

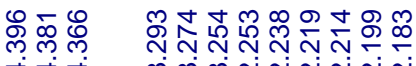<smiles>O=C(OCCCS(=O)(=O)c1ccccc1)c1ccccc1</smiles>

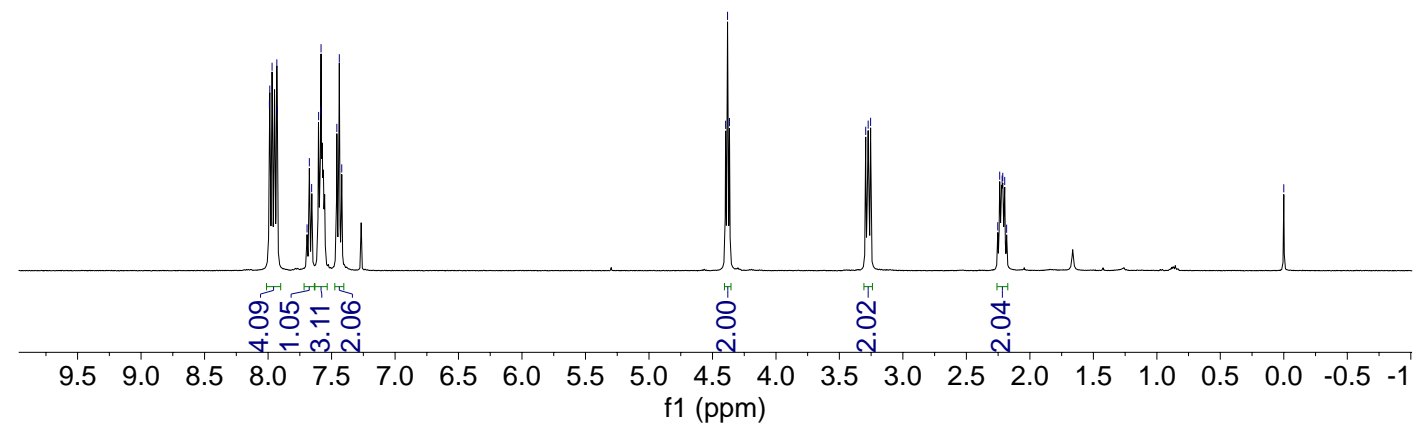

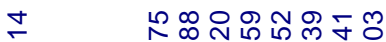

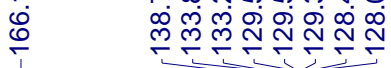

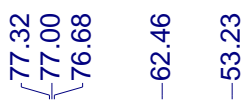

ลิ<smiles>O=C(OCCCS(=O)(=O)c1ccccc1)c1ccccc1</smiles>
$\begin{array}{llllllllll}170 & 160 & 150 & 140 & 130 & 120 & 110 & 100 & \begin{array}{l}90 \\ \mathrm{f} 1(\mathrm{ppm})\end{array}\end{array}$ 


\section{4-(Phenylsulfonyl)butyl 1-methyl-1H-pyrrole-2-carboxylate (3v)}

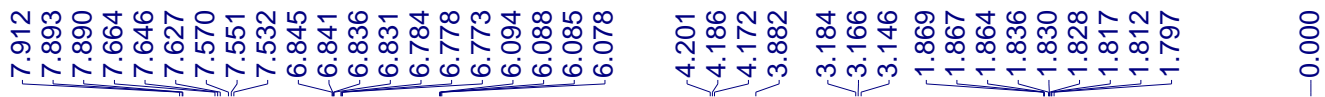

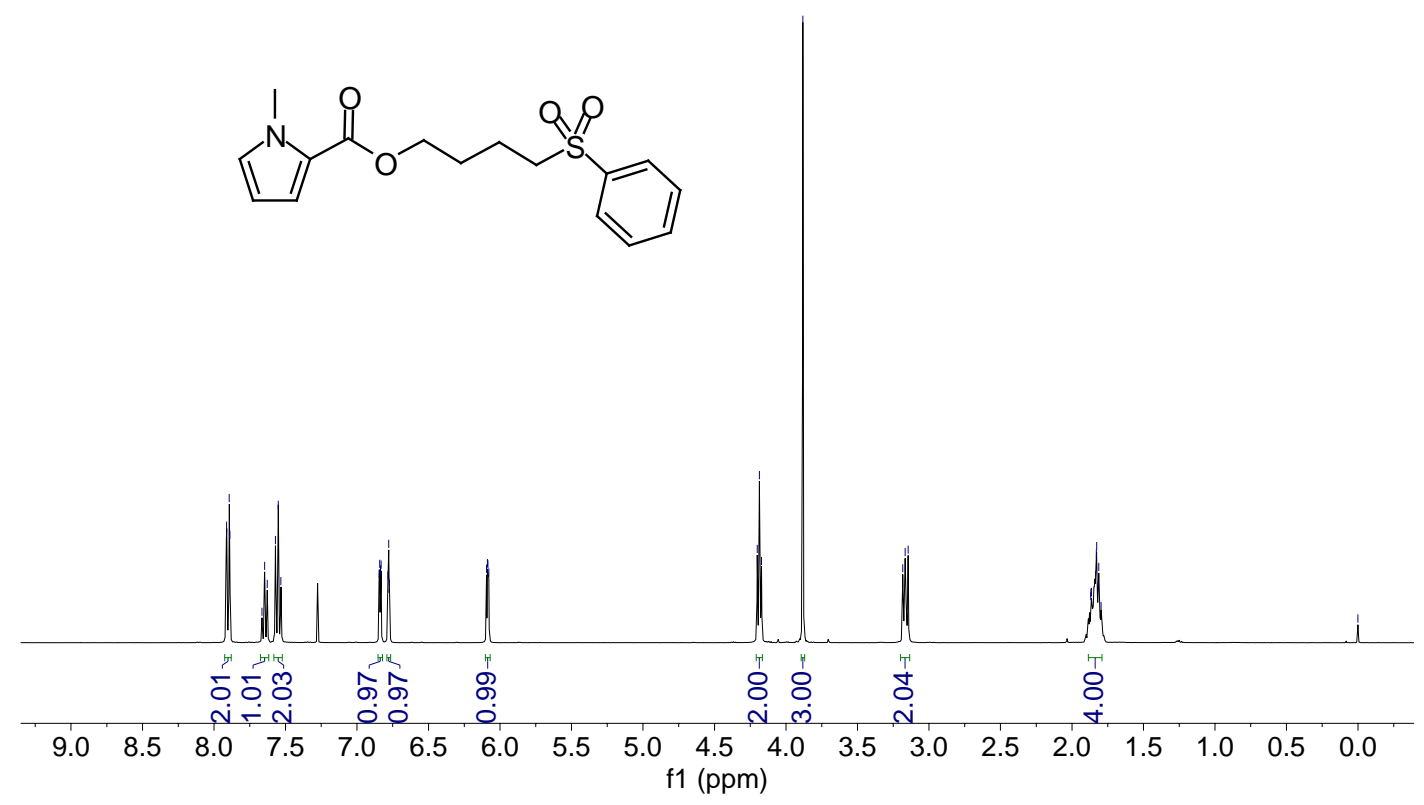

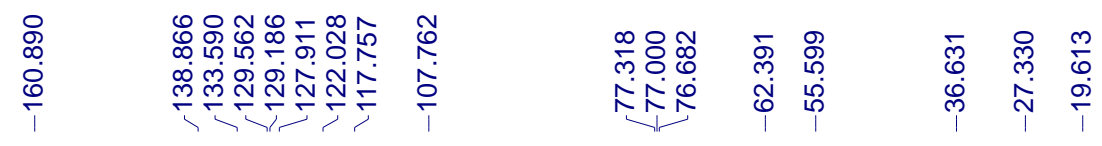

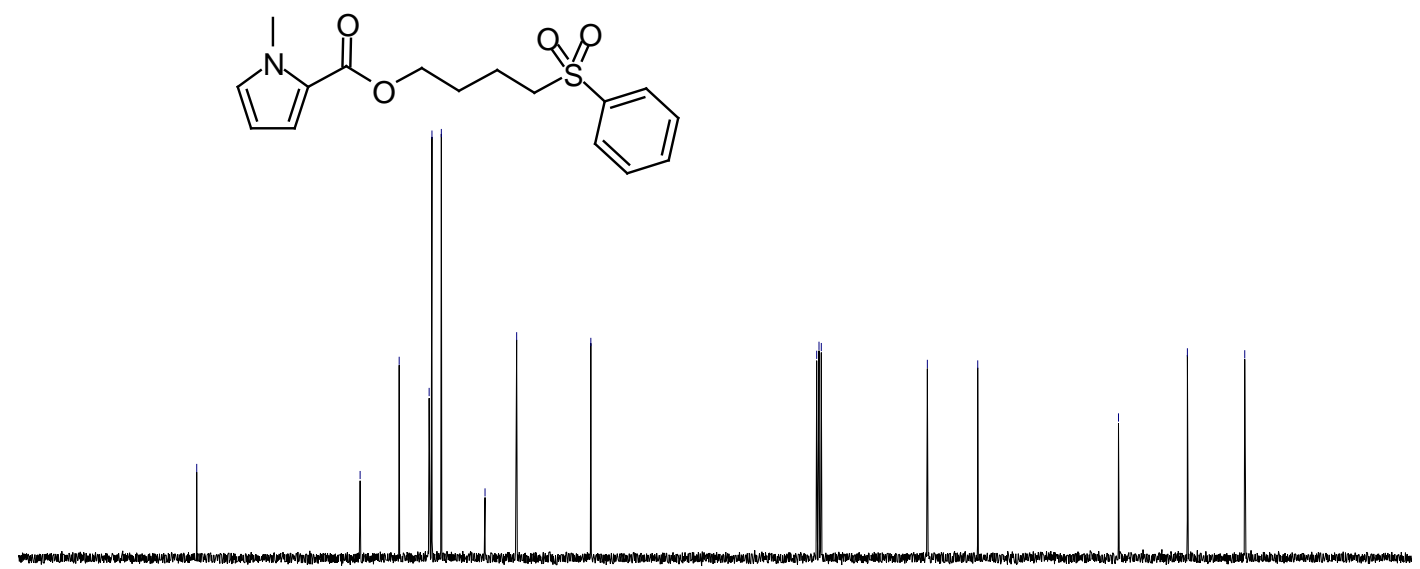

$\begin{array}{lllllllllllllllllll}180 & 170 & 160 & 150 & 140 & 130 & 120 & 110 & 100 & \begin{array}{c}90 \\ \mathrm{f} 1(\mathrm{ppm})\end{array} & 80 & 70 & 60 & 50 & 40 & 30 & 20 & 10 & 0\end{array}$ 
1-Phenyl-6-(phenylsulfonyl)hexan-1-one (3w)

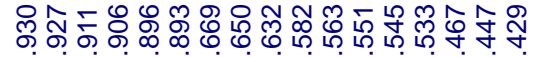

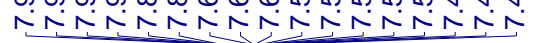

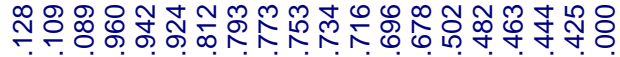

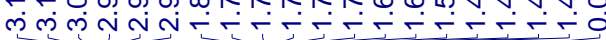

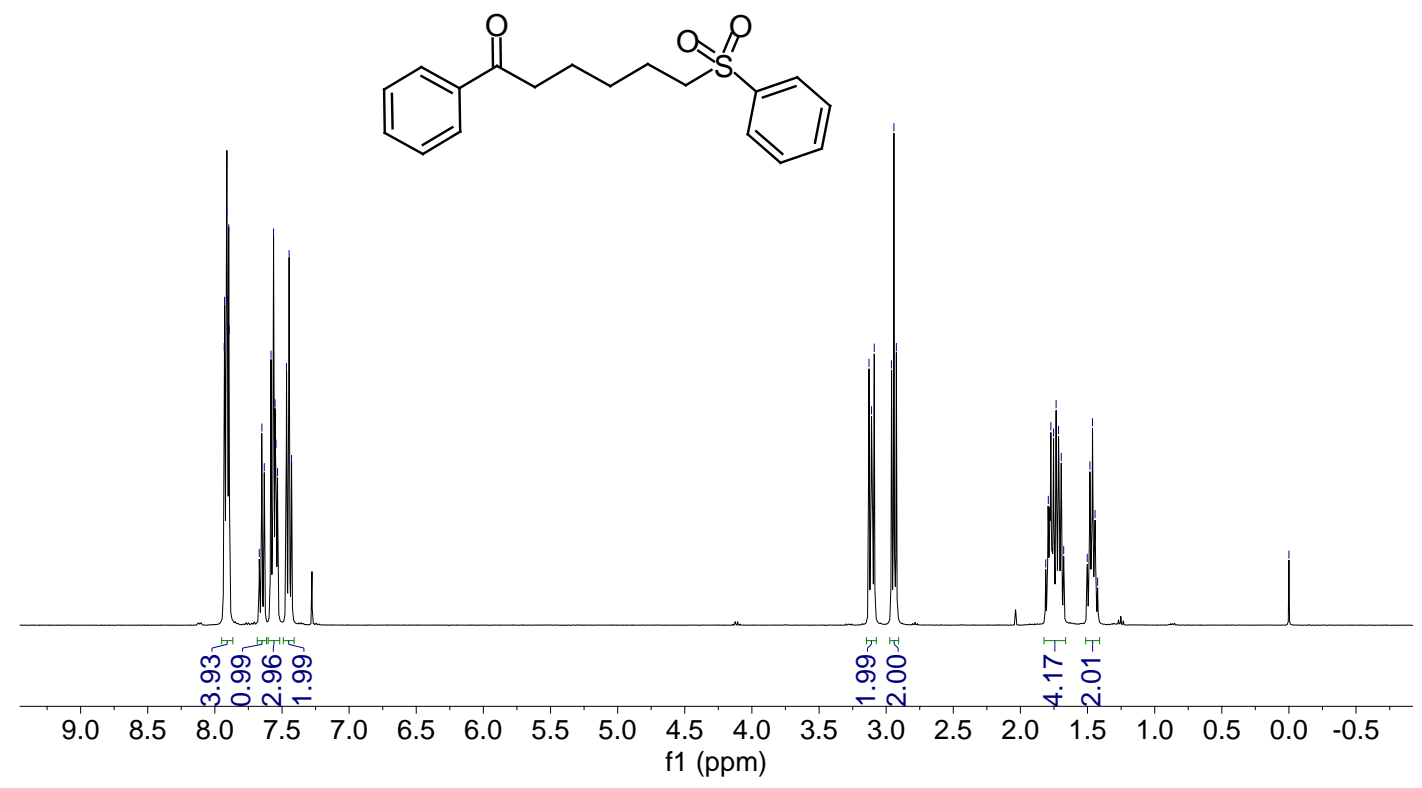

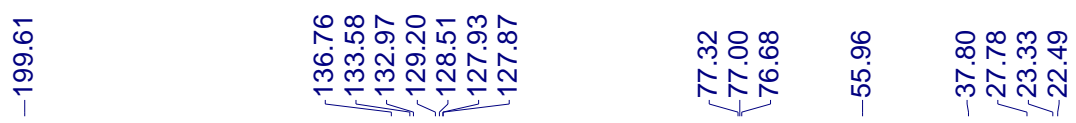

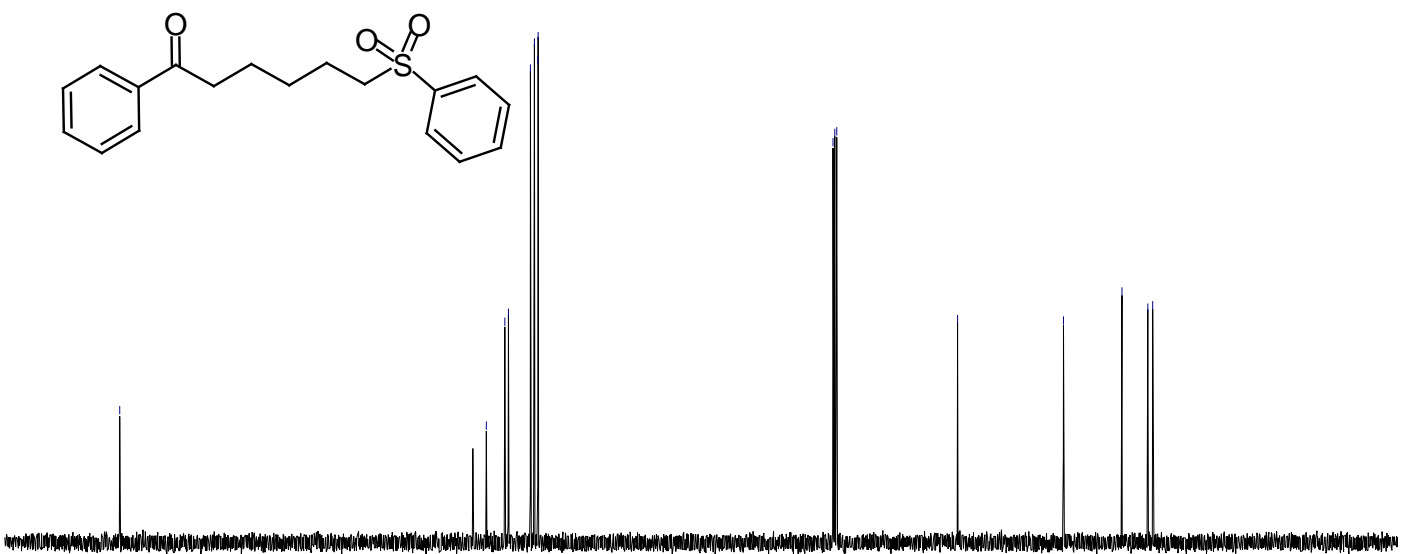

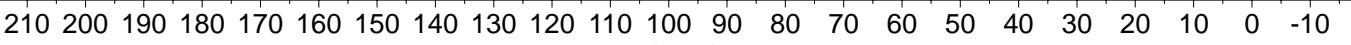
f1 (ppm) 


\section{5-(Phenylsulfonyl)pentyl 4-methylbenzenesulfonate (3x)}

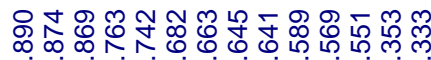

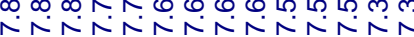

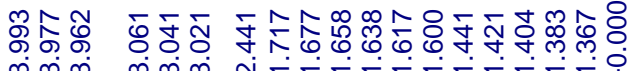

ल ल लm

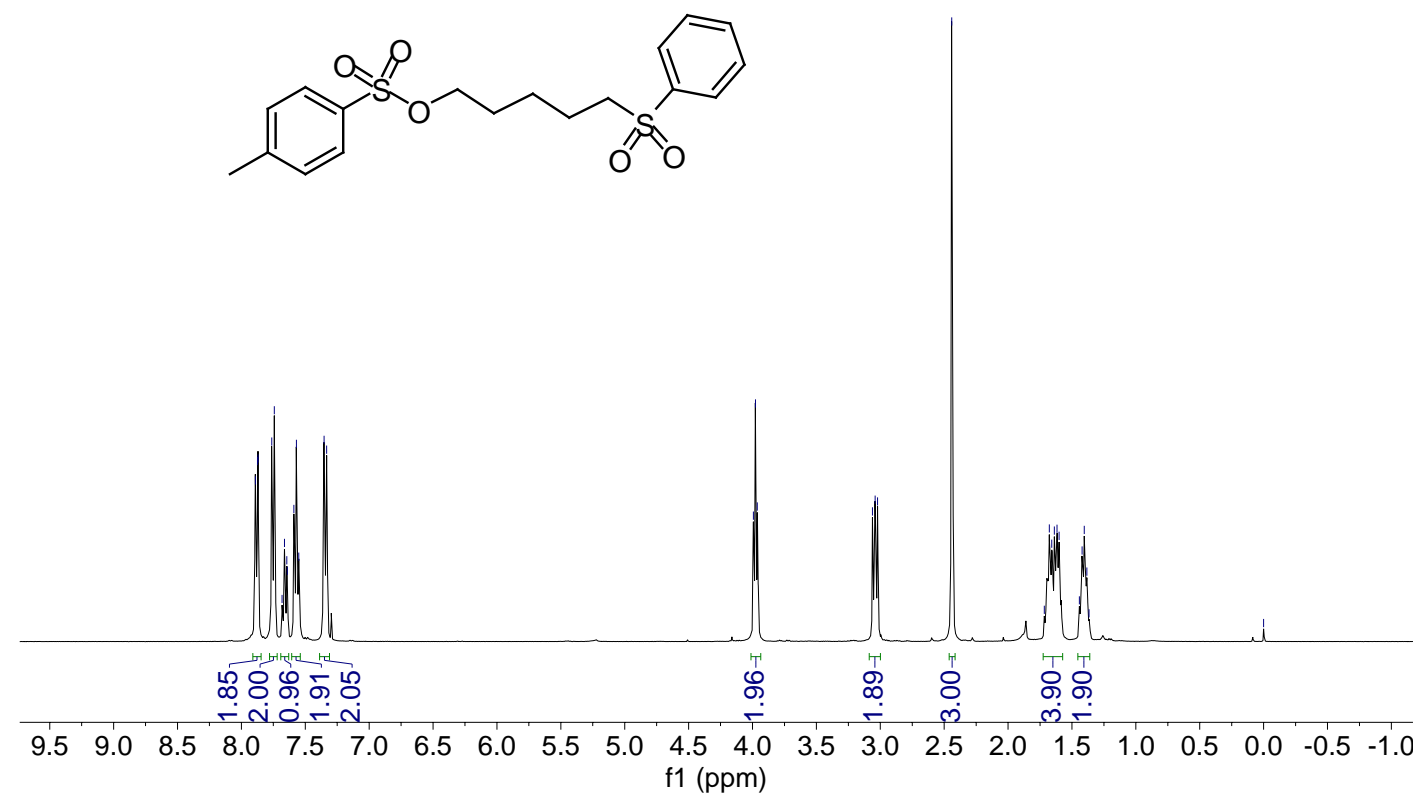

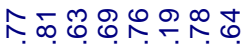

守品

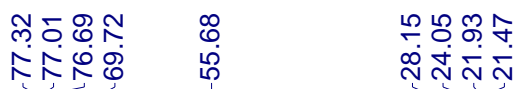<smiles>Cc1ccc(S(=O)(=O)OCCCCCS(=O)(=O)c2ccccc2)cc1</smiles>

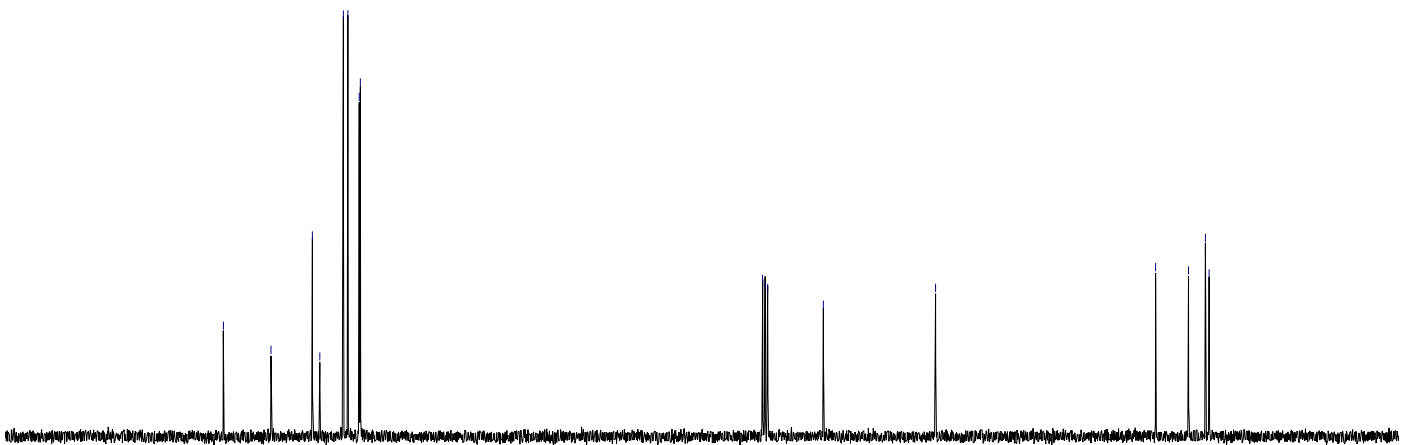

$\begin{array}{llllllllllllllllll}170 & 160 & 150 & 140 & 130 & 120 & 110 & 100 & \begin{array}{c}90 \\ \mathrm{f} 1(\mathrm{ppm})\end{array} & 70 & 60 & 50 & 40 & 30 & 20 & 10 & 0\end{array}$ 


\section{((4-Phenoxybutyl)sulfonyl)benzene (3y)}
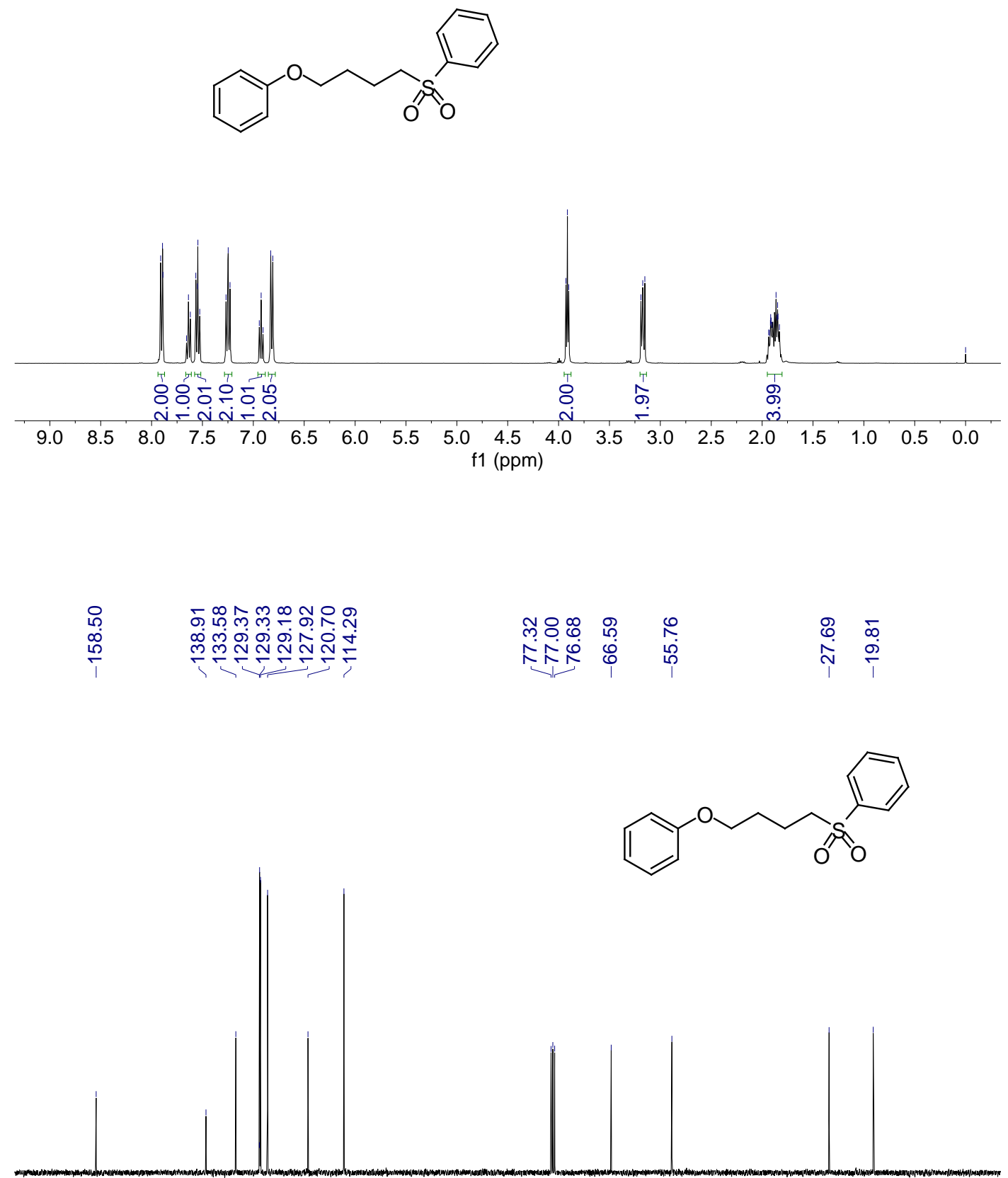

$\begin{array}{llllllllllllllllll}170 & 160 & 150 & 140 & 130 & 120 & 110 & 100 & \begin{array}{c}90 \\ \mathrm{f} 1(\mathrm{ppm})\end{array} & 70 & 60 & 50 & 40 & 30 & 20 & 10 & 0\end{array}$ 


\section{5-(Phenylsulfonyl)pentanoic acid (3z)}

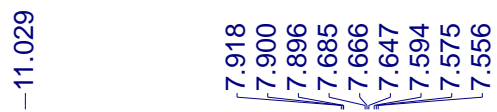

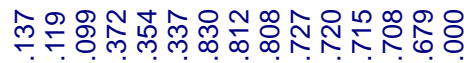
लं लं

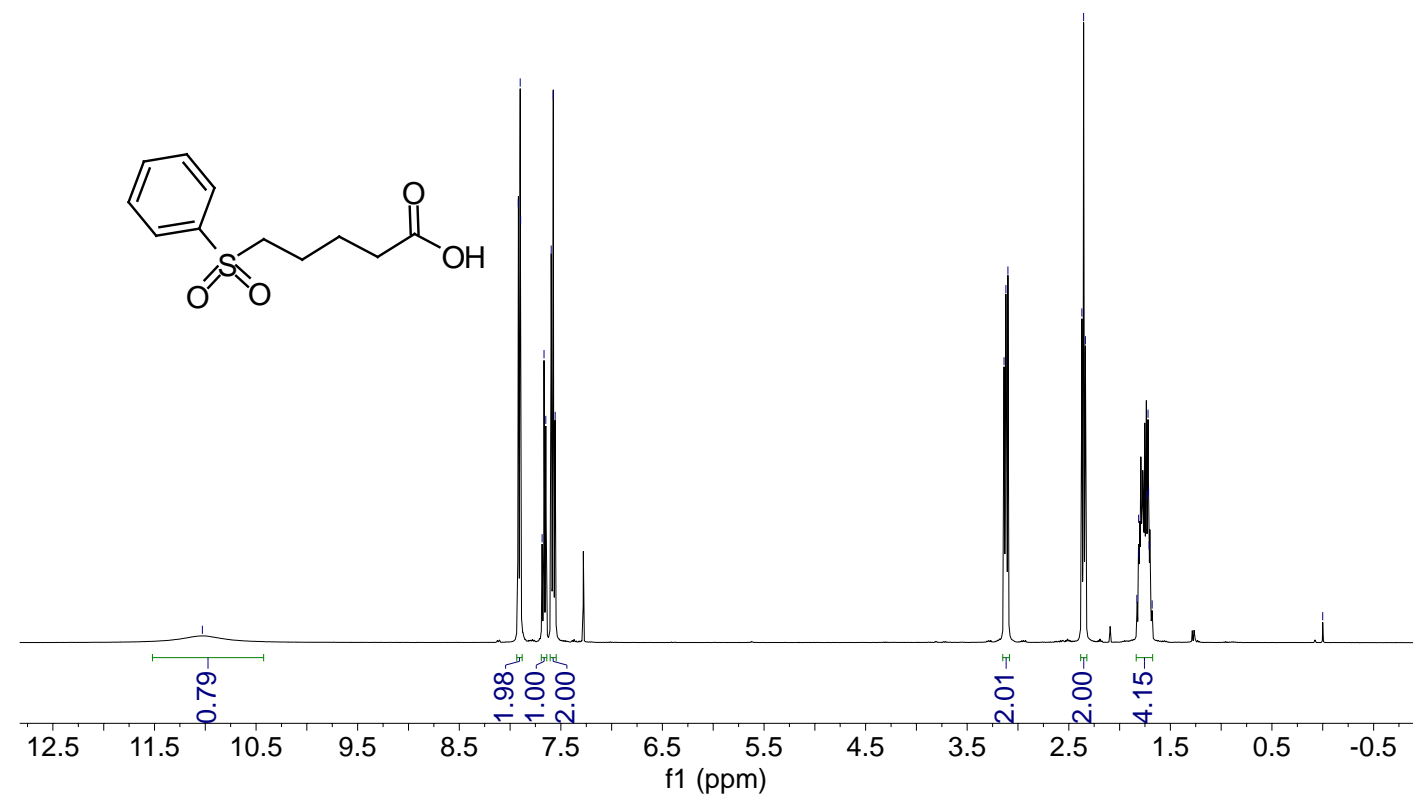

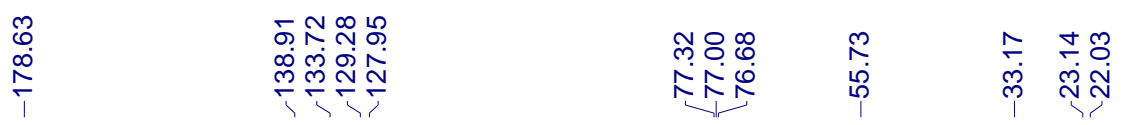

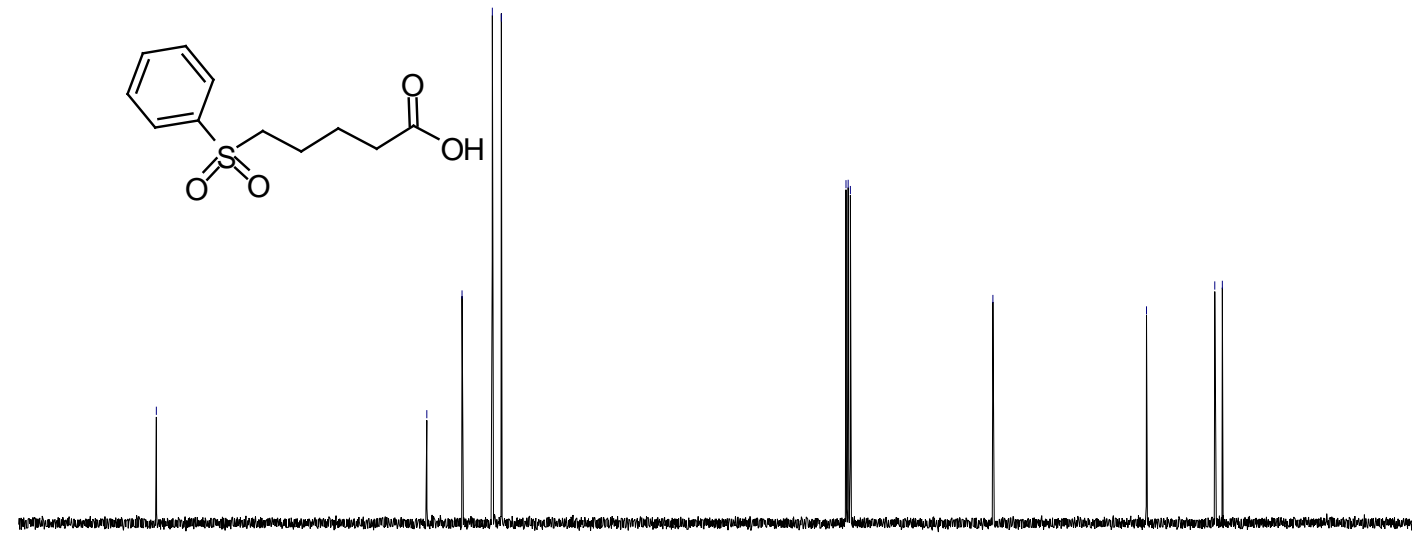

$\begin{array}{llllllllllllllllllll}190 & 180 & 170 & 160 & 150 & 140 & 130 & 120 & 110 & 100 & 90 & 80 & 70 & 60 & 50 & 40 & 30 & 20 & 10 & 0\end{array}$ f1 (ppm) 
7-(Phenylsulfonyl)heptanoic acid (3aa)
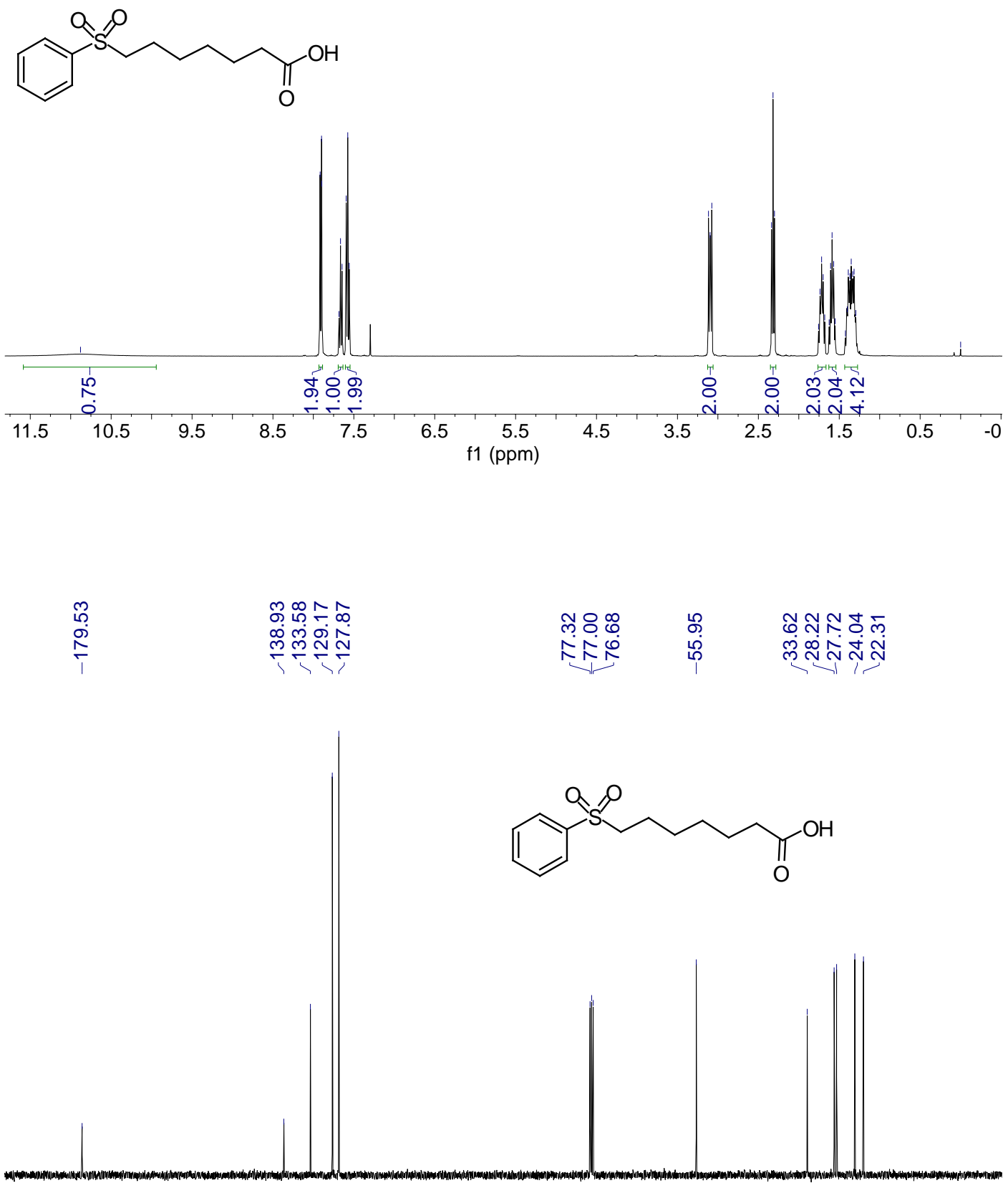

$\begin{array}{lllllllllll}190 & 180 & 170 & 160 & 150 & 140 & 130 & 120 & 110 & 100 & 90\end{array}$ f1 (ppm) 
<smiles>O=S(=O)(CCCCCCO)c1ccccc1</smiles>

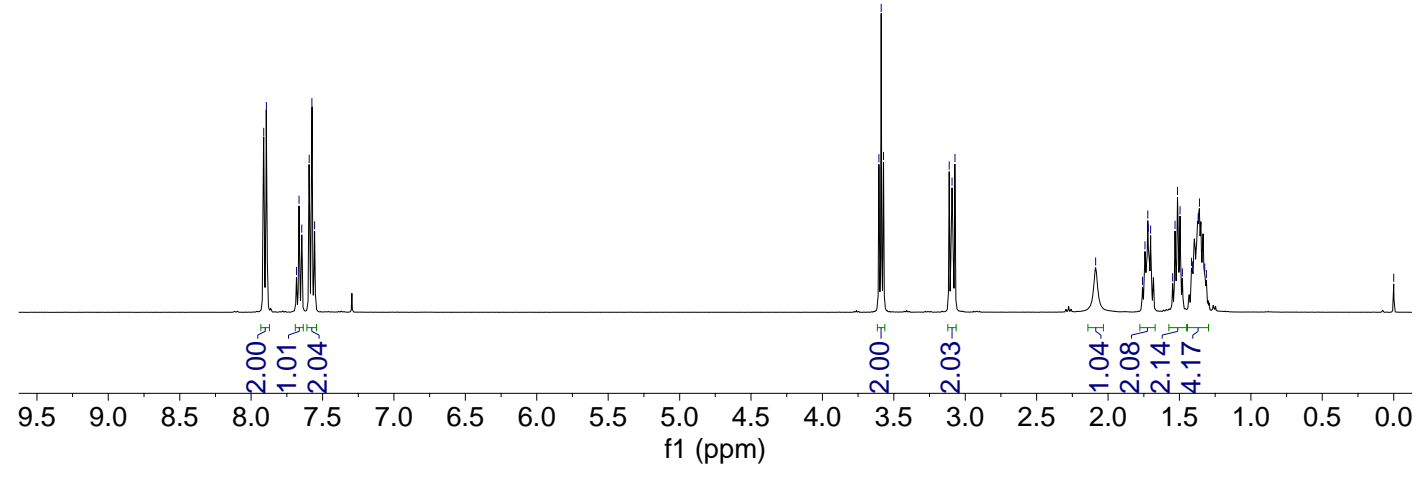

\section{8 웅요

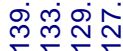

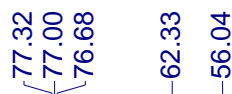

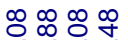

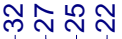

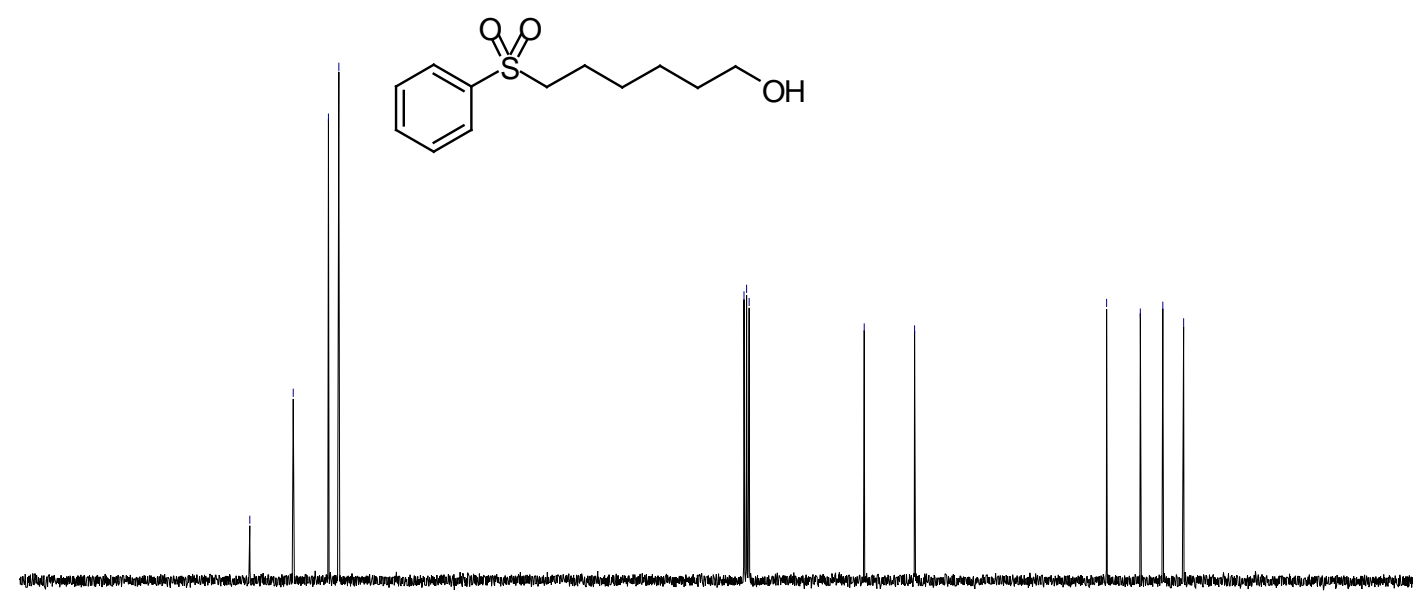

$\begin{array}{lllllllllllllllll}160 & 150 & 140 & 130 & 120 & 110 & 100 & 90 & \begin{array}{c}80 \\ \mathrm{f} 1(\mathrm{ppm})\end{array} & 70 & 60 & 50 & 40 & 30 & 20 & 10 & 0\end{array}$ 
((5-Bromopentyl)sulfonyl)benzene (3ac)

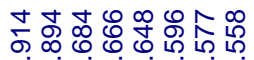

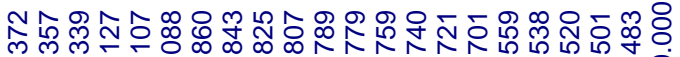

NiñNón

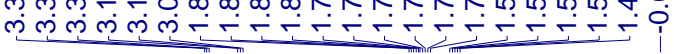

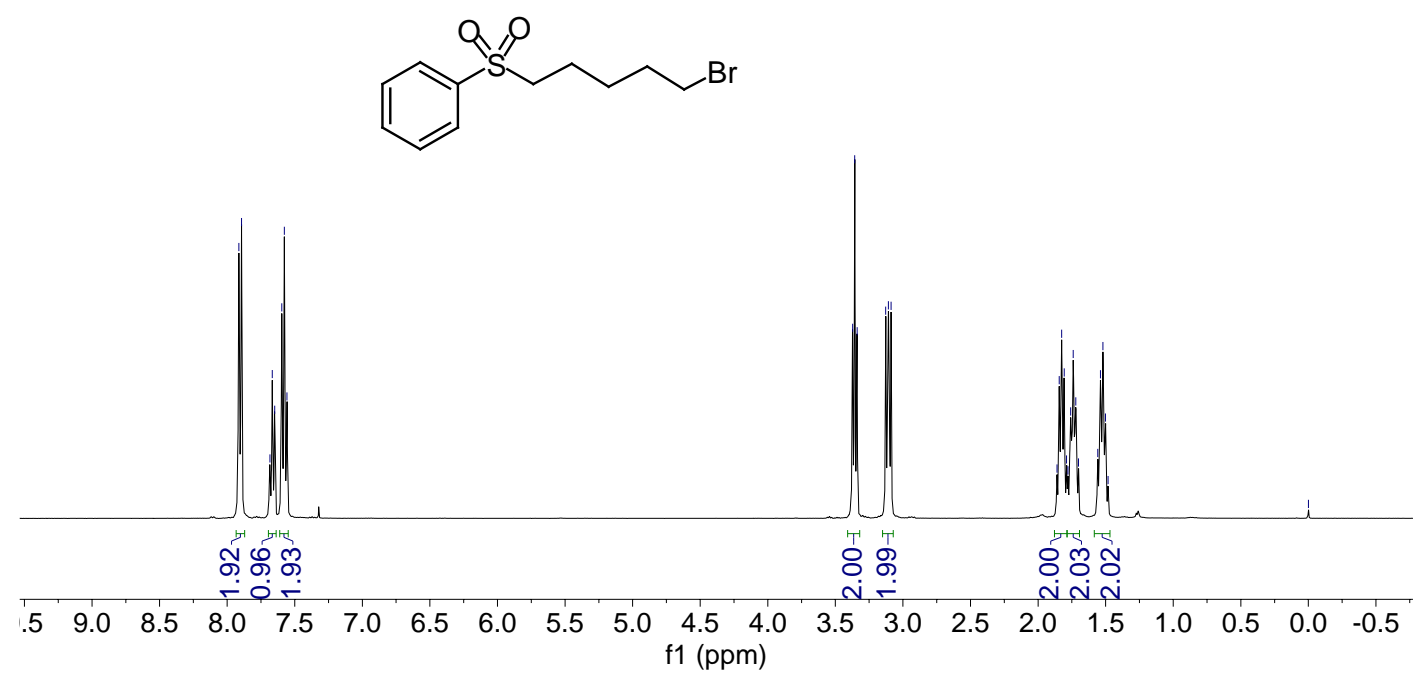

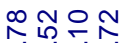

mं

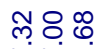

กิก゚

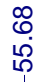

舟片母

广र

管

ले लू స<smiles>O=S(=O)(CCCCCBr)c1ccccc1</smiles>

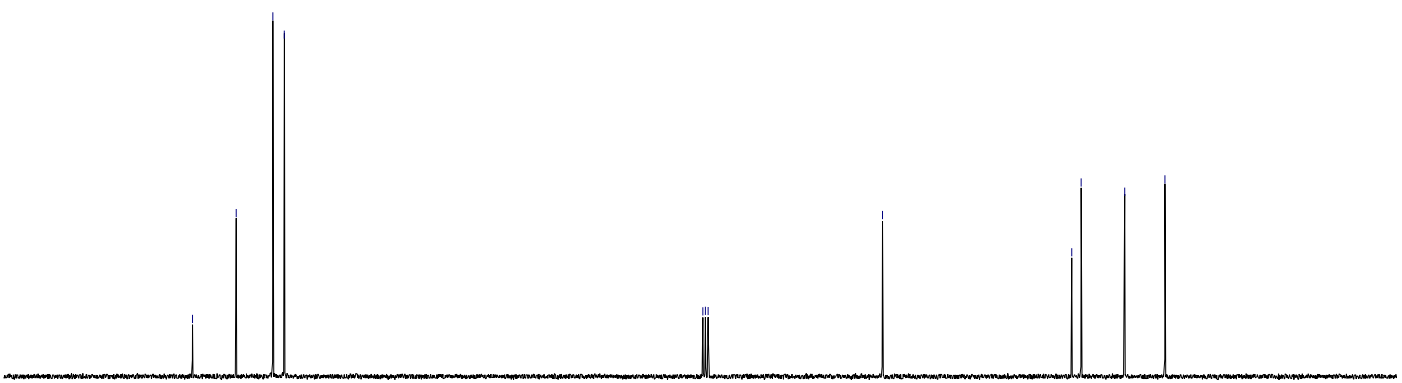

$60 \quad 150$

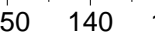

130120

O $110 \quad 100$

90

80
$\mathrm{f1}$ (ppm) 
$(8 R, 9 S, 13 S, 14 S)-13-M e t h y l-17-0 x 0-7,8,9,11,12,13,14,15,16,17-d e c a h y d r o-6 H$-cycl openta[a]phenanthren-3-yl 5-(phenylsulfonyl)pentanoate (3ad)

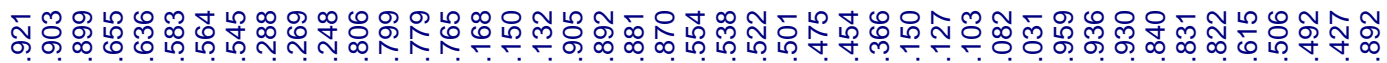

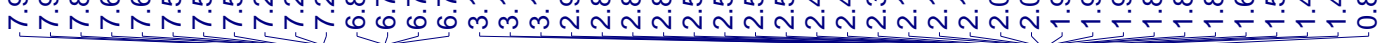<smiles>CCc1cc(OC(=O)CCCCS(=O)(=O)c2ccccc2)ccc1C1CCC2CC(=O)CCC21C</smiles>

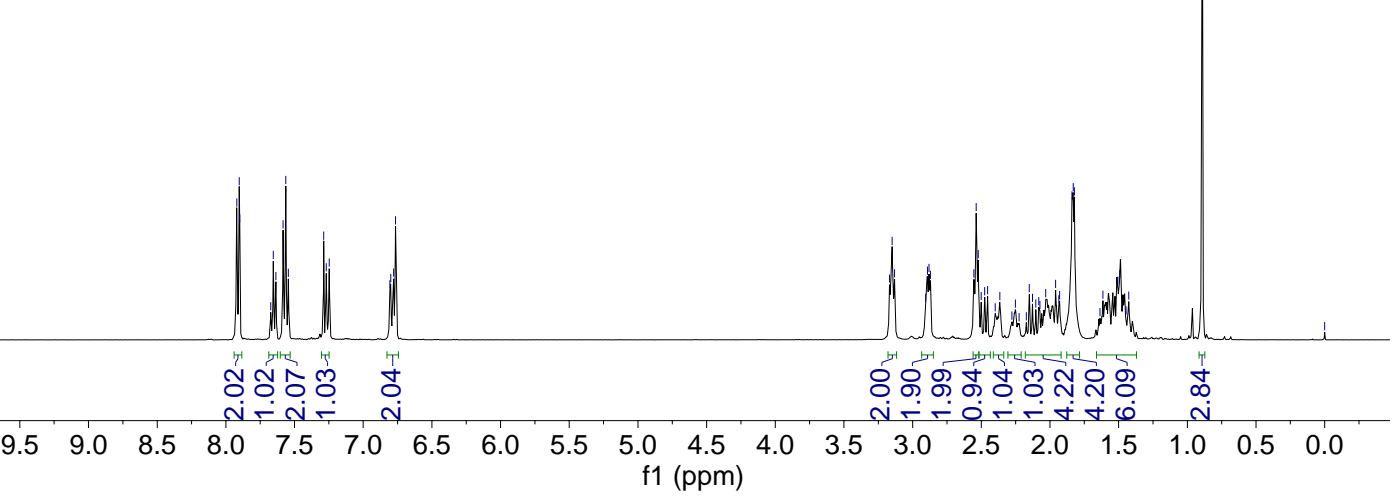

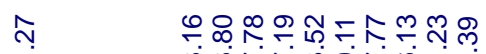

i $\quad$

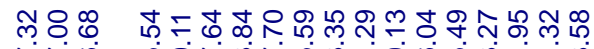

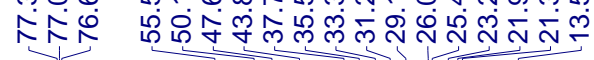

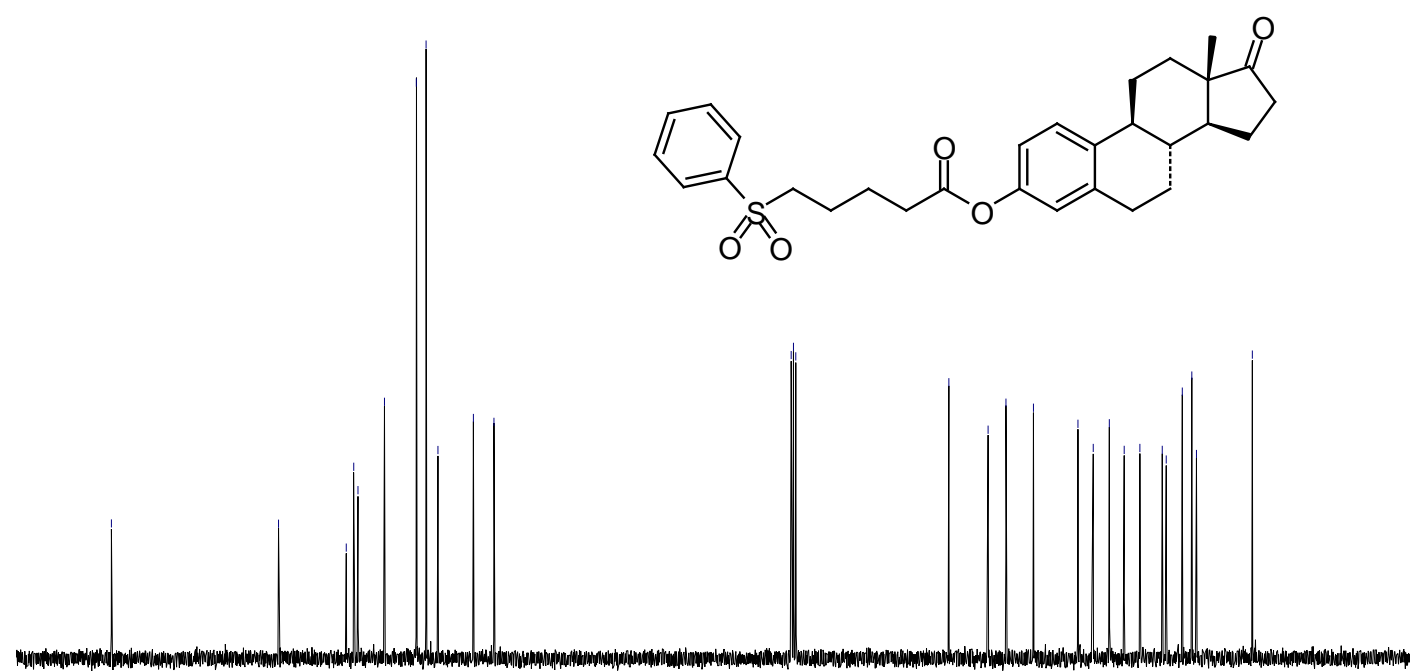

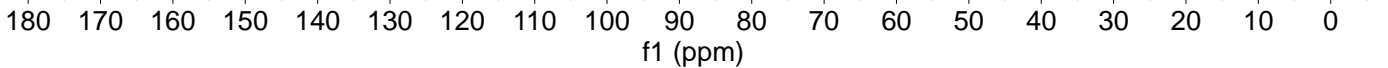


(Dodecylsulfonyl)benzene (3ae)

స్.

NNNNNNN

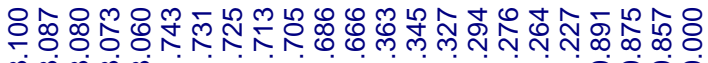

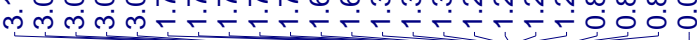
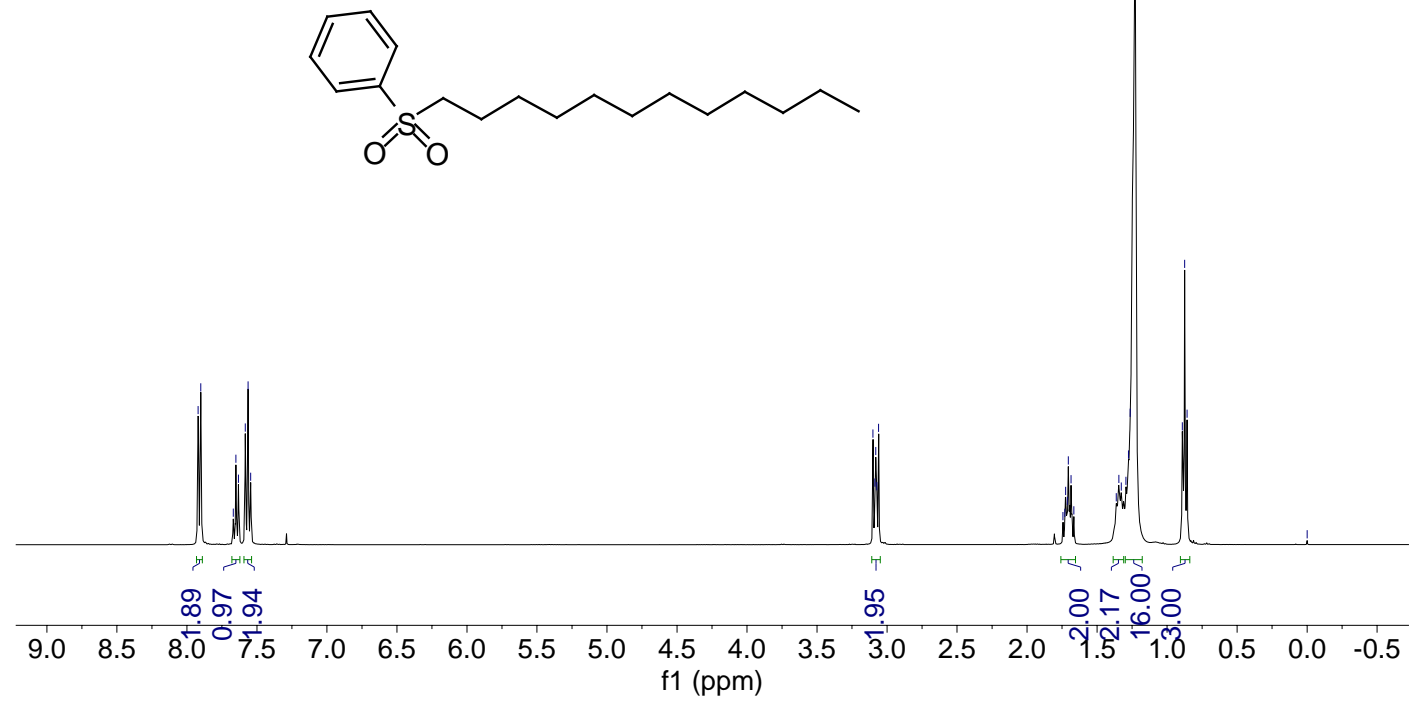

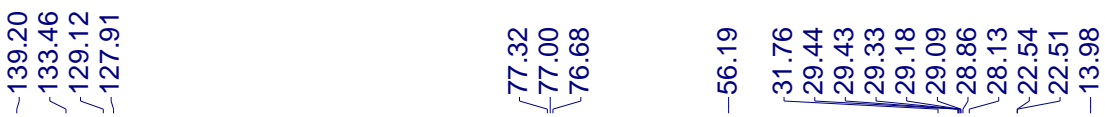

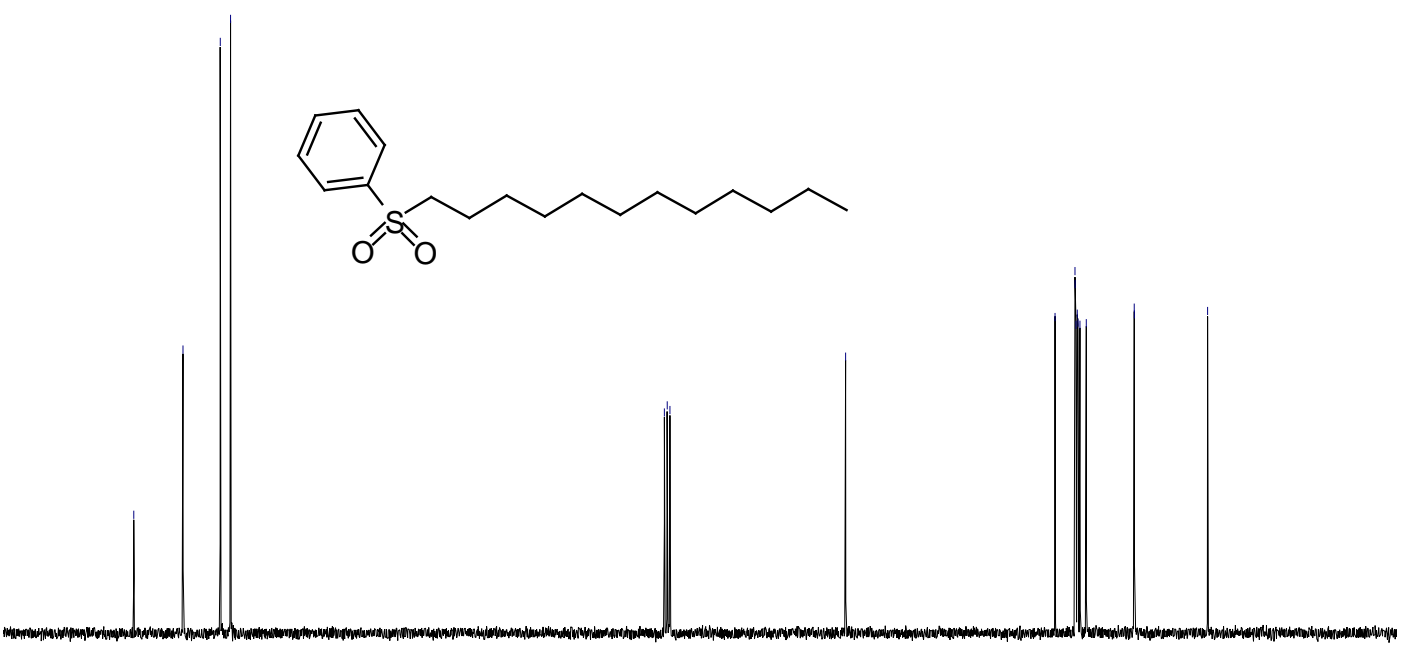

$\begin{array}{lllllllll}150 & 140 & 130 & 120 & 110 & 100 & 90 & 80 & 70\end{array}$ f1 (ppm) 


\section{((3-Phenylpropyl)sulfonyl)benzene (3af)}

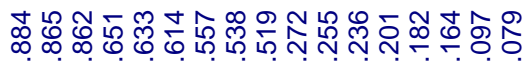

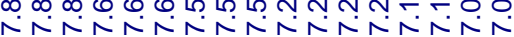

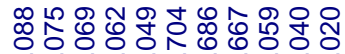

mं mं

$\stackrel{8}{8}$<smiles>O=S(=O)(CCCc1ccccc1)c1ccccc1</smiles>

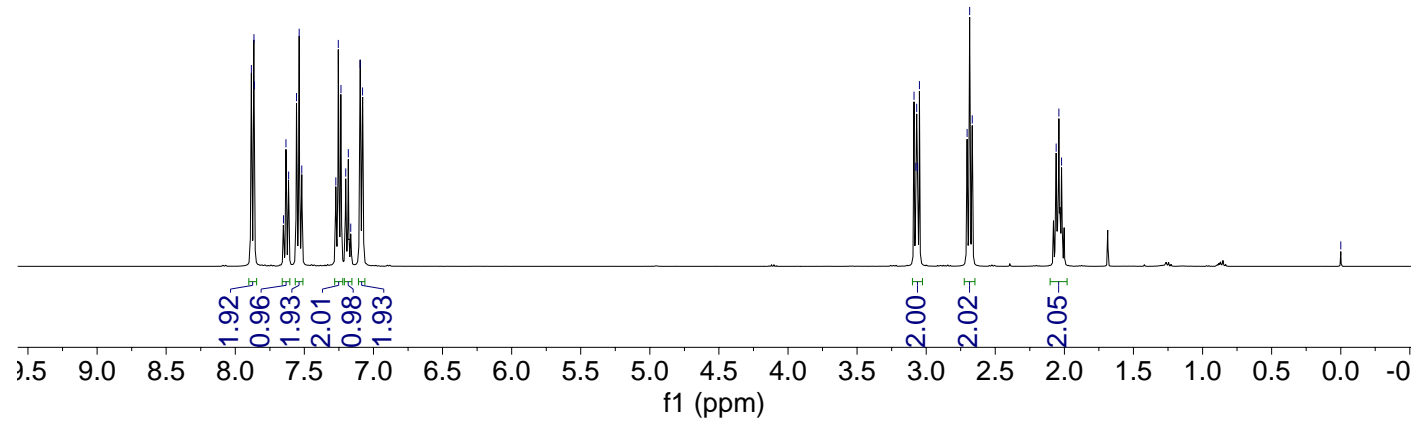

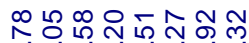

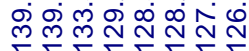

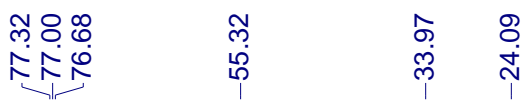

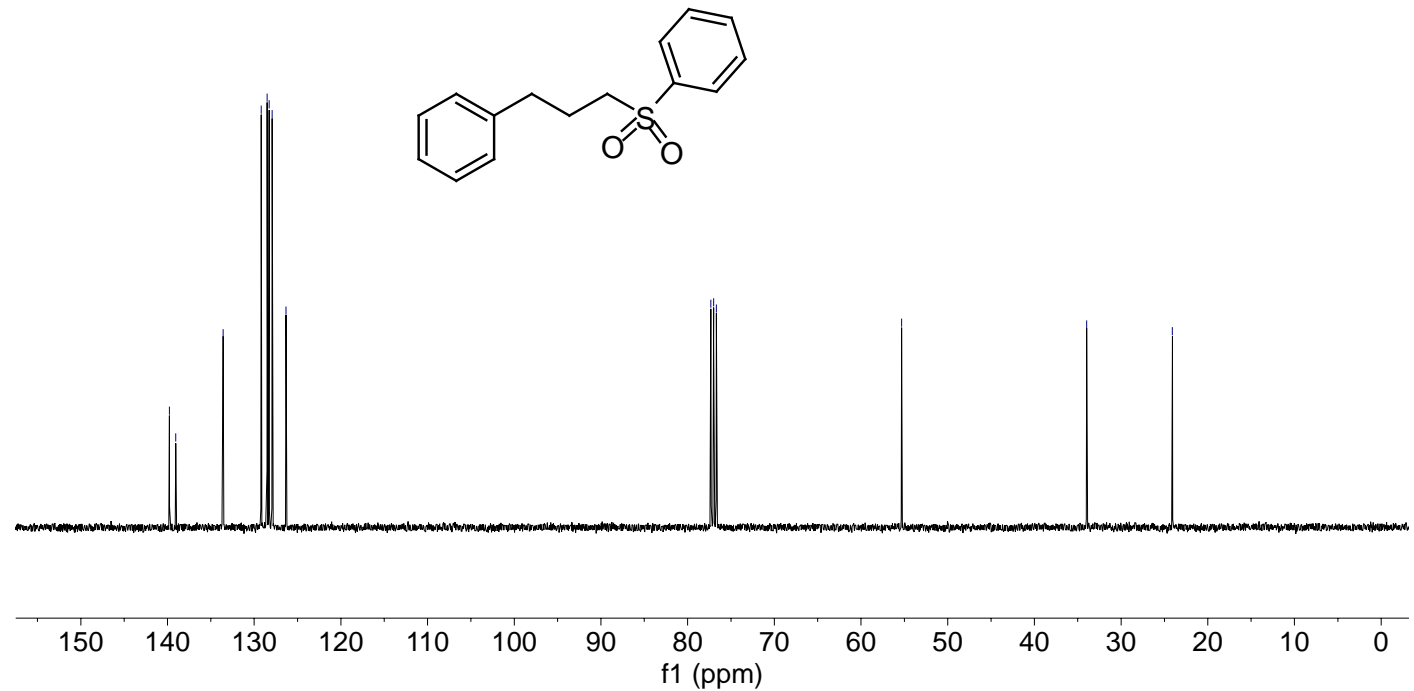




\section{((4-Phenylbutyl)sulfonyl)benzene (3ag)}

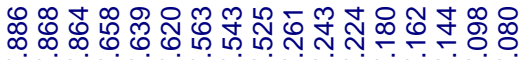

NNNNNNNNNNNNN

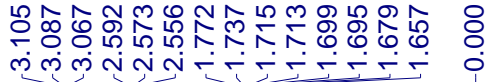

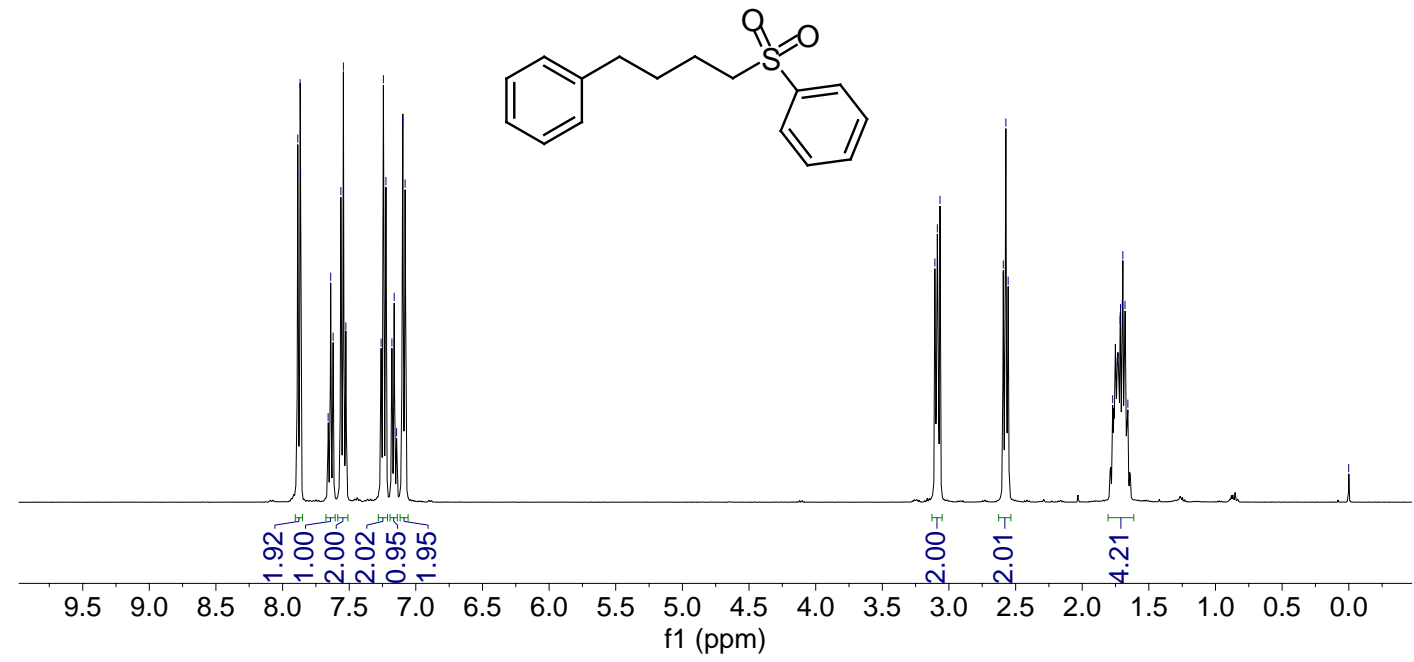

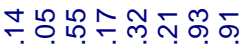

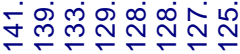

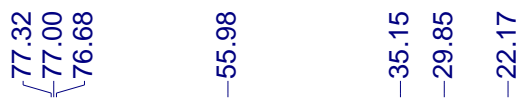

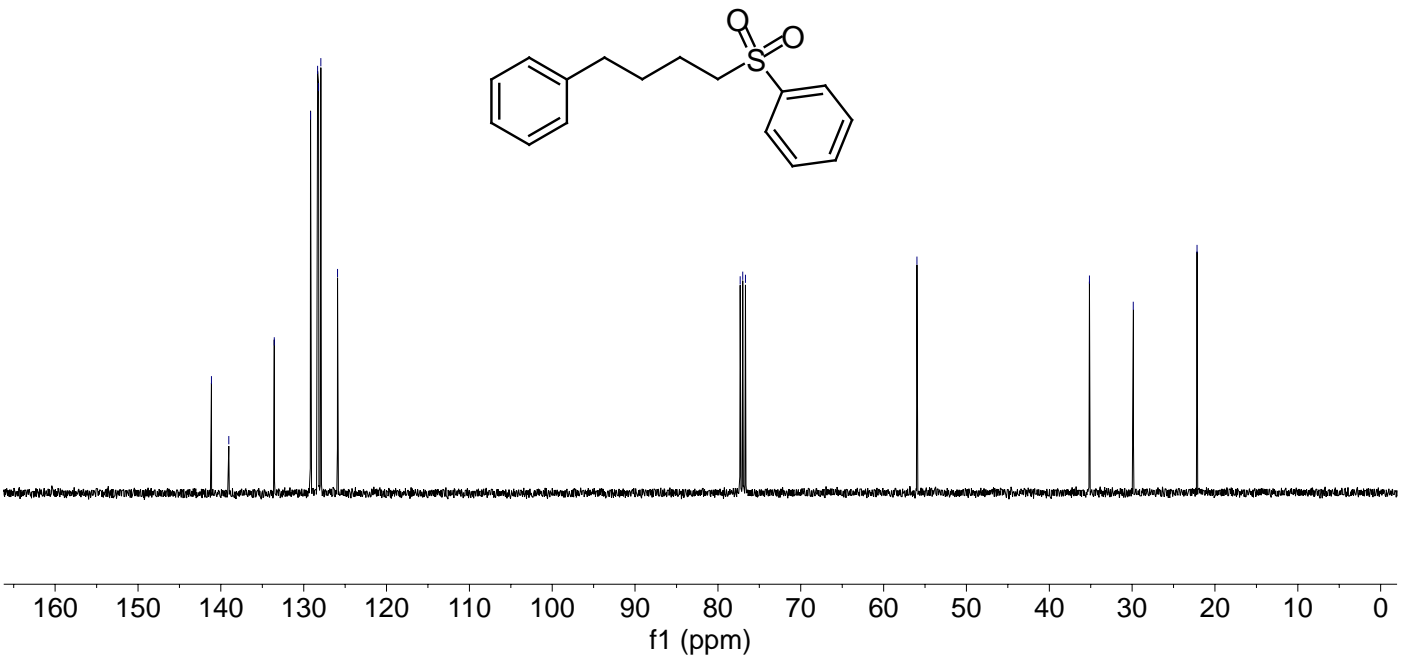


<smiles>O=S(=O)(CCC1CCCCC1)c1ccccc1</smiles>

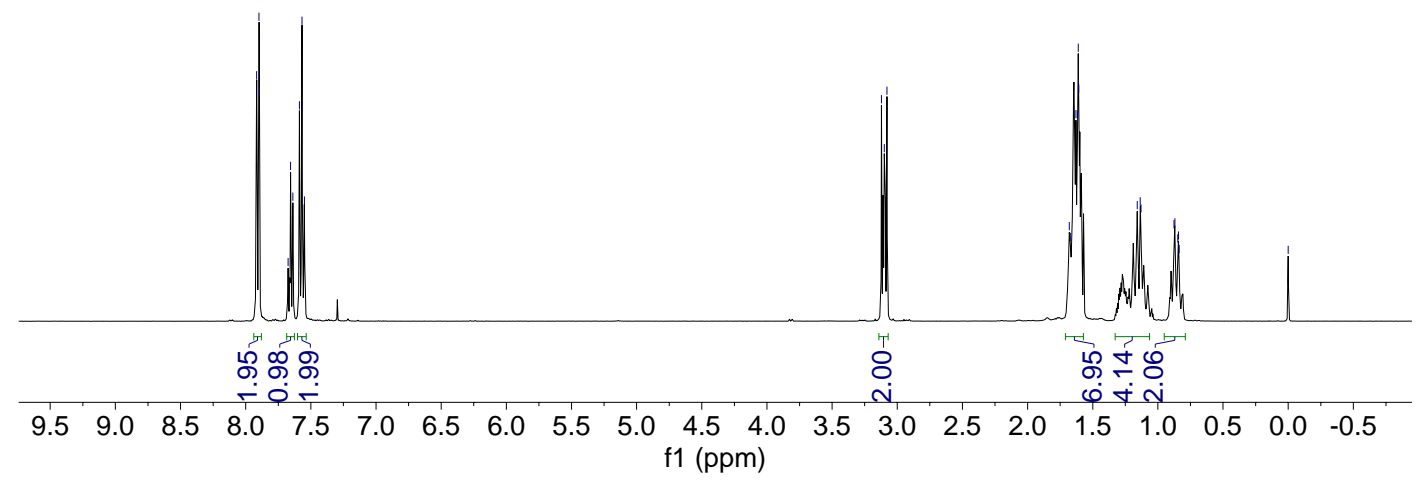

웅우는

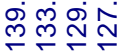

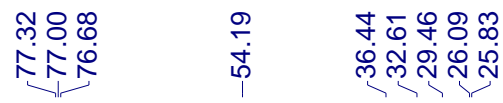
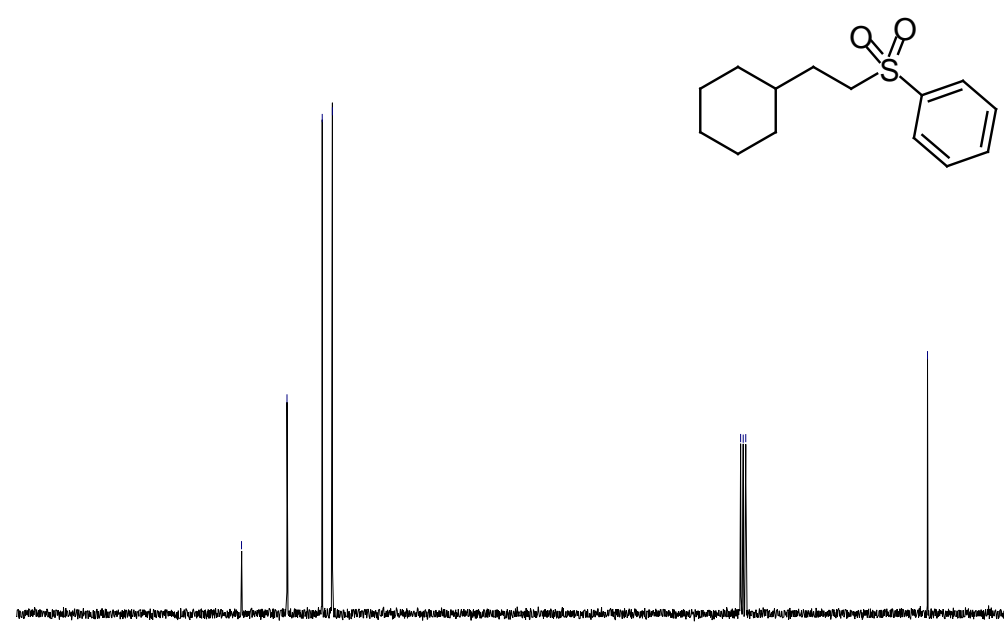

$160 \quad 150$

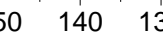

$\begin{array}{llll}30 & 120 & 110 & 100\end{array}$

$90 \begin{gathered}80 \\ f 1(p p m)\end{gathered}$ 


\section{$N$-Phenyl-5-(phenylsulfonyl)hexanamide (3ai-1)}

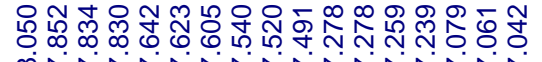

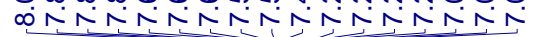

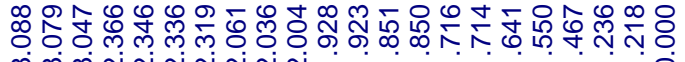

ल ल लंN

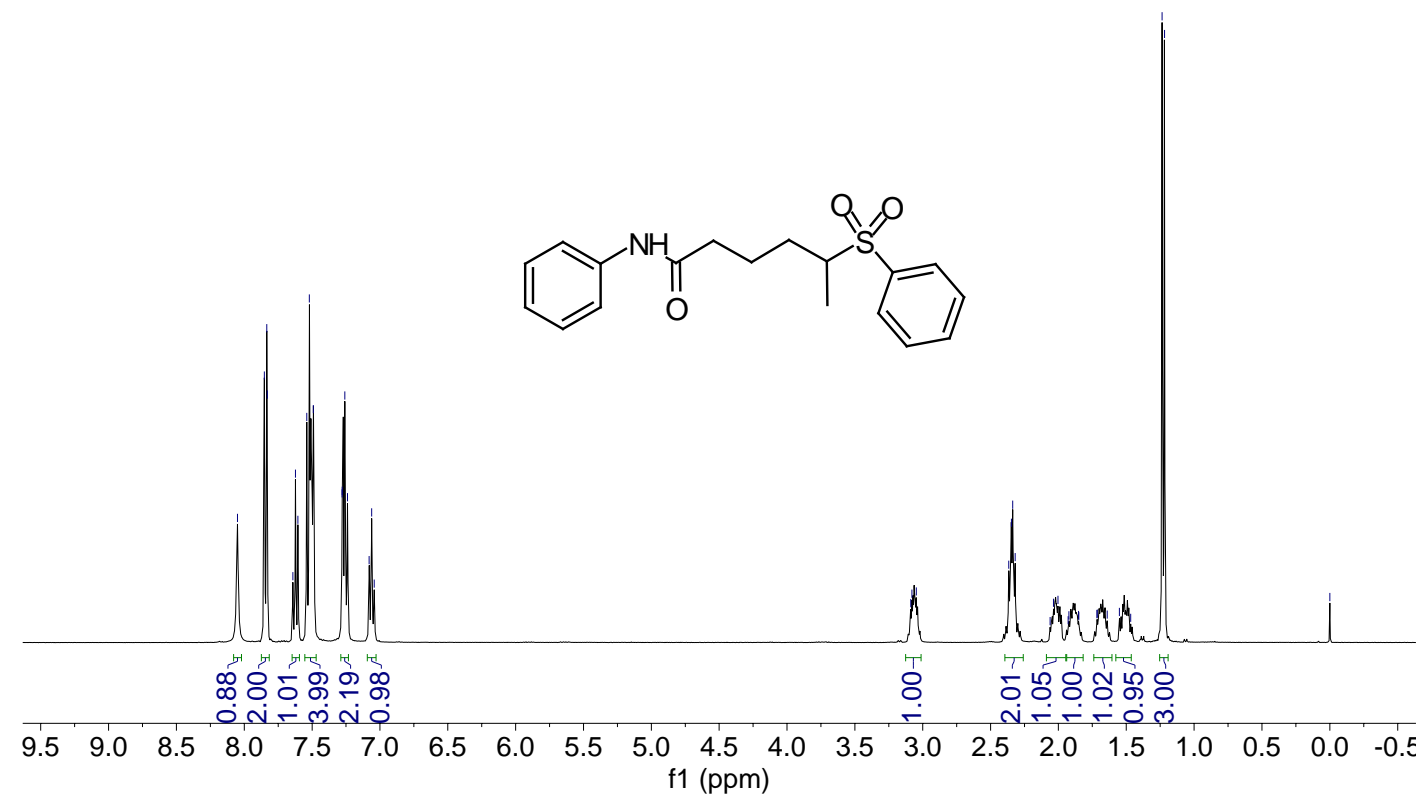

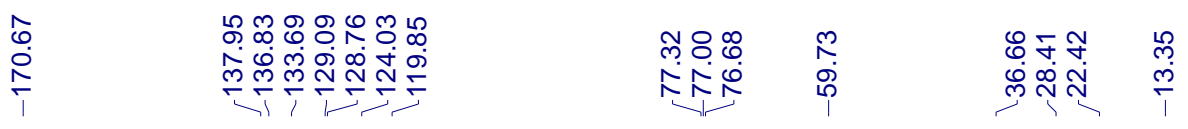

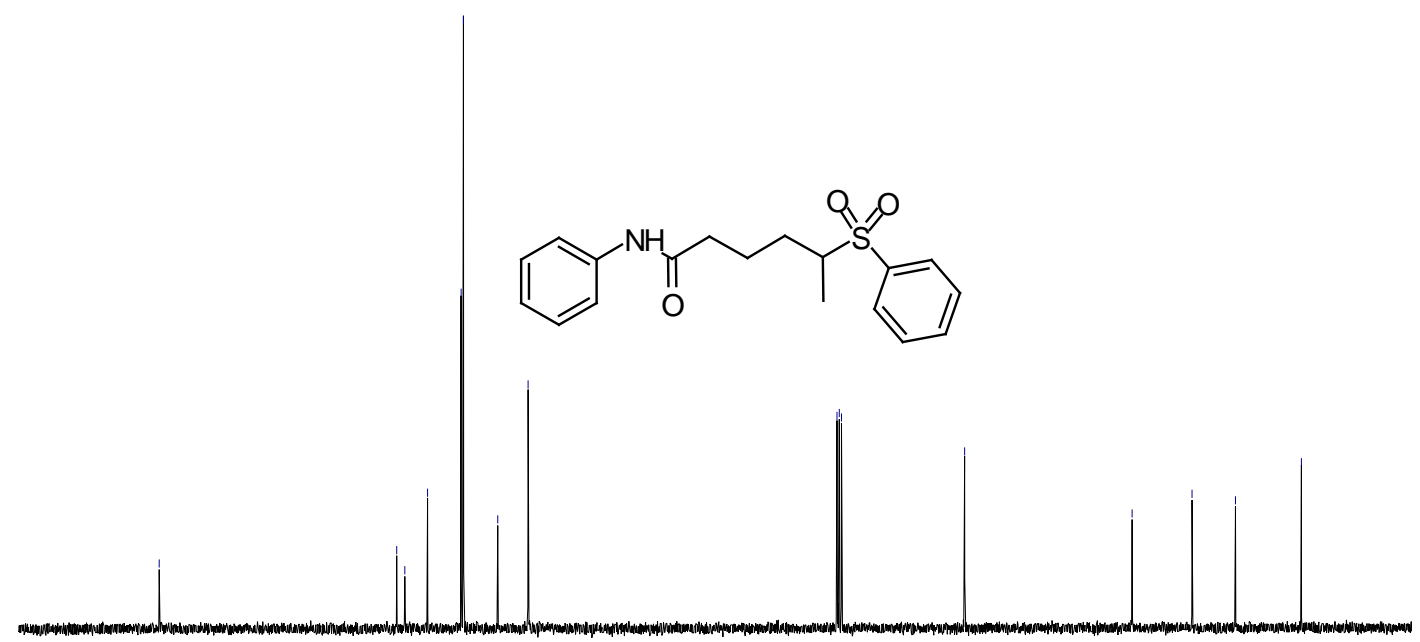

\begin{tabular}{lllllllllllllllllllll}
\hline 0 & 180 & 170 & 160 & 150 & 140 & 130 & 120 & 110 & $\begin{array}{r}100 \\
\mathrm{f} 1(\mathrm{ppm})\end{array}$ & 80 & 70 & 60 & 50 & 40 & 30 & 20 & 10 & 0
\end{tabular} 


\section{$N$-Phenyl-4-(phenylsulfonyl)hexanamide (3ai-2)}

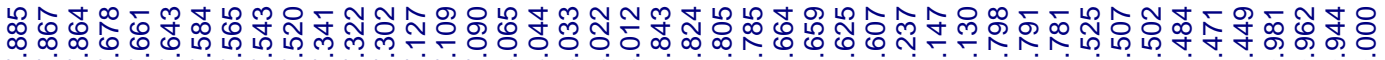

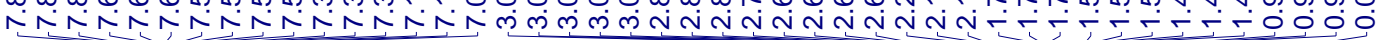

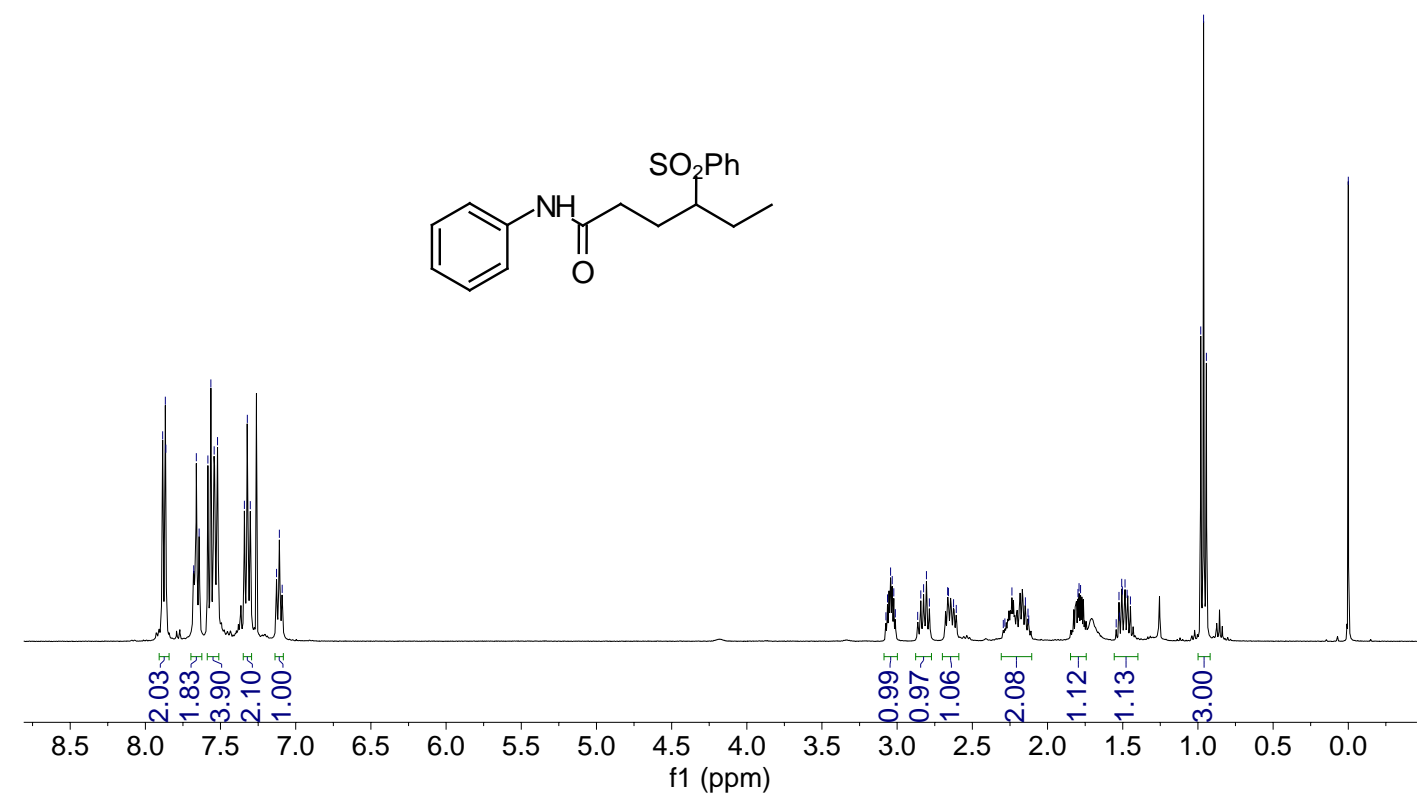

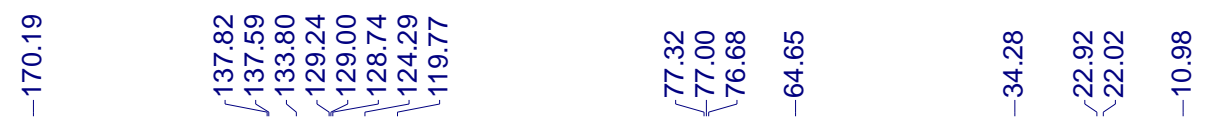<smiles>CCC(CCC(=O)Nc1ccccc1)c1ccccc1</smiles> 


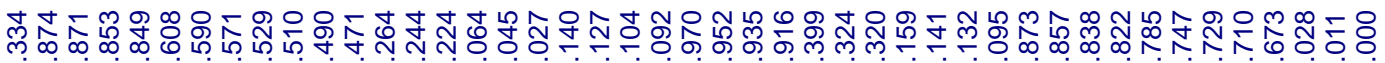

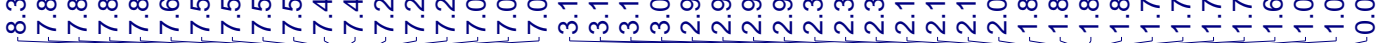

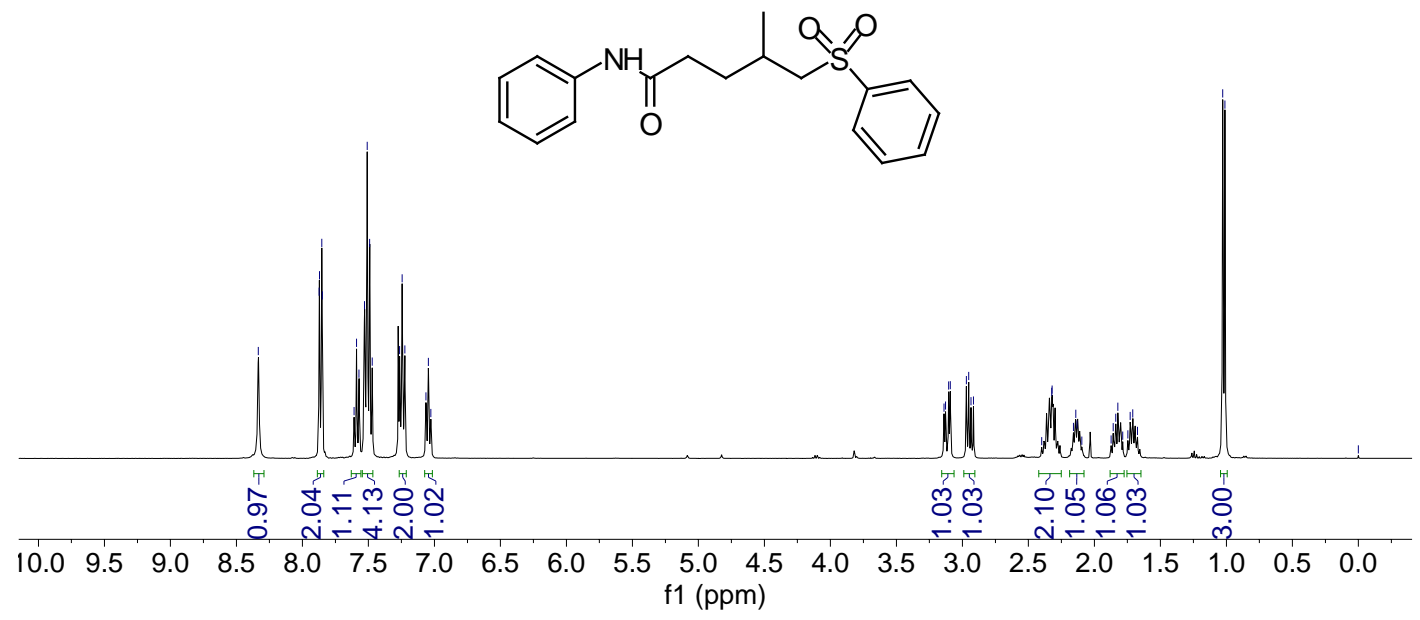

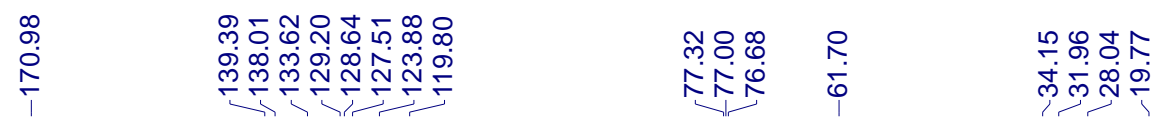

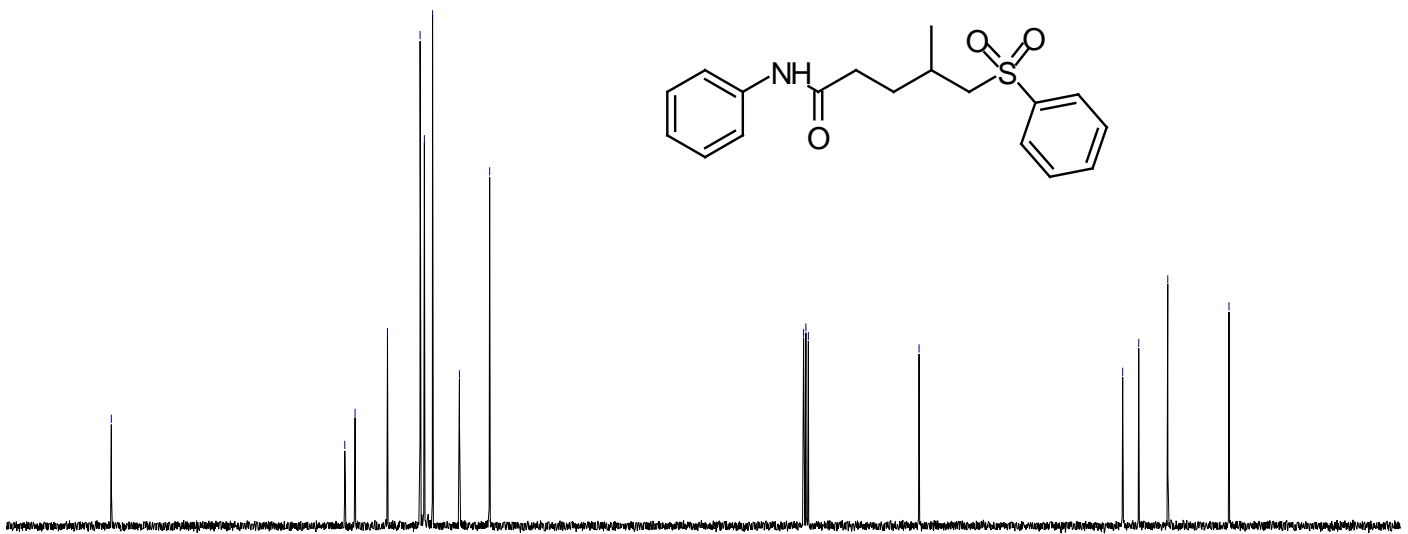

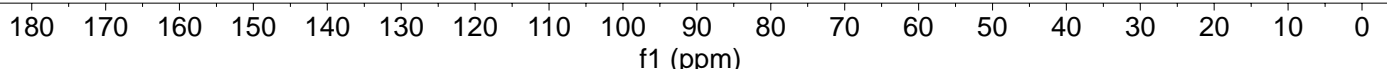


((2-Ethylbutyl)sulfonyl)benzene (3ak)

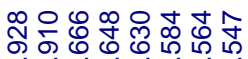

Nin

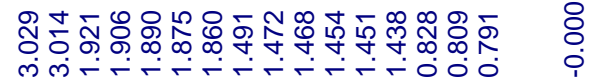<smiles>CCC(CC)CS(=O)(=O)c1ccccc1</smiles>

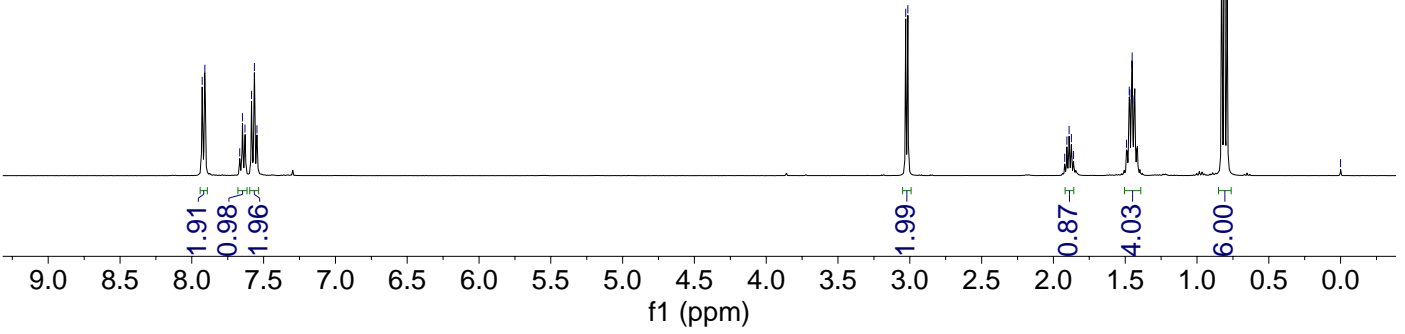

우요둥

ปัต ํํำ

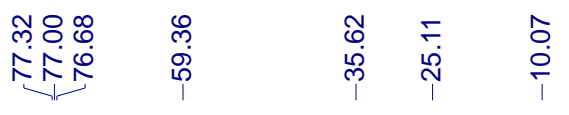

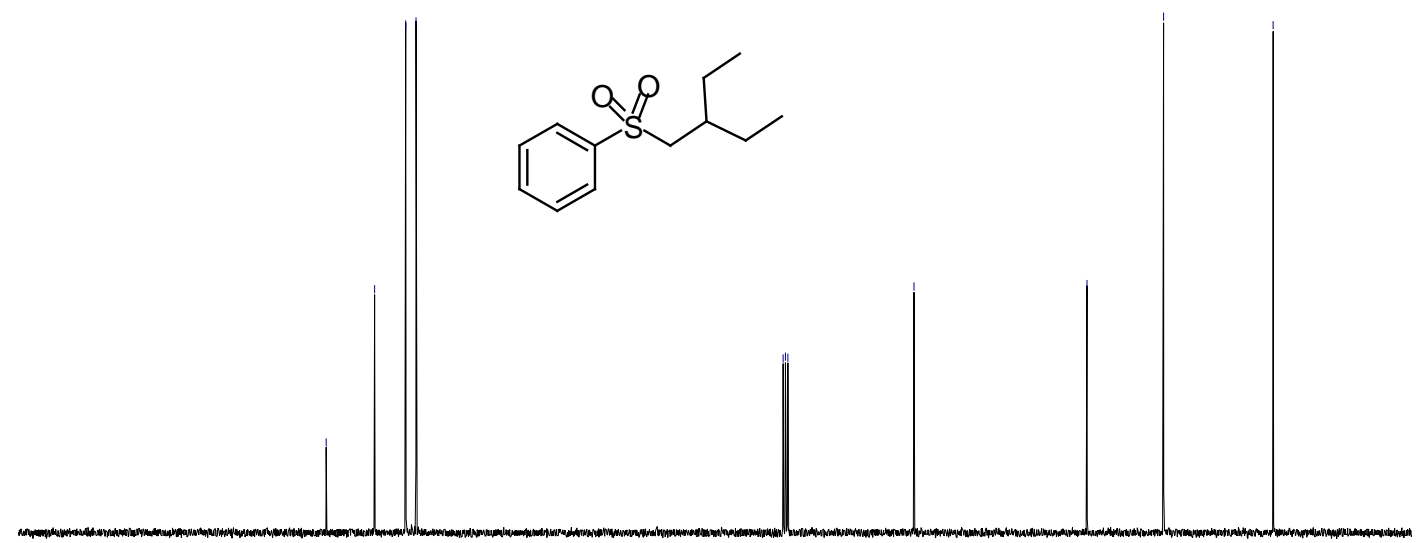

$\begin{array}{lllllllllllllllllllll}180 & 170 & 160 & 150 & 140 & 130 & 120 & 110 & 100 & 90 & 80 & 70 & 60 & 50 & 40 & 30 & 20 & 10 & 0\end{array}$ 
((2,3-Dimethylbutan-2-yl)sulfonyl)benzene (3al)

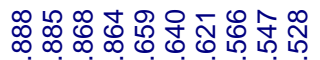

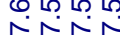

㐫

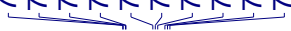

i

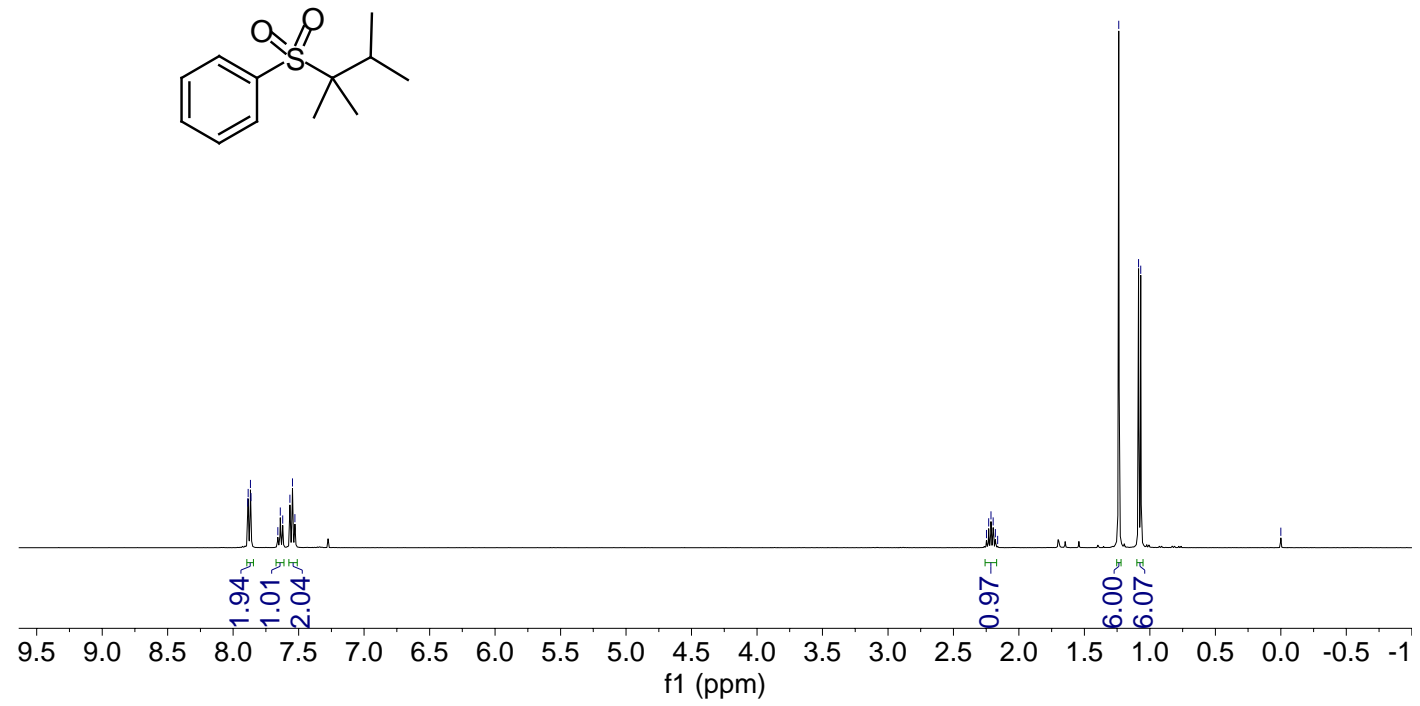

พฺฺุ

i্

ల్లిం:

사요용

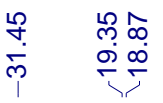<smiles>CC(C)C(C)(C)S(=O)(=O)c1ccccc1</smiles>

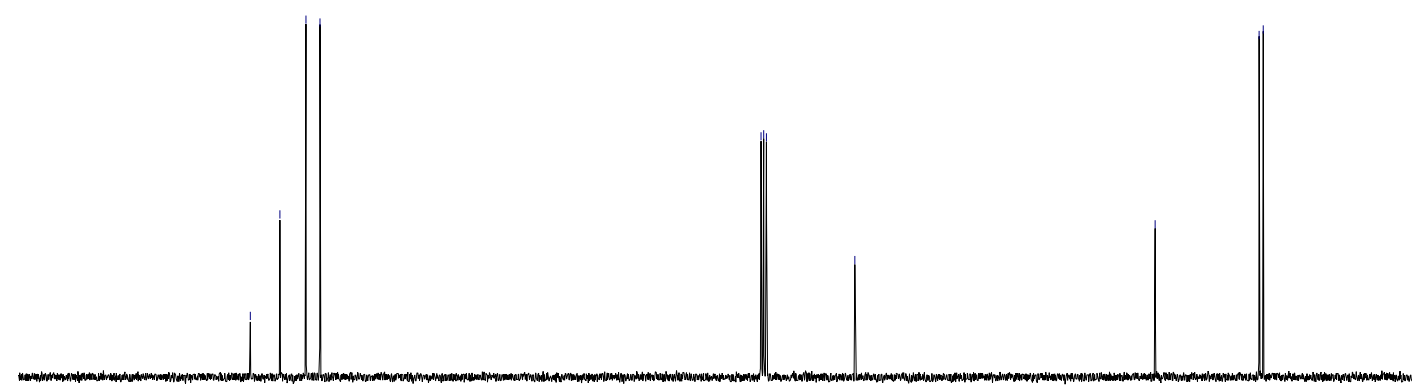

160

$\begin{array}{llll}150 & 140 & 130 & 1\end{array}$

20110

$90 \quad 80$

$70 \quad 60$

$\begin{array}{lllll}50 & 40 & 30 & 20 & 10\end{array}$ 
Methyl 2-(phenylsulfonyl)cyclopentane-1-carboxylate (3am)

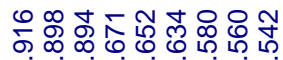

ríñNiñ

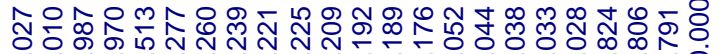

ナ

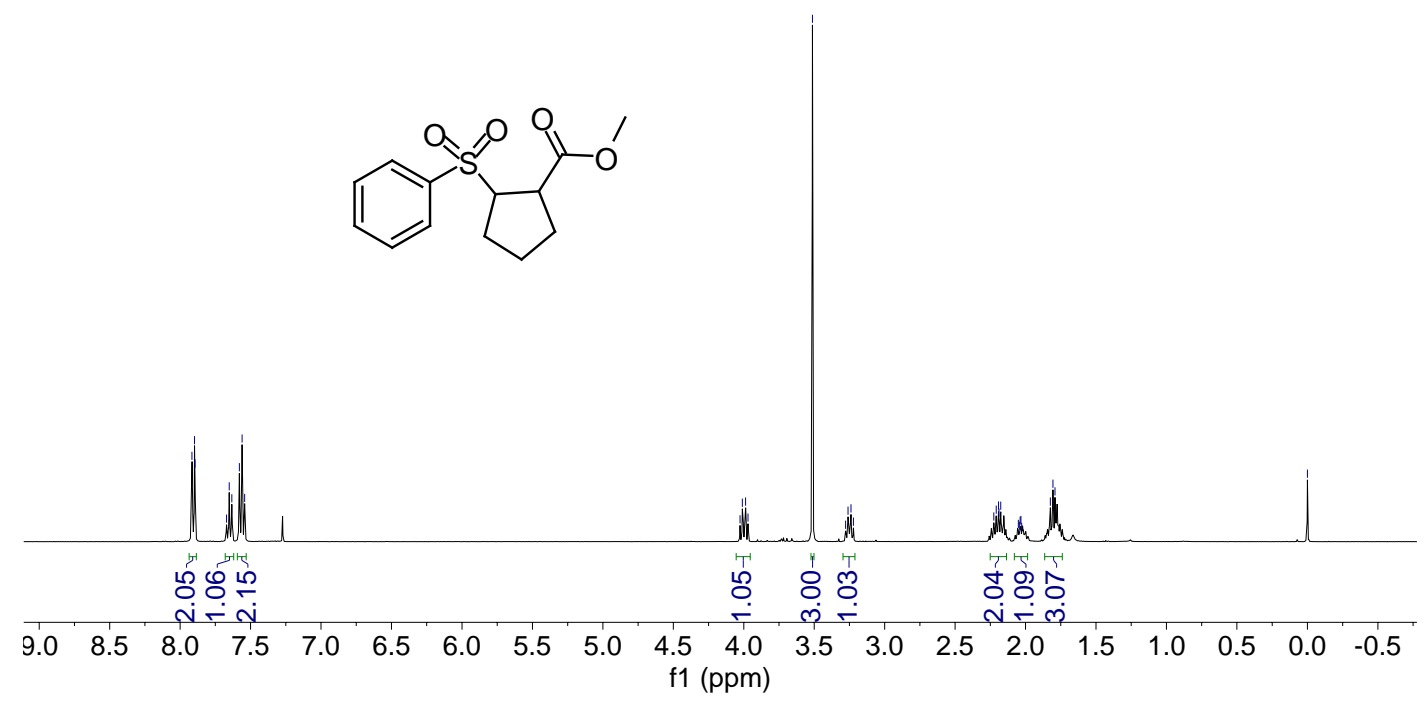

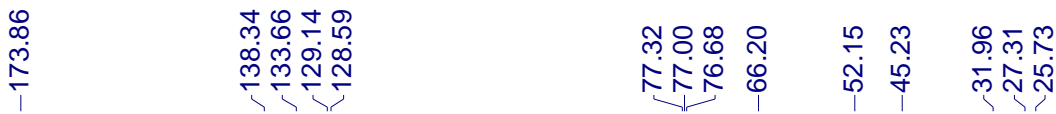

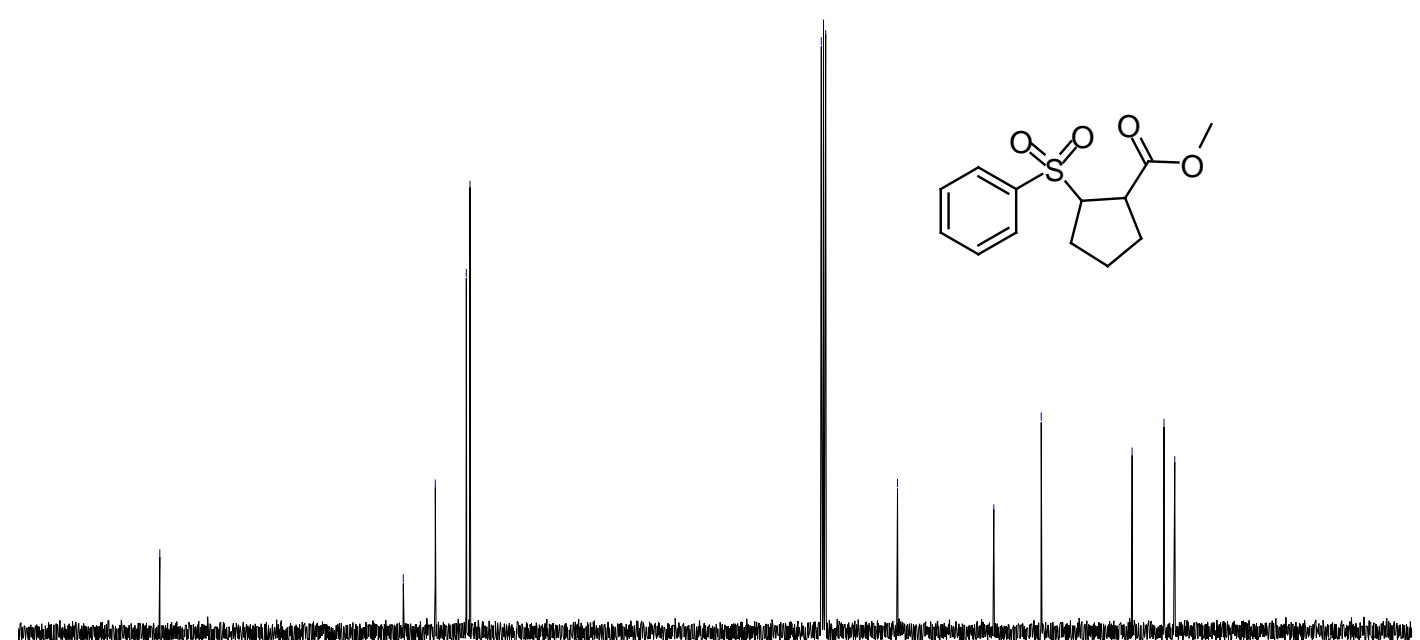

$\begin{array}{llllllllllllllllllll}190 & 180 & 170 & 160 & 150 & 140 & 130 & 120 & 110 & \begin{array}{c}100 \\ \mathrm{f} 1(\mathrm{ppm})\end{array} & 80 & 70 & 60 & 50 & 40 & 30 & 20 & 10 & 0\end{array}$ 


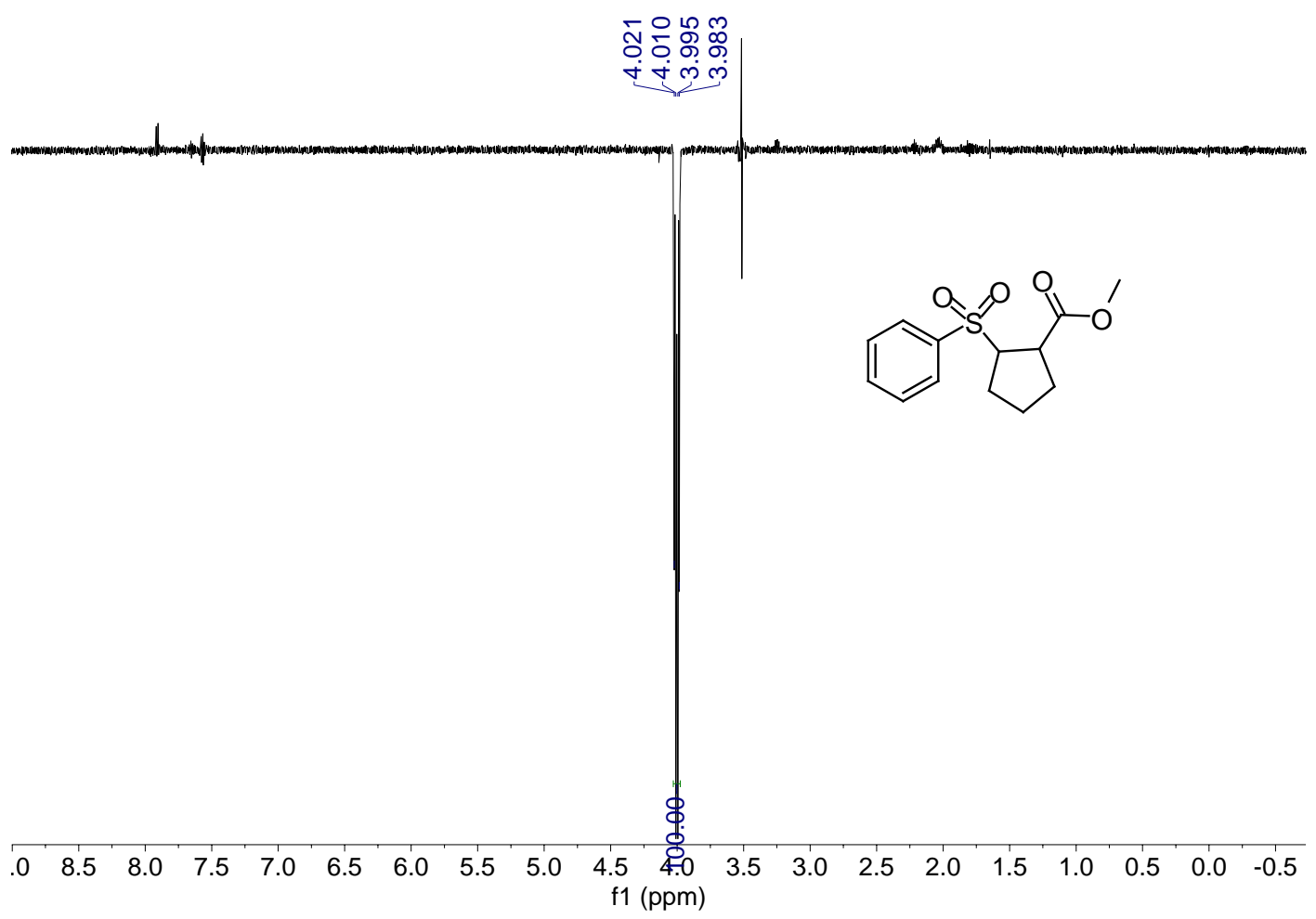

S74 


\section{$N$,2-Dimethyl-N-phenyl-3-(phenylsulfonyl)propanamide (3an)}
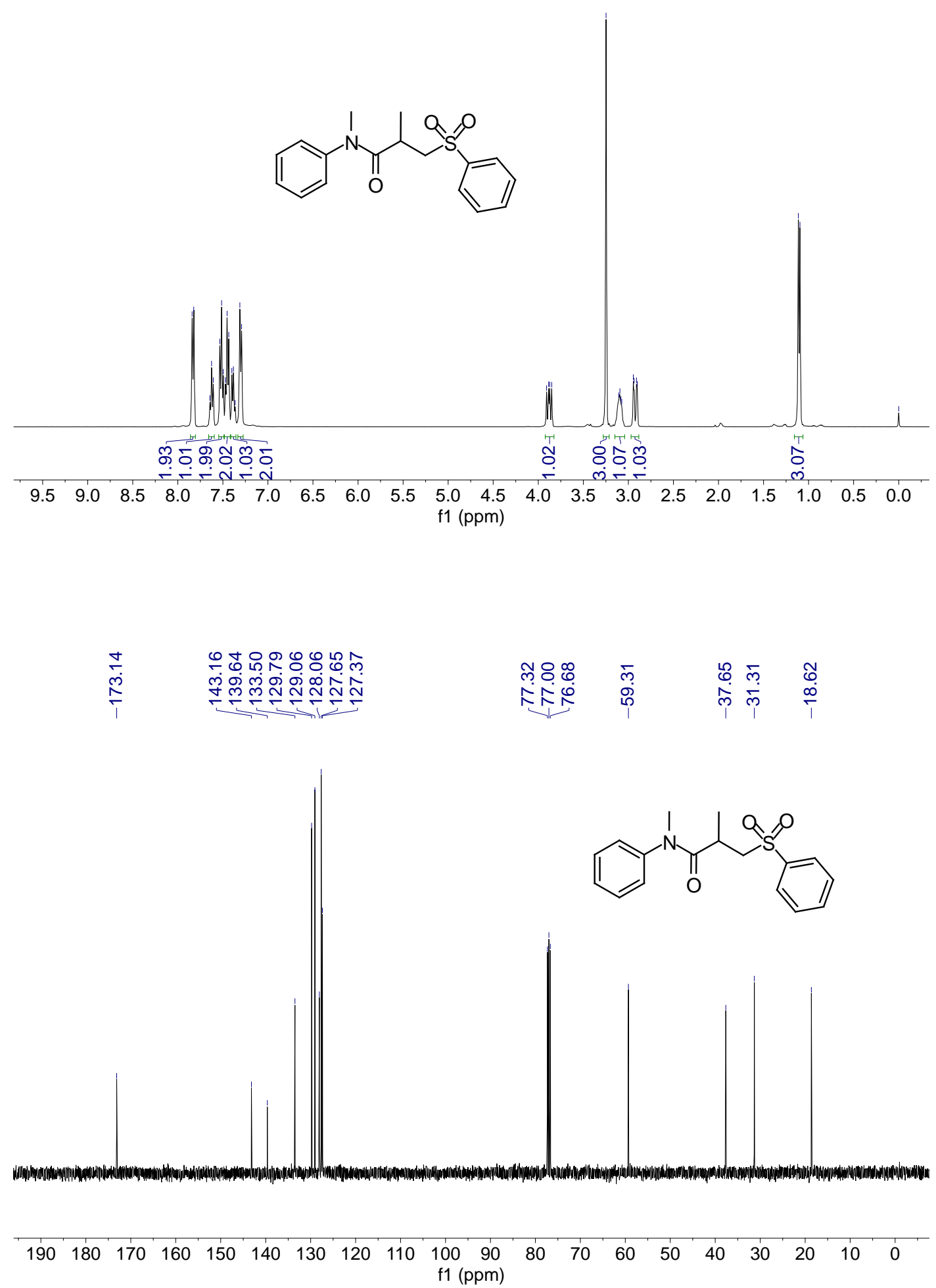
2-(Phenylsulfonyl)ethyl acetate (3ap)

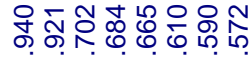

NNNNNNN

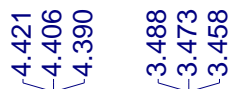

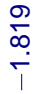

8
8
0
1

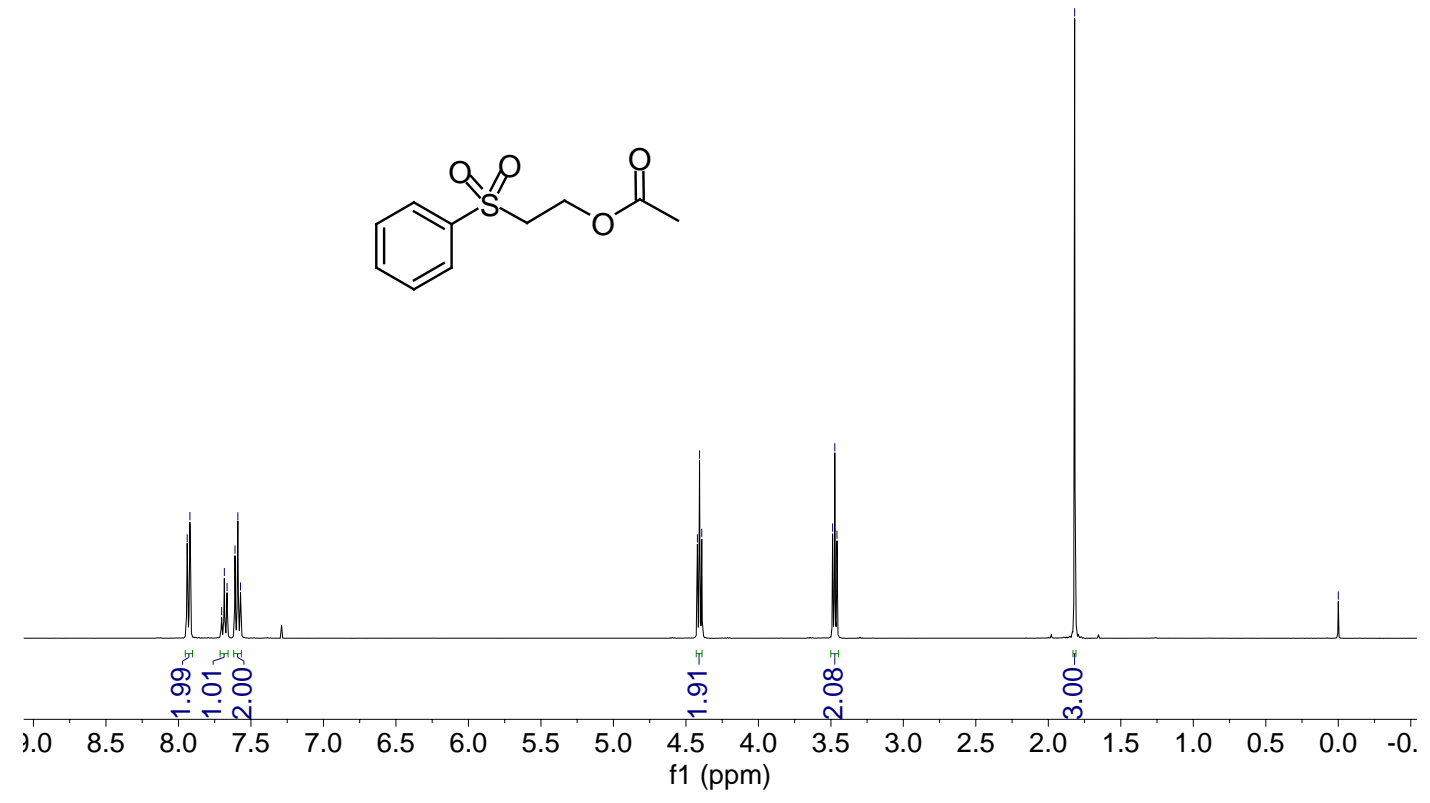

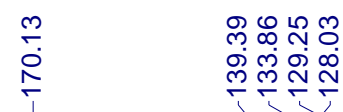

లి

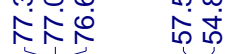

胥

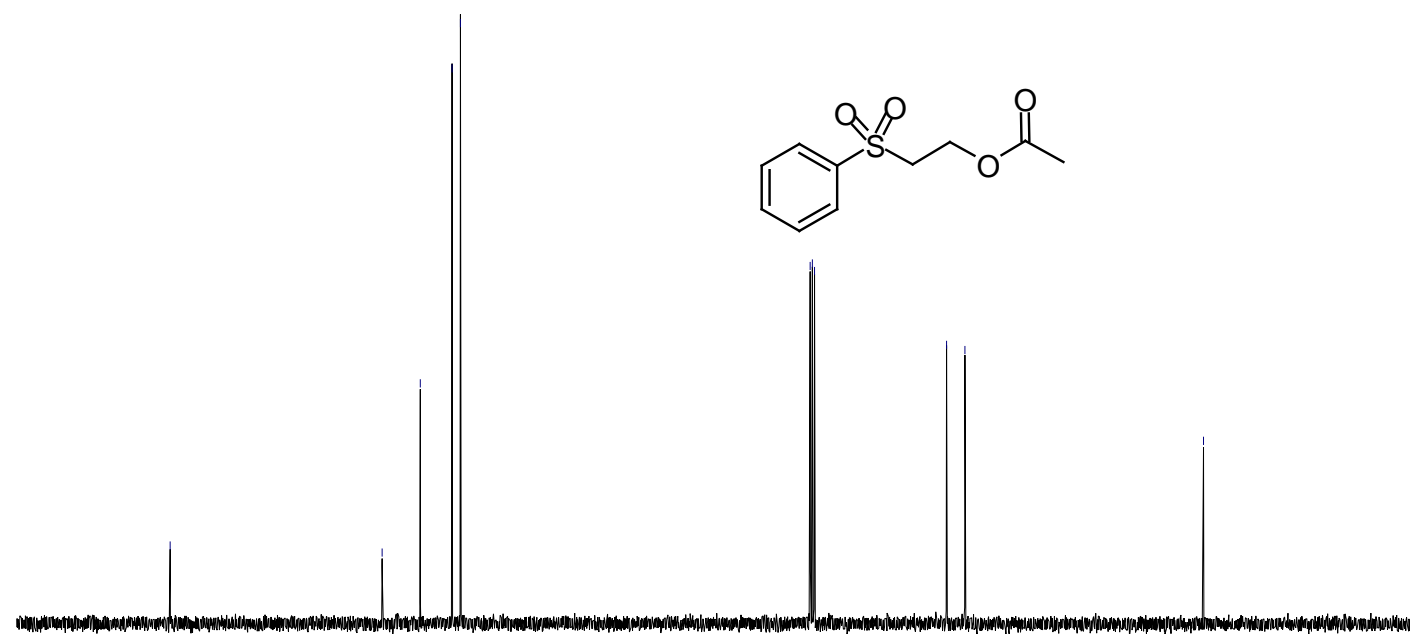

$\begin{array}{llllllllllllllllllllllll}190 & 180 & 170 & 160 & 150 & 140 & 130 & 120 & 110 & 100 & 90 & 80 & 70 & 60 & 50 & 40 & 30 & 20 & 10 & 0\end{array}$ 


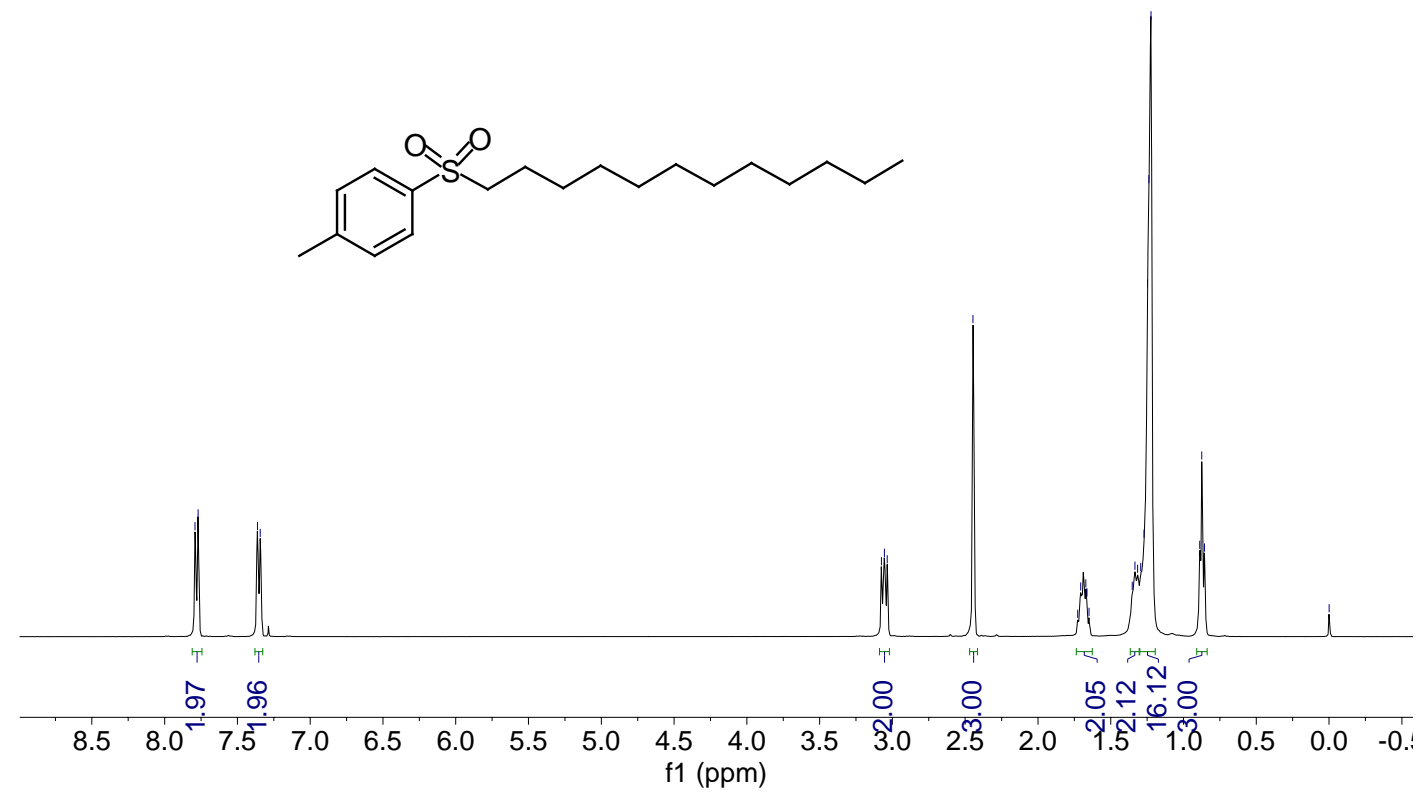

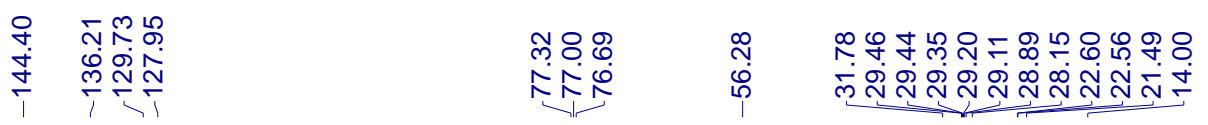

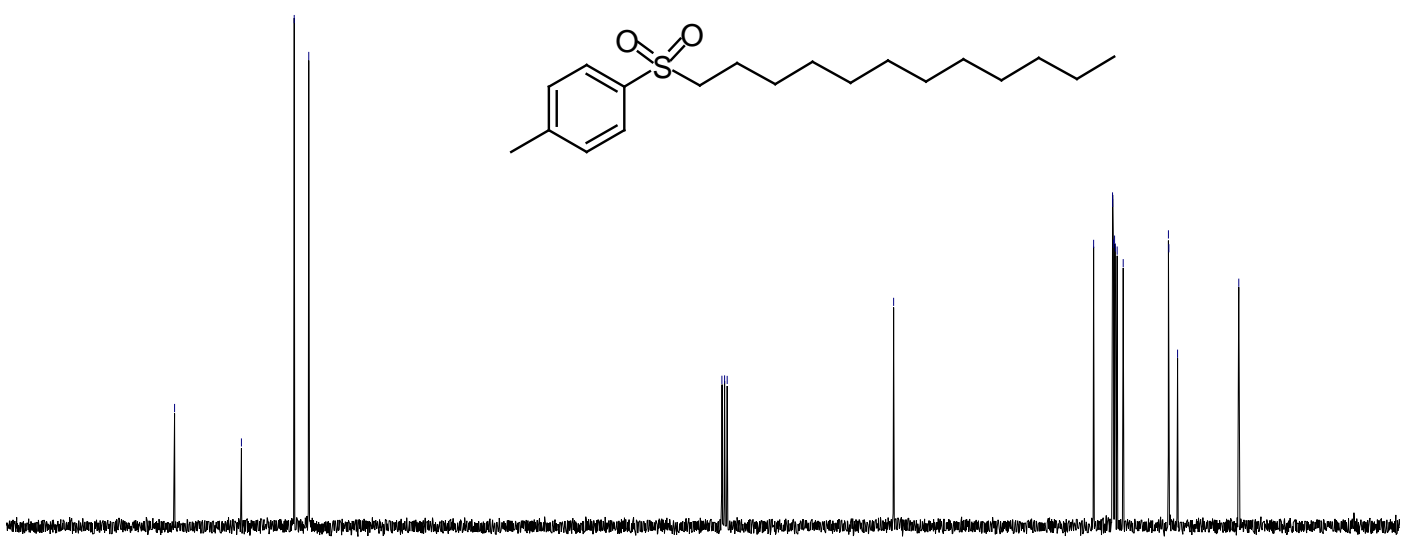

$\begin{array}{lllllllllllllllll}160 & 150 & 140 & 130 & 120 & 110 & 100 & 90 & \begin{array}{c}80 \\ \mathrm{f} 1(\mathrm{ppm})\end{array} & 70 & 60 & 50 & 40 & 30 & 20 & 10 & 0\end{array}$ 
1-(Dodecylsulfonyl)-4-methoxybenzene (4b)

חָষ

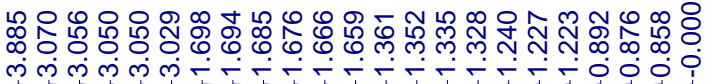

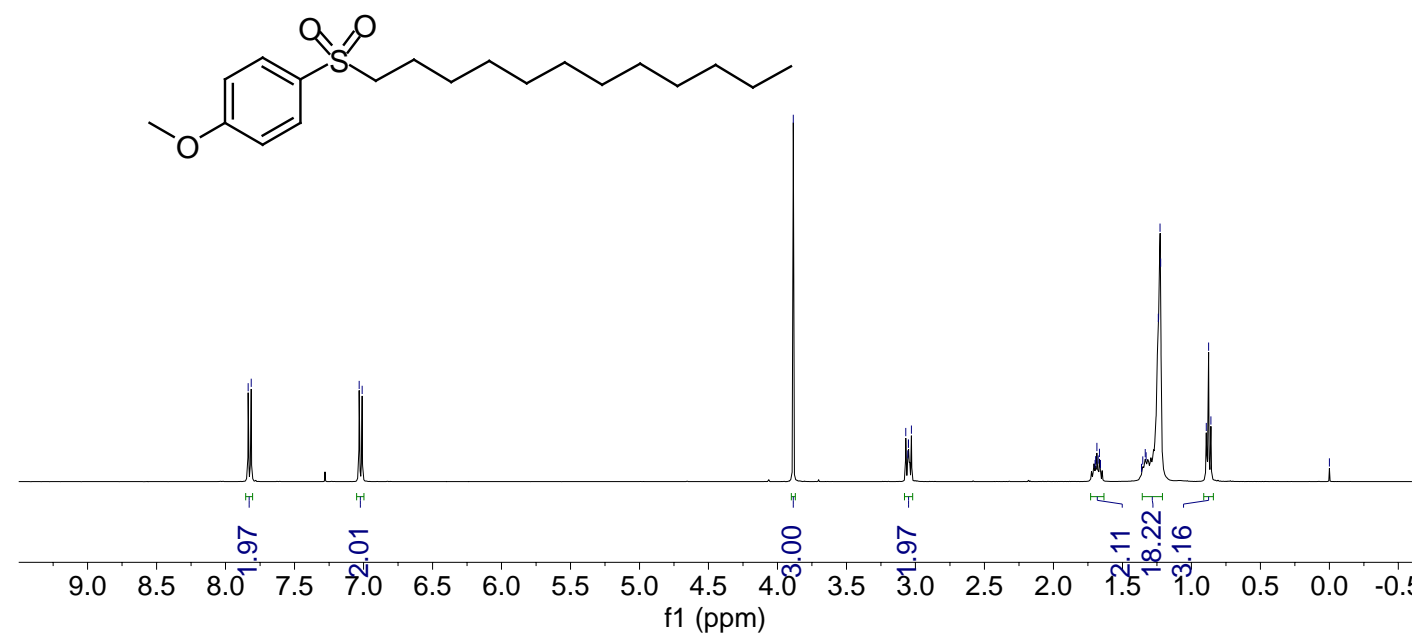

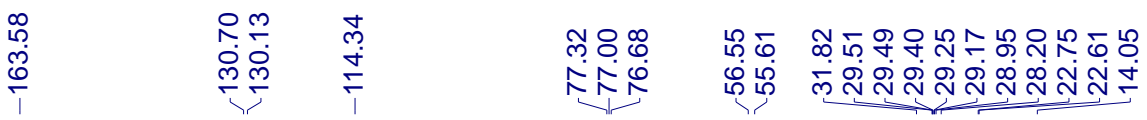<smiles>CCCCCCCCCCCCS(=O)(=O)c1ccc(OC)cc1</smiles>

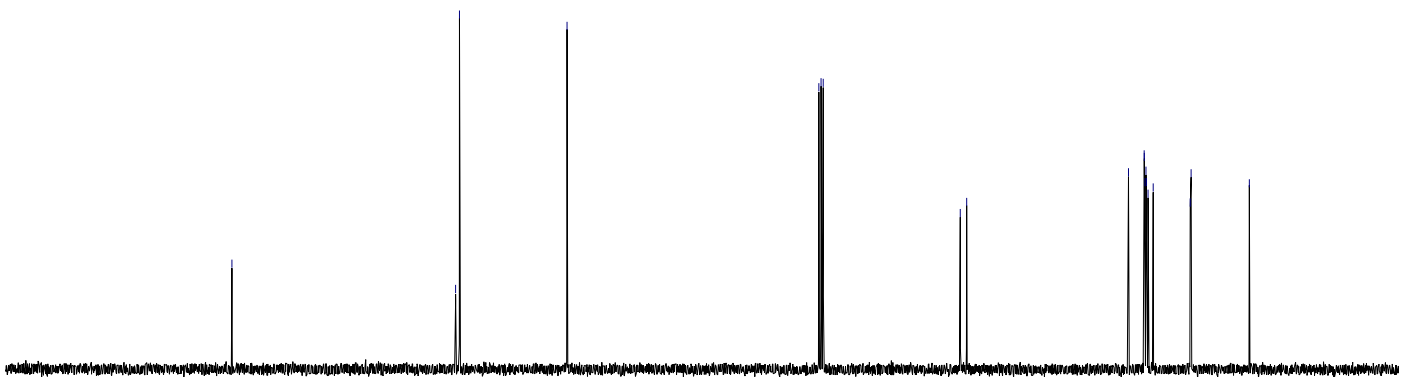

$\begin{array}{llllllllllllllllllll}190 & 180 & 170 & 160 & 150 & 140 & 130 & 120 & 110 & 100 & 90 & 80 & 70 & 60 & 50 & 40 & 30 & 20 & 10 & 0\end{array}$ f1 (ppm) 
1-(Dodecylsulfonyl)-4-fluorobenzene (4c)

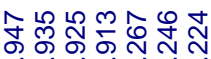

riñNiñ

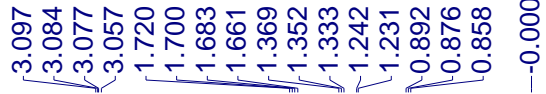
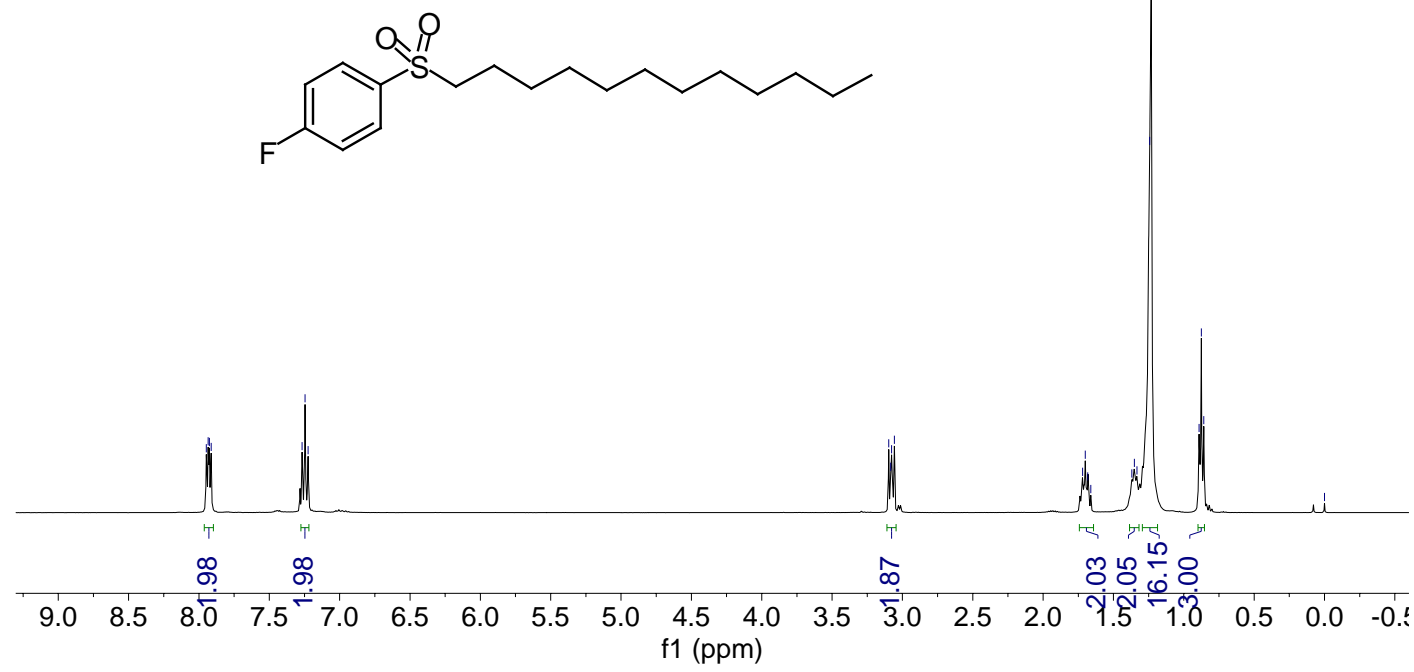

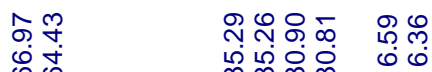

ம்

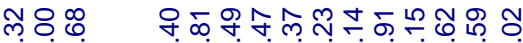

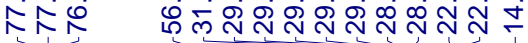<smiles>CCCCCCCCCCCCCS(=O)(=O)c1ccc(F)cc1</smiles>

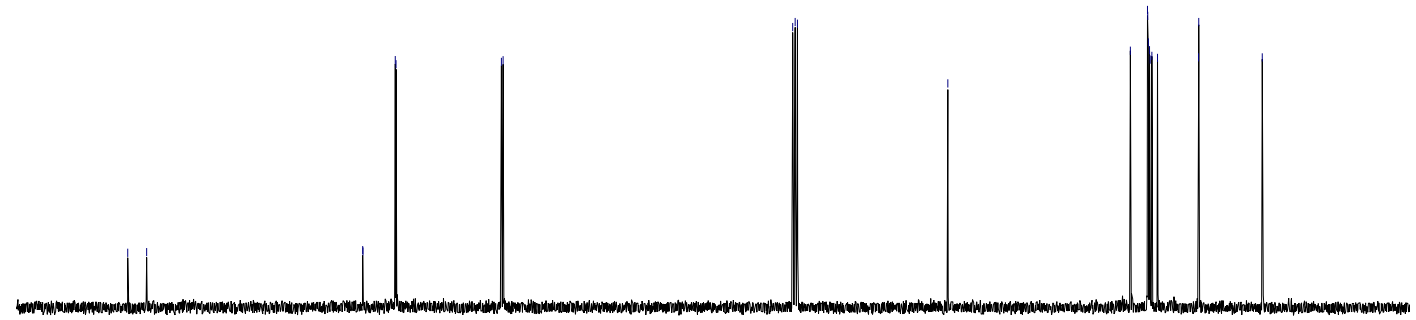

$180 \quad 170$

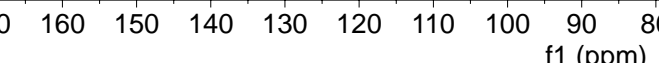
f1 (ppm) 
1-Chloro-4-(dodecylsulfonyl)benzene (4d)

שֶׁ

NNN

৪:

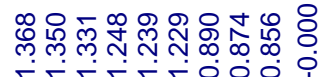

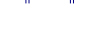
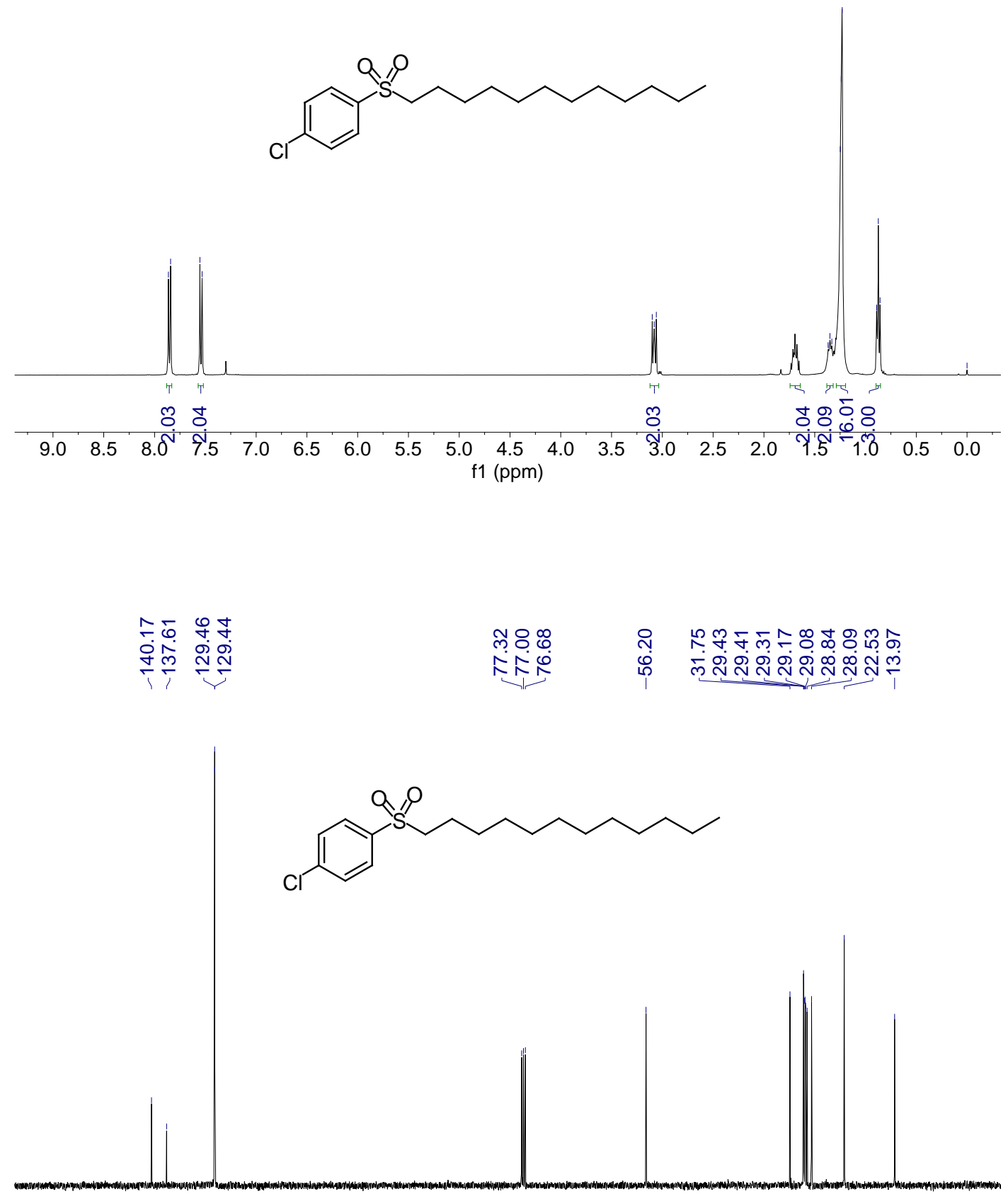


\section{1-Bromo-4-(dodecylsulfonyl)benzene (4e)}

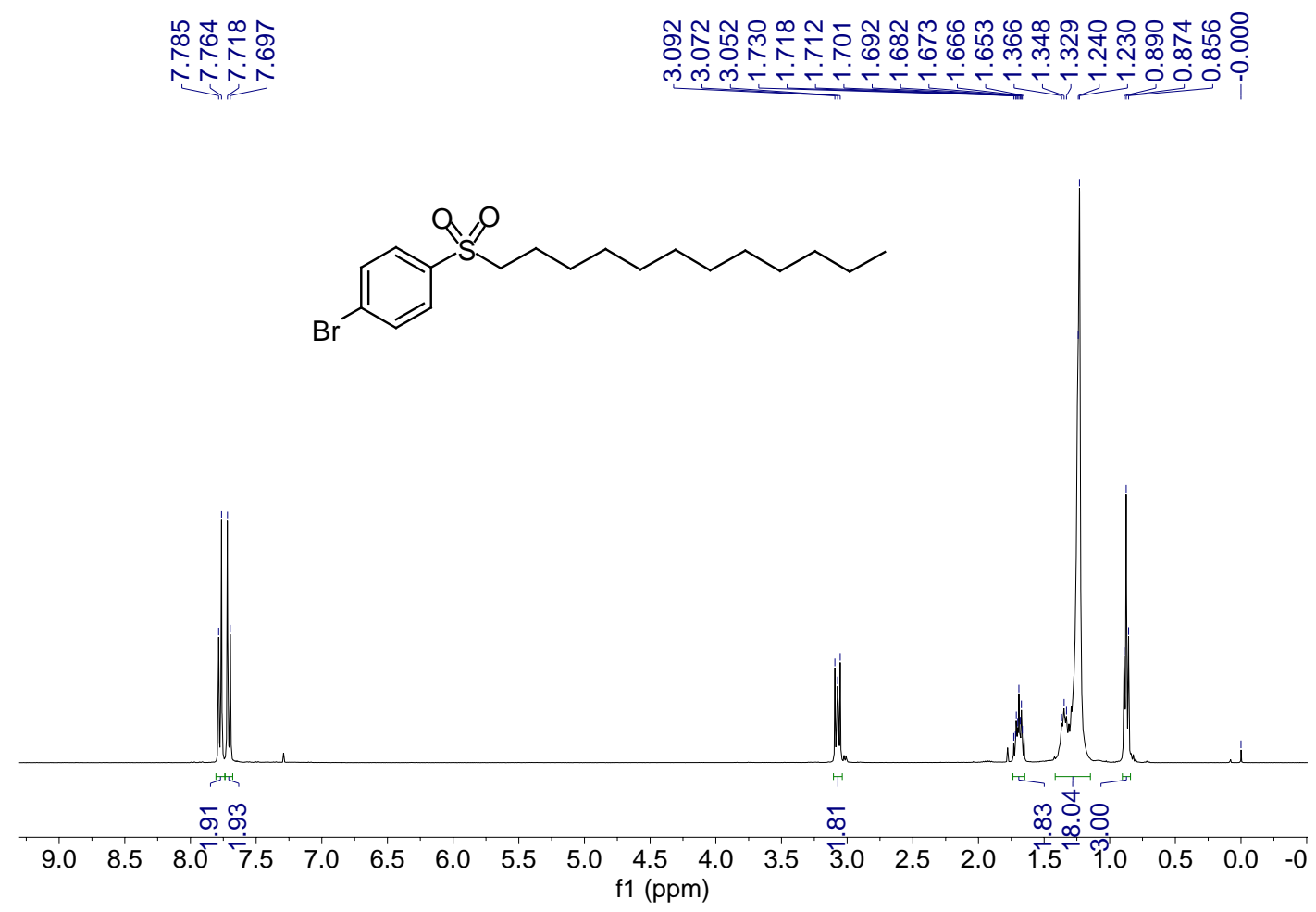

ㄷำ经令

迎

이요

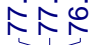

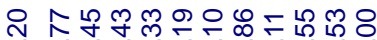

$1 \div$

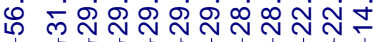

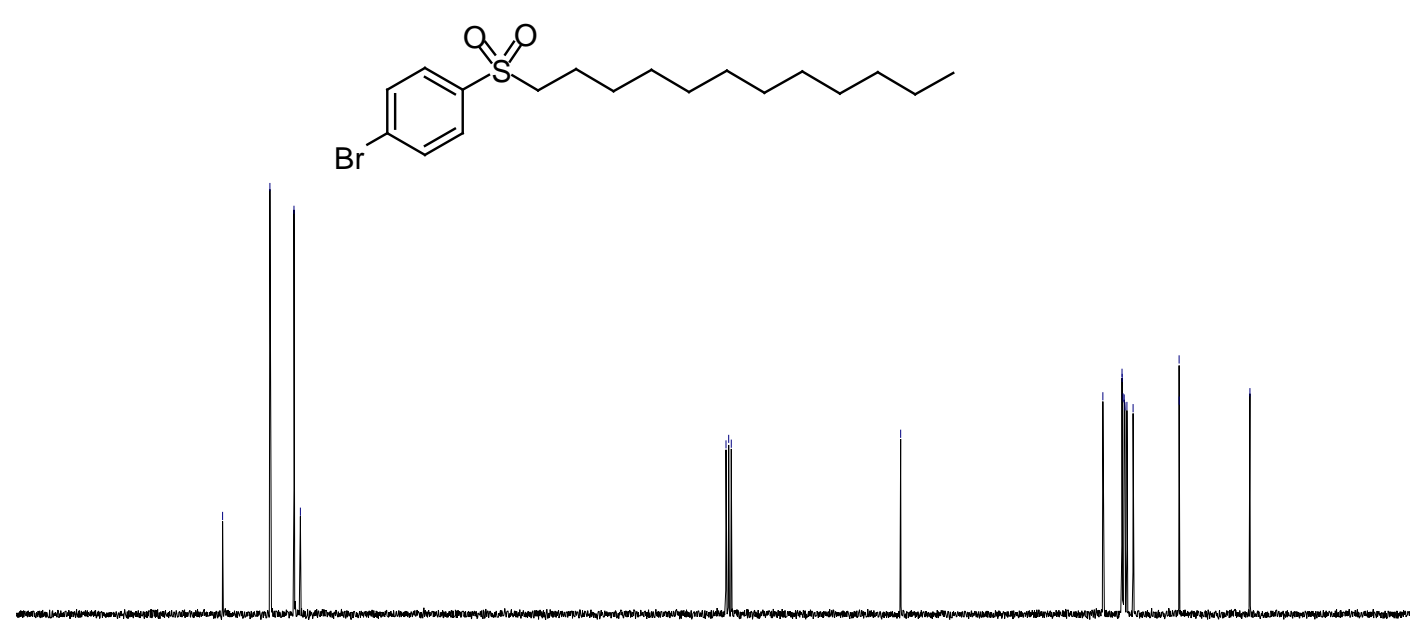

160

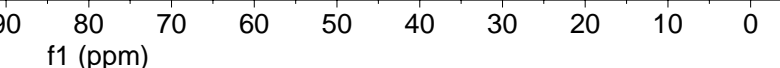




\section{5-((4-Cyanophenyl)sulfonyl)- $N$-phenylpentanamide (4f)}
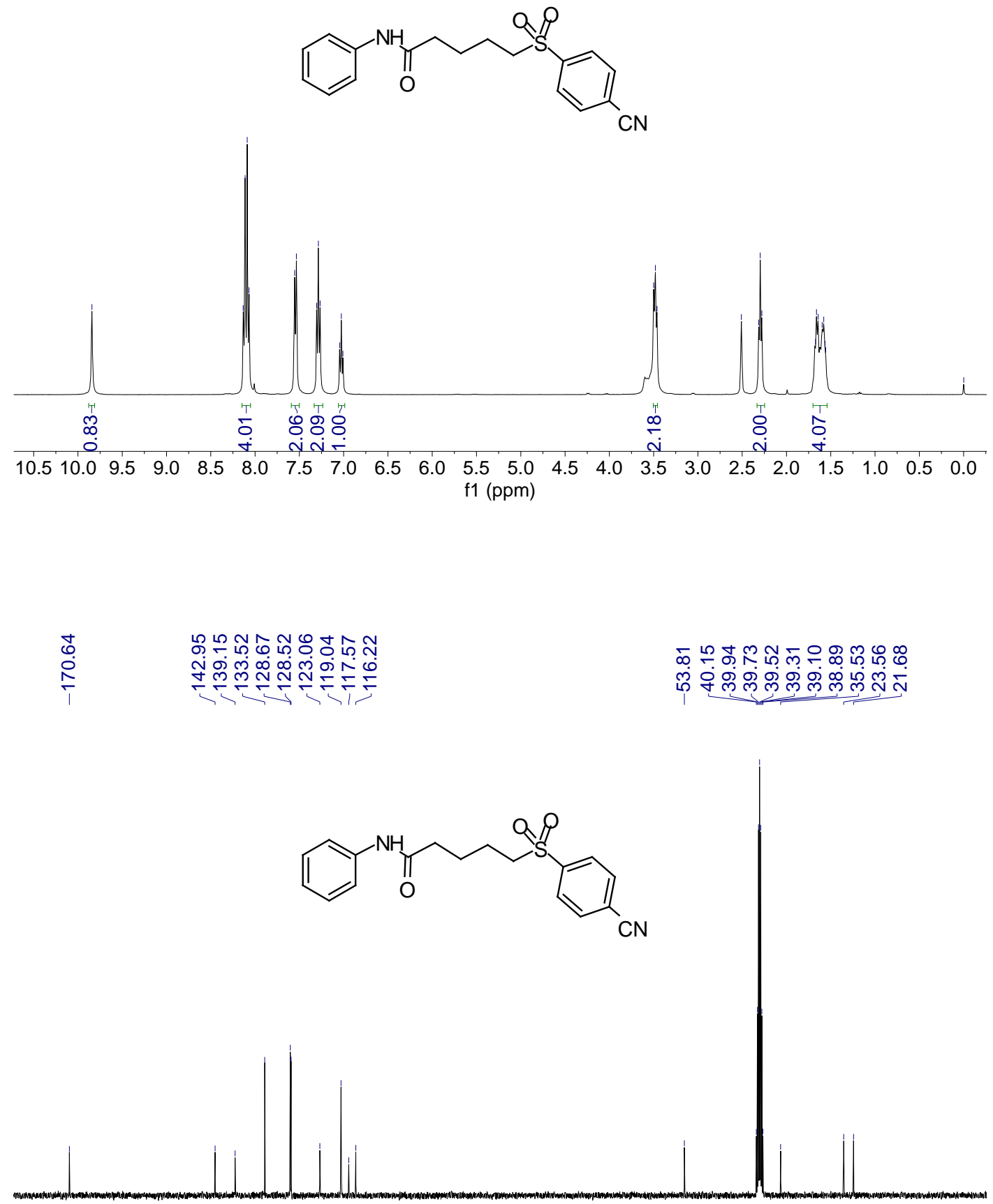

80

$\begin{array}{llllllllllllllllll}70 & 160 & 150 & 140 & 130 & 120 & 110 & 100 & \begin{array}{c}90 \\ \mathrm{f} 1(\mathrm{ppm})\end{array} & 80 & 70 & 60 & 50 & 40 & 30 & 20 & 10 & 0\end{array}$




\section{(Dodecylsulfonyl)cyclopropane (4j)}

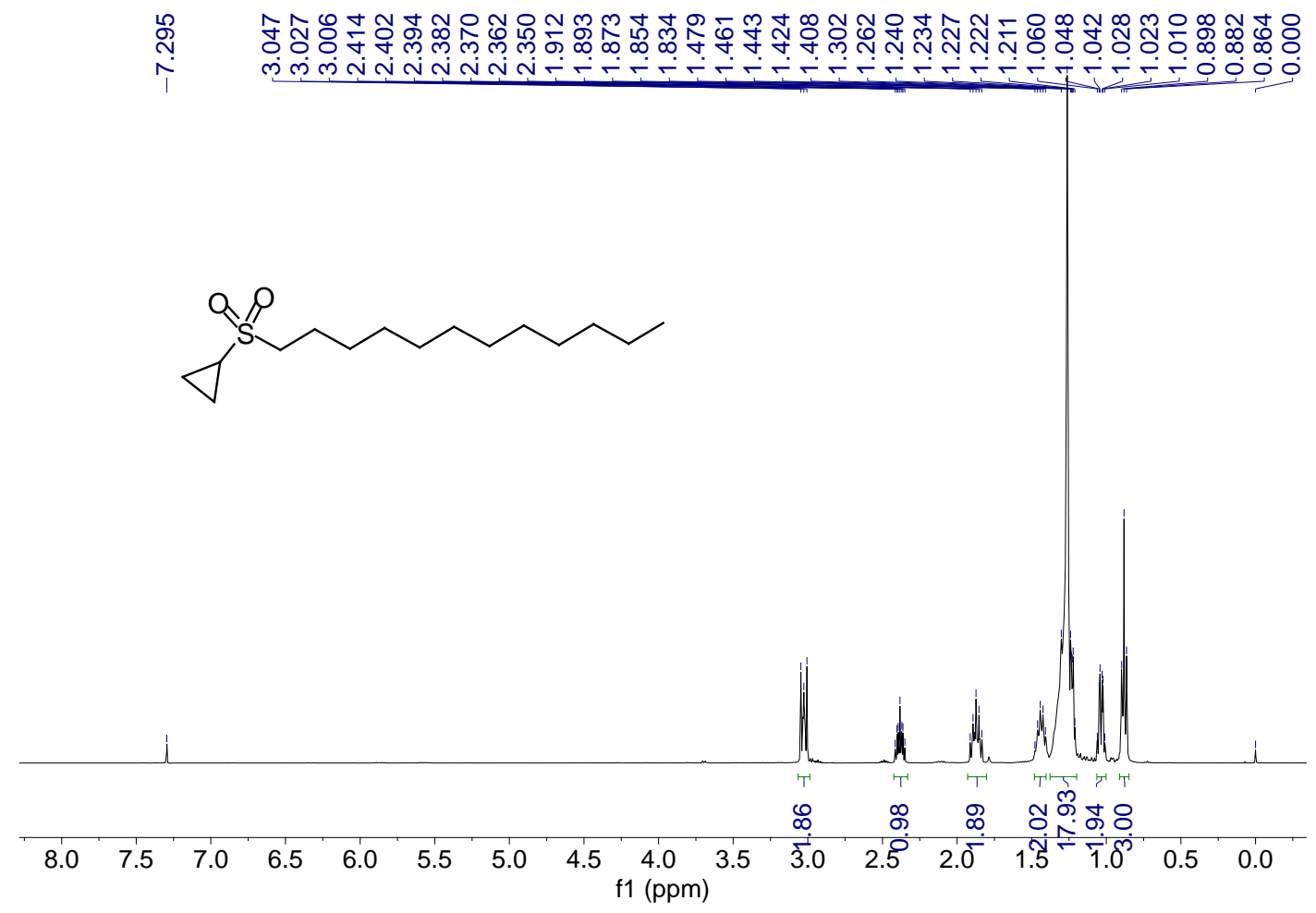

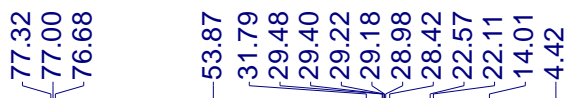
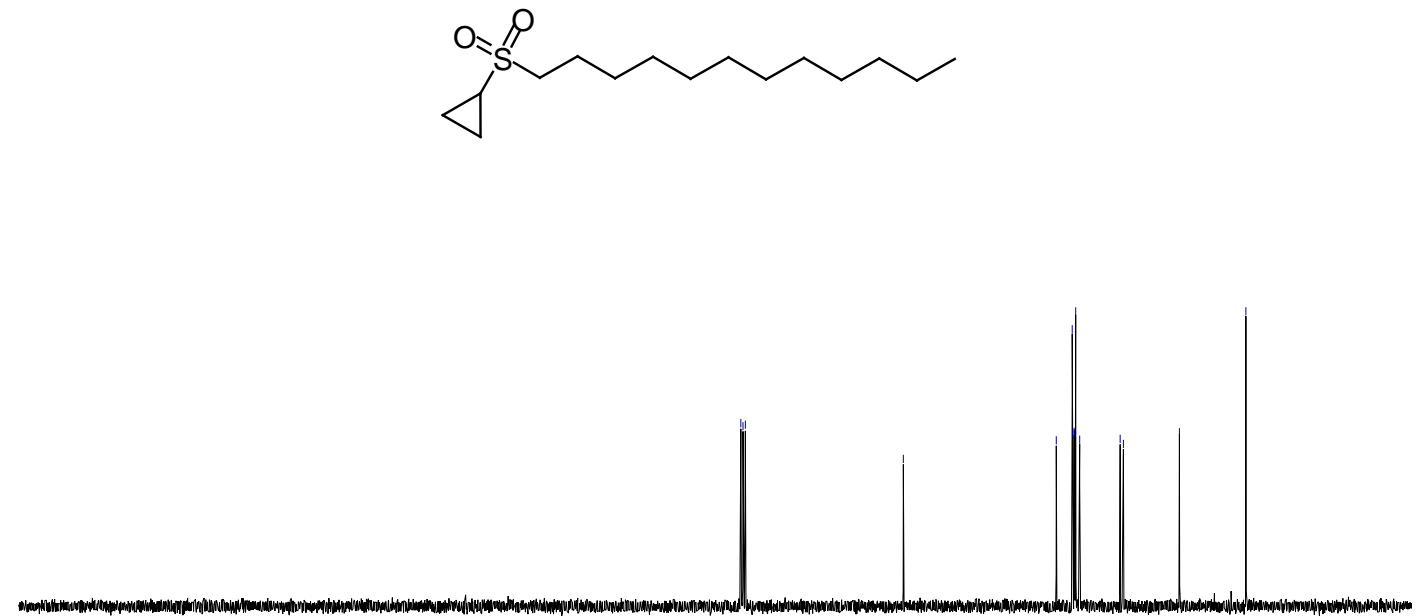

$\begin{array}{llllllllllllllllllll}80 & 170 & 160 & 150 & 140 & 130 & 120 & 110 & 100 & 90 & 80 & 70 & 60 & 50 & 40 & 30 & 20 & 10 & 0 & -10\end{array}$ 

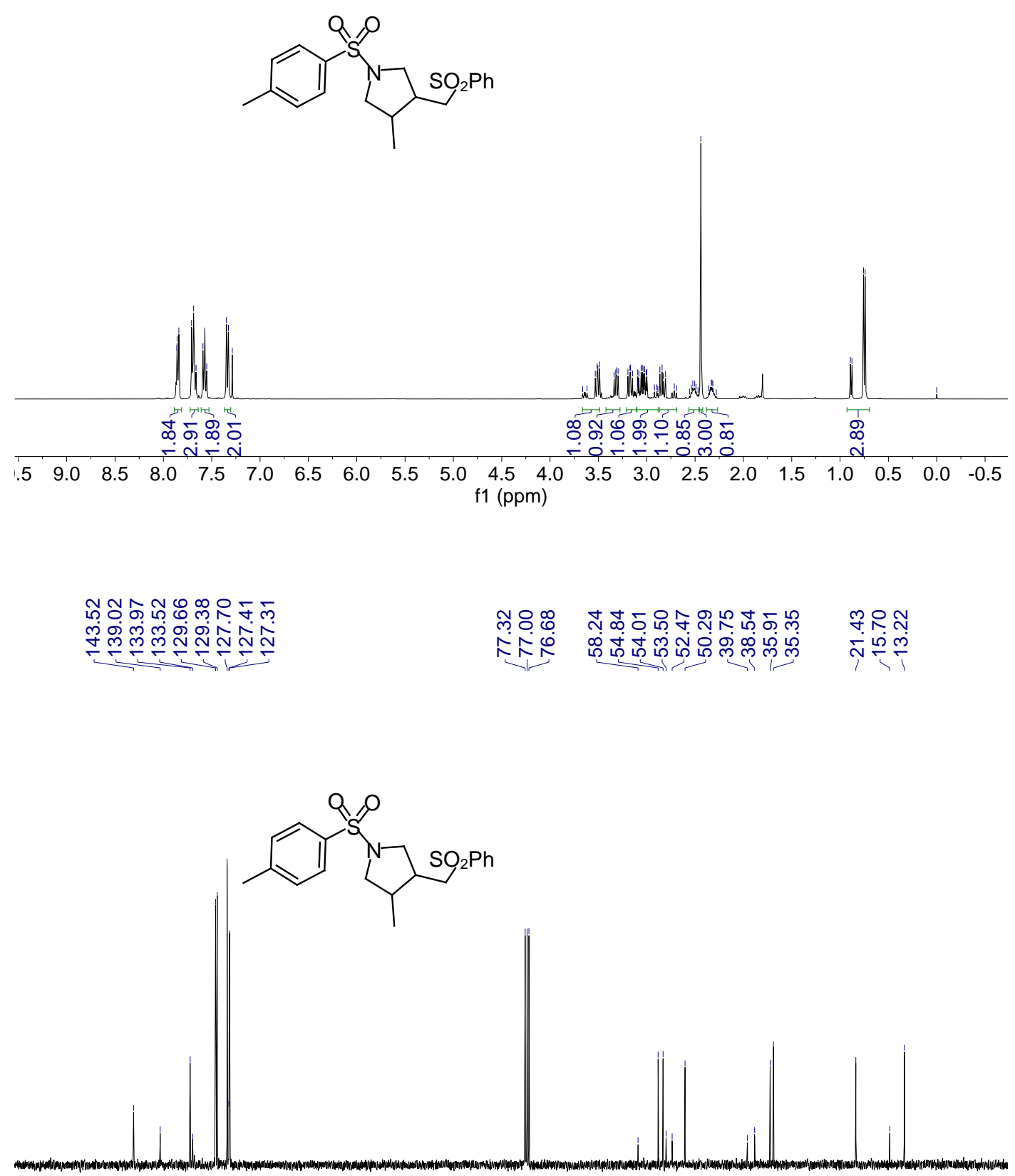


\section{(E)-4-(Phenylsulfonyl)but-2-en-1-ol (8)}

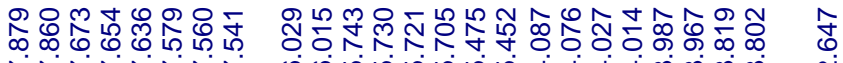

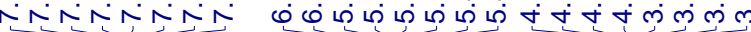

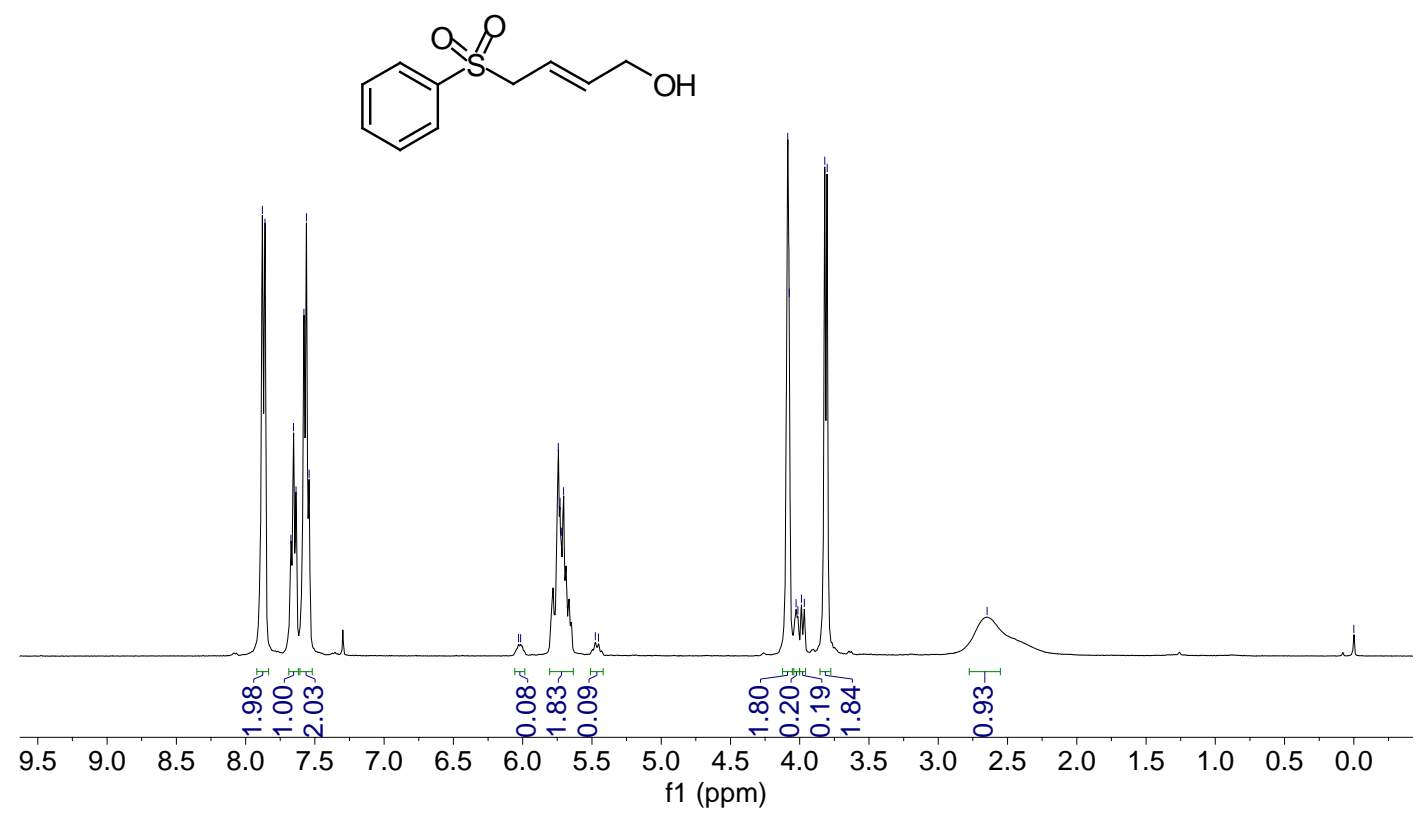

ดำ

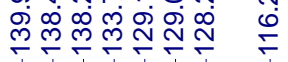<smiles>O=S(=O)(C/C=C/CO)c1ccccc1</smiles>

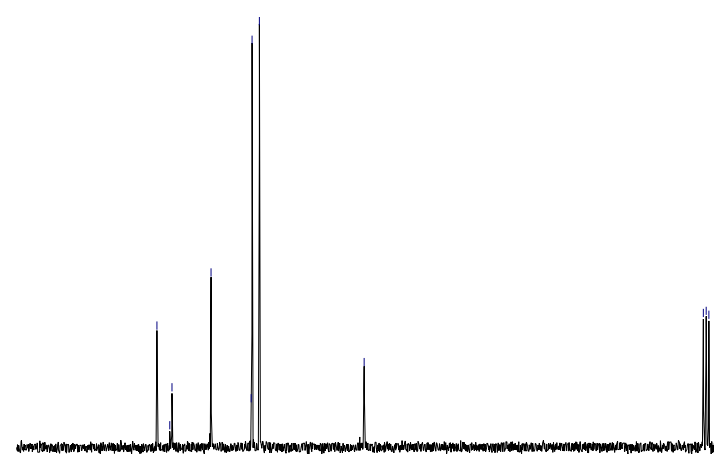

$\begin{array}{llllllllllllllll}150 & 140 & 130 & 120 & 110 & 100 & 90 & \begin{array}{c}80 \\ \mathrm{f} 1(\mathrm{ppm})\end{array} & 60 & 50 & 40 & 30 & 20 & 10 & 0\end{array}$ 
Methyl (1S,2R)-2-((4-chlorophenyl)sulfonyl)cyclopentane-1-carboxylate (9)

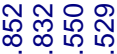

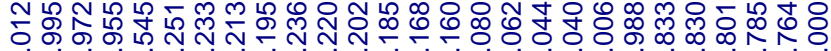

inin

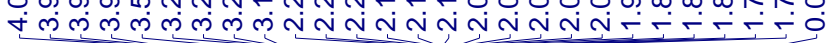

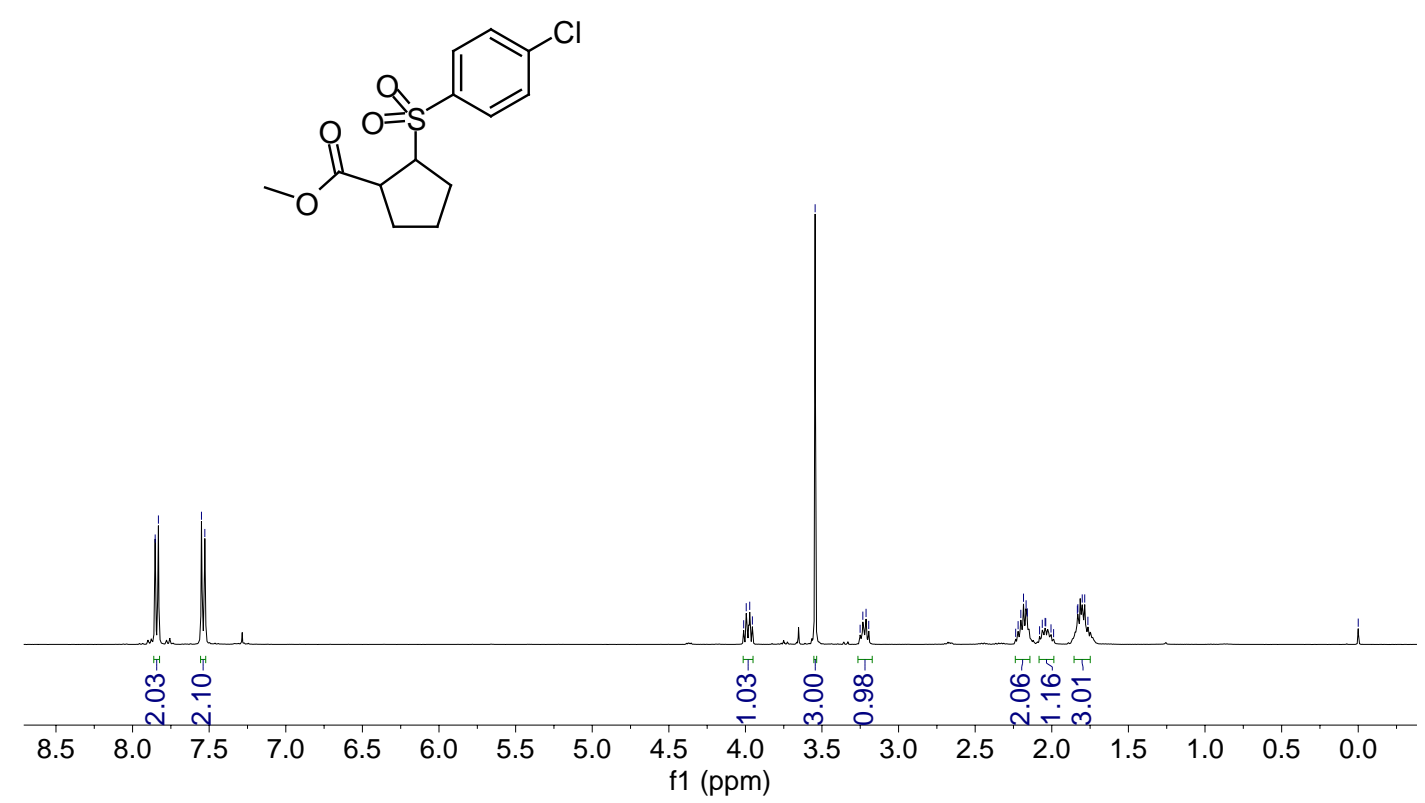

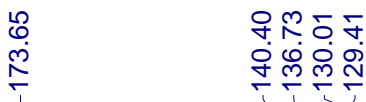

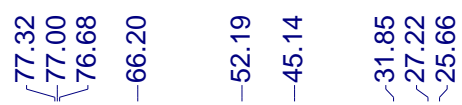<smiles>COC(=O)C1CCCC1S(=O)(=O)c1ccc(Cl)cc1</smiles>

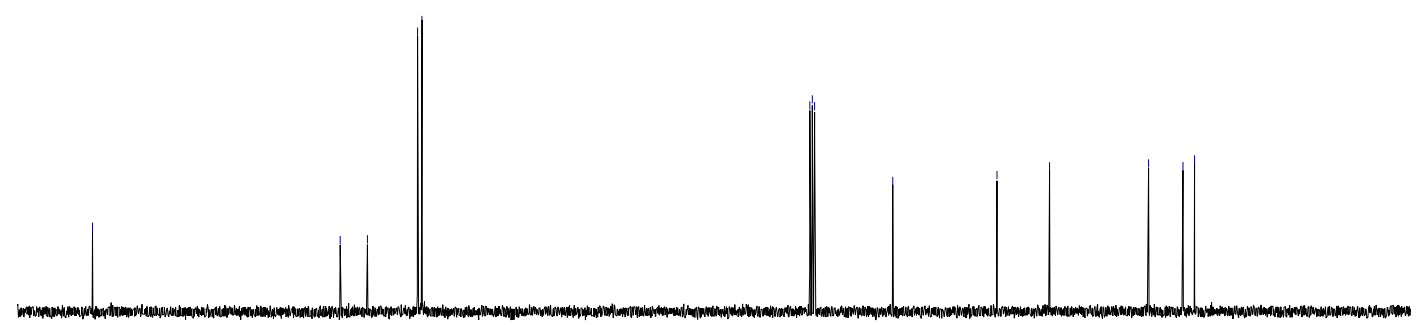

$\begin{array}{lllllllllllllllllll}180 & 170 & 160 & 150 & 140 & 130 & 120 & 110 & 100 & \begin{array}{c}90 \\ \mathrm{f} 1(\mathrm{ppm})\end{array} & 80 & 70 & 60 & 50 & 40 & 30 & 20 & 10 & 0\end{array}$ 


\section{$N$-Phenyl-5-tosylpentanamide (10)}
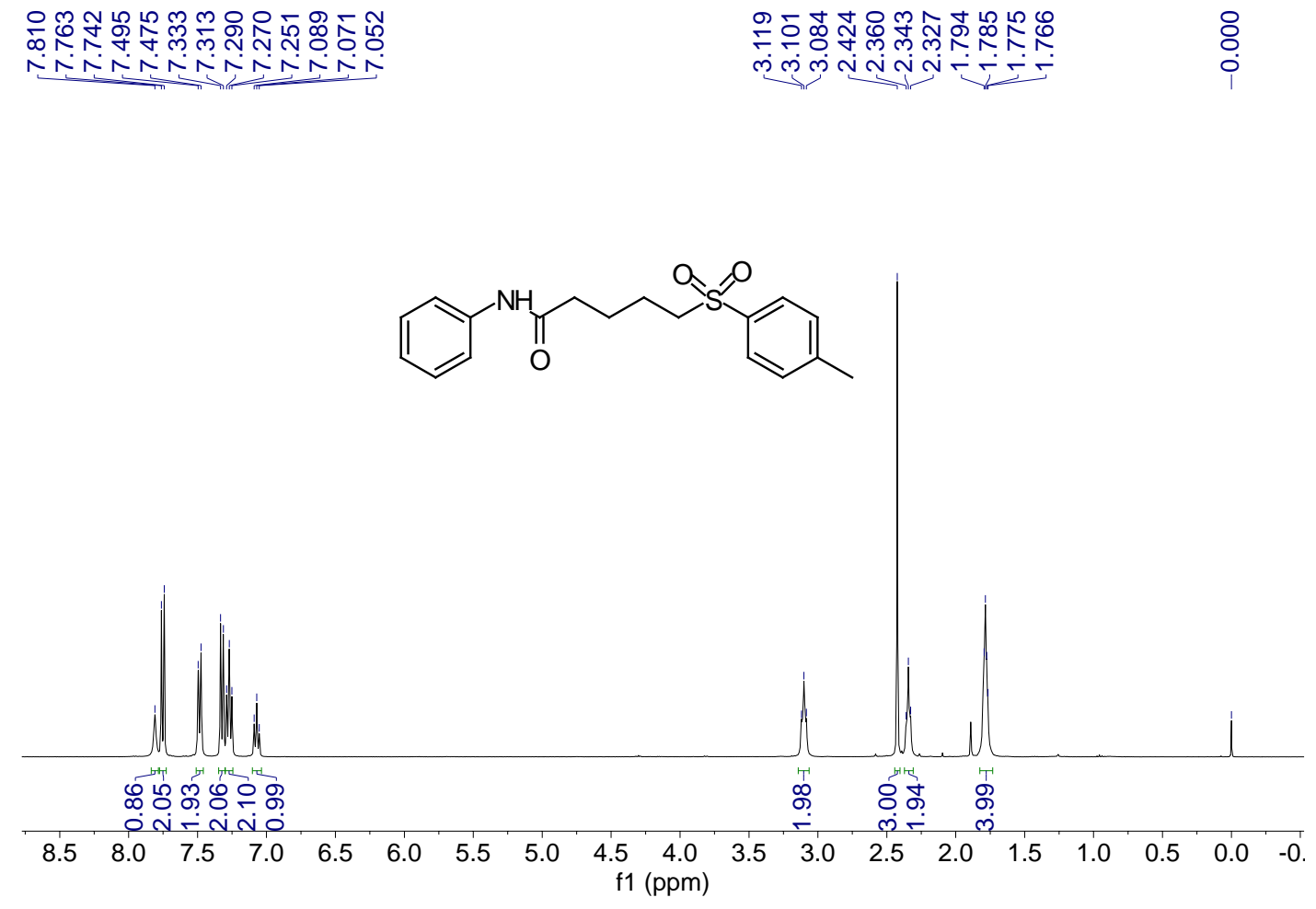

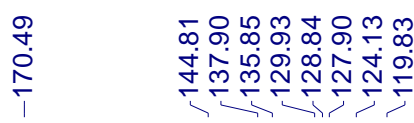

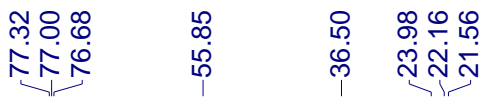<smiles>Cc1ccc(S(=O)(=O)CCCCC(=O)Nc2ccccc2)cc1</smiles>

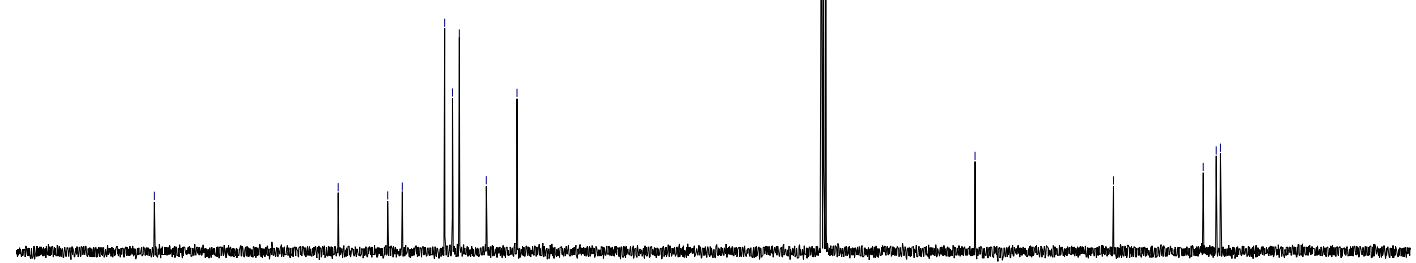

$\begin{array}{lllllllllllllllllll}180 & 170 & 160 & 150 & 140 & 130 & 120 & 110 & \begin{array}{c}100 \\ \mathrm{f} 1(\mathrm{ppm})\end{array} & \begin{array}{c}90 \\ (120\end{array} & 70 & 60 & 50 & 40 & 30 & 20 & 10 & 0\end{array}$ 